\title{
RFQ Designs and Beam-Loss Distributions for IFMIF
}

\author{
R. A. Jameson \\ IFMIF Accelerator Facility Team Leader
}

\section{Hofmann Chart, IFMIF Post-CDR \\ Equipartitioned RFQ}

Written for the IFMIF Project, as a United States contribution to EU Work Package TW5-TTMI-001, Task Deliverable 3

January 2007

International Fusion Materials Irradiation Facility (IFMIF) An Activity of the International Energy Agency (IEA) Implementing Agreement for a Program of Research and Development on Fusion Materials 
This report was prepared as an account of work sponsored by an agency of the United States government. Neither the United States government nor any agency thereof, nor any of their employees, makes any warranty, express or implied, or assumes any legal liability or responsibility for the accuracy, completeness, or usefulness of any information, apparatus, product, or process disclosed, or represents that its use would not infringe privately owned rights. Reference herein to any specific commercial product, process, or service by trade name, trademark, manufacturer, or otherwise, does not necessarily constitute or imply its endorsement, recommendation, or favoring by the United States government or any agency thereof. The views and opinions of authors expressed herein do not necessarily state or reflect those of the United States government or any agency thereof. 


\title{
RFQ Designs and Beam-Loss Distributions for IFMIF
}

\author{
R. A. Jameson \\ IFMIF Accelerator Facility Team Leader
}

Written for the IFMIF Project,

as a United States contribution to EU Work Package TW5-TTMI-001,

Task Deliverable 3

January 2007

International Fusion Materials Irradiation Facility (IFMIF)

An Activity of the International Energy Agency (IEA) Implementing Agreement for a

Program of Research and Development on Fusion Materials

Research sponsored by the Office of Fusion Energy Sciences, U.S. Department of Energy, under Contract DE-AC05-00OR22725 with UT-Battelle, LLC. 


\section{Table of Contents \\ RFQ DESIGNS AND BEAM-LOSS DISTRIBUTIONS FOR IFMIF}

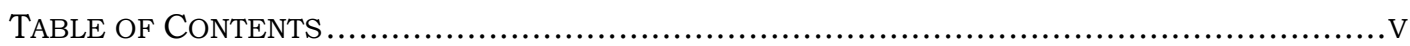

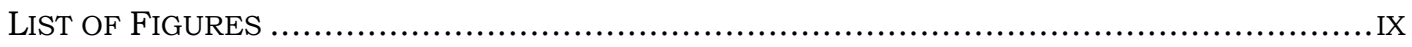

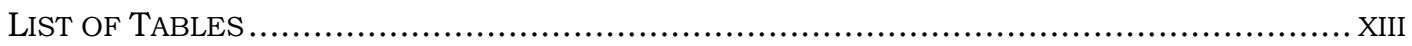

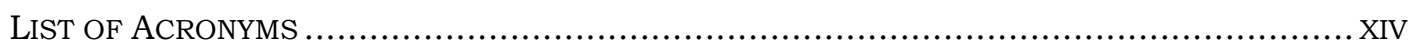

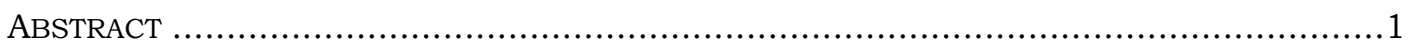

1. INTRODUCTION

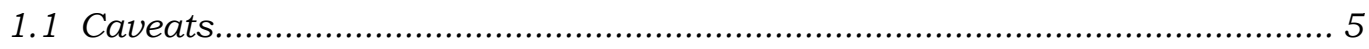

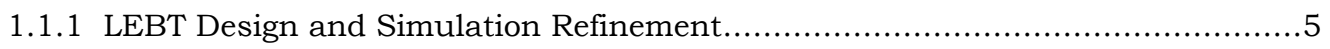

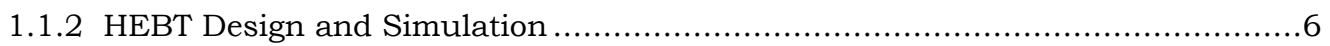

1.1.3 RFQ Design Strategy, Trade-offs, Optimization.........................................6

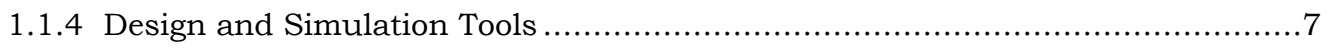

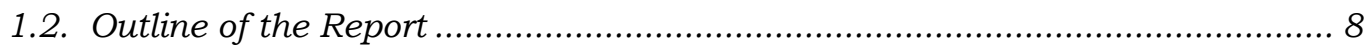

2. PRESENTATION OF THREe IFMIF RFQ DESIGNS .............................................. 11

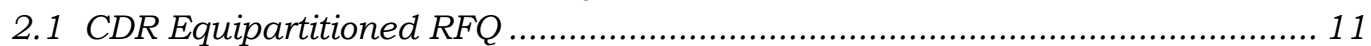

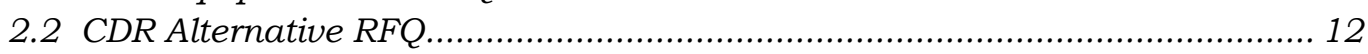

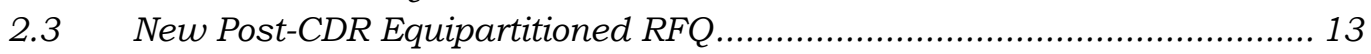

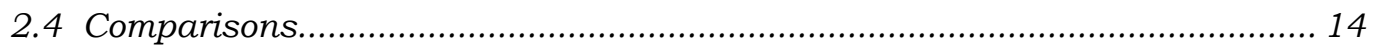

3. BEAM-LOSS DistRIBUTIONS AND ACCELERATED BEAM TRANSMISSION ......................17

3.1 Beam Loss Distribution Comparisons ……............................................... 17

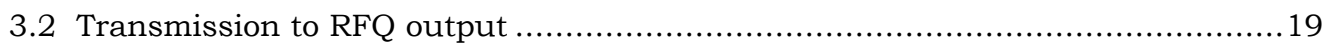

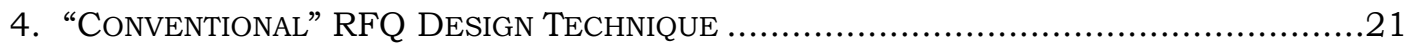

4.1 External Field Quantities as Defined by the RFQ Metal.............................. 21

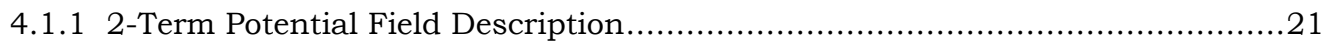

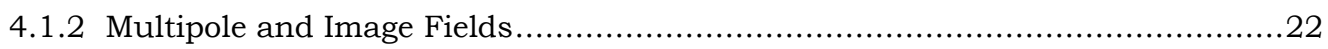

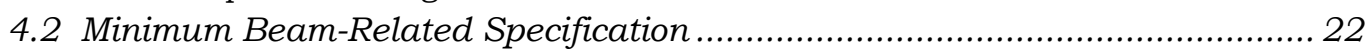

4.2.1 Teplyakov Synchronous Phase Rule .........................................................22

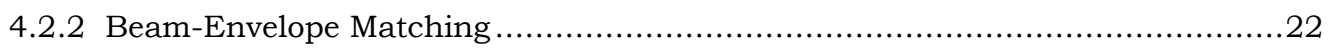

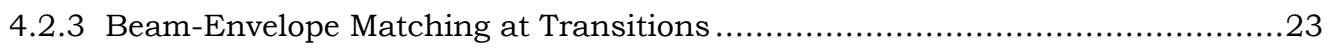

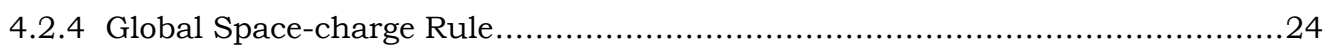

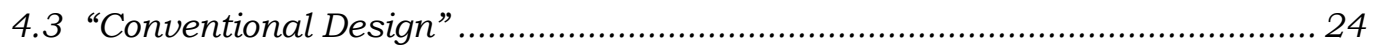

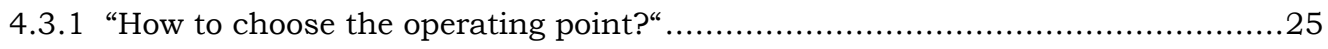

4.3.2 An Optimization Approach to the "Conventional" Design ...............................26

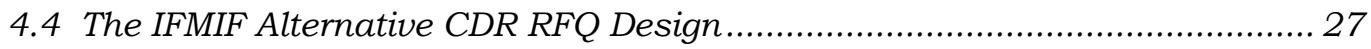

5. BEAM-BASEd LiNEAR ACCELERATOR DESIGN TECHNIQUe .....................................29

5.1 Extension of the "Conventional" Procedure to Achieve Shorter RFQs ............. 30

5.2 Space-charge Physics Relations Between the Accelerator Structure and a

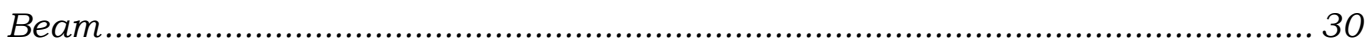

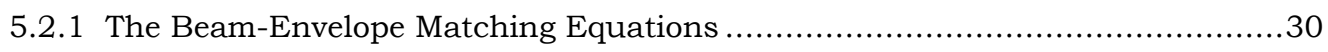

5.2.2 Beam Equilibrium - The Equipartitioned Condition.................................... 31

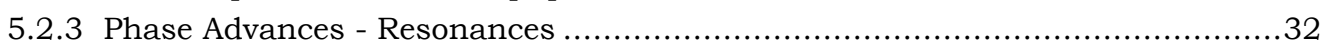

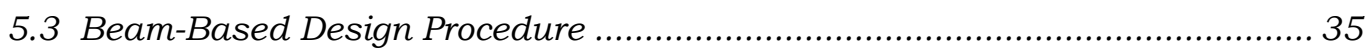

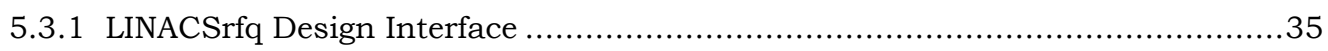

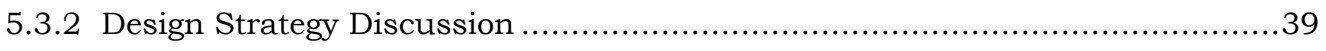

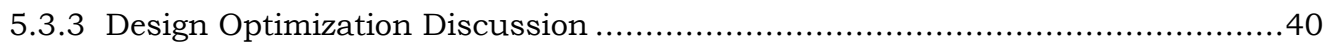


6. Comparisons of the Three RFQs in Terms of Beam-Based Design $\ldots \ldots \ldots \ldots \ldots \ldots . \ldots 41$

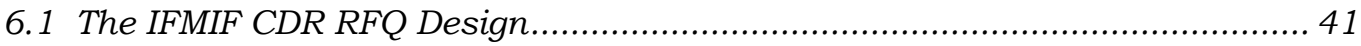

6.2 The IFMIF Post-CDR Equipartitioned RFQ Design..................................... 45

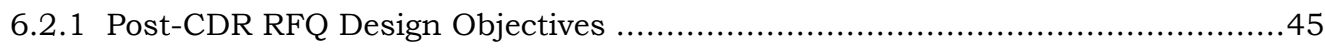

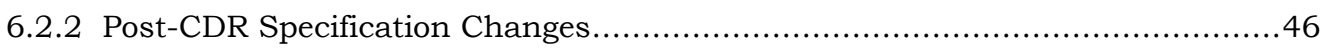

6.2.3 Intermediate Fixed EP Ratio Design ............................................................46

6.2.4 Post-CDR RFQ Varying EP Ratio Design .....................................................48

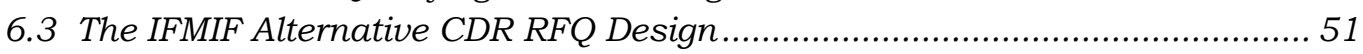

6.4 Beam Size and Emittance Comparison ................................................. 54

6.5 Tune Comparisons ............................................................................... 55

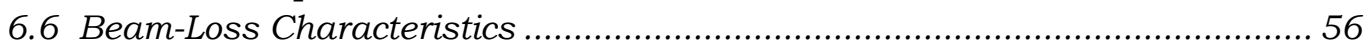

6.6.1 Representative Post-CDR and CDR Equipartitioned-Type RFQ Phase-Space

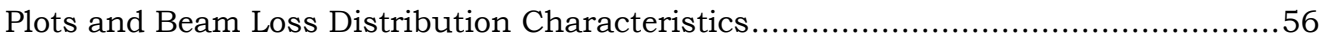

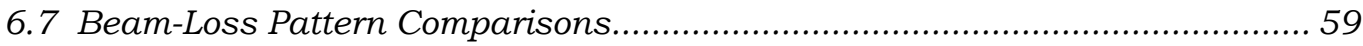

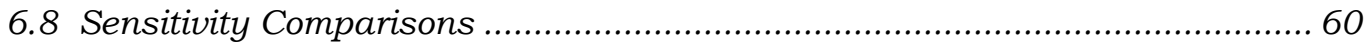

6.8.1 Variation of Input Emittance and Input Current .................................60

6.8.2 Variation of Accelerated Beam with Input Emittance $\alpha$ and $\beta \ldots \ldots \ldots \ldots \ldots \ldots \ldots \ldots . . .62$

6.8.3 Variation of $>1 \mathrm{MeV}$ Losses with Input Emittance $\alpha$ and $\beta$......................64

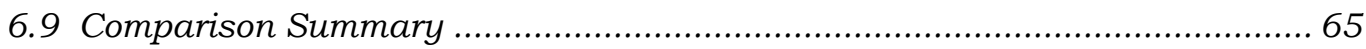

6.9.1 Design Conditions, Strategy and Optimization .....................................65

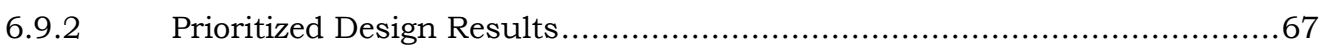

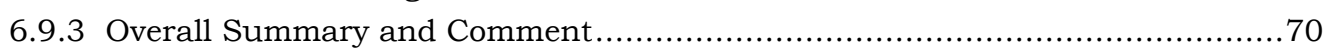

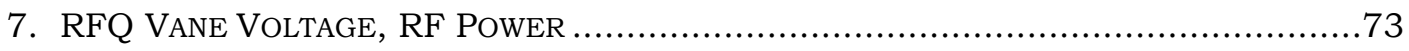

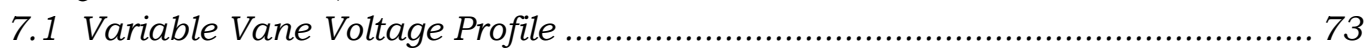

7.1.1 The Russian IFMIF CDA Preliminary RFQ Proposal ....................................

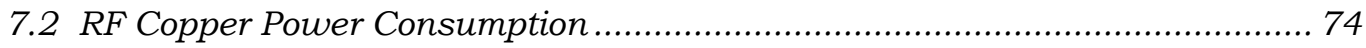

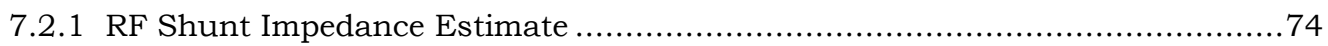

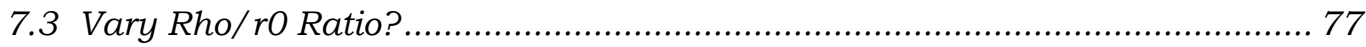

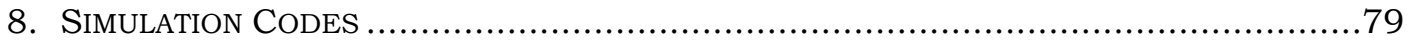

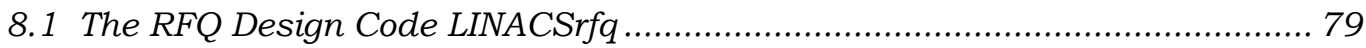

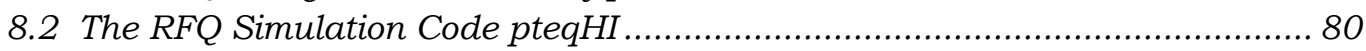

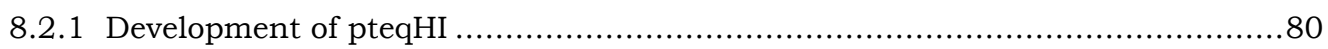

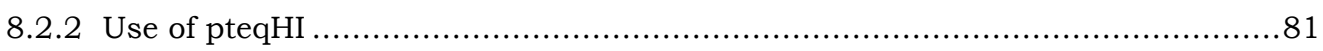

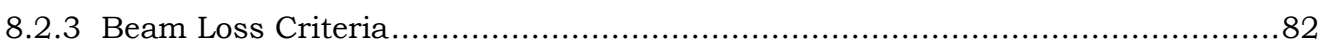

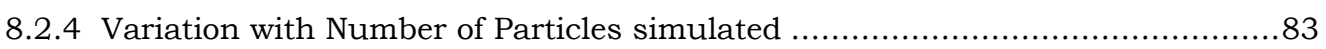

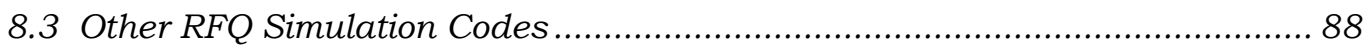

9. ION-SOURCE/LEBT INPUT BEAM MOdELING ............................................. 91

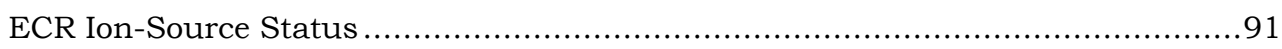

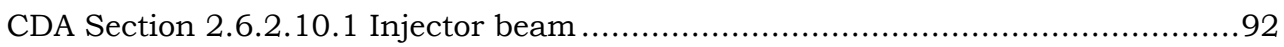

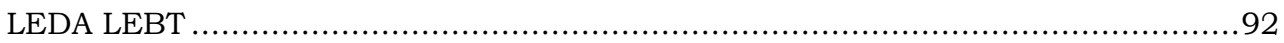

CDA LEBT - CDA Section 2.6.2.2, Fig. 2.6.2-3 …........................................ 92

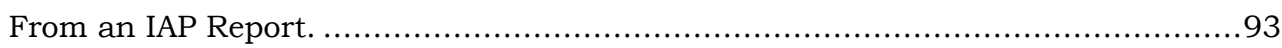

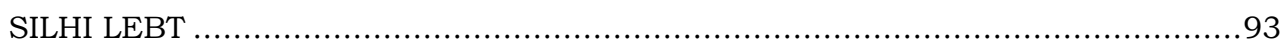

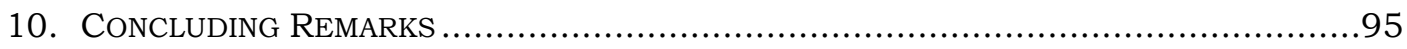

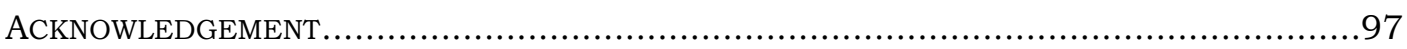

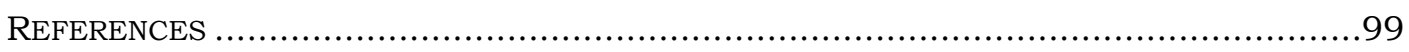


APPENDIX

Post-CDR RFQ LINACS Summary Table

Post-CDR RFQ PteqHI tapeinput

Post-CDR RFQ Cell Table

CDR RFQ PteqHI tapeinput

CDR RFQ Cell Table

AltCDR RFQ PteqHI tapeinput

AltCDR RFQ Cell Table 


\section{List of Figures}

Fig. 2.1-1. Vane parameters for the $140 \mathrm{~mA}$ CDR equipartitioned RFQ. Aperture (a) and trms $=$ transverse $\mathrm{rms}$ beam radius are in $\mathrm{cm} .(\mathrm{m})$ is the vane modulation, $\mathrm{V}$ is the vane voltage, $\mathrm{B}$ is the transverse focusing strength. Phi is the synchronous phase (phis). Rho $/ \mathrm{rO}=0.75$. Input current $=140 \mathrm{~mA}$, input energy $=0.100 \mathrm{MeV}$, input transverse normalized rms emittance $=0.20$ mm.mrad.

Fig. 2.2-1. Vane parameters for the $130 \mathrm{~mA} C D R$ Alternative RFQ. Input current $=$ $130 \mathrm{~mA}$, input energy $=0.095 \mathrm{MeV}$, input transverse normalized rms emittance $=0.25 \mathrm{~mm} . \mathrm{mrad}$.

Fig. 2.2-2. The Rho/rO ratio is varied, to give lower peak surface field (lower KP factor) in the downstream part of the RFQ. Epeak (KP) is the KP factor, ckappa is the ratio between the peak field on the vane surface and the vane-tip field.....12

Fig. 2.3-1. Vane parameters for the $130 \mathrm{~mA}$ New Post-CDR RFQ. Input current $=$ $130 \mathrm{~mA}$, input energy $=0.095 \mathrm{MeV}$, input transverse normalized rms emittance $=0.25 \mathrm{~mm} . \mathrm{mrad}$.

Fig. 2.4-1,2. Comparison of minimum aperture, a (nearly the same for the three RFQs), and average aperture, rO.

Fig.2.4-3,4. Comparison of vane voltage and accelerating factor ez...................... 14

Fig. 2.4-4,5. Comparison of accelerating efficiency A and synchronous phase phis...15

Fig. 2.4-7,8. Comparison of vane modulation $\mathrm{m}$ and focusing factor B. .................. 15

Fig. 2.4-9,10. Comparison of Copper power per cell and integrated copper power.....15

Fig. 3.1-1. \% of all lost particles vs. energy where lost, $0.0-0.3 \mathrm{MeV}$. ....................18

Fig. 3.1-3. \% of all lost particles vs. energy where lost, $0.0-5.05 \mathrm{MeV}$, expanded

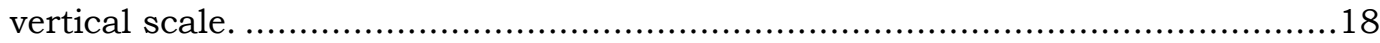

Fig. 3.2-1. \% Accelerated particles from Source Emittance initial distribution vs cell

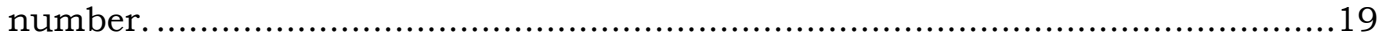

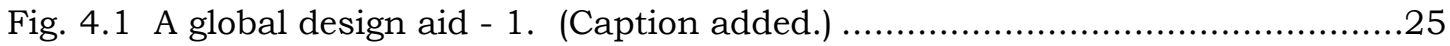

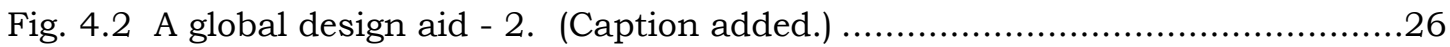

Fig. 5.2.3-1. Hofmann Chart for longitudinal-to-transverse emittance ratio eln/etn = 2.0; thus $\mathrm{kz} / \mathrm{kx}=0.5$ is the equipartitioned condition for this chart..................33

Fig. 6.1-1. Equipartitioning ratio, and corresponding beam size, emittance and tune ratios, Eqs. (7) and (8) for the IFMIF CDR RFQ using the 2-term potential. (Ignore the file ID material after the commas.)

Fig. 6.1-2. Hofmann Chart for eln/etn=2.0, showing the trajectory for the IFMIF CDR RFQ from the EOS to the output, using the 2-term potential.

Fig. 6.1-3. Longitudinal and transverse normalized rms emittances through the IFMIF CDR RFQ.

Fig. 6.1-4. Equipartitioning ratio, and corresponding beam size, emittance and tune ratios, Eqs. (7) and (8) for the IFMIF CDR RFQ using pteqHI including multipole and image-charge effects.

Fig. 6.1-5. Hofmann Chart for eln/etn=2.0, showing the IFMIF CDR RFQ trajectory for the shaper and from the EOS to the output, using pteqHI including multipole and image-charge effects.

Fig. 6.1-6. Transverse and longitudinal rms normalized emittances for the IFMIF CDR RFQ, using pteqHI including multipole and image-charge effects. 
Fig. 6.2-1. Equipartitioning ratio, and corresponding beam size, emittance and tune ratios, Eqs. (7) and (8) for the intermediate RFQ using pteqHI including multipole and image-charge effects.

Fig. 6.2-2. Hofmann Chart for eln/etn=1.4, showing the intermediate RFQ trajectory for the shaper and from the EOS to the output, using pteqHI including multipole and image-charge effects.

Fig. 6.2-3. Transverse and longitudinal rms normalized emittances for the intermediate RFQ, using pteqHI including multipole and image-charge effects...48

Fig. 6.2-4. Equipartitioning ratio, and corresponding beam size, emittance and tune ratios, Eqs. (7) and (8) for the Post-CDR equipartitioned RFQ using pteqHI including multipole and image-charge effects.

Fig. 6.2-5. Composite Hofmann Chart for eln/etn=1.4 (underlying blue-toned shadows) and eln/etn $=2$ (overlying magenta toned shadows). The Post-CDR equipartitioned RFQ trajectories for the shaper and from the EOS to the output are shown, using pteqHI including multipole and image-charge effects.

Fig. 6.2-6. Transverse and longitudinal rms normalized emittances for the Post-CDR equipartitioned RFQ, using pteqHI including multipole and image-charge effects.

Fig. 6.3-1. Equipartitioning ratio, and corresponding beam size, emittance and tune ratios, Eqs. (7) and (8) for the IFMIF Alternative CDR RFQ using pteqHI including multipole and image-charge effects.

Fig.6.3-2. Hofmann chart for the AltCDR RFQ. Chart is for emittance ratio of 1.4. Beam reached equipartitioned equilibrium briefly at emittance ratio of 1.6 (Fig.6.3-1). (On a chart with 1.6 emittance ratio, the resonance at $\mathrm{kz} / \mathrm{kx}=0.5$ would be weakened, and the resonance to the left of $\mathrm{kz} / \mathrm{kx}=1$ would be stronger).

Fig. 6.3-3. Transverse and longitudinal rms normalized emittances for the IFMIF Alternative CDR RFQ, using pteqHI including multipole and image-charge effects.

Fig. 6.3-4a. AltCDR RFQ z-z' phase space at the end of the RFQ; 4b - expanded scale

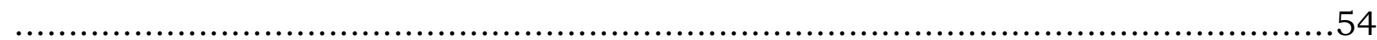

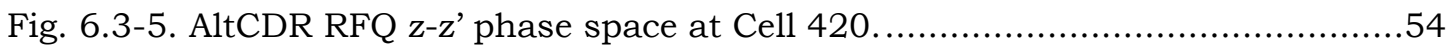

Fig. 6.4-1. Rms beam radius (trms) and length (zrms), cm.................................54

Fig. 6.4-2. Rms normalized transverse (etn) and longitudinal (eln) emittances, cm.rad. The AltCDR and CDR/PostCDR eln are not qualitatively similar, as discussed above. The transverse input distribution is an ideal waterbag, $100 \mathrm{~K}$ particles, etn $=0.25 \mathrm{~mm}$. mrad for each RFQ; an immediate redistribution in the transverse emittance occurs as the distribution adapts to the RFQ.

Fig. 6.5-1. Zero-current phase advances/transverse focusing period, degrees...........55

Fig. 6.5-2. Zero-current phase advances $\sigma_{0}$, and depressed tunes $\sigma / \sigma_{0}$, for the three

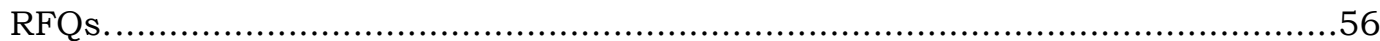

Fig. 6.5-3 Side-by-side comparison of the Hofmann Charts for the three RFQs........56

Plots are at the middle of the last cell. Black: $1 \mathrm{M}$ particle ideal waterbag; Red: $\sim 1 \mathrm{M}$ particle ion source distribution rms matched to the RFQ. ............................56

Fig. 6.6-9a,b. RFQ, with ion source input. Particle energy of all losses at each cell. There is a spectrum of lost particle energies at each cell, from $\sim 0.05 *$ (synchronous energy) to $\sim$ (synchronous energy).

Fig. 6.7-1. Fig. 3.1-3 repeated; beam loss for $\sim 1 \mathrm{M}$ particle ion source emittance distribution, expanded scale. 
Fig. 6.7-2. \% accelerated particles vs. cell number, Source Emittance initial distribution.

Figs. 6.8-1-a,b,c. Variation with input

Figs. 6.8-2-a,b,c. Variation with

Fig.6.8-3. CDR RFQ - \% Accelerated beam sensitivity to input emittance $\alpha$ and $\beta$. The design $\alpha$ and $\beta$ are as found for the original CDR RFQ evaluated without multipole or image fields. At the design $\alpha=1.7008, \beta=12.7828$, $\{$ AccBeam, \%Loss $>1 \mathrm{MeV}\}$ is $\{89.6,0.034\}$. At $\alpha=1.4, \beta=11.5,\{89.9,0.030\}$; at $\alpha=1.4, \beta=$ 12.75, \{89.6,0.014\}; (Fig. 6.8-6).

Fig.6.8-4. AltCDR RFQ - Accelerated beam sensitivity to input emittance $\alpha$ and $\beta$. At the design $\alpha=2.362, \beta=14.2,\{\%$ AccBeam, \%Loss $>1 \mathrm{MeV}\}$ is $\{90.0,0.066\}$. At $\alpha=2.25, \beta=12.75,\{91.3,0.080\}$; at $\alpha=2.47, \beta=15$., \{90.7,0.058\}; (Fig. 6.8-7).

Fig.6.8-5. Post-CDR RFQ - Accelerated beam sensitivity to input emittance $\alpha$ and $\beta$. The design $\alpha=1.2, \beta=11$, as found during the optimization; $\{\%$ AccBeam, \%Loss $>1 \mathrm{MeV}\}$ is $\{98.0,0.101\}$. At $\alpha=1.3, \beta=10.9,\{97.0,0.074\}$; at $\alpha=1.1, \beta=$ 10.1, \{96.9,0.076\}; (Fig. 6.8-8).

Fig.6.8-6. CDR RFQ - >1MeV-losses sensitivity to input emittance $\alpha$ and $\beta$. At the design $\alpha=1.7008, \beta=12.7828$, $\{\%$ AccBeam, \%Loss $>1 \mathrm{MeV}\}$ is $\{89.6,0.034\}$. At $\alpha$ $=1.4, \mathrm{~b}=11.5,\{89.9,0.030\}$; at $\alpha=1.4, \beta=12.75$, \{89.6,0.014\}; (Fig. 6.8-3) .....64

Fig.6.8-7. AltCDR RFQ - >1MeV-losses sensitivity to input emittance $\alpha$ and $\beta$. At the design $\alpha=2.362, \beta=14.2$, $\{\%$ AccBeam, \%Loss $>1 \mathrm{MeV}\}$ is $\{90.0,0.066\}$. At $\alpha=$ 2.25, $\beta=12.75,\{91.3,0.080\}$; at $\alpha=2.47, \beta=15$., $\{90.7,0.058\}$; (Fig. 6.8-4)......64

Fig.6.8-8. Post-CDR RFQ - >1MeV-losses sensitivity to input emittance $\alpha$ and $\beta$. At the design $\alpha=1.2, \beta=11$, $\{\%$ AccBeam, \%Loss $>1 \mathrm{MeV}\}$ is $\{98.0,0.101\}$. At $\alpha=$ $1.3, \beta=10.9$, $\{97.0,0.074\}$; at $\alpha=1.1, \beta=10.1,\{96.9,0.076\}$; (Fig. 6.8-5).

Fig. 6.9-1. Sensitivity of accelerated beam fraction and $\%>1 \mathrm{MeV}-1$ losses to input emittance. Repeat of fig. 6.8-1; left-to-right CDR, AltCDR, Post-CDR.

Fig. 6.9-2. Sensitivity of accelerated beam fraction and $\%>1 \mathrm{MeV}-1$ osses to input current. Repeat of fig. 6.8-2; left-to-right CDR, AltCDR, Post-CDR.

Fig. 6.9-3. Loss patterns for $\sim 1 \mathrm{M}$ particle Source emittance input distribution, repeat of Fig. 3.1-3. It is necessary to use a simulation code with canonical (time) coordinates for accurate loss patterns. Left-to-right CDR, AltCDR, PostCDR.

Fig. 6.9-4. \% losses $>1 \mathrm{MeV}$, repeat of figs. 6.8-6,7,8. Left-to-right CDR, AltCDR, Post-CDR. Produced from $100 \mathrm{~K}$ particle simulations.

Fig. 6.9-5. Accelerated particles from $100 \mathrm{~K}$ waterbag input distribution, repeat of Figs. 6.8-6,7,8. Left-to-right CDR, AltCDR, Post-CDR. (Different scale for PostCDR).

Fig. 6.9.3-1. Repeat of Figs. 6.4-1 and 6.4-2. Rms beam size and emittance comparisons.

Fig. 7.1.1-1. Parameter table for 0.1-3.0 MeV and 3.0-8.0 MeV RFQs for IFMIF proposed by IHEP.

Fig. 7.2.1-1. RFQUIK estimate for rf shunt impedance Rs along the IFMIF CDR RFQ.

Fig. 7.2.1-2. Rf shunt impedance data from operating RFQs..............................76

Fig. 8.2-1. Quadrupolar function with $+-10^{\circ}$ vanetip opening angle, computed at each step for $\mathrm{x}$ - and $\mathrm{y}$ - location of actual vane tips. 
Fig. 8.2.2. Left - \% accelerated particles, $100 \mathrm{~K}$ waterbag initial distribution; Right -

$\%$ accelerated particles, Source Emittance initial distribution.

Fig. 8.2-3. Post-CDR RFQ. \% of all lost particles vs. energy where lost, $0.0-0.3$ $\mathrm{MeV}$.

Fig. 8.2-4a,b. Post-CDR RFQ. \% of all lost particles vs. energy where lost, expanded vertical scales. For $5 \mathrm{~K}, 10 \mathrm{~K}, 100 \mathrm{~K}, 1 \mathrm{M}$ particles, loss of 1 particle is $0.02 \%$, $0.01 \%, 0.001 \%, 0.0001 \%$. (400 bins, each $5.05 \mathrm{MeV} / 400=0.012625 \mathrm{MeV}) \ldots \ldots . .86$

Fig. 8.2-5. Post-CDR RFQ. \% of all lost particles vs. position ( $z$, meters) where lost.

Fig. 9.1 Raw Data Characteristics of the ECR Ion-Source Emittance Distribution (see figures, Section 6.6) Right figure has expanded vertical scale. .................91

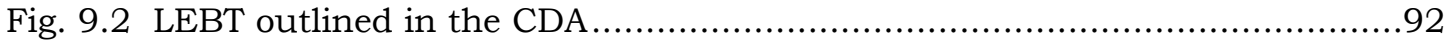

Fig. 9.3 A representative LEBT outlined by IAP .............................................. 93

Fig. 9.4 A LEBT considered by Saclay includes a collimator..................................93 


\section{List of Tables}

Table 1-1. Top-level specification for the IFMIF Accelerator Facility ….......................

Table 1-2. Top-level specification for the IFMIF RFQ.......................................4

Table 6.9-1 Summary of design conditions, strategy and optimization (a.-e.) and prioritized results (1.-5.) at the design match ..............................................65

Table 8.2-1. CDR RFQ - Variation of transmission with number of particles simulated.

Table 8.2-2. AltCDR RFQ - Variation of transmission with number of particles simulated.

Table 8.2-3. PostCDR RFQ - Variation of transmission with number of particles simulated.

Tables 8.2-1,2,3 Percent of particles accelerated and \% losses with energies above 1 $\mathrm{MeV}$, for ideal waterbag input distributions with $5 \mathrm{~K}, 10 \mathrm{~K}, 100 \mathrm{~K}$ and $1 \mathrm{M}$ particles, and for the ion source emittance distribution of $\sim 1 \mathrm{M}$ particles rms matched to the RFQ input. 


\section{List of Acronyms}

$\begin{array}{ll}\text { AltCDR } & \text { An alternative CDR design } \\ \text { CDA } & \text { Conceptual Design Activity } \\ \text { CDR } & \text { Conceptual Reference Design } \\ \text { cw } & \text { Continuous-wave (100\% duty factor) } \\ \text { ECR } & \text { Electron cyclotron resonance } \\ \text { EP } & \text { Equipartitioned, a beam equilibrium state } \\ \text { HEBT } & \text { High-energy-beam-transport } \\ \text { IAP } & \text { Institut für Angewandte Physik, Goethe Uni Frankfurt, Germany } \\ \text { IFMIF } & \text { International Fusion Materials Irradiation Facility } \\ \text { IHEP } & \text { Inst. High energy Physics, Protvino, Russia } \\ \text { K } & \text { Thousand (10^3) } \\ \text { KP } & \text { Kilpatrick, developer of a voltage breakdown criterion. Here KP } \\ & \text { indicates the factor by which Kilpatrick's Criterion can be exceeded. } \\ \text { LEBT } & \text { Low-energy-beam-transport } \\ \text { LEDA } & \text { Low energy Demonstration Accelerator } \\ \text { LIDOS } & \text { RFQ design and simulation code, Moscow Radiotechnical Institute } \\ \text { M } & \text { Million (10^6) } \\ \text { m } & \text { meter } \\ \text { MeV } & \text { Million electron volts } \\ \text { MW } & \text { Megawatts } \\ \text { PARMTEQ(M) } & \text { RFQ simulation code, Los Alamos Scientific Laboratory } \\ \text { Post-CDR } & \text { A new design generated after publication of the CDR } \\ \text { RF } & \text { Radiofrequency } \\ \text { RFQ } & \text { Radio-Frequency Quadrupole } \\ \text { Rho/rO } & \text { Ratio of vane tip radius (Rho) to average aperture (rO) } \\ \text { Rs } & \text { Rf shunt impedance } \equiv(\text { vane voltage^2/(rf power) } \\ \text { TOUTATIS } & \text { RFQ simulation code, CEA Saclay } \\ \alpha & \text { Alpha ellipse parameter } \\ \beta & \text { Beta ellipse parameter } \\ & \end{array}$




\begin{abstract}
The IFMIF $125 \mathrm{~mA} \mathrm{cw} 40 \mathrm{MeV}$ accelerators will set an intensity record. Minimization of particle loss along the accelerator is a top-level requirement and requires sophisticated design intimately relating the accelerated beam and the accelerator structure. Such design technique, based on the space-charge physics of linear accelerators (linacs), is used in this report in the development of conceptual designs for the Radio-Frequency-Quadrupole (RFQ) section of the IFMIF accelerators. Design comparisons are given for the IFMIF CDR Equipartitioned RFQ, a CDR Alternative RFQ, and new IFMIF Post-CDR Equipartitioned RFQ designs. Design strategies are illustrated for combining several desirable characteristics, prioritized as minimum beam loss at energies above $\sim 1 \mathrm{MeV}$, low rf power, low peak field, short length, high percentage of accelerated particles. The CDR design has $\sim 0.073 \%$ losses above $1 \mathrm{MeV}$, requires $\sim 1.1 \mathrm{MW}$ rf structure power, has KP factor 1.7 ,is $12.3 \mathrm{~m}$ long, and accelerates $\sim 89.6 \%$ of the input beam. A new Post-CDR design has $\sim 0.077 \%$ losses above $1 \mathrm{MeV}$, requires $\sim 1.1 \mathrm{MW}$ rf structure power, has KP factor 1.7 and $\sim 8 \mathrm{~m}$ length, and accelerates $\sim 97 \%$ of the input beam. A complete background for the designs is given, and comparisons are made. Beam-loss distributions are used as input for nuclear physics simulations of radioactivity effects in the IFMIF accelerator hall, to give information for shielding, radiation safety and maintenance design. Beam-loss distributions resulting from a $\sim 1 \mathrm{M}$ particle input distribution representative of the IFMIF ECR ion source are presented.

The simulations reported were performed with a consistent family of codes. Relevant comparison with other codes has not been possible as their source code is not available. Certain differences have been noted but are not consistent over a broad range of designs and parameter range. The exact transmission found by any of these codes should be treated as indicative, as each has various sensitivities in its internal methods. Continued work to compare results between different codes more broadly and deeply than heretofore is highly recommended - this requires comparison at source code level and devising of appropriate tests. It is strongly recommended that the project obtain source code for all important simulation work.
\end{abstract}




\section{Introduction}

Progress toward the realization of fusion power requires development of lowactivation and neutron-damage resistant materials, with experimental suitability evidence satisfying technical and licensing requirements. Decades of work have established that a high-flux source of neutrons with appropriate spectrum must be built and operated, and that a neutron source based on the D-Li stripping reaction best suits this purpose. Facility design was first developed by the Fusion Materials Irradiation Test (FMIT) Project (1978-84) [1], later by the Energy Selective Neutron Irradiation Test Facility (ESNIT) Program (1988-92) [2], and from 1994-2006 by the International Fusion Materials Irradiation Facility (IFMIF) Project [3]. Major worldwide advances in accelerator technology over the past decade have further added to the credibility of this approach.

This report has been written for the IFMIF Project, as a United States contribution to EU Work Package TW5-TTMI-001, Task Deliverable 3, and complements extensive 2006 work by the Institut Angewandte Physik (IAP), Goethe Uni. Frankfurt on endto-end simulation, beam loss modeling, and the potential advantages of superconducting technology for the IFMIF linac.

IFMIF uses two continuous-wave (cw) $175 \mathrm{MHz}$ linear accelerators, each providing a $125 \mathrm{~mA}, 40 \mathrm{MeV}$ deuteron beam. The top-level performance requirements for the IFMIF accelerators are described in Table 1-1. Many aspects of the design are driven by the requirement that hands-on maintenance of the accelerator must be allowed throughout the life of the facility.

Table 1-1. Top-level specification for the IFMIF Accelerator Facility

\section{IFMIF Accelerator Facilities}

Performance requirements

Particle type

Accelerator type

Number of accelerators

Beam distribution

Output energy

Output energy dispersion

Duty factor

Availability

Maintainability

Design lifetime
$\mathrm{D}^{+} ; \mathrm{H}_{2}^{+}$for testing (to avoid activation)

RF linac

2 , in parallel operation

Rectangular flat top

(20 $\mathrm{cm}$ horizontal $\times 5 \mathrm{~cm}$ vertical)

$40 \mathrm{MeV}$

$\pm 0.5 \mathrm{MeV}$ FWHM

$\mathrm{CW}$ (pulsed tune-up and start-up)

$\geq 88 \%$

Hands-on (For accelerator components up to final bend in HEBT with local shielding as required; design not to preclude capability for remote maintenance.)

30 years

A Radio-Frequency Quadrupole (RFQ) accelerates the beam from $95 \mathrm{keV}$ to $5 \mathrm{MeV}$ and bunches the dc beam from the injector as required for injection into the following linac, which continues the acceleration to $40 \mathrm{MeV}$. The top-level 
specification for the RFQ is given in Table 1-2. The maintainability specification is a deceptively simple statement to the non-expert - it involves stringent minimization of stray particle loss along the linac; it is the crux of the design, and has been the subject of many years of research effort. This report strives to explore this aspect in breadth and depth, and therefore assumes, already from this point on, that the reader is familiar with the general description and elementary theory of the RFQ $[4,5,6]$.

Table 1-2. Top-level specification for the IFMIF RFQ.

\begin{tabular}{ll}
\hline Radio Frequency Quadrupole & \\
\cline { 2 - 2 } RFQ type & Resonant longitudinal coupling (3 RF segments), 4-vane integral \\
& structure \\
RF operating frequency & $175 \mathrm{MHz}$ \\
Input / output energy & $95 \mathrm{keV} / 5 \mathrm{MeV}$ \\
Input / output current & $130-140 \mathrm{~mA} / 125 \mathrm{~mA}$ (nearly all losses below 2 MeV) \\
RFQ length & $\sim 12.5 \mathrm{~m}$ \\
Total input RF power & $1.3 \mathrm{MW}$ \\
Cavity power & $685 \mathrm{~kW}$ \\
Transverse emittance & $\leq 0.4 \pi$ mm mrad (normalized rms) \\
Longitudinal emittance & $\leq 0.8 \pi$ mm mrad (normalized rms) \\
Duty factor & $\mathrm{CW}$ (pulsed tune-up and start-up) \\
Maintainability & Hands-on. May require local shielding. Design not to preclude remote \\
& maintainability.
\end{tabular}

The report compares in some depth three RFQs - the CDR Equipartitioned RFQ, a CDR Alternative RFQ, and a new Post-CDR Equipartitioned RFQ design - concerning the design strategies and trade-offs, the resulting designs and their performance in terms of beam loss:

- The CDR RFQ is an equipartitioned design $\sim 12 \mathrm{~m}$ long. The design strategy is described in detail in [7].

- An alternative CDR design uses a simpler design technique and had an additional design objective - to reduce peak surface field. It is also $\sim 12 \mathrm{~m}$ long, and has about the same transmission and loss features as the CDR RFQ although crossing a parametric resonance.

- Refined equipartitioned design and optimization techniques result in a Post-CDR design. The beam loss at energies above $\sim 1 \mathrm{MeV}$, rf power and peak field are similar to the older designs, while the length is reduced to $\sim 8 \mathrm{~m}$ (significant cost saving) and the percentage of accelerated beam is significantly higher.

The decision to use the equipartitioned RFQ design for the CDR is recorded in [8]. This report gives background and further development.

Although this report presents three RFQ designs and beam-loss distributions, any one of these should not be considered as a final design. The intent of these designs has been to develop improved design and optimization techniques, and to explore design strategy options. Using these tools and from these examples, a final design 
can be decided. Very substantial work is required for the final design. Issues include:

\subsection{Caveats}

\subsubsection{LEBT Design and Simulation Refinement}

The low-energy-beam-transport (LEBT) design has a very strong influence on the RFQ design (beam loss and transmission performance, length, rf power consumption). The CDR presents a feasible conceptual case; a fully integrated and optimized LEBT/RFQ combination for IFMIF has not been designed yet.

The choice of injection energy into the RFQ is important. Typically, a lower injection energy results in a shorter RFQ but other aspects of the RFQ performance may suffer. Lower injection energy results in higher space-charge effect in the sections of the LEBT which are not space-charge neutralized. Studies during the IFMIF project led to the choice of $0.095 \mathrm{MeV}$ injection energy presented in the CDR. This choice was re-evaluated; the conclusion is that $0.095 \mathrm{MeV}$ appears optimal.

A much more accurate representation of the ion-source/LEBT is required to fully characterize the beam distribution entering the RFQ. The best beam-loss model produced so far is described in the CDA [9] Issues include:

- ECR ion source performance at the require 130-140 mA deuteron current with the specified emittance has not been demonstrated. Scaling to $125 \mathrm{~mA}$ deuterons requires $200 \mathrm{~mA} \mathrm{H}+$ from the source; this level has not been demonstrated and the corresponding emittance is not known.

- There is a paucity of emittance data from the ECR ion source; only one 125 $\mathrm{mA} \mathrm{H}+$ emittance data set has been available to the Project, and no deuteron beam emittance measurement. This data set has been numerically transformed to the input ellipse matching parameters required by the RFQs. However, this data set is thresholded by an inaccurate (and optimistic) method. The more accurate method developed by M. Stockli at SNS should be used. [10]

- The beam is composed of multiple species of deuteron ions (and may also be contaminated with other ions). The emittance data need to be separated according to species, and all species tracked simultaneously through the LEBT and the RFQ. (The simulation program pteqHI can transport multiple species simultaneously.)

Other LEBT effects include:

- beam neutralization ( 98\%),

- high-frequency noise on the ion-source beam. This noise may result in an amplified beam current variation in the LEBT if the time constant of the neutralization phenomenon is slower than the noise, and this effect must be included in the simulation. $[9,11]$

- a short $(\sim 3 \mathrm{~cm})$ section at the RFQ input where there is neither neutralization nor focusing, resulting in a strong space-charge effect.

- The 2-solenoid low-energy-beam transport (LEBT) between the ion source and the RFQ has not been designed in detail and is not included in the input beam distribution used in this report. The solenoid simulation should include higher- 
order field effects both within the solenoid and in the solenoid end fringe field regions, which will produce further aberrations in the input beam. [12]

- Coulomb scattering of beam particles on residual gas [13]

- Effect of auxiliary gasses [14]

These issues are being addressed by IAP, Goethe Uni Frankfurt in the same EU 2006 Work Package; these results and method for presenting beam loss should be used in the final RFQ design work.

These effects will probably produce larger beam-losses in the presented RFQ designs. As the RFQ design is intimately related to the input beam, it may be possible to reduce the RFQ losses again with further optimization work using the more accurate ion-source/LEBT input distribution in the optimization process.

\subsubsection{HEBT Design and Simulation}

Conceptual design of the IMFIF High-Energy-Beam-Transport, from the end of the linac to the target, is not complete and presents design challenges, which may be influenced by the linac performance, including errors.

\subsubsection{RFQ Design Strategy, Trade-offs, Optimization}

- The RFQs presented here require detailed design work to add vane gaps between manufactured sections, and the transition and output radial matching cells at the output.

- The final choice of parameters and design strategy has to ultimately be made by the IFMIF program - selecting the desired trade-off between the top-level specifications for low beam loss, cost factors such as RFQ length and rf power requirement, and engineering factors such as peak field. In this report, a prioritization of the top-level specs has been chosen:

1) Minimum beam loss at energies above $\sim 1 \mathrm{MeV}$. This has been chosen as the highest priority because maintenance of the linac without the use of remote manipulators ("hands-on" maintenance) is of primary importance. There is no lower energy threshold for inducement of radioactivity when deuterons strike material, but confinement of beam losses as much as possible to under $1 \mathrm{MeV}(\sim 10$ times the injection energy) can be achieved with design and optimization techniques.

2) Low rf power requirement for the RFQ structure. The cost of rf power comprises the initial capital cost plus the operating cost over the factory life, and therefore overshadows the capital cost of the RFQ structure.

3) Low peak field. If the peak field were too high, sparking and performance degradation could occur. This requirement is not so difficult to achieve, as the required beam focusing can be achieved without needing the highest practical fields.

4) Short length. Modern RFQs can be built to any length. For IFMIF, considerations of the beam acceleration and efficiency have guided the choice of 5 $\mathrm{MeV}$ as the output energy of the RFQ. The RFQ capital cost is then optimized for the shortest RFQ that accelerates from $0.095-5 \mathrm{MeV}$, while meeting the other specs.

5) High percentage of accelerated particles. The IFMIF ECR deuteron ion source should reliably produce at least $140 \mathrm{~mA}$ cw beam current, and $125 \mathrm{~mA}(89.3 \%)$ must be accelerated. Better would be $\sim 96.2 \%$ acceleration of $130 \mathrm{~mA}$ input, and this has been a design goal of the CDR and Post-CDR work. Some sacrifice of 
accelerated beam percentage could be made, assuming the ion source should have some excess capacity, in order to minimize $>1 \mathrm{MeV}$ losses; i.e., transmission is hardly the full story.

- Then the final design must be optimized, including sensitivity checks of various factors (e.g., different input emittance, beam current and matching). Guidance is provided in this report. At present, the optimization procedure is still tedious.

- It is important to recall also that IFMIF decided for multiple linacs for increased reliability of beam on target. Each linac would provide $125 \mathrm{~mA} \mathrm{cw}$ beam, with the present IFMIF consisting of two such modules. A later upgrade of the module for higher beam current would not be done; if higher current were desired, another module would be installed. This strategy then allows module performance and cost optimization, which guided the choice of main parameters such as the $\mathrm{rf}$ frequency.

- New insights into factors influencing optimum transmission and low beam loss have been gained and are the subject of ongoing research; it is hoped they will be useful in the final design work for IFMIF.

\subsubsection{Design and Simulation Tools}

- The LINACS design tool $[15,16]$ used in this report gives complete control over the space-charge physics, including utilization of an equilibrium beam [17], control of the tune trajectory with respect to resonances, and control of all parameter variations inside the RFQ.

- Simulation results presented in this report were obtained using the pteqHI code [18], a version of PARMTEQ with many improvements, including the ability to simultaneously handle multiple species. Multipole and image effects are represented analytically using the method of Crandall $[19,20]$. The source code is available. Final design work should use a code with similar improvements and also a field map representation of the vane surfaces. The RFQTRAK [21] or LIDOS [22] codes are recommended. The newest version of PARMTEQM also uses field maps, but continues to use an approximate method for space-charge computation (the independent variable is position instead of time, transformations at the spacecharge computation points are inaccurate, resulting in inaccurate beam loss localization (beam-loss pattern) [12] ). Despite arduous attempts, reproduction of published results of the TOUTATIS code [23] was not successful, and therefore it has not been used further.

The exact transmission found by any of these codes should be treated as indicative, as each has various sensitivities in its internal methods. Continued work to compare results between different codes more broadly and deeply than heretofore is highly recommended - this requires comparison at source code level and devising of appropriate tests. It is strongly recommended that the project obtain source code for all important simulation work. 


\subsection{Outline of the Report}

The report has two main purposes:

1) (Sections 2-3) To present three designs in the usual format of:

- their vane parameters vs. cell number

- their transmission

- their beam-loss pattern.

2) (Sections 4-9) To go beyond the usual format,to:

- explain in some depth the development of linac design technique, and how this influences the three designs.

- explain enhanced design and simulation tools that afford control of the beam space-charge physics and the design strategy and parameters.

- analyze the designs in these terms.

- give comments on a varying vane-voltage profile, the rf power estimates, the design and simulation codes, and the LEBT.

Section 2 presents and compares the vane parameter characteristics and basic rms beam behavior of each of the three RFQs.

Section 3 presents, under restricted conditions, beam-loss distributions for each of the RFQs.

Section 4 outlines the process of "conventional" RFQ design technique, exemplified by the Alternative CDR RFQ.

Section 5 discusses Beam-Based Linear Accelerator Design Technique, of which RFQ design is a subset, exemplified by the CDR and Post-CDR RFQs,

Section 6 presents and compares the performance of the three example RFQs against the background of beam-based design. The underlying space-charge physics of the beam as it travels through the RFQs is revealed. This is the key section, with a summary and suggestions for further design work.

Section 7 discusses the use of a variable vane voltage profile, which has been used by experienced RFQ designers in operating RFQs. The characteristics of the Russian IFMIF partner's preliminary proposal for an RFQ are given as an example of support for variable vane voltage.

Section 8 characterizes the computer codes used for design and simulation, upon which the results for all three designs critically depend. Variation of results with the number of particles simulated is presented.

The material in Sections 5-8 goes beyond the commonly used practice, and must be understood by project team members, as well as project leaders, reviewers and other interested parties.

In capsule form: "Conventional" design techniques involve a global characterization of space charge defocusing vs. rf field focusing in terms of a "current limit" and simplified rules for parameter variation. The advanced beam-based technique requires definition of the detailed space-charge defocusing vs. rf field focusing at 
each cell, and then finds the appropriate RFQ structure to satisfy this space-charge physics definition. There are more parameters available than needed to satisfy the space-charge physics requirements; the extra ones are specified by rules. All of the many RFQ parameters are under direct control of the designer.

Accurate beam distributions for the ion-source and simulation through the LEBT are crucial for accurate simulation through the RFQ and post-RFQ linacs to the full energy of $40 \mathrm{MeV}$, as discussed in Section 1.1; a few additional notes are given in Section 9.

Section 10 gives a few concluding remarks. 


\section{Presentation of Three IFMIF RFQ Designs}

The figures are traditional for RFQ experts, and are given here without further elaboration.

\subsection{CDR Equipartitioned RFQ}

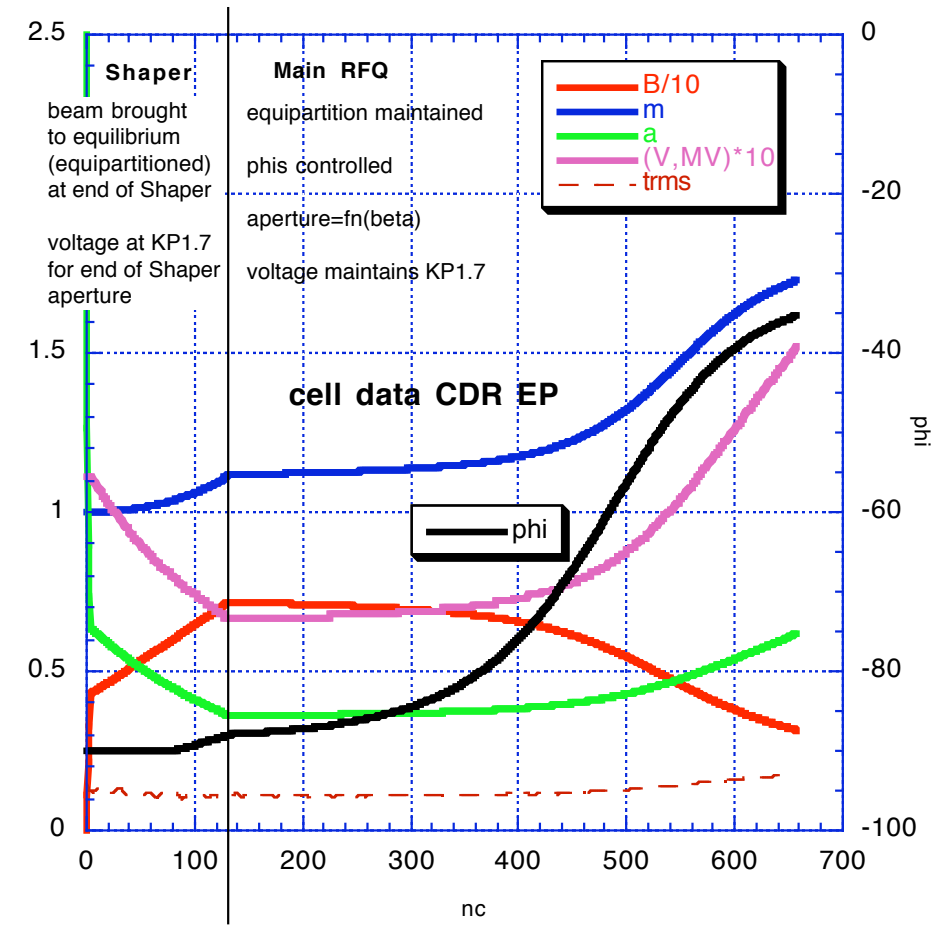

Fig. 2.1-1. Vane parameters for the $140 \mathrm{~mA}$ CDR equipartitioned RFQ. Aperture (a) and trms $=$ transverse $\mathrm{rms}$ beam radius are in $\mathrm{cm}$. (m) is the vane modulation, $\mathrm{V}$ is the vane voltage, $\mathrm{B}$ is the transverse focusing strength. $\mathrm{Phi}$ is the synchronous phase (phis). Rho/rO $=0.75$. Input current $=140 \mathrm{~mA}$, input energy $=0.100 \mathrm{MeV}$, input transverse normalized $\mathrm{rms}$ emittance $=0.20$ mm.mrad. 


\subsection{CDR Alternative RFQ}

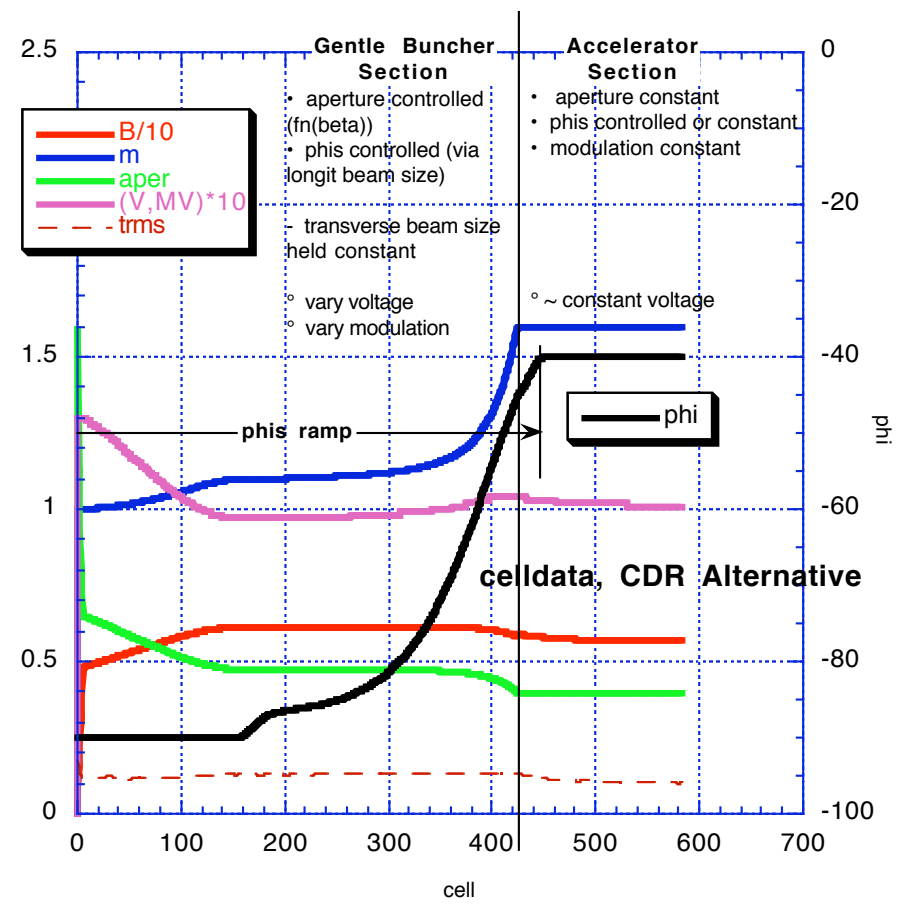

Fig. 2.2-1. Vane parameters for the $130 \mathrm{~mA} C D R$ Alternative RFQ. Input current $=130 \mathrm{~mA}$, input energy $=0.095 \mathrm{MeV}$, input transverse normalized $\mathrm{rms}$ emittance $=0.25 \mathrm{~mm} \cdot \mathrm{mrad}$.

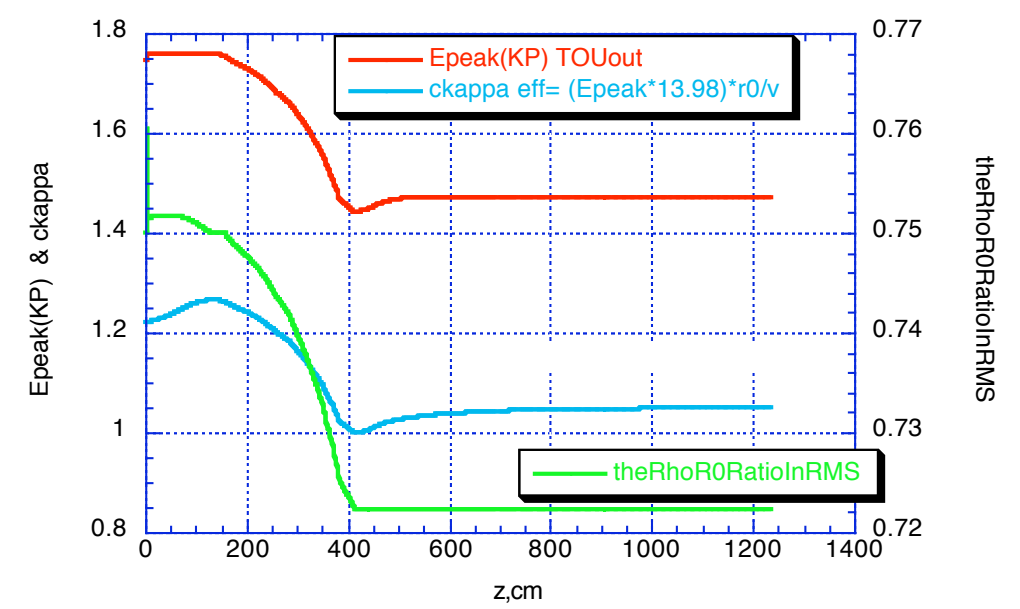

Fig. 2.2-2. The Rho/r0 ratio is varied, to give lower peak surface field (lower KP factor) in the downstream part of the RFQ. Epeak (KP) is the KP factor, ckappa is the ratio between the peak field on the vane surface and the vane-tip field. 


\subsection{New Post-CDR Equipartitioned RFQ}

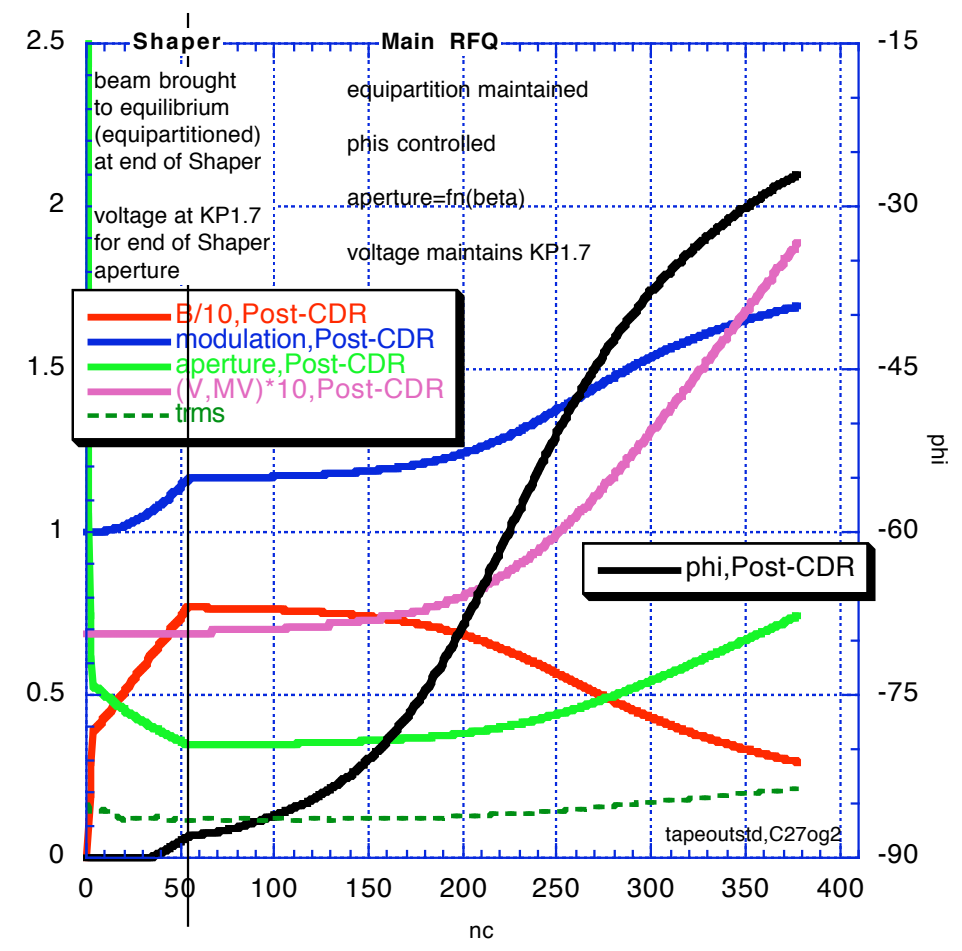

Fig. 2.3-1. Vane parameters for the $130 \mathrm{~mA}$ New Post-CDR RFQ. Input current $=130 \mathrm{~mA}$, input energy $=0.095 \mathrm{MeV}$, input transverse normalized rms emittance $=0.25 \mathrm{~mm} \cdot \mathrm{mrad}$. 


\subsection{Comparisons}
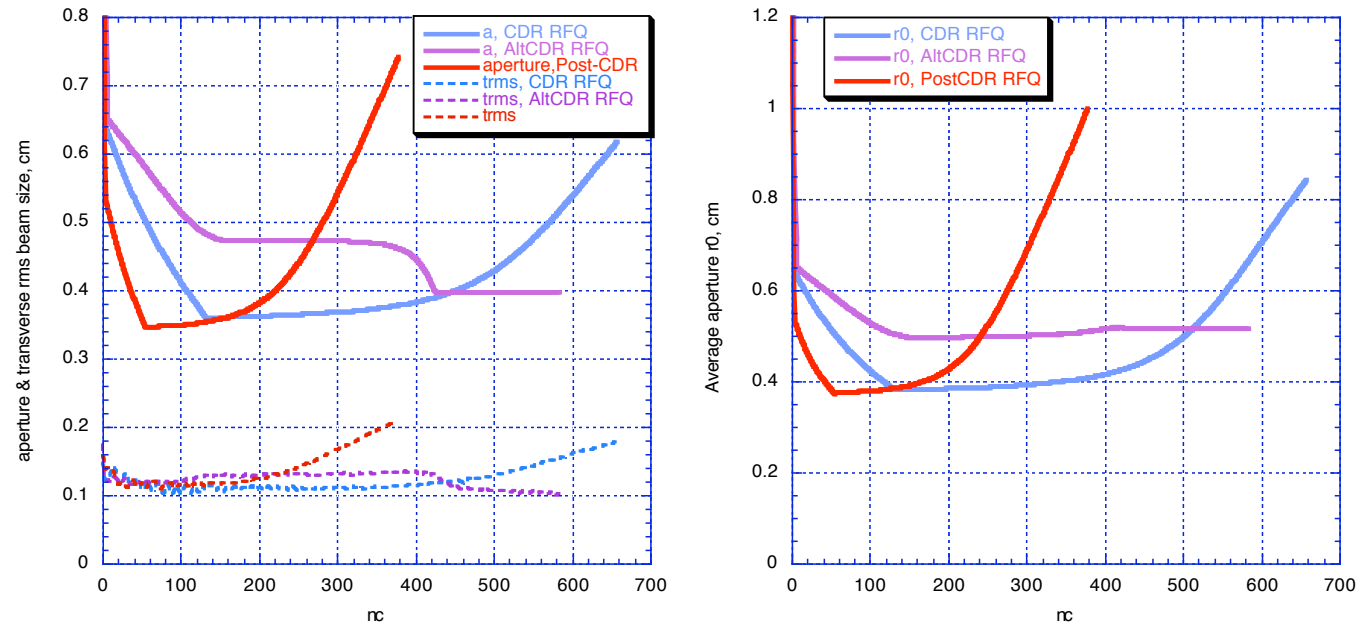

Fig. 2.4-1,2. Comparison of minimum aperture, a (nearly the same for the three RFQs), and average aperture, r0.
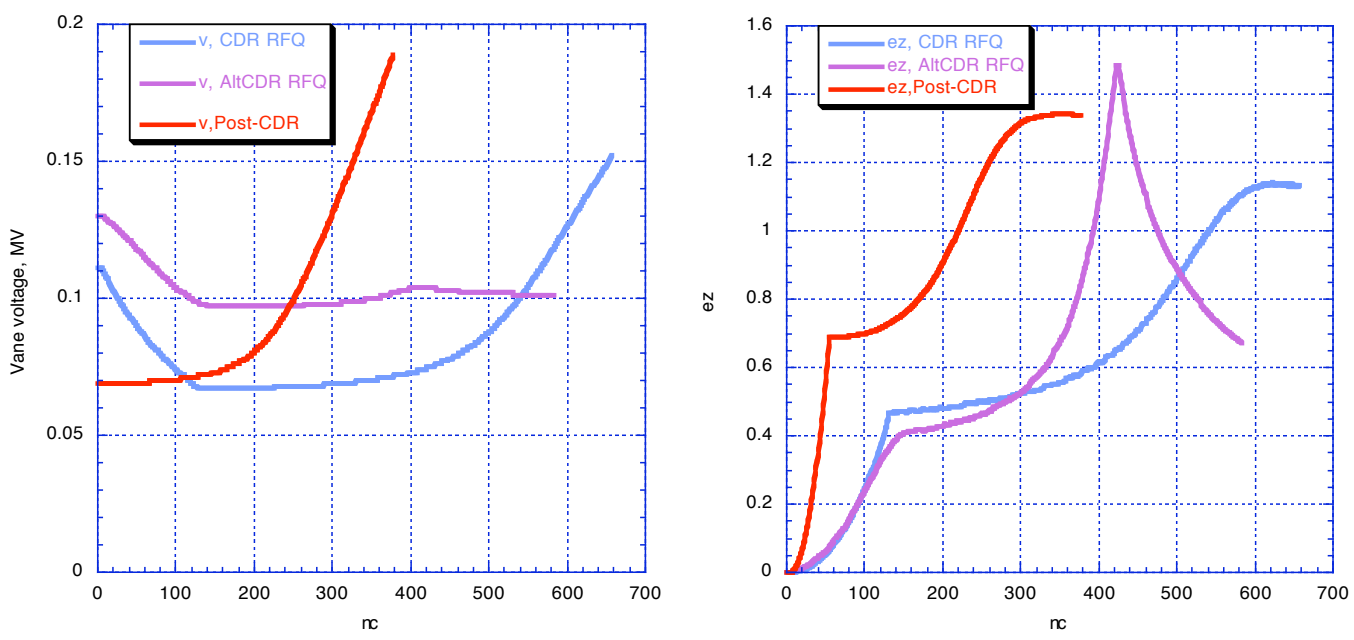

Fig.2.4-3,4. Comparison of vane voltage and accelerating factor ez. 

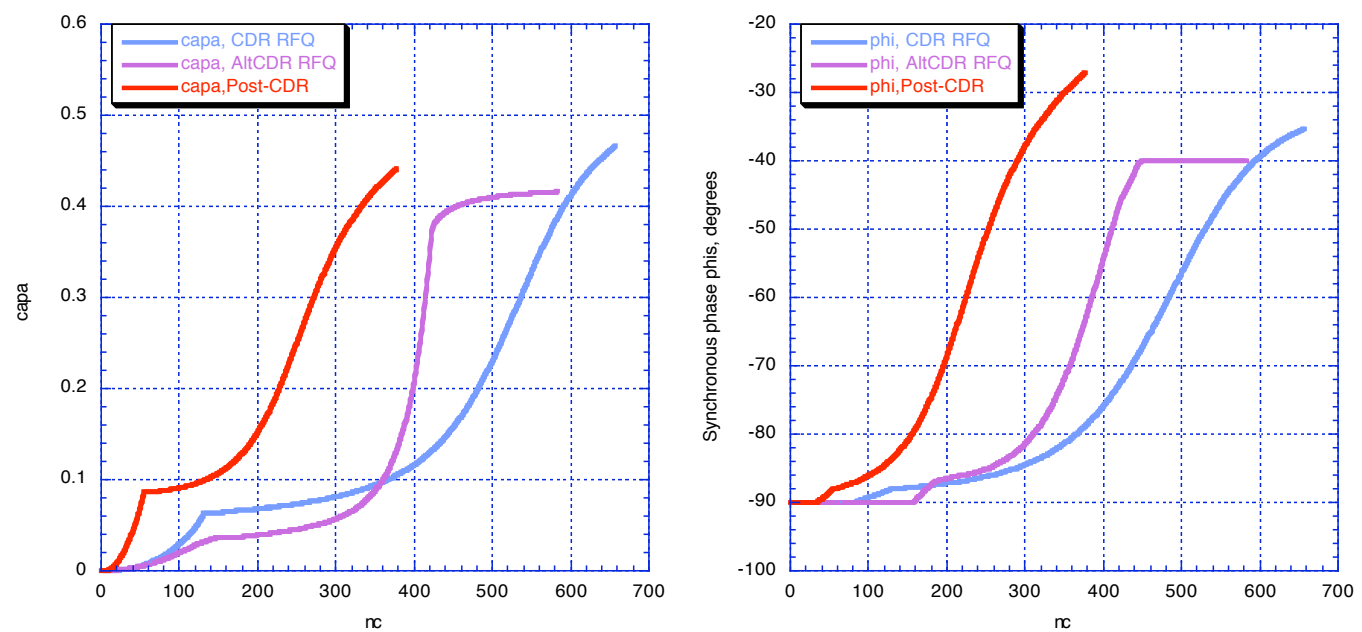

Fig. 2.4-4,5. Comparison of accelerating efficiency A and synchronous phase phis.
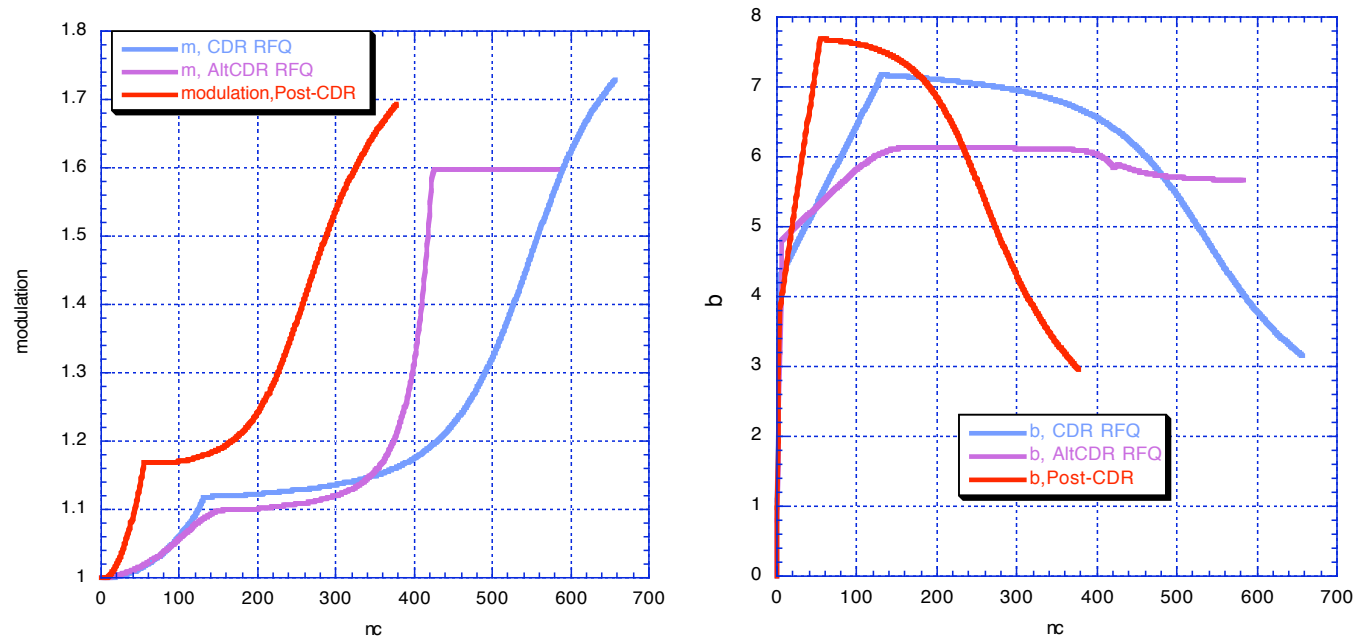

Fig. 2.4-7,8. Comparison of vane modulation $\mathrm{m}$ and focusing factor $\mathrm{B}$.
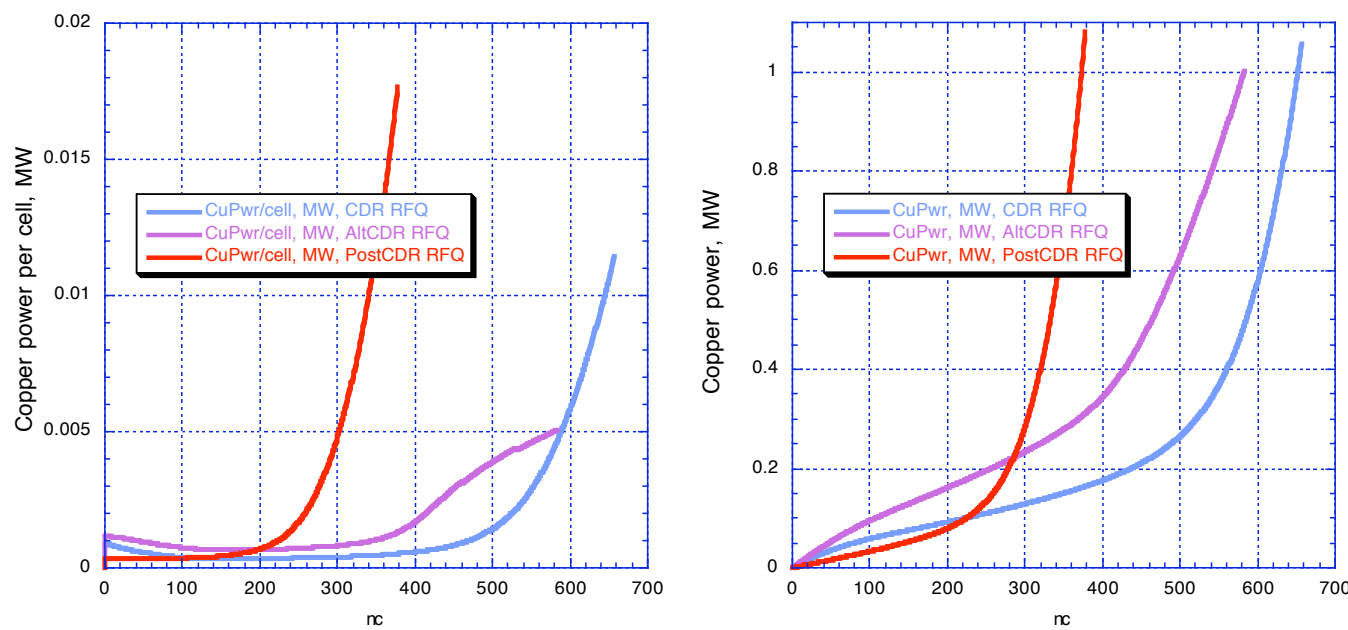

Fig. 2.4-9,10. Comparison of Copper power per cell and integrated copper power. 


\section{Beam-Loss Distributions and Accelerated Beam Transmission}

Some particles are inevitably lost in a particle accelerator; their reactions with the surrounding materials produce radioactive by-products, accumulation of which must be minimized. The location and energy of lost particles is obtained from simulations of the accelerator, and used as input for nuclear physics simulations of radioactivity effects in the IFMIF accelerator hall, to give information for shielding, radiation safety and maintenance design. Conservative safety margins are assigned in each of these areas over the simulated effects.

Simulations were run with up to $1 \mathrm{M}$ particles, including a representative distribution from the ECR ion source. Only the results using this ion source distribution are presented in this Section. It is necessary to build up extensive background in Sections 4 and 5, before discussion of the beam performance can be given in Section 6.

The definitions applied in the accelerator simulation to determine when a particle is assumed lost, and with what energy, are outlined in Section 8.2.2.

\subsection{Beam Loss Distribution Comparisons}

Confinement of losses to low energy as much as possible helps reduce the induced radioactivity, and this was chosen as the most important of the five main

specifications. At the design conditions, the three RFQs are rather similar; for the ion source emittance distribution of $\sim 1 \mathrm{M}$ particles $\mathrm{rms}$ matched to the RFQ input, the percent of particles lost with energy $\geq 1 \mathrm{MeV}$ is:

$\begin{array}{ll}\text { CDR } & 0.073 \% \text { loss above } 1 \mathrm{MeV} \\ \text { AltCdr } & 0.123 \% \text { loss above } 1 \mathrm{MeV} \\ \text { Post-CDR } & 0.081 \% \text { loss above } 1 \mathrm{MeV}\end{array}$

Although the totals are similar, Figs. 3.1 and 3.2 show different loss distributions. This will be discussed later in Section 6 . 


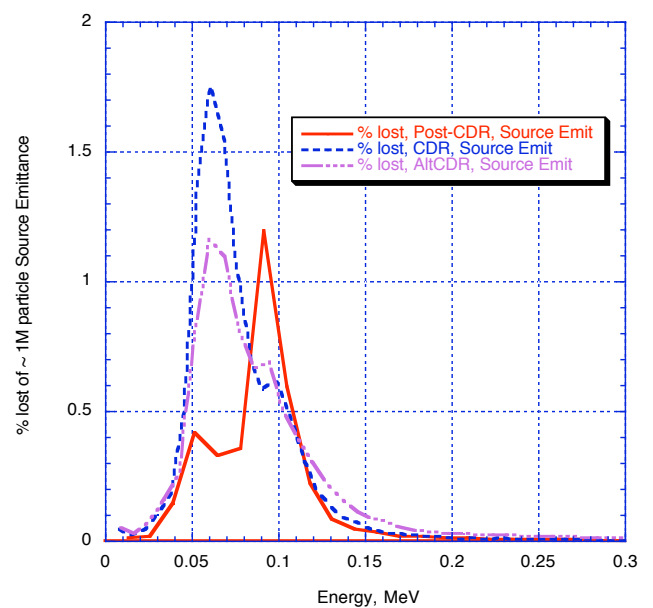

Fig. 3.1-1. \% of all lost particles vs. energy where lost, $0.0-0.3 \mathrm{MeV}$.

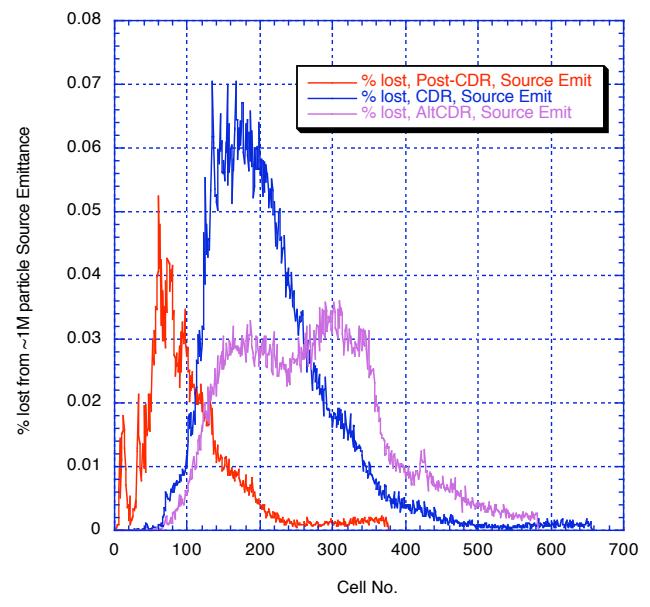

Fig. 3.1-2. \% of all lost particles vs. position $(z, \mathrm{~cm})$ where lost.
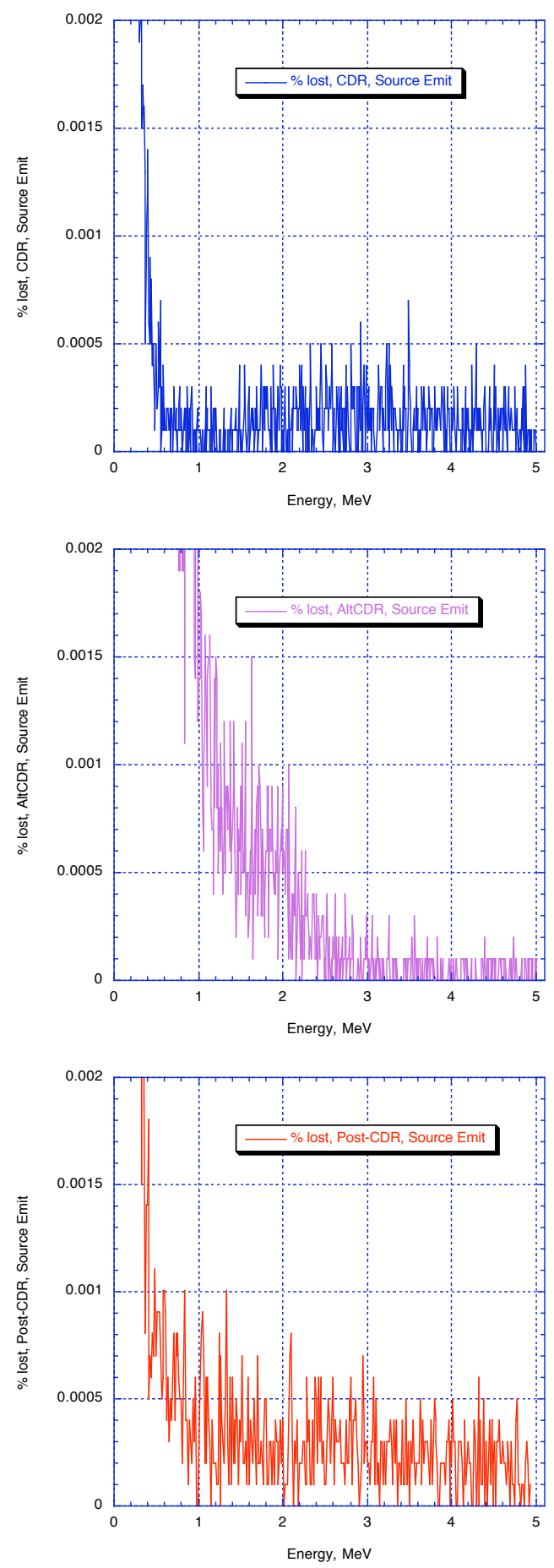

Fig. 3.1-3. \% of all lost particles vs. energy where lost, 0.0 - $5.05 \mathrm{MeV}$, expanded vertical scale. 


\subsection{Transmission to RFQ output}

For the ion source emittance distribution of $\sim 1 \mathrm{M}$ particles rms matched to the RFQ input, the percent of accelerated beam is:

$\begin{array}{ll}\text { CDR } & 89.4 \% \\ \text { AltCdr } & 89.9 \% \\ \text { Post-CDR } & 95.8 \%\end{array}$

Fig. 3.2-1 shows the percent of accelerated particles through the RFQs. Features will be discussed in Section 6 .

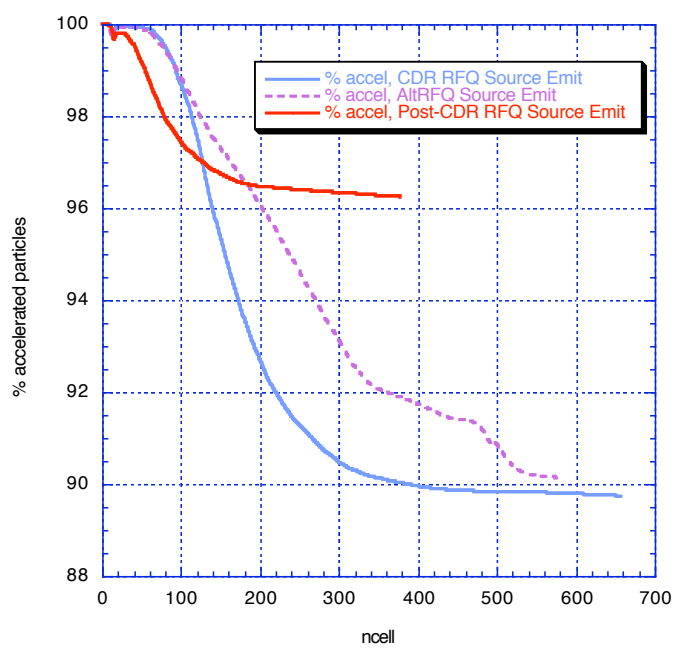

Fig. 3.2-1. \% Accelerated particles from Source Emittance initial distribution vs cell number.

The total percentage of transmitted particles, including particles with low energy, is:

$\begin{array}{ll}\text { CDR } & 90.0 \% \\ \text { AltCdr } & 92.0 \% \\ \text { Post-CDR } & 97.0 \%\end{array}$




\section{4. "Conventional" RFQ Design Technique}

The job of a linac is to efficiently accelerate a beam of given current from an initial energy to a final energy. If some particles are lost by striking the accelerator structure during this process, they will cause a radioactivity buildup over time - thus minimization of beam loss becomes an overriding concern. It would not be economically feasible to maintain an entire linac by remote manipulators, so the specification requires "hands-on maintenance," meaning there can be local hot spots that may require special tooling, shielding, and limited access, but not remote manipulators.

"Conventional" intense RFQ design, still used almost exclusively today, is exemplified by the design of RFQs as originally laid out by Los Alamos in 1978-1980, in which only a global beam-current-related criterion was invoked for guidance as to the practical maximum current that should be accelerated for given focusing parameters, plus a cellby-cell recipe for assigning the synchronous phase. The linac is laid out, keeping many parameters constant in the main accelerating section to ease manufacturing and tuning as understood at the time. Then beam acceleration is simulated and the resulting transmission to the output is observed as the measure of successful performance. It has been considered "too difficult" to understand in detail the cell-by-cell performance of the beam as it undergoes the very complicated process of forming a bunch from an initially dc beam and accelerating. This method is thus characterized as design "from the external field to the beam result" - i.e., "from the outside in".

\subsection{External Field Quantities as Defined by the RFQ Metal}

\subsubsection{2-Term Potential Field Description}

The RFQ is an accelerating device with time-varying fields. The classical description of the RFQ external fields uses the first two terms of the field solution in the vicinity of the vane tips - called the ' 2 -term potential' - to describe the transverse and longitudinal field. It is emphasized that this representation is only accurate near the beam axis. In practice, this has led to the use of field maps in recent codes (LIDOS, the most recent version of PARMTEQM, and TOUTATIS).

The metal contour of the vane in the 2-term potential description is described by the aperture between opposing vanes and the modulation in the longitudinal direction. A potential, which can vary with the position $(z)$ along the RFQ, is set up between the opposing pairs of vanes by the rf field, to provide the focusing and accelerating fields. Unmodulated vanes produce a purely transverse focusing field. Perturbation of the vanes with a specific, velocity-modulated pattern produces a longitudinal field component that can provide particle acceleration.

Modern practice replaces the 2-term longitudinal profile with a sinusoidal profile, which is easier to machine and gives somewhat more efficient acceleration. 


\subsubsection{Multipole and Image Fields}

In practice, the vanes are not machined to the exact 2-term potential shape; the compromises result in higher order multipole terms that must be included. Older versions of simulation codes handled the higher-order terms analytically with coefficient tables $[19,20]$; pteqHI incorporates this method.

Duperrier (24) showed, for fields including analytically expressed multipoles, that inaccuracies occur outside the transverse circle drawn at the vane tip minimum. That and removal of paraxial approximation were main reasons why he wrote the simulation code TOUTATIS.

Recent codes (LIDOS, the most recent version of PARMTEQM, and TOUTATIS) incorporate field maps, which represent the exact vane shape used, out to some radius from the axis.

Image-charge effects are important in RFQs with significant beam current. The analytical method of Crandall [20] is implemented in both the design code LINACSrfq (negligible in the envelope design process, but coefficient tables are produced for the simulation code pteqHI), and in pteqHI.

\subsection{Minimum Beam-Related Specification}

Four variables describe the RFQ vane surface:

- either the minimum aperture $\mathrm{a}[\mathrm{rfq}]$ or the average aperture $\mathrm{r} 0[\mathrm{rfq}]$ :

- the modulation em[rfq]

- the voltage between opposing vane tips v[rfq]

- the synchronous phase angle phis[rfq] at the location of the synchronous particle in each cell when the rf phase equals zero.

\subsubsection{Teplyakov Synchronous Phase Rule}

The synchronous phase angle must be specified. This is usually done using the rule invented by Teplyakov [25] which was the key to the success of the RFQ: the charge density of the forming bunch should remain constant; i.e., the ratio between the accelerating bucket length and the beam length should be constant. In "conventional" design procedures, this is the only place where a relationship between the beam and the structure is required at each cell.

The charge density can be allowed to vary to some extent, and specific control is available in the more flexible design procedure available in LINACS. This control of the charge density is especially useful in controlling the length of the RFQ.

\subsubsection{Beam-Envelope Matching}

Two envelope equations describe the variation of the rms beam radius and length of a bunched ellipsoidal beam in the smooth approximation (rapid variation of the quadrupolar field is smoothed) of a focusing system, as a function of the external fields and the internal space-charge forces within the beam, which work to counteract the external fields: 


$$
\begin{aligned}
\varepsilon_{t n} & =\frac{a^{2} \sigma_{t} \gamma}{\lambda} \\
\varepsilon_{\ln } & =\frac{(\gamma b)^{2} \sigma_{l} \gamma}{\lambda}
\end{aligned}
$$

where $\varepsilon$ is the normalized rms (root mean square) emittance, $\sigma$ is the phase advance with beam current, $t$ denotes transverse and $l$ longitudinal, $a$ and $b$ are transverse and longitudinal $\mathrm{rms}$ beam radii, $\lambda$ is the $\mathrm{rf}$ wavelength.

The emittance terms must be either constant, or with a variation known a-priori.

Expanding the phase advance (or "tune") terms;

$$
\begin{gathered}
\sigma^{t^{2}}=\sigma_{0}^{t^{2}}-\frac{I \lambda^{3}(1-f f)}{a^{2}(\gamma b) \gamma^{2}} k \\
\sigma_{l}^{2}=-\sigma_{0}^{l^{2}}-\frac{2 I \lambda^{3} f f}{a^{2}(\gamma b) \gamma^{2}} k
\end{gathered}
$$

$\sigma_{0}$ is the zero current phase advance:

$$
\begin{aligned}
& \sigma_{0}^{t^{2}}=\frac{B^{2}}{8 \pi}+\Delta \\
& \sigma_{0}^{l^{2}}=-2 \Delta
\end{aligned}
$$

where $\mathrm{B}$ is the RFQ focusing parameter, $I$ is the beam current, $f f$ is the geometry factor $\approx a / 3 b, \gamma$ and $\beta$ are the relativistic gamma and beta, and $k=\frac{3}{8 \pi} \frac{z_{o} q 10^{-6}}{m c^{2}} \cdot \Delta=\frac{\pi^{2} q V_{0} A \sin \phi_{s}}{2 m c^{2} \beta^{2}}$ is

the rf defocusing parameter, $A, V_{0}, \phi_{s}$ are accelerating parameter, intervane voltage and synchronous phase respectively.

These equations apply to a periodic focusing system of infinite length, but can be applied locally when the parameter variation is reasonably adiabatic, as indicated by the absence of first and second derivative terms. Careful checks were made from the full Hamiltonian to verify that terms related to acceleration are negligible (always assumed, but a reference has not been found). Equating the second derivatives of the beam sizes to zero means that the beam is "matched" to the focusing system. This condition is essentially satisfied in practical designs; however, there is evidence that in some subtle aspects the derivatives should be taken into account, and this is a subject of ongoing research.

\subsubsection{Beam-Envelope Matching at Transitions}

Transitions occur often in practical linacs; as energy increases, a different type of accelerator structure must be used, or engineering reasons require parameter changes, etc. The matching equations (1) and (2) immediately indicate how to maintain the matched condition at a transition, across which it would be desired that the emittances and beam sizes remain constant. Maintaining the matched conditions then requires that the phase advances per unit length, $\sigma / \lambda$, also remain constant. 


\subsubsection{Global Space-charge Rule}

If the current is increased until the phase advances equal zero, the "space-charge limit" is reached. At some intermediate current, the matching equations (1) and (2) indicate that the emittances and the current will have equal effect on the beam radii. If the beam bunch is assumed to be spherical, this occurs when the "tune depressions) $\sigma / \sigma 0=$ 0.4. The original procedure solved Eqs. (1) and (2) independently with $\sigma / \sigma 0=0.4$ to find "transverse and longitudinal current limits," which were brought to equality by parameter adjustments. Requiring that the desired operating current be $\sim$ half of this "current limit" was then used as a practical guide for RFQ design.

\section{3 "Conventional Design"}

The design process developed at Los Alamos in 1978-1980 is still the most commonly used. It basically approaches the problem from the point of view of the external fields, and then checking the effect on the beam using a full simulation code. The rule that the "current limit" is twice the desired operating current guides the choice of $\mathrm{rf}$ frequency, which in turn governs the maximum allowable intervane voltage according to a "bravery factor" (typically $\leq 2$ ) over a sparkdown criterion such as defined by Kilpatrick.

Parameters (such as the average aperture rO between vanes, the voltage $\mathrm{V}$ between opposing vanes, the transverse focusing parameter B) were held constant along the vanes as much as possible to ease manufacturing complexity. Later it was learned how the parameters could be varied, giving an additional element of flexibility to the design process.

The RFQ is divided into four sections. A "radial matching section" about 4-6 cells long brings the beam into the RFQ by ramping up the fields from zero to a chosen value. The fields are then raised in a "shaper" section to the full transverse focusing ability available from the voltage and aperture chosen (zero modulation and $-90^{\circ}$ synchronous phase). Then the beam is slowly bunched and accelerated in a "gentle buncher" section, being very careful not to create strong space-charge forces leading to beam blow-up and lost particles, either immediately or later downstream. Typically the gentle buncher accelerates to about ten times the injection energy. Reasonable optimization is achieved by varying the synchronous phase reached at the end of the gentle buncher section.

The average aperture, r0, was held constant, to facilitate tuning the structure, and this also keeps the transverse beam size $\sim$ constant as the modulation increases. This has an important consequence, because as the modulation, synchronous phase and beam velocity increase, the required minimum aperture decreases quickly toward zero, as seen from the relation $\mathrm{r} 0=($ Minimum Aperture $)(1+$ modulation $) / 2$. Before this can occur, further increase of the modulation must be stopped. This point nominally defines the end of the "gentle buncher" section, and is considered the "choke-point" where the "current-limit" requirement applies.

An improvement for intense beams was to modify the shaper and gentle buncher to have a long initial "porch" section where the synchronous phase remains at $-90^{\circ}$ with no modulation to allow initial bunching with no acceleration, followed by a section with 
increase of phis of a few degrees and small modulation to bring the beam to a satisfactory bunch length, and then using the Teplyakov rule for phis to further bunch and accelerate the beam.

The "acceleration section" then accelerates the beam to the final energy. The modulation is held constant at its end-of-gentle-buncher value. Within the constraint of the focusing available from the vane voltage such that $\mathrm{r} 0$ remains constant, the synchronous phase may be raised further, and then is clamped.

It was also found that a lower $\mathrm{B}$ at the beginning of the shaper requires less convergence of the injected beam, making the space-charge effects at injection less and making it easier to achieve a good input match. Therefore a lower vane voltage can also be used at the front end, lowering rf losses.

\subsection{1 "How to choose the operating point?"}

We draw here directly from material in [26] for an equivalent approach to define the end of the gentle buncher, as used in the design of the Alternative CDR RFQ:

"1) We know that the biggest problem should be expected at the end of the Gentle Buncher, when the bunch already exist but it is not really accelerated. At this point we know the energy of the beam, the phase, the emittance and the current.

2) We draw the following figure. There are two sets of curves. The dot curves (o) show the lines of a given Kilpatrick value (1.8 is the desired value in our case, the green curve). So we have to choose a point on that line. The second set is described by lines with $(+)$, representing the modulation value.

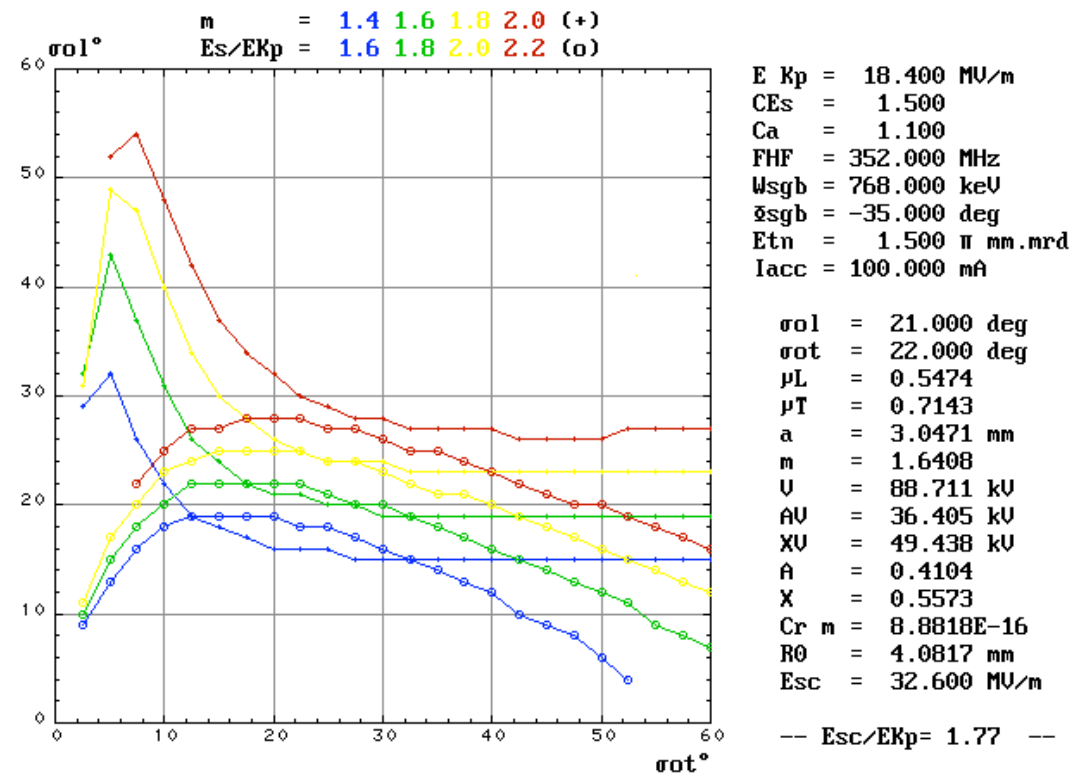

Fig. 4.1 A global design aid - 1. (Caption added.)

So, for a given Kilpatrick we choose the maximum modulation in order to obtain the smallest RFQ. In this particular figure, we have chosen $\sigma_{0 t}=22^{\circ}$ and $\sigma_{01}=21^{\circ}$, and this point give the modulation, aperture, vane voltage, depression tune $\mu$ t and $\mu$ l. 
3) We draw the next figure. It permits to verify that the chosen point is a valid one. We know that a " good» point will be with a depression tune over 0.4 . The red curve describes $\mu \mathrm{t}$ as a function of $\sigma_{0 t}$. We have chosen $22^{\circ}$, so we can verify that this value give us a $\mu$ t over 0.4 . The green curves are a set of curves describing $\mu \mathrm{l}$ as a function of $\sigma_{0 t}$ for different value of $\sigma_{01}$. We verify that the point $\sigma_{0 t}=22^{\circ}$ and $\sigma_{01}=21^{\circ}$ give us a $\mu 1$ over $0.4 . "$

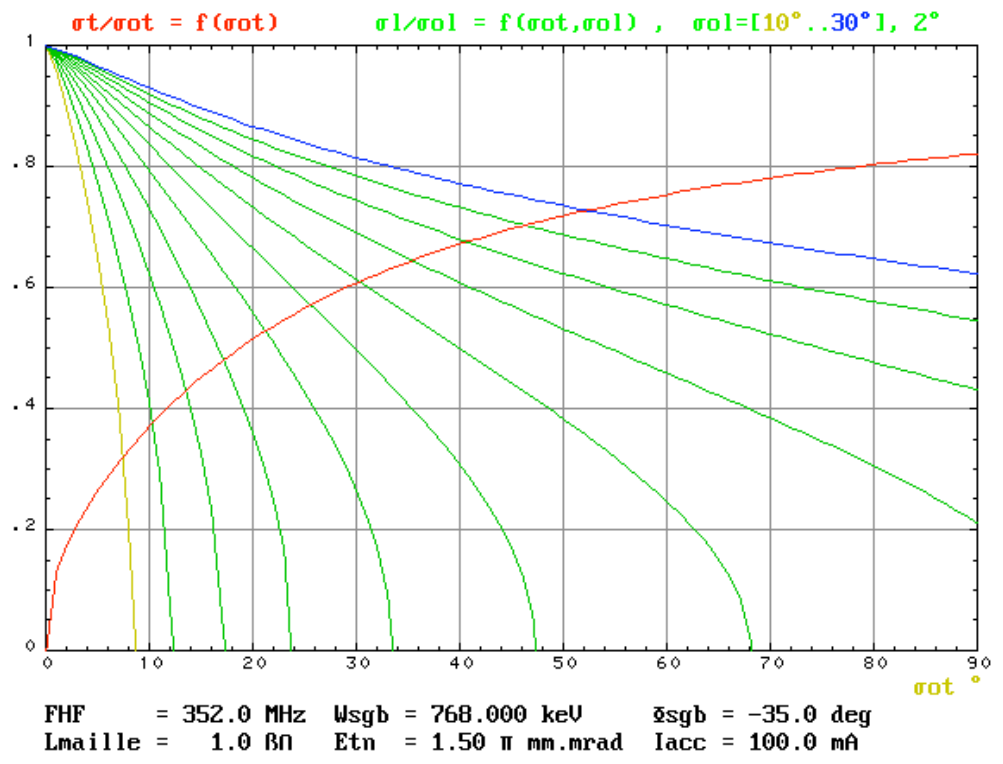

Fig. 4.2 A global design aid - 2. (Caption added.)

(end of direct extraction from [26].)

4) These values were then held constant through the remainder of the RFQ accelerating section.

\subsubsection{An Optimization Approach to the "Conventional" Design}

The paper [27] compares a "constant r0, constant V" design to a "varying r0 and V" design, to "avoid the 'bottleneck' (of the "constant r0,V" design) in the minimum aperture profile at the end of the gentle buncher, where the minimum aperture has to decrease rapidly in order to accommodate the increasing modulation."

Other parameter choice differences between the two designs caused them to have nearly the same length, copper power and transmission characteristics. This was done to show that a lower peak surface field can be used in the "varying r0, V" design for the same length.

It is stated that the "constant $\mathrm{r} 0, \mathrm{~V}$ " design is much easier to machine and tune. In modern design practice, however, the machining and tuning are readily accomplished and would not take any priority over other desirable features.

It was found that beam losses are more localized at the bottleneck in the "constant r0, $\mathrm{V}$ " design. In the "varying $\mathrm{r} 0, \mathrm{~V}$ " design, the minimum aperture decreases slightly through the RFQ and $\mathrm{rO}$ increases according to the modulation; losses more distributed 
along the RFQ, and its transmission is therefore less sensitive to input beam misalignment or increasing transverse input emittance.

Also stated as an important advantage of the "constant r0, V" approach is that it is easier to find an optimized solution in the multi-parameter design space. At this date, the optimization process is still indeed tedious, but advantages of a more encompassing optimization are worth the extra work in the design phase. For example, the beam losses should be confined to occur at the lowest possible energy, in order to minimize buildup of radioactivity. More sophisticated parameter variation methods described below are aimed at including such important aspects in the optimization process.

For an intense, factory-type linac of IFMIF class, all aspects point to design sophistication beyond "constant rO, V."

\subsection{The IFMIF Alternative CDR RFQ Design}

As seen in Sections 2.2 and 2.4, the IFMIF Alternative CDR design has the "conventional" design, with nearly constant voltage and r0 in the main part of the RFQ. The "bottleneck" in the aperture is apparent (Figs. 2.2-1 and 2.4-1). The voltage is higher at the front end, and falls to the end of the shaper section, from which it remains nearly constant except for a slight adjustment at the bottleneck.

For the Alternative CDR RFQ, changes were made in the IFMIF Specifications used for the CDR RFQ. The normalized rms input emittance was raised from 0.2 pi.mm.mrad to 0.25 pi.mm.mrad, reflecting an estimate of how the ion source and LEBT would perform, easing the space-charge forces in the RFQ. The injection energy was lowered from $0.100 \mathrm{MeV}$ to $0.095 \mathrm{MeV}$, which would produce a shorter RFQ if all other conditions remained the same. A KP factor (KP) of 1.8 was used, to have the possibility of more focusing and consequently less space-charge effect than the KP $=1.7 \mathrm{CDR}$ design, and reflecting confidence that this field level is practical.

Another parameter variation is introduced in this design. In practice, the vane tip is machined with a transverse radius Rho. Varying the ratio of Rho/rO changes the ratio of the peak surface field to vanetip field, the capacitance between adjacent vanes, and the size of the multipole potential terms. In the Alternative CDR RFQ, the Rho/rO ratio is varied, to give lower peak surface field (lower KP factor) at the fixed vane voltage in the downstream part of the RFQ (Fig. 3.2-2). As the higher voltage in the front end is not required to achieve adequate focusing, the strategy there could be modified.

The ability to vary $\mathrm{Rho} / \mathrm{rO}$ is an important feature, and could be exploited in the final IFMIF RFQ design.

In Fig. 2.2-1, note that after the bottleneck, in the accelerating section, the modulation and aperture, thus also r0, and synchronous phase are constant. The vane voltage is essentially constant, thus also B. The consequences of this on the beam dynamics, e.g., the longitudinal emittance and beam loss behavior (Fig. 3.2-1) are discussed in Section 6. 


\section{Beam-Based Linear Accelerator Design Technique}

This chapter describes accelerator design techniques that go beyond those commonly used, and which must be understood by project team members, as well as project leaders, reviewers and other interested parties.

Design of an RFQ is the focal point of this discussion, but it applies to linear accelerators of all types.

Repeating from the introduction to Section 4: "Conventional" intense RFQ design, still used almost exclusively today, is exemplified by the design of RFQs as originally laid out by Los Alamos in 1978-1980, in which only a global beam-current-related criterion was invoked for guidance as to the practical maximum current that should be accelerated for given focusing parameters, plus a cell-by-cell recipe for assigning the synchronous phase. The linac is laid out, keeping many parameters constant in the main accelerating section to ease manufacturing and tuning as understood at the time. Then beam acceleration is simulated and the resulting transmission to the output is observed as the measure of successful performance. The detailed pattern of beam loss has rarely been studied. It has been considered "too difficult" to understand in detail the cell-by-cell performance of the beam as it undergoes the very complicated process of forming a bunch from an initially dc beam and accelerating. This method is thus characterized as design "from the external field to the beam result," i.e., "from the outside in."

This method does involve the injected beam in the design, but not the detailed beam behavior inside the RFQ. For intense, factory-type linac facilities like the IFMIF, where continuous operation for a period of $\sim 40$ years is expected, and minimized radioactivity buildup is a key objective, a more rigorous design method was sought.

An inverse design procedure is more relevant to the problem of achieving low beam loss: a beam-based design procedure starting from the space-charge physics characteristics of the desired beam, and finding the external fields appropriate to confine it.

Therefore the author has derived a "from the inside out" beam-based approach, in which the desired space-charge physics of the beam is first specified at each cell, and then accelerator fields are derived for the desired conditions.

The beam-based method requires a practical formulation of the space-charge physics, understanding of the effect of accelerator structure resonances and their spreading by space-charge, phase-space transport mechanisms [28], controlled use of a beam internal energy equilibrium and the parameters related to it, and other factors.

A major requirement of the beam-based method is that the desired design performance be very closely verified by the detailed beam simulation. This was not lightly achieved, and required extensive development of the design method to include all of the effects to be simulated, and of the simulation code itself. These tools are discussed in Section 8 . 
Rules governing the space-charge physics of intense beams in linacs were introduced into the cell-by-cell design procedures starting in 1981 [17]. The evolution of the design methods, and their application to the CDR and Post-CDR RFQ designs presented above, are discussed in the following paragraphs.

\subsection{Extension of the "Conventional" Procedure to Achieve Shorter RFQs}

Through experience, it was quickly found that RFQs designed by the "conventional" procedure tended to be longer than desired. Other trial-and-error procedures were developed that produced shorter RFQs with nearly as good transmission; these methods remained largely private however.

A thorough investigation of the "traditional" gentle-buncher design procedure and the previously trial-and-error extension to shorter RFQs is given in [29]. Finding a satisfactory synchronous phase at the end of the gentle buncher corresponded to keeping the tune depressions there $\geq \sim 0.4$. It was found that the conventional approach was allowing the tune depression to be $<0.4$, and often even to go to zero (the spacecharge limit), within the gentle buncher. The conclusion was that for intense beams, the single-point rule at the end of the gentle buncher was inadequate, and that the tune depression in the gentle buncher should be maintained above zero. For moderate to low beam intensities, the original procedure produces adequate results, although the RFQs are often considered long. In addition, the complication of the parameter space was shown, and how shorter RFQs are produced.

Other improvements were found empirically by experienced designers. [30,31,32,33,34] It was realized that lower focusing at injection eased input matching, and as a consequence that a lower vane voltage can be used in this region, saving $\mathrm{rf}$ power.

It was found that relaxing the transverse focusing in the accelerating section to keep the transverse phase advance more similar to the longitudinal phase advance gave better transmission. The reason for this was made clear by the author in the following amplification of the space-charge physics relations between the accelerator structure and a beam.

\subsection{Space-charge Physics Relations Between the Accelerator Structure and a Beam}

For a rigorous and practical beam-based design technique, the central requirement is to use all available beam physics information in the design process; the resulting design is then checked by a simulation code with the same underlying physics.

\subsubsection{The Beam-Envelope Matching Equations}

In the "conventional" design, the beam-envelope matching equations 1-6, Section 4.2.2, are used to set a "current limit". For a beam-based design, they are invoked at every cell. 
The equations describe the variation of the rms beam radius and length, as a function of the external fields and the internal space-charge forces within the beam, which work to counteract the external fields. They describe a bunched beam of ellipsoidal form, so apply exactly after the beam is bunched enough to be described as an equivalent ellipsoid.

Sacherer [35] showed, in his derivation of these equations, that the actual form of various particle distributions causes only a few percent difference in the solution, if the rms properties of the distributions are the same - a seminal result that enables an "equivalent rms" design method. The remaining difference is, however, important to achievement of the best beam-loss performance. The design strategy incorporated in LINACS can account for the variation in the form factor of the ellipsoid, and this is effective in reducing beam loss ${ }^{1}$.

The equations cannot be extended to account for emittance growth - the derivation requires the emittances to be either constant, or to have a functional form known apriori. The ability to use an apriori form is valuable for certain design problems.

Cylindrical beam envelope equations can also be written. The beam transition in the RFQ from cylindrical to ellipsoidal form is however very complicated and a precise enough (less than $\sim 2 \%$ error) analytical expression for the transition has not been found.

The matched beam radius and length should vary smoothly in order to avoid unwanted effects, so that the derivative of the beam size is allowed to vary only slowly approximately adiabatically in terms of the betatron and synchrotron oscillation wavelengths, so that $\mathrm{a}^{\prime}=\mathrm{da} / \mathrm{dz}$ is $\sim$ zero. When the second derivatives of the beam size are $\sim 0$, the beam is "matched" transversely and longitudinally:

Now in addition to the external field variables contained in the zero-current phase advances, four new variables appear - the transverse and longitudinal rms beam sizes and emittances.

Equations (1) and (2) may be solved for any two variables; the others must be prescribed.

\subsubsection{Beam Equilibrium - The Equipartitioned Condition}

One other space-charge physics relationship that can be employed for design is known, called the equipartitioning relationship $[15,17]$, which requires that the energy content within the beam be equal in the transverse and longitudinal degrees of freedom. When this condition is satisfied, there is no free energy within the beam that is available to drive a resonance condition:

$$
\begin{aligned}
& \frac{\varepsilon_{t n} \sigma_{t}}{\varepsilon_{\ln } \sigma_{l}}=1 \\
& \text { which also implies } \frac{\varepsilon_{l n}}{\varepsilon_{t n}}=\frac{\gamma b}{a}=\frac{\sigma^{t}}{\sigma^{l}}
\end{aligned}
$$

${ }^{1}$ The form factor adjustment is a complex subject and beyond the scope of this report. 
The availability of an equilibrium, or equipartitioned (EP) condition for the beam is of course a powerful advantage. As explained below, the equilibrium requirement can be invoked at will by the designer; its utility varies according to detailed design requirements.

As the emittances can varied apriori, it is seen that the equipartitioned condition can be applied over a wide range of conditions, and can change within the accelerator.

The equilibrium condition can be required in addition to the matched conditions, and Eqs. (1), (2), and (7) solved simultaneously at each cell.

Now there are three equations, which may be solved for any three variables; the others must be prescribed. Typically, the beam transverse and longitudinal radii must be matched, and the EP condition forces a relationship between the beam radii. Thus two of the equations are effectively used for the beam radii, and the third equation to invoke the EP relationship through the use of one of the available RFQ external parameters, for example, the vane modulation.

\subsubsection{Phase Advances - Resonances}

Eqs. (1), (2), and (7) represent a typical nonlinear system, with all the complex behavior $[28,36,37]$ that today receives very much attention in the field called nonlinear dynamics, with which the particle accelerator community is in general not familiar. It is interesting that the field of nonlinear dynamics itself changed significantly in the past decade or so. Previously, it was attempted to explain "complex behavior" or "chaos" using theory stemming from a perturbed Hamiltonian. Some relations were found, for example, the Lyapunov Criterion which indicated whether a system was chaotic, which seemed to be useful even though the system was perturbed very far beyond the infinitesimal perturbation over which the theory was valid. More recently, a geometrically based theory of nonlinear behavior has been developed, which can handle large perturbations, and in which it becomes clear that the mechanism for phase-space transport is resonances - with which the accelerator community is very familiar. A combined point of view is useful [38].

With no beam (zero beam current), the structure resonances are defined completely by the local external fields, at all rational number combinations of the tune ratio $\sigma_{\mathrm{o}}{ }^{l} / \sigma_{\mathrm{o}}{ }^{t}=$ sig01/sig0t. With beam current, these resonances are broadened by the collective effect of space-charge, depending on the $\mathrm{rms} \sigma^{l} / \sigma^{t}=\operatorname{sigl} /$ sigt and the internal tune spread of the beam particles.

All satisfactory RFQ designs should avoid the stronger resonances, either purposely or coincidentally to the design procedure used.

When the beam is in the equilibrium, equipartitioned, condition, there is no free energy available to be converted into changes in the particle distribution through resonant actions. In other words, the structure resonance still exists, but although the beam tunes may be in the resonance band, no action occurs because the beam is in equilibrium and there is no free energy. 
This is the feature of a very useful tune chart for linear accelerators developed by Hofmann [39]. Fig. 5.2.3-1:

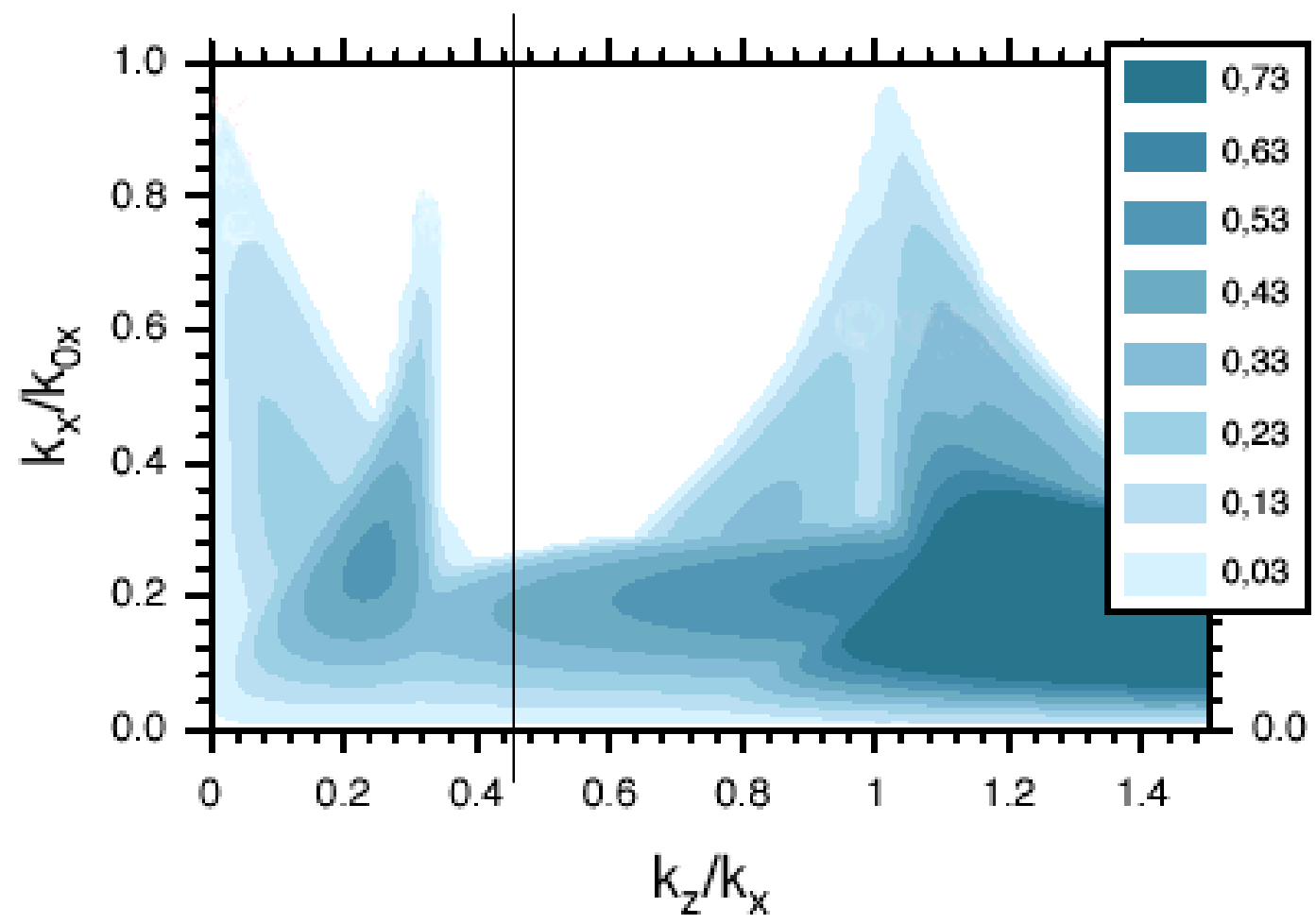

Fig. 5.2.3-1. Hofmann Chart for longitudinal-to-transverse emittance ratio eln/etn $=2.0$; thus $\mathrm{kz} / \mathrm{kx}=0.5$ is the equipartitioned condition for this chart.

The abscissa is the ratio of longitudinal tune to transverse tune. The ordinate is the tune depression, here labeled $\mathrm{kx} / \mathrm{kOx}$ signifying transverse, but in practice, the trajectories of the envelope tunes in the $\mathrm{x}, \mathrm{y}$ average termed transverse, and $\mathrm{z}$ (longitudinal) are both plotted cell-by-cell on the chart. The coloring represents the growth rate of a space-charge broadened tune resonance. Only a few major resonances are shown. On this chart, the growth rates for $\mathrm{kz} / \mathrm{kx}=1,1 / 3$, and smaller fractions are seen. If the emittance ratio remains constant, one chart suffices, but the chart is very dependent on emittance ratio, so if it is changing, several charts must be used.

In the early days of the 1980's, it was very tedious to solve the resonance growth rate equations and only some thresholds for growth were available. However, it was found that if either the transverse or longitudinal tune trajectory went below the threshold, emittance growth in that trajectory would occur [40,41]. Later with Mathematica $\odot$ the charts became practical to generate, and their use is slowly being adopted $[42,43,44]$, albeit mostly to check a design rather than to base a design trajectory on the desired location on the chart, and with some differences; for example, not generally realized are the usefulness of plotting the trajectories in both (or all) degrees of freedom, details about various placements of the trajectory on the chart, or the very detailed evidence of smaller resonances that can be detected. 
As indicated above, resonances are present at every rational ratio of the tunes. In the design process, the major resonances should be either be avoided, or if traversed, then quickly so little growth in emittance occurs, or by requiring the beam to be in equilibrium in the vicinity of the resonance - so that even though the resonance is still there, there is no free energy and it is not excited.

Thus the eln/etn $=2 / 1=2$ chart of Fig. 5.2.3-1 is equipartitioned at $\mathrm{kz} / \mathrm{kx}=1 / 2=0.5$, and this is why no growth occurs in this region, until the tune depression reaches about 0.3 and less.

It is seen that there are areas in the tune chart where the trajectory can be placed without significant growth, especially around the equipartitioned region. It should be remembered, however, that there are an infinite number of smaller resonances. A (nonequipartitioned) trajectory cutting across a region of $\mathrm{kz} / \mathrm{kx}$ that is free from the major resonances shown will still exhibit a small, linearly increasing emittance as the beam is excited by these smaller resonances. The linear growth is typical of such a random scattering effect.

Thus, the EP condition need not be satisfied exactly, and good designs are possible without purposely using the equipartitioning relation. However as will be shown, it is practical and effective to satisfy it nearly exactly after the bunch has been formed. The EP ratios eln $/$ etn $=b / a=\sigma t / \sigma l$ can even be varied along the trajectory if desired, always avoiding growth due to the corresponding resonance. That is, one could purposely traverse a major resonance without significant effect if the beam is maintained in equilibrium during the traverse.

The information represented by the Hofmann chart can also be represented in the typical phase-space plots of nonlinear systems, which show resonance islands, and which change depending on the degree of nonlinearity in the system. As the nonlinearity is increased, resonances begin to overlap. Eventually, the last freestanding resonance is overlapped and the system breaks into complete chaos. The degree of nonlinearity at this point is called the stochastic limit, and is seen to occur on the Hofmann Chart when the tune depression reaches $\sim 0.3$ and below, where there is a general area of growth.

There is a very significant change in the nature of the phase-space dynamics, phasespace transport, and growth rates or disturbance settling times when the stochastic limit is reached. Beyond the stochastic limit, the system is in chaos, with strong mixing and short settling time. In this region, the term temperature can be invoked. Below the stochastic limit, where a linac usually operates, the growth dynamics is completely different, and use of the terms "temperature" and "thermalization" are not appropriate (although rather widely invoked in the accelerator community). The dynamics is characterized as "meta-stable," with very long settling times; there are areas of phasespace that are stable and others that are chaotic. Another way of saying this is that "simplification" by using the space-charge limit (tune depression $=0$ ) is inappropriate for practical design work. 
The Hofmann Chart will be used below to assist in defining and clarifying the beam dynamics of the three sample RFQs. As a reminder, the designer should remember that if the emittance ratio changes, a different chart is needed.

\subsection{Beam-Based Design Procedure}

\subsubsection{LINACSrfq Design Interface}

The ingredients for beam-based design are now in hand - the two matching equations which should always be satisfied, and the third EP equation, which may be invoked if desired and solved simultaneously with the matching equations. Three RFQ parameters, typically the transverse and longitudinal beam sizes, and the modulation if EP is used, are used to satisfy the equations. At this time, the remaining (many) RFQ parameters must be chosen from other perspectives that do not involve beam/structure equations directly. (Design optimization is still an open issue, with many possibilities for more complex expression.)

The LINACSrfq design code incorporates this approach. The designer has cell-by-cell control over all parameters, and over the space-charge physics behavior desired.

It is important to note that now, as the beam itself is specified in terms of its rms emittances as well as current, the design and optimization process are now specific to this beam. This is why certain knowledge of the ion source and LEBT is important. In a factory-type application such as IFMIF, the characteristics of the ion source and LEBT should be reproducible within certain limits (for example, if the ion source is replaced with a spare).

The beam-based design interface for the LINACSrfq code is next outlined to illustrate the design process [7].

\section{(* Set up the general characteristics of the RFQ *)}

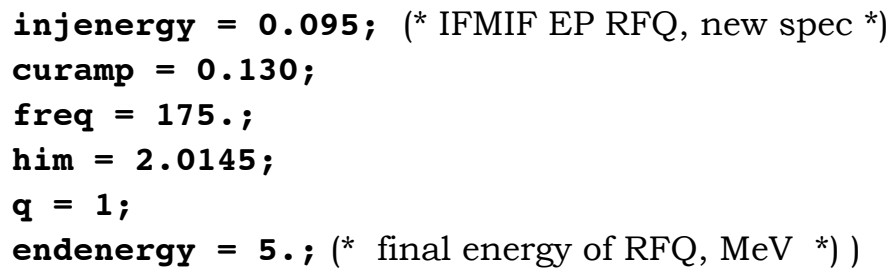

(* IFMIF is a 4-vane type RFQ. Rf power factors are specified - see Section 7 *)

rfqtype $=4$ vane;

powCuLEDAScaletoIFMIF $=7.94$;

powcuFac $=$ powcuLEDAscaletoIFMIF;

(* Initialize V2TERM or VSINE vane geometry functions for 4-vane RFQ. Turn multipoles on (mon) or off (moff), or (mon) with individual control of mpole terms (terms $1=\mathrm{A} 01$ and $3=\mathrm{A} 10$ are always 'on'. mpoleterms(A01, A03, A10, A12, A21, A23, A30, A32 *)

geom $=$ "VSINE"; 


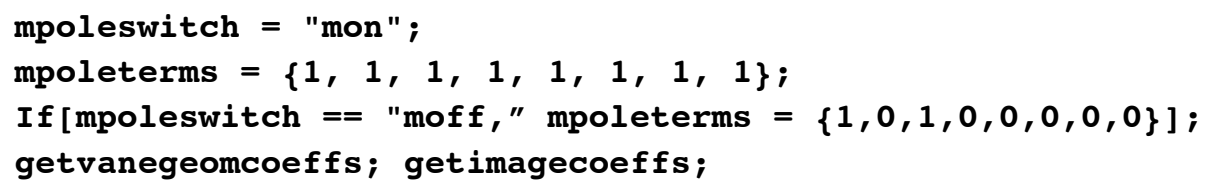

(* Set RhooverR0 (could also be $\mathrm{f}(\mathrm{z})$ *)

RhooverRO = 0.75;

(* The "field" is set to the desired Kilpatrick level KPlimit*KPfac. *)

KPlimit $=$ limkpfld [freq] ; $\quad{ }^{*}=13.98$ at $\left.175 \mathrm{MHz}{ }^{*}\right)$

$\operatorname{KPfac}=1.7 ;$

(* Enter the desired input normalized rms emittances (pi - cm - rad), *) etnrmsgiven

$=0.000025$;

elnrmsgiven $=0.000040$;

(* enter the rms emittances desired in the main RFQ after the shaper: *)

etnrmsgivenmain=etnrmsgiven;

elnrmsgivenmain=elnrmsgiven;

Here are some examples of apriori varying emittance:

$\left({ }^{*}\right.$ etnrmsgivenmain $:=$ If $[(z-z$ start $)<1.0$, etnrmsgiven* $(1-0.2 *(z-z$ start $) / 1.0)$,

$0.000016] ; *)$

(* elnrmsgivenmain := If $\left[z<1.2\right.$, elnrmsgiven, elnrmsgiven $\left.\left.+0.000025^{*}((z-1.2) / 8)\right] ; \quad *\right)$

${ }^{*}$ elnrmsgivenmain $:=-5.3276^{*} 10^{\wedge}(-5)+2.174^{*} 10^{\wedge}(-6)^{*}(\mathrm{i} /$ celldiv $)-1.9926^{*} 10^{\wedge}(-$

$8)^{*}(\mathrm{i} / \text { celldiv })^{\wedge} 2+9.0126^{*} 10^{\wedge}(-11)^{*}(\mathrm{i} / \text { celldiv })^{\wedge} 3-1.9926^{*} 10^{\wedge}(-13)^{*}(\mathrm{i} / \text { celldiv })^{\wedge} 4+$

$\left.1.7246^{*} 10^{\wedge}(-16)^{*}(\mathrm{i} / \text { celldiv })^{\wedge} 5 ; \quad *\right)$

\section{(* Set up the engineering choices and rules for the RFQ *)}

\section{(* Shaper parameters : *)}

The design process starts at the end of the shaper section, where the beam is required to be equipartitioned. The aperture is entered as a fraction of the wavelength, as a comparison to the cell length, which is $\beta \lambda / 2$. Transmission can be optimized by varying this aperture.

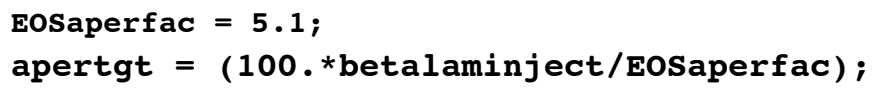

The synchronous phase phis at the end of the shaper is near $-90^{\circ}$, typically -88 to $-84^{\circ}$. The RFQ will be shorter if phis is raised higher, but transmission may be lower.

$$
\text { phistarget }=-84 \cdot \text {; }
$$

Next the reduction in $B$ at the beginning of the shaper is specified:

$$
\text { bfraction }=0.55 \text {; }
$$

Continuing to work backwards, specify the number of cells in the radial matching section.

$$
\text { rmscells }=4 \text {; }
$$


v[rfq] voltage rule for the shaper

frontendvrule: $=($ e.g., the voltage found by the KP limit at the end of the shaper)

Finally, the length of the shaper has to be specified. The program uses a rule involving the integrated zero-current longitudinal phase advance (i.e., the phase advance of a particle very near the synchronous particle), but the relation is not rigorous - siglint is only a number that controls the shaper length. The "porch" is the initial fraction of the shaper length where phis remains at $-90^{\circ}$; phis is raised to phistarget in the remainder of the shaper. The shaper length and porch fraction are used as optimization variables to minimize the energy of lost particles.

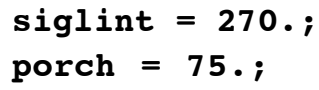

\section{(* Main part of RFQ : *)}

In a final design optimization step, the form factor is adjusted to conform to the actual distribution formed in the RFQ.

(* Interpolating function for ffadj : *)

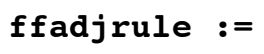

Rules are now specified for the main RFQ parameters. The general form is:

- Specify some rules

- Select the rule to be used in this design

\section{* phis $[\mathrm{rfq}]$ law in main $\left.\mathrm{rfq} .{ }^{*}\right)$}

Specification of the Teplyakov-Kapschinsky Rule, here with saturation at $-20^{\circ}$ if reached. The bucket-beam length ratio can be varied along the RFQ; lfacincr is the change in the ratio, lfacdist is the distance over which the change is made.

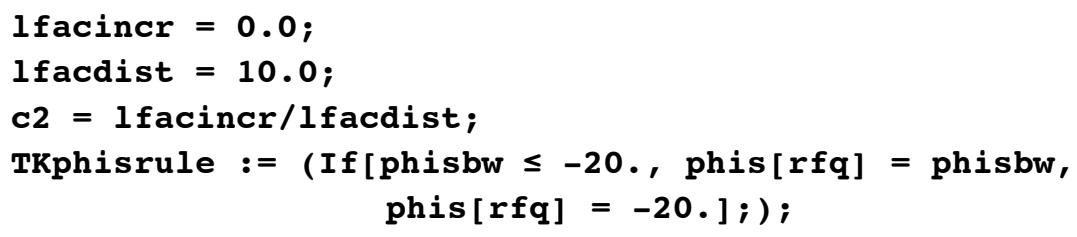

A rule linear with position along the $R F Q$ :

philinear $:=-88 .+43 . *((z-z s t a r t) /(2.7-$ zstart $))$;

Choose which rule to use in this run:

mainrfqphisrule := TKphisrule;

Another example of a rule which could be used: 
(* mainrfqphisrule:=If[philinear $>$ phisbw, phis $[\mathrm{rfq}]=$ philinear, $($ philinear $=-90$;

TKphisrule)]; *)

(*a[rfq] aperture rule for main RFQ : *)

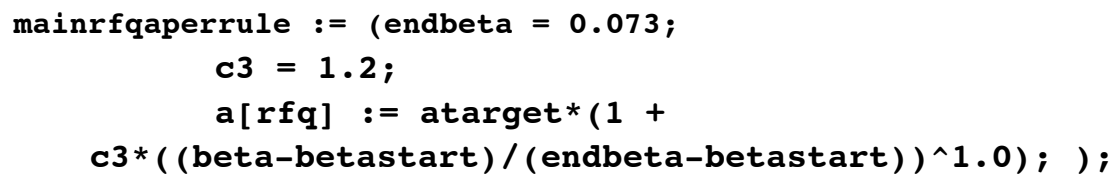

( $\mathrm{v}[\mathrm{rfq}]$ voltage rule for the main RFQ *)

( ${ }^{*}$ mainrfq voltage definitions : *)

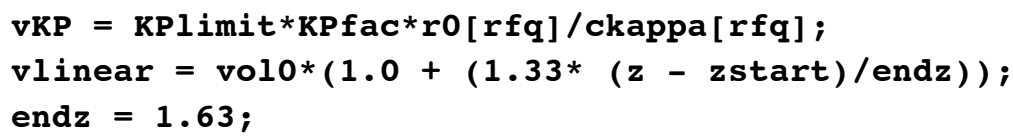

Choose rule:

mainrfqurule :=vKP;

$($ * em[rfa] modulation law in main rfq. *)

$\left({ }^{*} 1\right.$. For EP, em $[\mathrm{rfq}]$ is used to solve for the EP condition. Also used for Constant B strategy. *)

mfree := (em $[r f q]=. ;)$;

(*2. Function for em[rfq] : *)

mfunc : $=($ em $[r f q]=\operatorname{Min}[$ emtarget* $(1 .+1 . *((z-$

zstart) /0.85)^1.0),3.0];);

(* 3. em[rfq] is driven by a cosine function which starts using the em[rfq] slope at the end of the shaper, has length $=$ cossecem, and ends with zero slope at the specified end value of em[rfq](endmodl). *)

cossecem $=1.75$;

endmodl $=3$.;

mCostype := emzz;

(* Set mainrfqemrule to one of the laws *)

mainrfqemrule := mfree;

(* Choose strategy for main RFQ : *)

(* 1. matchonly - will use a rule for em[fq], e.g., mfunc, and the two matching equations.

2. matchEP - em[rfq] is a variable (use mainrfqemrule := mfree;), matching and EP equations are satisfied.

3. matchConstB - em[rfq] is a variable (use mainrfqemrule := mfree;), matching equations are satisfied and $B$ is kept constant.

4. matchboaRatio - em[rfq] is a variable (use mainrfqemrule := mfree;), matching equations are satisfied and $\mathrm{rmsl} / \mathrm{rmsr}$ is kept constant

boaratio $=$ 1.2;

5. matchUser - user written criteria *) 
matchuser $:=$..........;

Select the strategy to be used for this run:

mainRFQstrategy := matchEP;

Everything has now been specified and the design program is ready to run. Many other variations could be specified; all the parameters are under control.

(* Run the subroutines that generate the shaper *)

The front end is generated from the end of the shaper (EOS) backwards. First the EP and matched condition at the EOS is found, then the conditions at the beginning of the shaper, then at the entrance of the RFQ. Working forward, the radial matching section and shaper are generated. The process is iterated using the energy found at the EOS on the first pass. Two passes suffice.

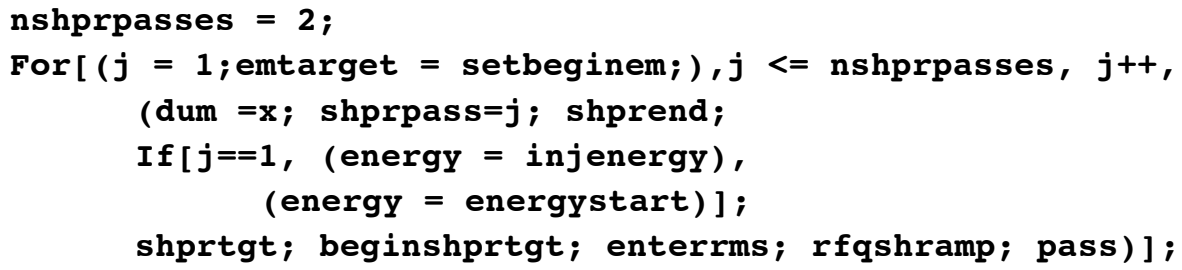

* Run the subroutines that generate the main $\mathrm{RFQ} *$ )

Then the main RFQ is generated from the EOS to the final energy.

phisemrules; rfqaemvar; passmain;

\subsubsection{Design Strategy Discussion}

The RFQ has now three sections - radial matching, shaper (to bring the beam to EP), and acceleration.

Bringing the beam to the EP condition at the end of the shaper (EOS) has proven so robust and useful that it is the standard approach. It is easy to change the shaper rules, for example, to the constant $\mathrm{rO}$ conventional method which exhibits the bottleneck, but tests of this method against the EP at EOS method (ref. Comment in [7]) have consistently shown better beam-loss performance for the EP at EOS method.

Complex rules in the main RFQ can be specified; for example:

- The CDR and Post-CDR RFQs, typical of high-intensity cw RFQs, maintain EP from the EOS to the end of the RFQ.

- Bringing the RFQ to EP at the EOS, and then relaxing the EP condition downstream while taking care to not let the tune depressions become too low, and if a major resonance is crossed, then to cross it quickly, has been an effective method for some applications [44].

Rules are specified for all other parameters.

The original goal of this approach was first to bring all of the RFQ parameters and beam physics (the matching and EP envelope equations, plus some detailed extensions, which are all we know at this time) under complete and flexible control. This is now achieved. The original goal was then to investigate optimization. 


\subsubsection{Design Optimization Discussion}

There are so many parameters, and so many different views of what an optimum constitutes, that optimization if still an open and very interesting research topic. As computer power continues to increase, it becomes more feasible, and modern optimization methods, which can handle many variables and strategies, should be applied to the accelerator design problem.

Experience with LINACS has resulted in an effective, but tedious optimization procedure [7]; after each LINACS step, pteqHI has to be run to check transmission, energy at which losses occur, etc.

1. Get a rough design using the desired design strategy and default values that results in a solution.

2. Find a reasonable input beam ellipse alpha and beta.

3. Optimize transmission varying EOSaperfac.

4. Minimize beam loss above $\sim 10$ times the input energy (e.g., above $1 \mathrm{MeV}$ ) by varying the shaper parameters siglint and porch. The length of the shaper and porch have influence on the transmission and also on the maximum energy of the bulk of the lost particles.

5. Find the input match for best transmission using the transmission matrix option in pteqHI. Using the simulation code pteqHI, the input match must be checked occasionally as the design develops. The most reliable input matching method is to find the transmission over a matrix of input ellipse alphas and betas.

All five of the main RFQ specifications are strongly influenced by the coefficients used in the Teplyakov synchronous phase rule, the voltage and aperture rules, and by the equipartitioning and emittance rules selected.

6. The aperture must open enough as a function of beta to keep radial beam loss low; this raises the voltage and the length.

7. Reduce the RFQ length if possible using a negative lfacincr to allow the beam length to grow relative to the bucket length.

8. Length is also reduced for lower EP ratio. Varying EP strategy may be useful.

9. For lower current designs, abandoning EP after the shaper may be useful.

10. Iterate, iterate. Tedious.

When the design has been optimized, a final optimization step almost always produces another significant improvement in transmission - an adjustment in the design code of the space-charge form factor, sometimes augmented by an apriori adjustment of the transverse and longitudinal rms emittances. 


\section{Comparisons of the Three RFQs in Terms of Beam- based Design}

\subsection{The IFMIF CDR RFQ Design}

The optimized CDR equipartitioned RFQ design and simulation were prepared for the CDA [7] and CDR. The input current was specified as $140 \mathrm{~mA}$, to be sure of accelerating at least $125 \mathrm{~mA}$. The input transverse normalized emittance was specified as $0.20 \mathrm{~mm} . \mathrm{mrad}$, the estimated emittance of the ECR ion source. At that time, multiple and image-charge effects were not included in the design code LINACSrfq or the simulation code pteqHI; simulation using codes available at that time which included multipole and image-charge effects indicated transmission of $\sim 95 \%$.

The design optimization (for minimum beam loss at energies above $\sim 1 \mathrm{MeV}$, short length, low peak field, low rf power, high percentage of accelerated particles) was done using the 2 -term potential description. The design maintains an equipartitioned beam from the end of the shaper to the end of the RFQ, with fixed emittance ratio eln/etn = 2.0. Simulation using the corresponding 2-term potential description showed that the beam is very closely equipartitioned from the end of the shaper (EOS) to the end of the RFQ, as indicated in Fig. 6.1-1 and on the Hofmann Chart, Fig. 6.1-2. Fig. 6.1-3 shows small emittance growth after the end of the shaper.

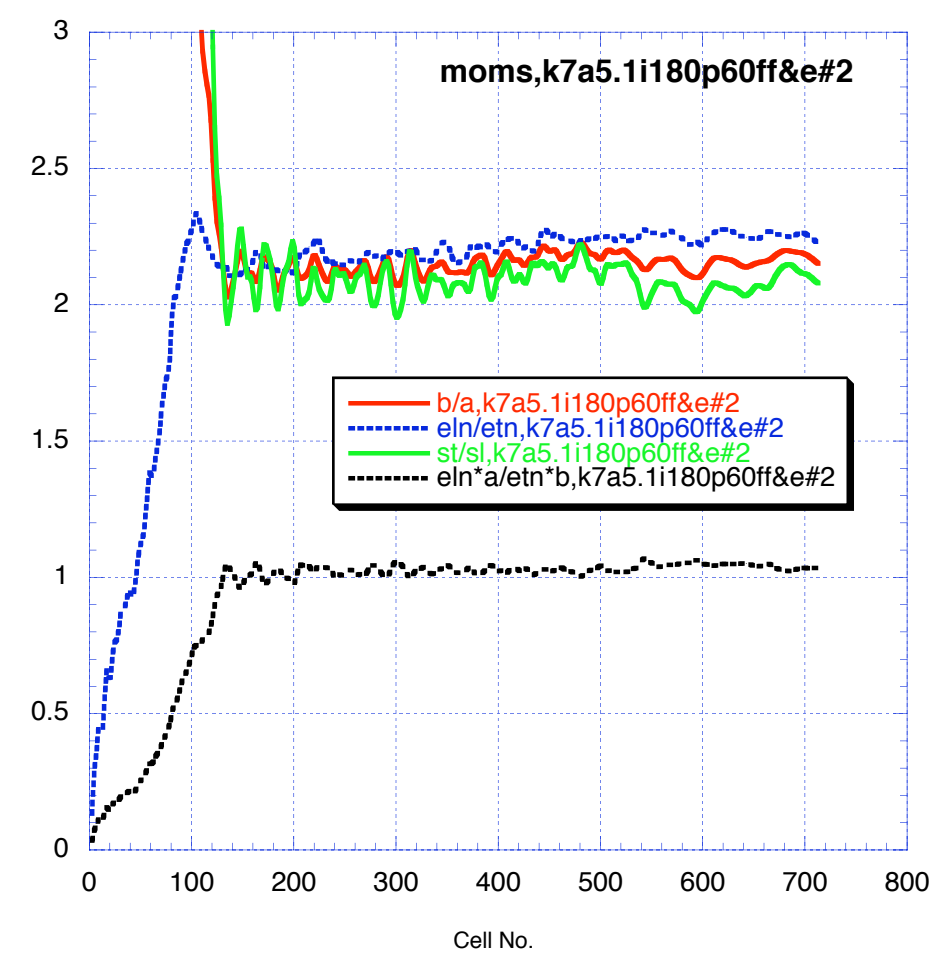

Fig. 6.1-1. Equipartitioning ratio, and corresponding beam size, emittance and tune ratios, Eqs. (7) and (8) for the IFMIF CDR RFQ using the 2-term potential. (Ignore the file ID material after the commas.) 


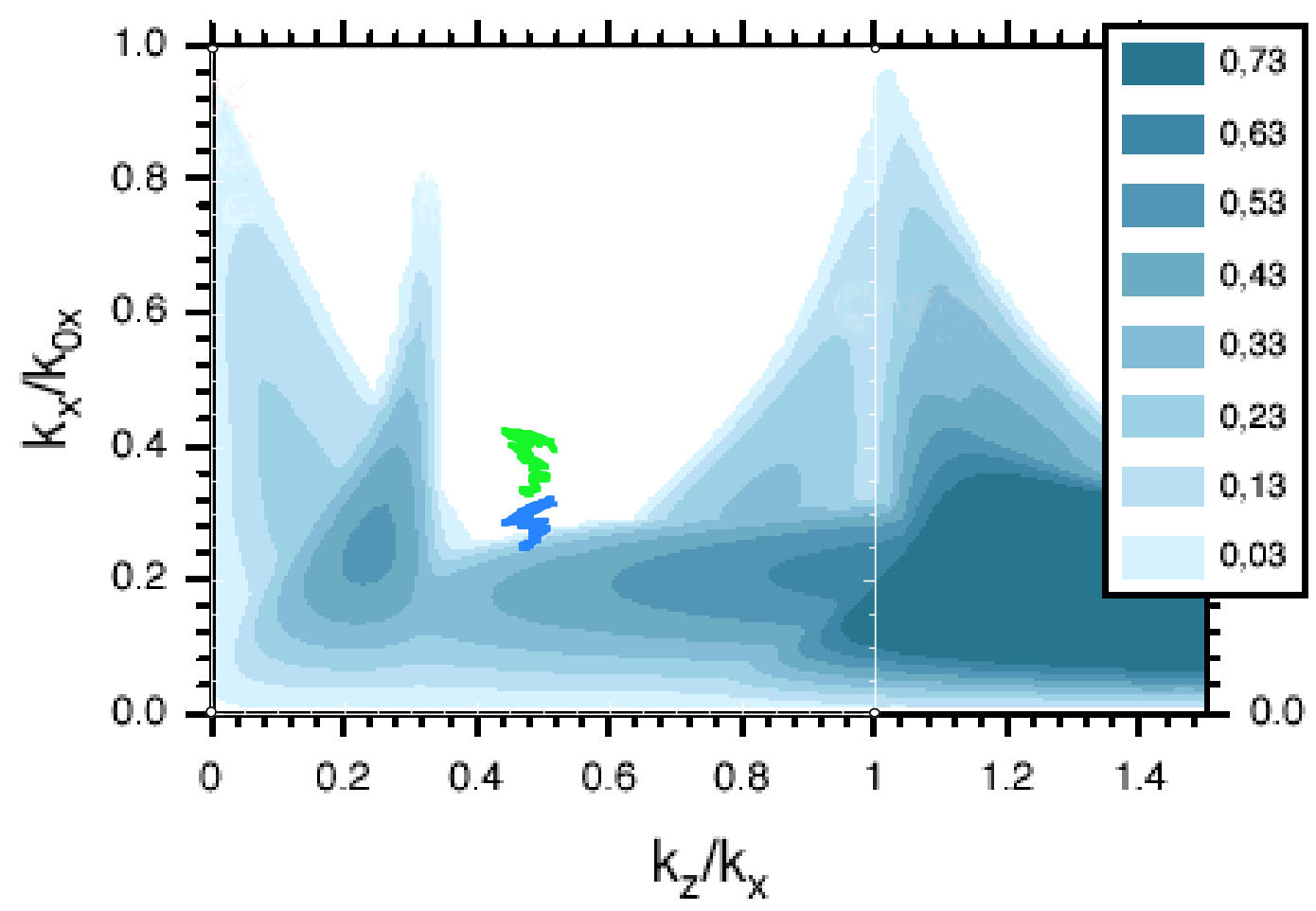

Fig. 6.1-2. Hofmann Chart for eln/etn=2.0, showing the trajectory for the IFMIF CDR RFQ from the EOS to the output, using the 2-term potential.

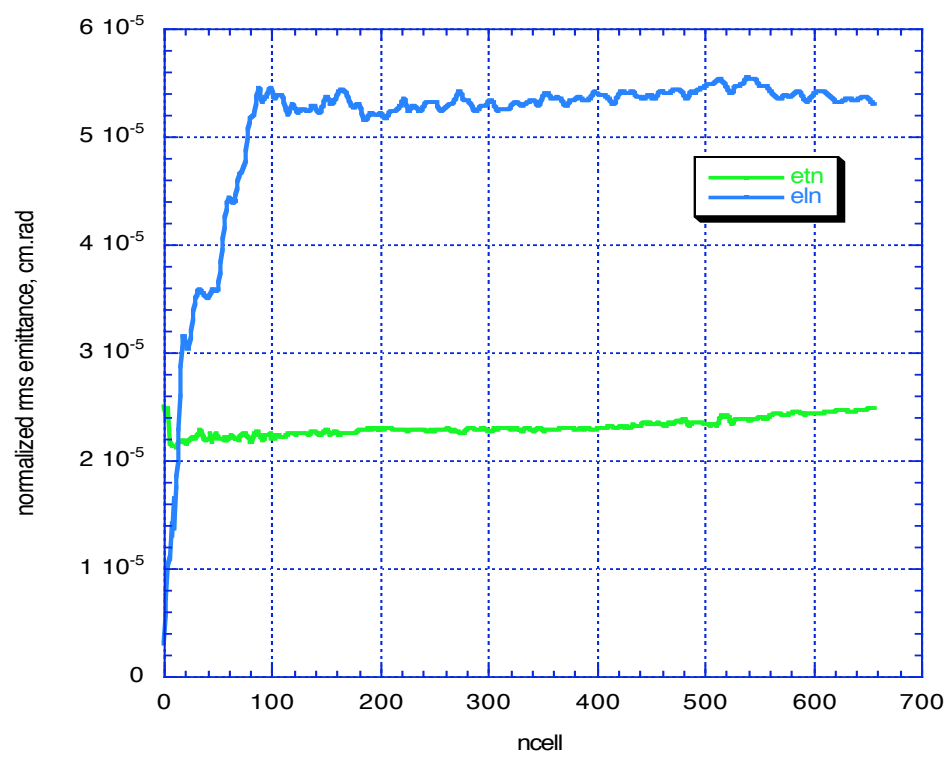

Fig. 6.1-3. Longitudinal and transverse normalized rms emittances through the IFMIF CDR RFQ.

During 2006, multipole and image-charge effects were added to LINACSrfq and pteqHI, and the IFMIF CDR RFQ was resimulated in pteqHI, using the same input beam. 
Section 3 shows results; the accelerated beam transmission drops to $\sim 90 \%$, while the percentage of beam loss above $1 \mathrm{MeV}$ remains low at 0.073\%. Figs. 6.1-4 and 6.1-5 indicate the effect on the space-charge physics; the beam is no longer equipartitioned, and the trajectory has moved toward the resonance to the left. This simulation with multipoles and image-charges is used below in the comparisons.

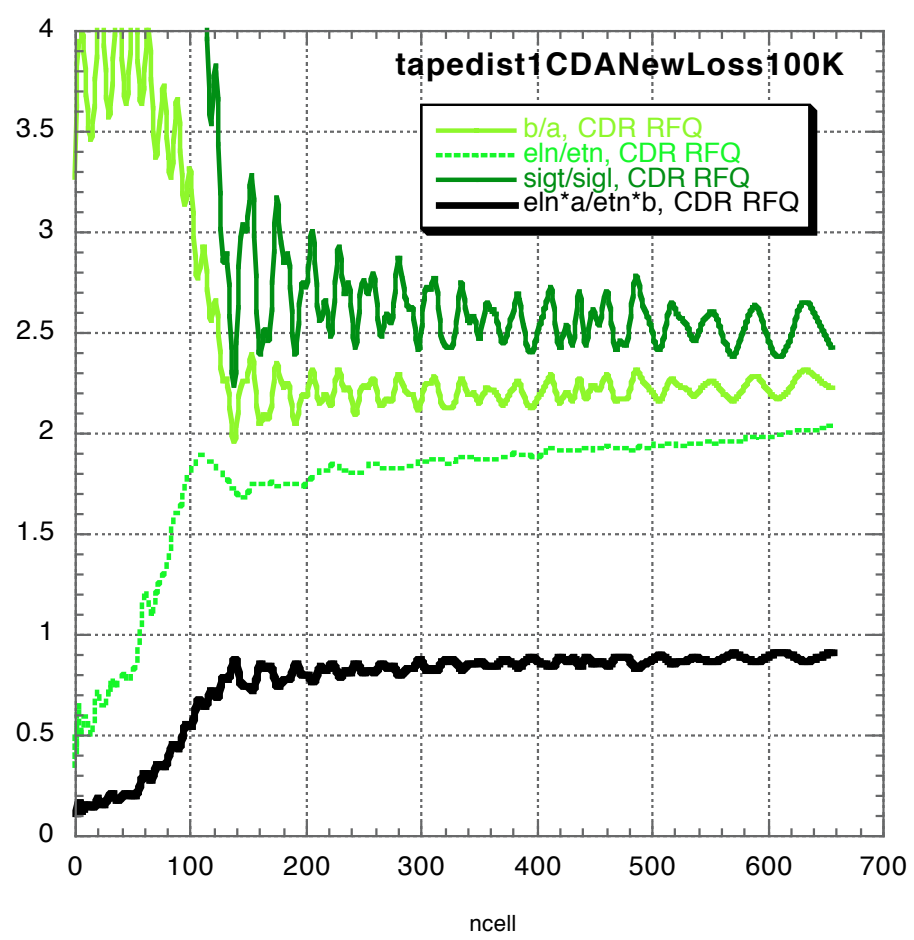

Fig. 6.1-4. Equipartitioning ratio, and corresponding beam size, emittance and tune ratios, Eqs. (7) and (8) for the IFMIF CDR RFQ using pteqHI including multipole and image-charge effects. 


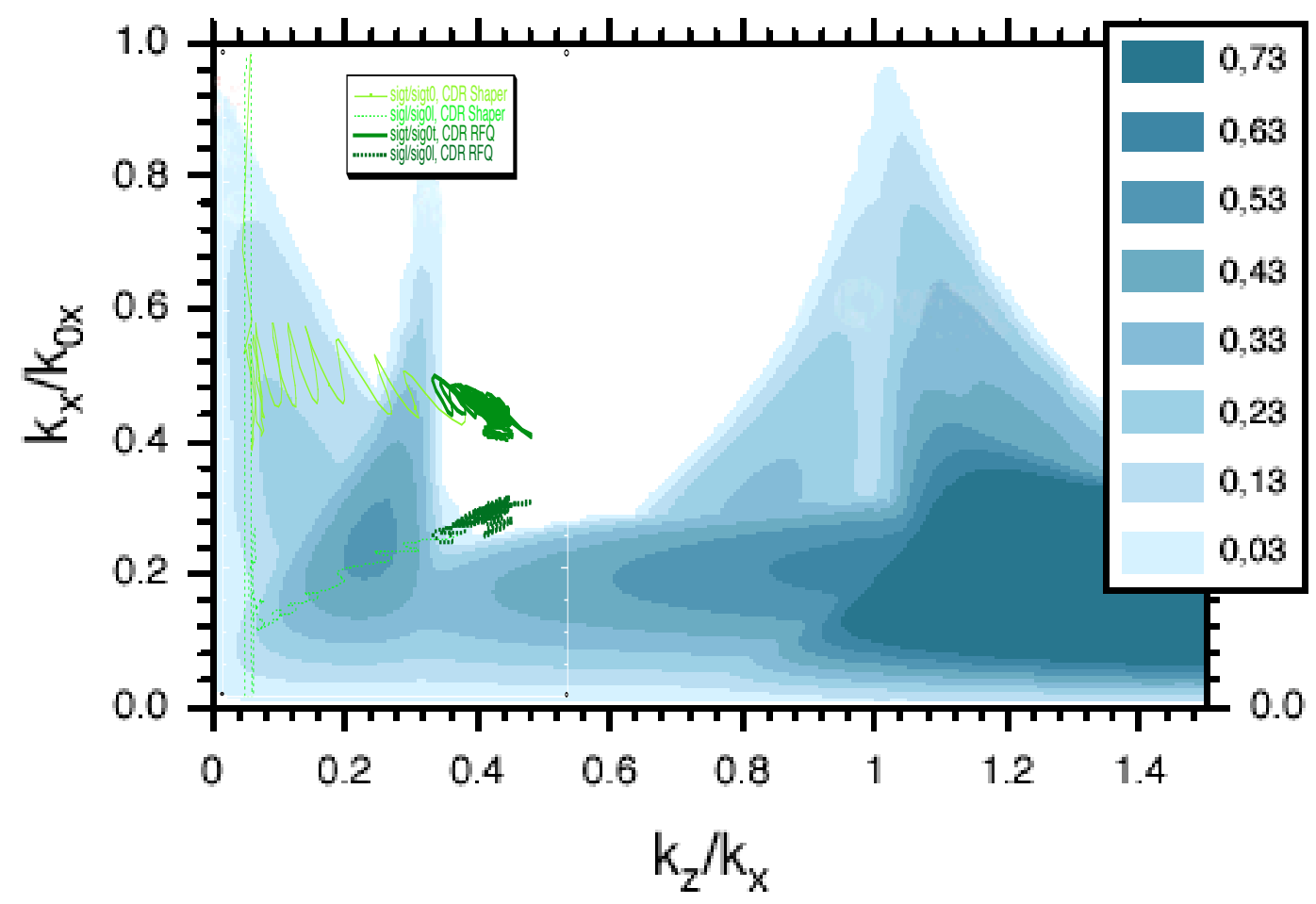

Fig. 6.1-5. Hofmann Chart for eln/etn=2.0, showing the IFMIF CDR RFQ trajectory for the shaper and from the EOS to the output, using pteqHI including multipole and image-charge effects.

The beam size ratio remains nearly constant; the emittance ratio shows growth; Fig. 6.1-6 shows this occurs in the longitudinal $\mathrm{rms}$ emittance. The only difference between Figs. 6.1-1,2,3 and 6.1-4,5,6 is the addition of the multipole and image effects in the pteqHI simulation. The linear growth in the longitudinal emittance is characteristic of a scattering effect. 


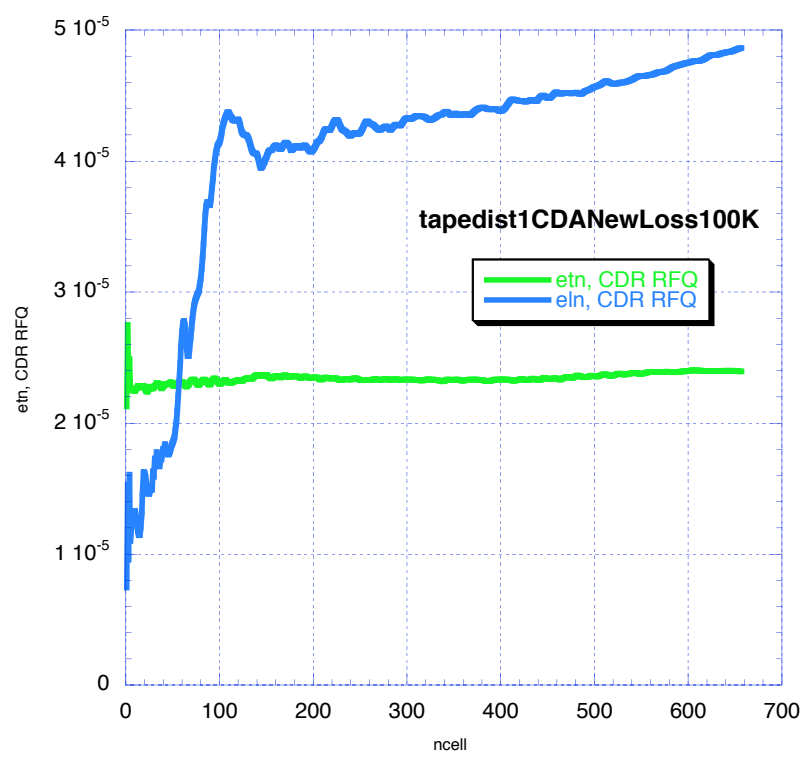

Fig. 6.1-6. Transverse and longitudinal rms normalized emittances for the IFMIF CDR RFQ, using pteqHI including multipole and image-charge effects.

\subsection{The IFMIF Post-CDR Equipartitioned RFQ Design}

\subsubsection{Post-CDR RFQ Design Objectives}

The Post-CDR RFQ design effort has several objectives:

- to determine if the design optimization procedure including multipole and image-charge effects would still function in the same way as the procedure outlined in [7] without multipoles and image-charges. The multipole effects are significant in the beam envelope design code LINACSrfq, while the image-effects are not; both are significant in the simulation code pteqHI. The result was that the optimization procedure remains the same, and the design produces a compensation for the multipole effects, resulting in good transmission and low losses at higher energy.

- to use the recommended lower vane voltage at the front end, for easier matching and lower rf power.

- to explore the trade-off between RFQ length and rf copper power requirement, and the effect on performance. It is seen that good beam-loss and transmission performance can be obtained with shorter length than the CDR design, at approximately the same rf power. It is more important to conserve rf power than to have a short RFQ, because of the rf power operating cost. Very roughly from the CDA Cost Estimate:

CDR Design, requiring $\sim 1 \mathrm{MW}$ rf copper power for the RFQ:

$\mathrm{RFQ}$ structure cost $=($ the average of Acc\#1 and Acc\#2)

$$
=(\$ 15.69 \mathrm{M}+\$ 9.83 \mathrm{M})=\$ 12.8 \mathrm{M}
$$

Accelerator Hall Cost for RFQ $=$ total $^{\star}(5 \mathrm{MeV} / 40 \mathrm{MeV})=\$ 6.7 \mathrm{M}(1 / 8)=\$ 0.84 \mathrm{M}$

Total RFQ Cost $=\$ 13.64 \mathrm{M}$ 
$R F$ system cost $=(\text { total, the average of Acc\# } 1 \text { and } \# 2)^{*}(1 / 8)=(\$ 77 \mathrm{M})(1 / 8)=\$ 9.6 \mathrm{M}$

Rf power bay cost $=\operatorname{total}(1 / 8)=\$ 5.9 \mathrm{M}(1 / 8)=\$ 0.74 \mathrm{M}$

Total RFQ rf power fixed cost $=\$ 10.34 \mathrm{M}$

Total fixed cost for RFQ (no spares) $=\$ 24 \mathrm{M}$

Electricity cost $/ \mathrm{kWhr}=\$ 0.10$

Total electricity cost/year for Acc\# $1=1.33^{*} 10^{\wedge} 8 \mathrm{kWhr} 0.1=\$ 13.3 \mathrm{M} / \mathrm{yr}$

RFQ electricity cost/year $\sim \operatorname{total}(1 / 8)=\$ 1.6625 \mathrm{M} / \mathrm{yr}$

RFQ electricity cost for 20 years $=\$ 33.2 \mathrm{M}$

RFQ electricity cost for 40 years $=\$ 66.4 \mathrm{M}$

Post-CDR Design, requiring $\sim 1.0 \mathrm{MW}$ rf copper power for the RFQ:

RFQ structure cost $=\$ 12.8 \mathrm{M}(8 \mathrm{~m} / 12 \mathrm{~m})=\$ 8.5 \mathrm{M}$

Accelerator Hall Cost for RFQ $=\$ 0.84 \mathrm{M}(8 \mathrm{~m} / 12 \mathrm{~m})=\$ 0.56 \mathrm{M}$

Total RFQ Cost $=\$ 9.06 \mathrm{M}$

Total RFQ rf power fixed cost $=$ same $=\$ 10.34 \mathrm{M}$

Total fixed cost for RFQ (no spares) $=\$ 20 \mathrm{M}$

Electricity cost $=$ same

This suggests that the PostCDR RFQ saves the project $\sim \$ 4 \mathrm{M}$. On the other hand, if the $\mathrm{RFQ}$ length remained at $12 \mathrm{~m}$ and the rf power could be reduced to $\sim 800 \mathrm{~kW}$, a savings of nearly $\$ 10 \mathrm{M}$ would result. Little experience has been obtained so far with optimization; it is possible that a search in the latter direction could be fruitful.

\subsubsection{Post-CDR Specification Changes}

The Post-CDR specifications were changed in accordance with specification changes adopted for the Alternative CDR RFQ:

- the current was reduced from $140 \mathrm{~mA}$ to $130 \mathrm{~mA}$, in expectation that a higher percentage of accelerated current could be obtained from the RFQ to give $125 \mathrm{~mA}$ output current.

- the injection energy was lowered from $0.100 \mathrm{MeV}$ to $0.095 \mathrm{MeV}$. This would result in a shorter RFQ assuming all other conditions were the same.

- the normalized rms input emittance was raised from 0.2 pi.mm.mrad to 0.25 pi.mm.mrad, reflecting a newer estimate of how the ion source and LEBT would perform. This lowers the space-charge effect.

However, the KP factor $=1.8$ used for the alternative CDR RFQ was lowered to KP $=1.7$, the same as used in the CDR RFQ. It was found that the higher KP factor is not needed to provide adequate focusing, and requires more $\mathrm{rf}$ power.

\subsubsection{Intermediate Fixed EP Ratio Design}

An initial optimization attempt explored a lower EP ratios setting, as this has a strong effect on the RFQ length. Figs. 6.2-1,2,3 show the space-charge physics and emittance behavior of an RFQ designed for a fixed emittance ratio $=$ eln $/$ etn $=1.6$, and optimized including the multipole effects. 


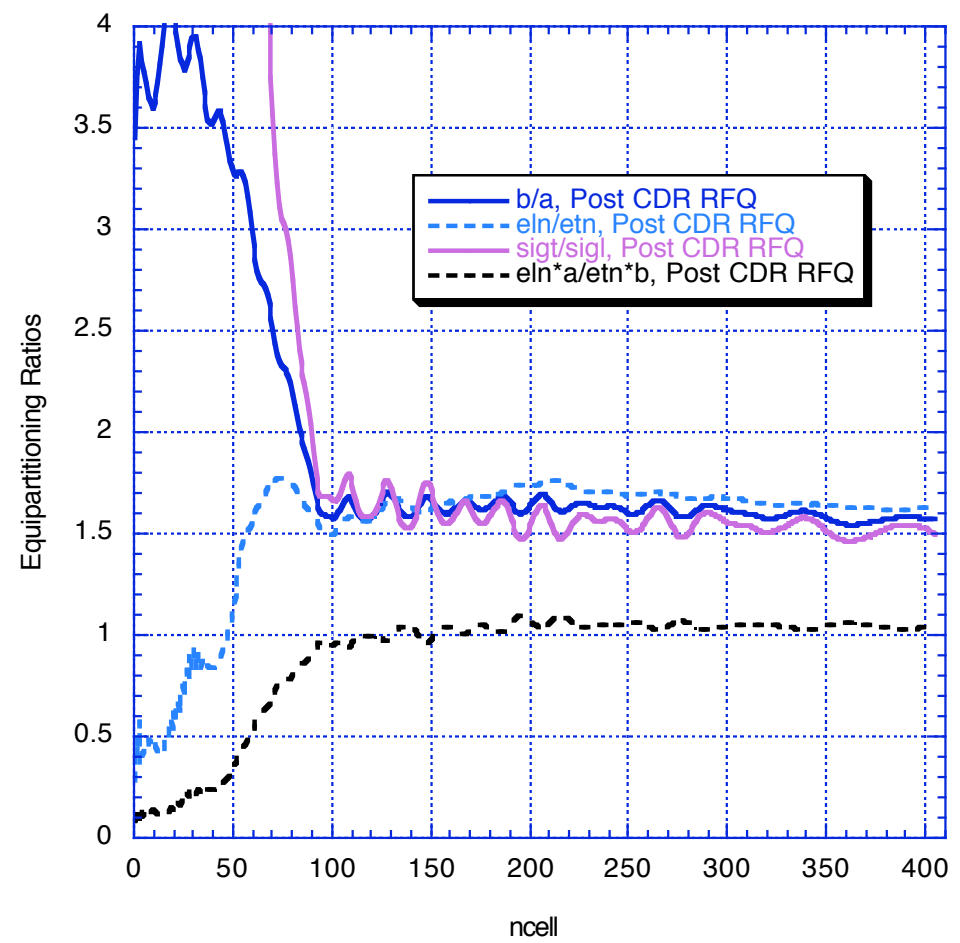

Fig. 6.2-1. Equipartitioning ratio, and corresponding beam size, emittance and tune ratios, Eqs. (7) and (8) for the intermediate RFQ using pteqHI including multipole and image-charge effects.

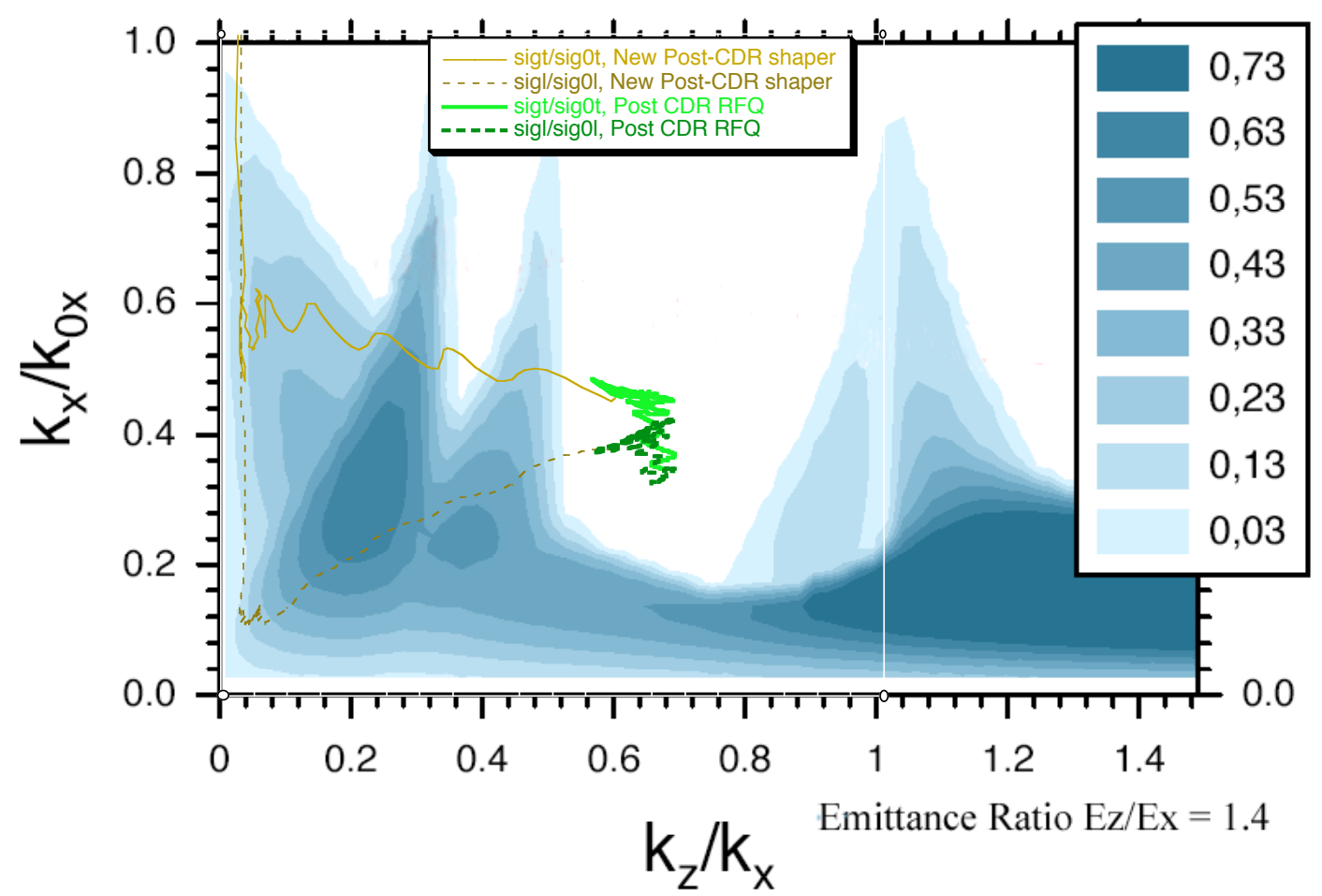

Fig. 6.2-2. Hofmann Chart for eln/etn=1.4, showing the intermediate RFQ trajectory for the shaper and from the EOS to the output, using pteqHI including multipole and image-charge effects. 


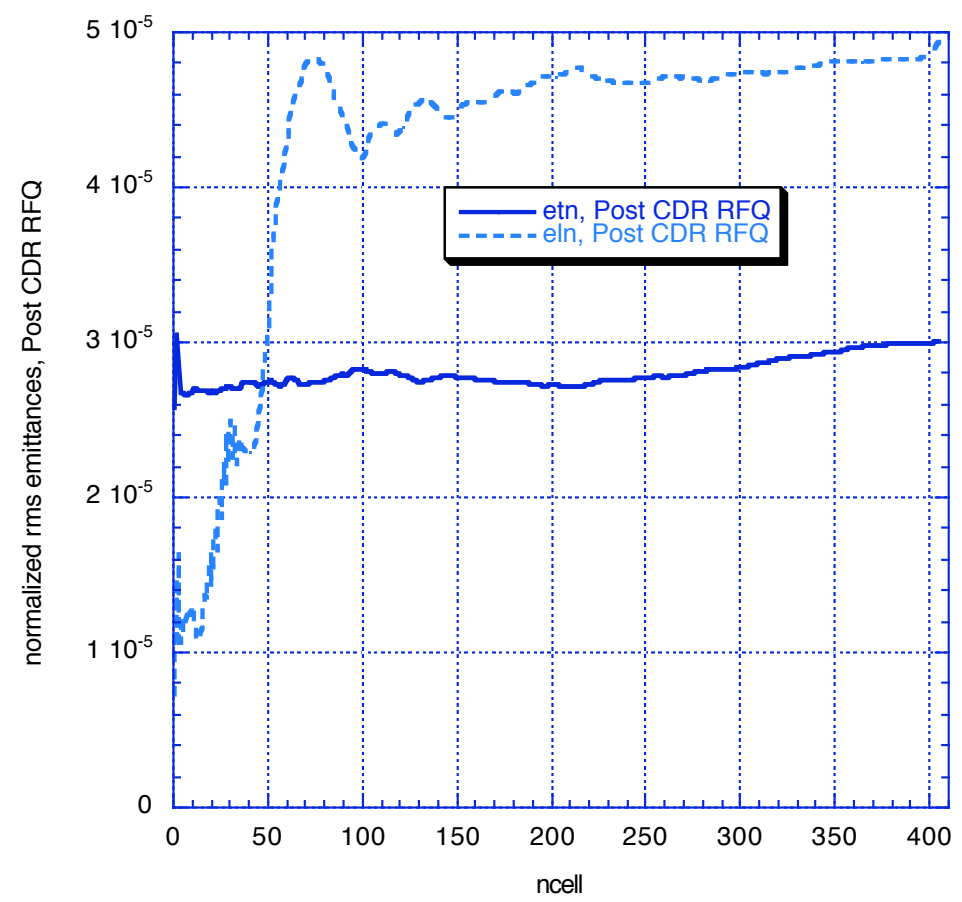

Fig. 6.2-3. Transverse and longitudinal rms normalized emittances for the intermediate RFQ, using pteqHI including multipole and image-charge effects.

The equilbrium, equipartitioned, condition is held closely from the end of the shaper to the end of the RFQ.

This $1.7 \mathrm{KP}$ RFQ requires $\sim 1.0 \mathrm{MW}$ rf power and is $\sim 7.6 \mathrm{~m}$ long, considerably shorter than the CDR RFQ. However, $0.72 \%$ losses above $1 \mathrm{MeV}$ occur for a $1 \mathrm{M}$ particle ideal waterbag input distribution, and $1.15 \%$ for the ion source distribution - 10 times the CDR RFQ, and unacceptable. A large fraction of these losses occurs during the synchronous phase rise after the shaper, and is not influenced greatly by the shaper length and porch.

In these RFQs, the Teplyakov synchronous phase rule is relaxed to let the ratio of bucket-to-beam length reduce somewhat through the main RFQ. This provides a significant reduction in the RFQ length.

Figs. 2.4-12, 6.1-5, and 6.2-3 show longitudinal emittance growth approximately linear with cell number after the end of the shaper. The observed emittance growth suggested a strategy for reducing the losses above $\sim 1 \mathrm{MeV}$.

\subsubsection{Post-CDR RFQ Varying EP Ratio Design}

In the final Post-CDR design, the longitudinal normalized rms eln is given, on purpose, an apriori growth proportional to beta, while maintaining equipartitioning, with the ratios increasing from 1.6 at the end of the shaper toward 2.0 at the end of the RFQ.

The aperture was allowed to grow (also proportional to beta) more than in the intermediate design, to reduce radial losses. This results in a longer RFQ, but it was 
found that the bucket-to-beam length ratio could be reduced more than in the intermediate RFQ, again reducing the length.

With these changes in the main RFQ, the effect of the shaper became more pronounced. Reoptimization resulted in a reduced $\mathrm{B}$ at the beginning of the shaper, and a shorter shaper (with the same fraction of porch).

The resulting KP 1.7, Post-CDR RFQ has $~ 0.081 \%$ beam loss above $1 \mathrm{MeV}$ and $95.8 \%$ accelerated beam fraction for the source emittance input distribution. It is $\sim 8.0 \mathrm{~m}$ long, not including an output Crandall transition cell or output radial matching section. It requires $\sim 1.1 \mathrm{MW}$ rf power, about the same as the CDR RFQ.

Figs. 6.2.-4,5,6 show the space-charge physics and emittance behavior. The ratios rise as a function of beta, and the beam remains closely equipartitioned. A composite Hofmann Chart with eln/etn $=2$ overlaid on eln/etn $=1.4$ is shown, to convey the required change in the EP ratios from 1.6 at EOS to 2 at the end of the RFQ. There is no resonance growth.

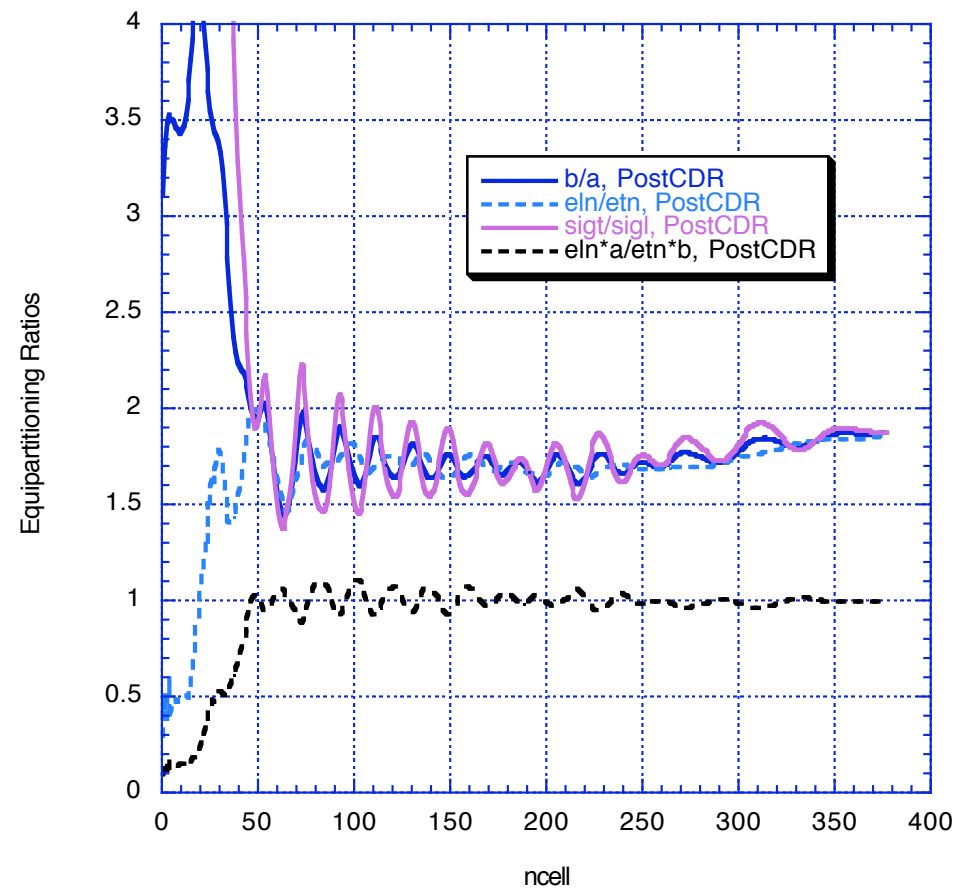

Fig. 6.2-4. Equipartitioning ratio, and corresponding beam size, emittance and tune ratios, Eqs. (7) and (8) for the Post-CDR equipartitioned RFQ using pteqHI including multipole and image-charge effects. 


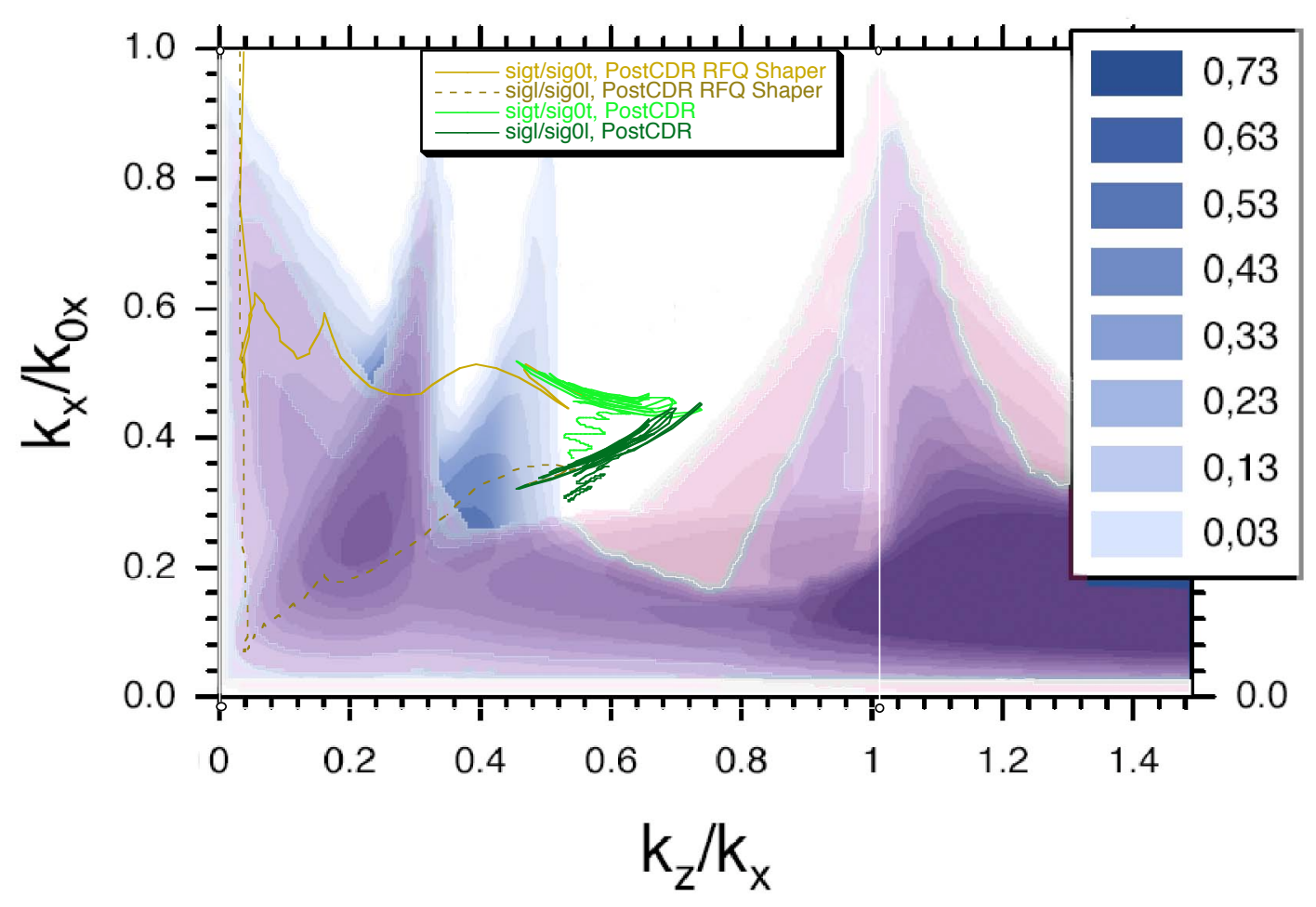

Fig. 6.2-5. Composite Hofmann Chart for eln/etn=1.4 (underlying blue-toned shadows) and eln/etn $=2$ (overlying magenta toned shadows). The Post-CDR equipartitioned RFQ trajectories for the shaper and from the EOS to the output are shown, using pteqHI including multipole and image-charge effects.

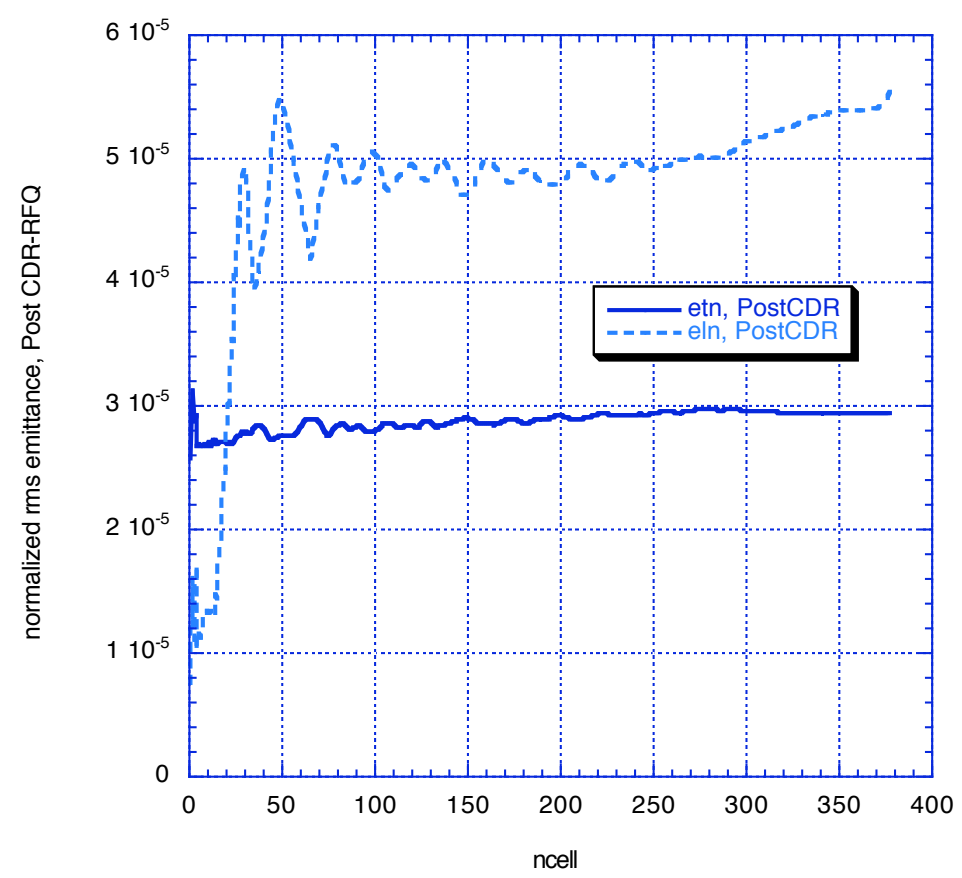

Fig. 6.2-6. Transverse and longitudinal rms normalized emittances for the Post-CDR equipartitioned RFQ, using pteqHI including multipole and image-charge effects. 


\subsection{The IFMIF Alternative CDR RFQ Design}

The space-charge physics of the Alternative CDR RFQ conventional design is more complicated. Figs. 6.3-1,2,3 explain the situation and concerns such as expressed in Section 4.4 .

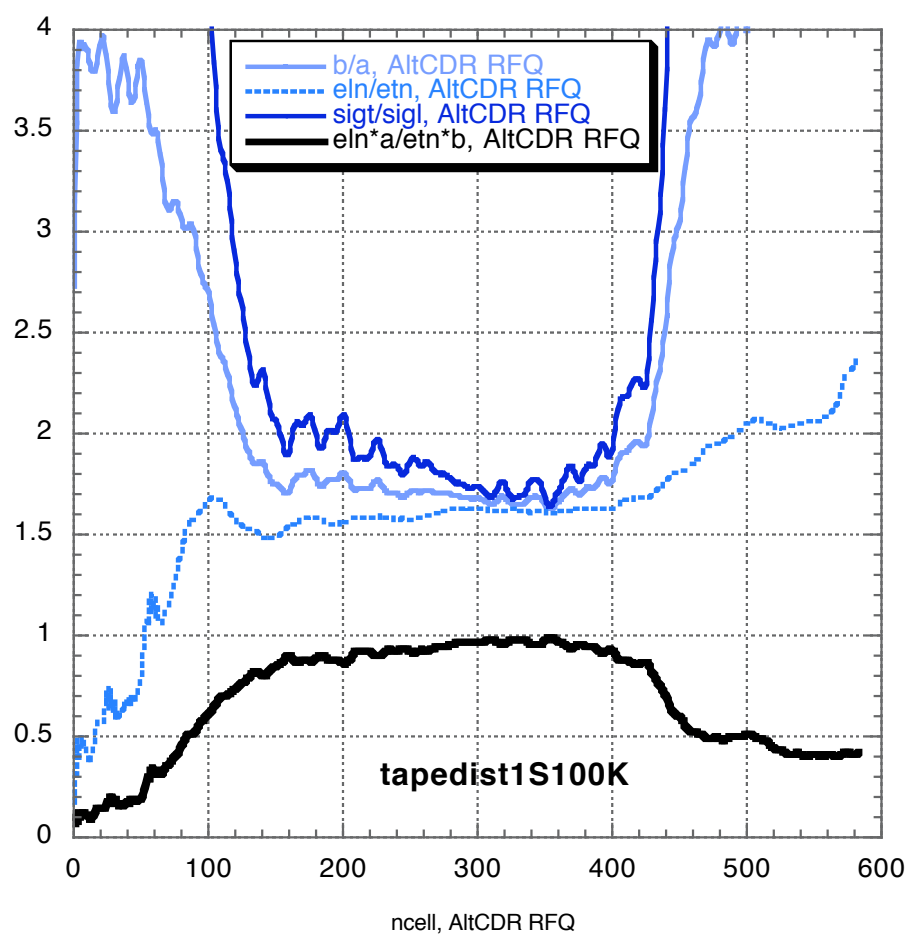

Fig. 6.3-1. Equipartitioning ratio, and corresponding beam size, emittance and tune ratios, Eqs. (7) and (8) for the IFMIF Alternative CDR RFQ using pteqHI including multipole and image-charge effects. 


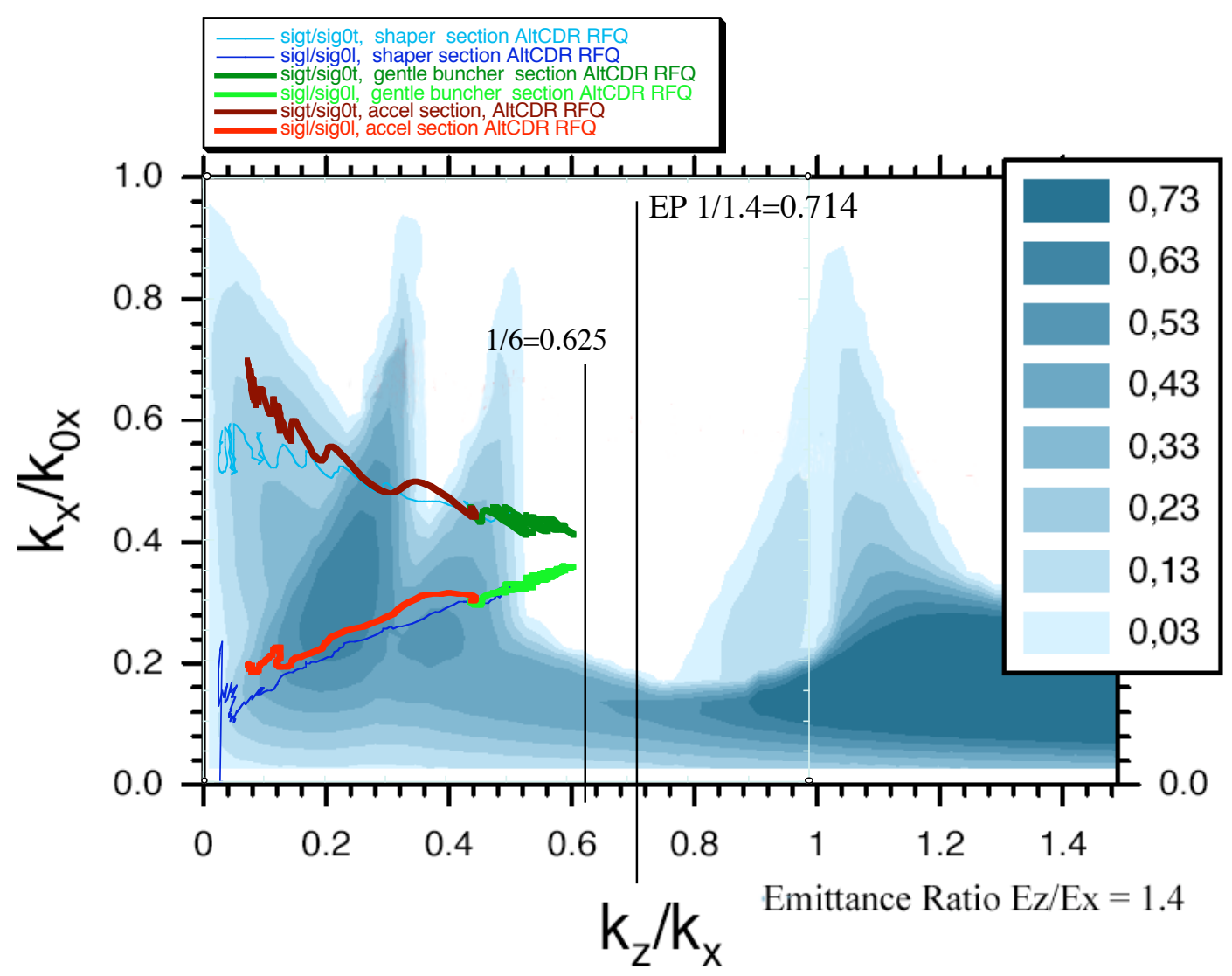

Fig.6.3-2. Hofmann chart for the AltCDR RFQ. Chart is for emittance ratio of 1.4. Beam reached equipartitioned equilibrium briefly at emittance ratio of 1.6 (Fig.6.31). (On a chart with 1.6 emittance ratio, the resonance at $\mathrm{kz} / \mathrm{kx}=0.5$ would be weakened, and the resonance to the left of $\mathrm{kz} / \mathrm{kx}=1$ would be stronger). 


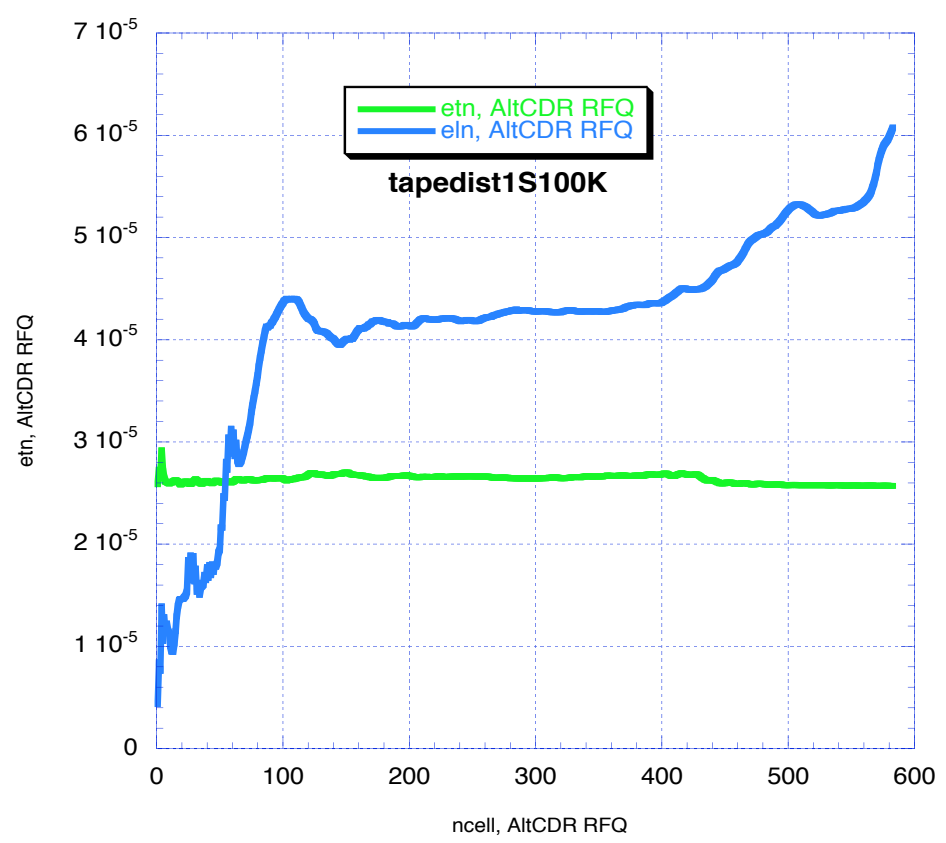

Fig. 6.3-3. Transverse and longitudinal rms normalized emittances for the IFMIF Alternative CDR RFQ, using pteqHI including multipole and image-charge effects.

Near the end of the gentle buncher, the beam coincidentally becomes nearly equipartitioned at emittance ratio eln/etn 1.6. As indicated in Fig.6.3-2, this places the trajectory in a resonance-free region, and the emittances remain essentially constant.

In the acceleration section, the beam departs strongly from EP and there is free energy available to drive resonances. The transverse rms emittance stays essentially constant, with a slight reduction after the end of gentle buncher bottleneck, reflecting the loss of particles in this region. This is a consequence of the conventional design strategy, which maintains strong transverse focusing in the acceleration section. Fig. 6.3-2 shows that the transverse trajectory tune shift rises from $\sim 0.4$ to 0.7 in the acceleration section, and although it traverses the $1 / 2$ and $1 / 3$ resonances, the tune depression is not so low (strong transverse focusing), it crosses quickly, and no transverse emittance growth results.

On the other hand, the longitudinal tune shift decreases from 0.3 to 0.2 , and traverses a strongly resonant region, with directly correlated strong longitudinal rms emittance growth (Fig. 6.3-3) and beam loss (Fig. 3.2-1 and 6.7-2).

Figs. 6.3-4a,4b show evidence of the longitudinal resonances in the $z-z$ ' phase space. The scalloping at the front edge is characteristic of a strong resonance. The tail emerges from the top of the bucket. Fig. 6.3-5 shows the z-z' phase space at Cell 420 , before the onset of the resonances. 

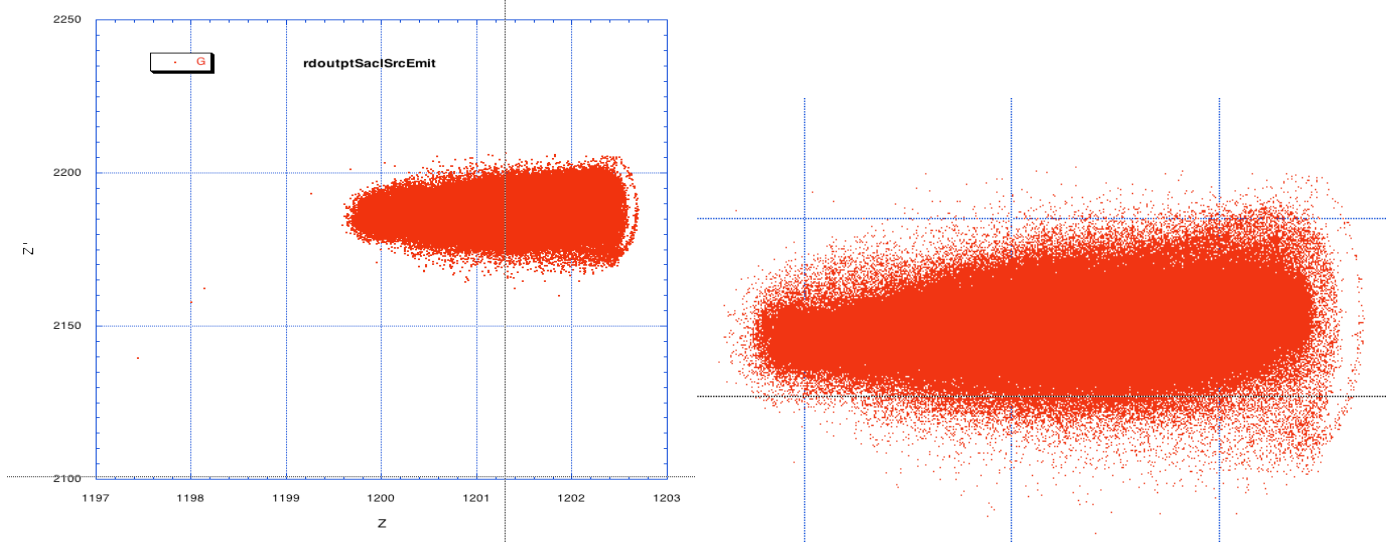

Fig. 6.3-4a. AltCDR RFQ z-z' phase space at the end of the RFQ; 4b - expanded scale

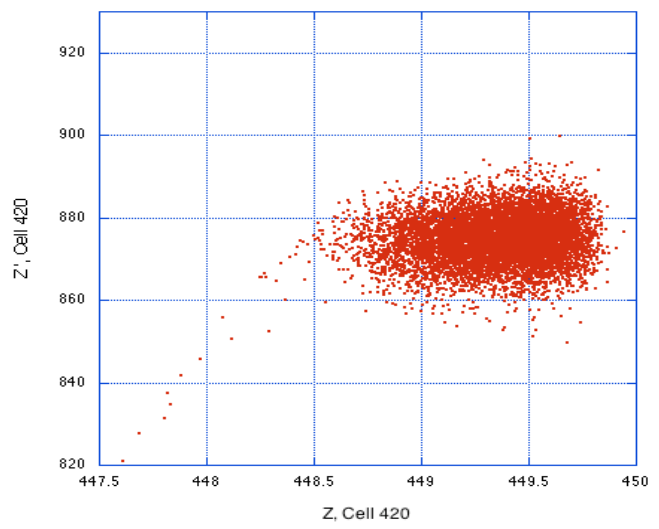

Fig. 6.3-5. AltCDR RFQ z-z’ phase space at Cell 420.

\subsection{Beam Size and Emittance Comparison}

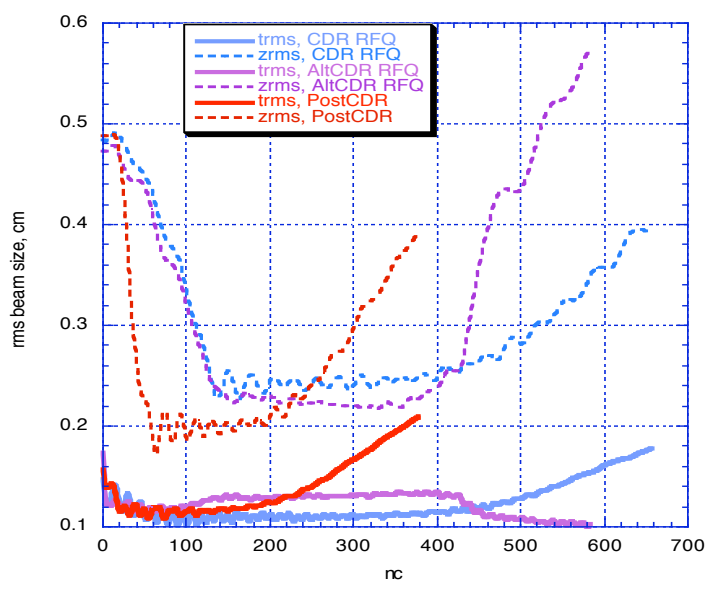

Fig. 6.4-1. Rms beam radius (trms) and length (zrms), $\mathrm{cm}$. 


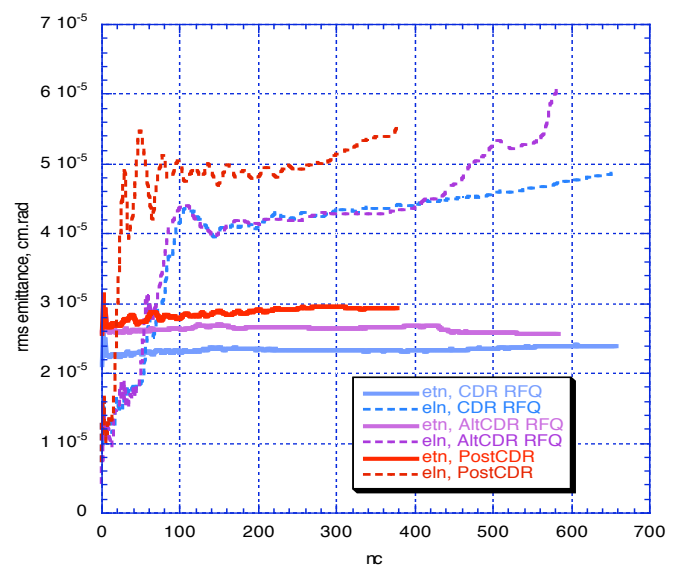

Fig. 6.4-2. Rms normalized transverse (etn) and longitudinal (eln) emittances, cm.rad. The AltCDR and CDR/PostCDR eln are not qualitatively similar, as discussed above. The transverse input distribution is an ideal waterbag, $100 \mathrm{~K}$ particles, etn $=0.25 \mathrm{~mm}$.mrad for each RFQ; an immediate redistribution in the transverse emittance occurs as the distribution adapts to the RFQ.

\subsection{Tune Comparisons}

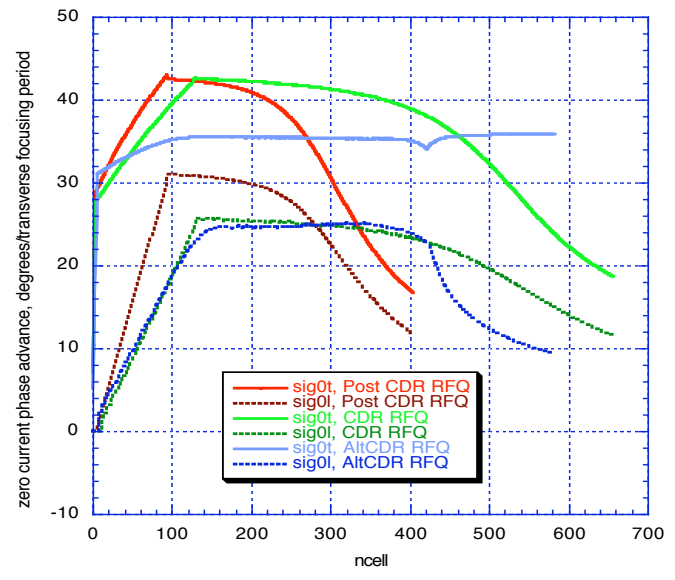

Fig. 6.5-1. Zero-current phase advances/transverse focusing period, degrees. 

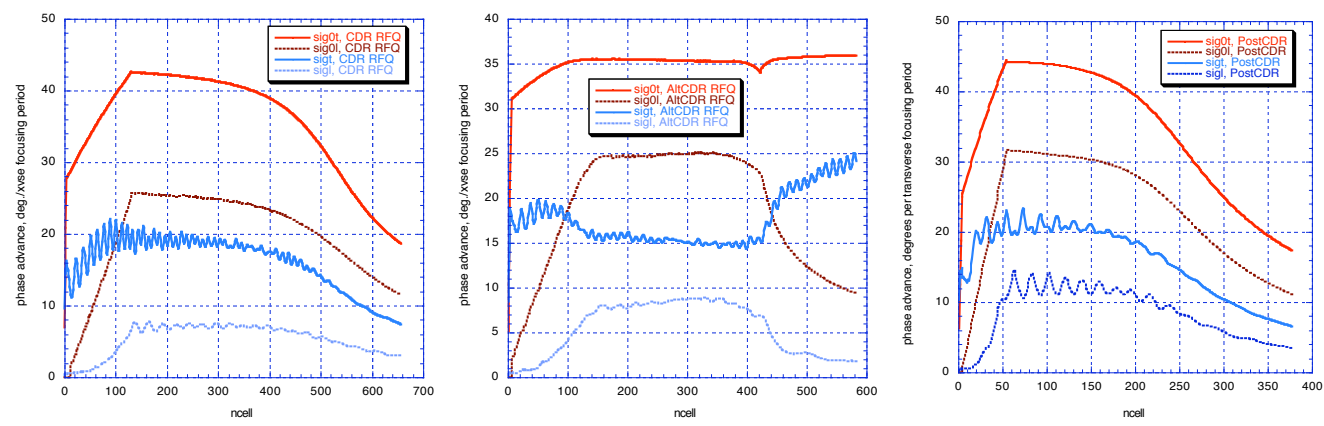

Fig. 6.5-2. Zero-current phase advances $\sigma_{0}$, and depressed tunes $\sigma / \sigma_{0}$, for the three RFQs.
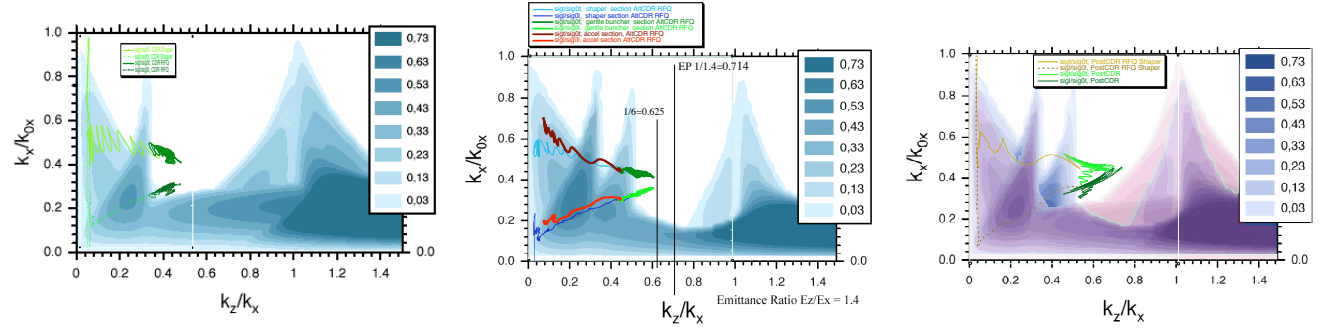

Fig. 6.5-3 Side-by-side comparison of the Hofmann Charts for the three RFQs.

\subsection{Beam-Loss Characteristics}

6.6.1 Representative Post-CDR and CDR Equipartitioned-Type RFQ PhaseSpace Plots and Beam Loss Distribution Characteristics

Plots are at the middle of the last cell. Black: 1M particle ideal waterbag; Red: 1M particle ion source distribution rms matched to the RFQ.

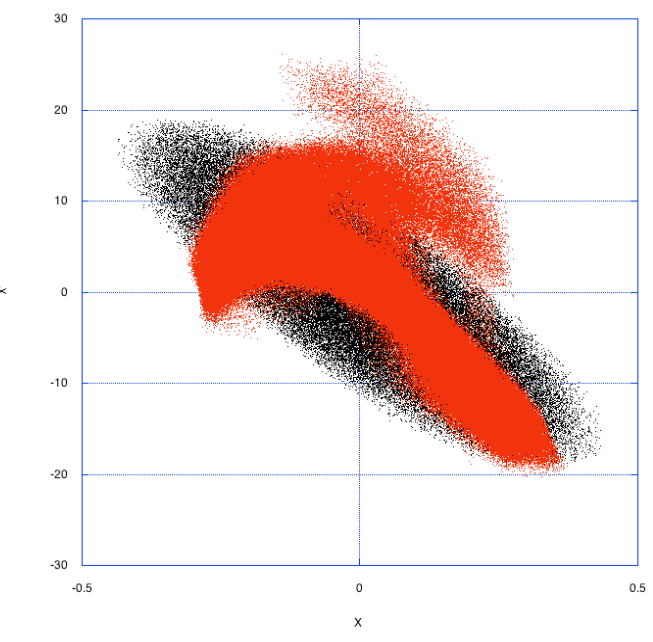

Fig. 6.6-1. RFQ x-x' input distributions.

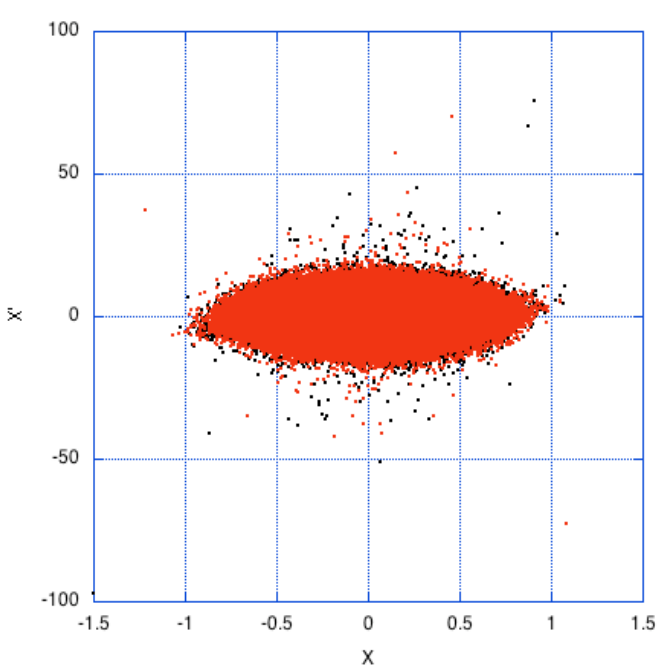

Fig. 6.6-2. RFQ x-x' output distribution. 


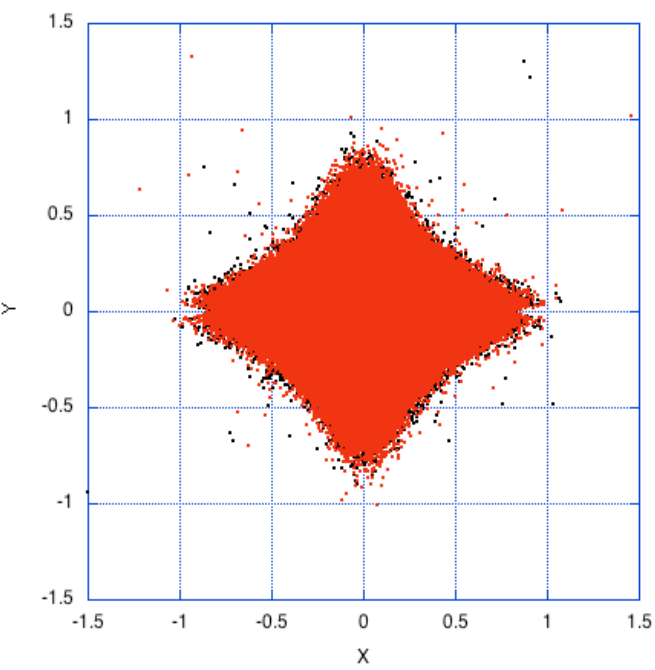

Fig. 6.6-3. RFQ x-y output distribution.

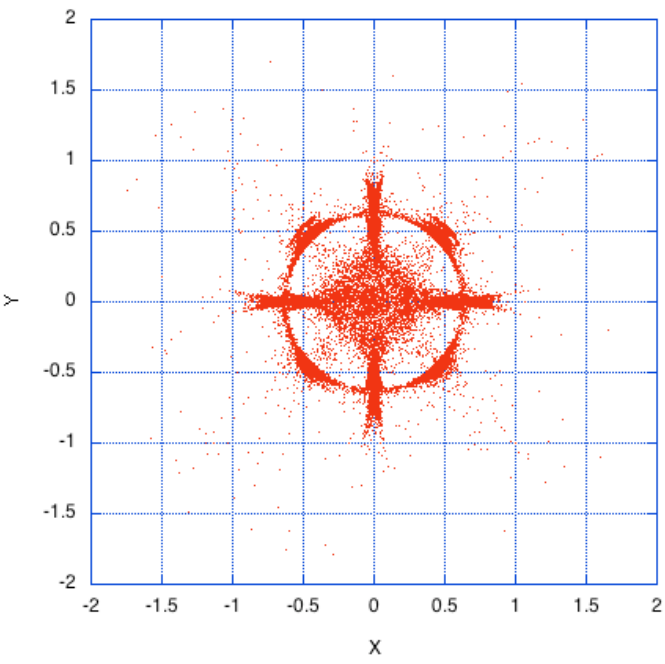

Fig. 6.6-5. $x-y$ distribution of all lost particles.

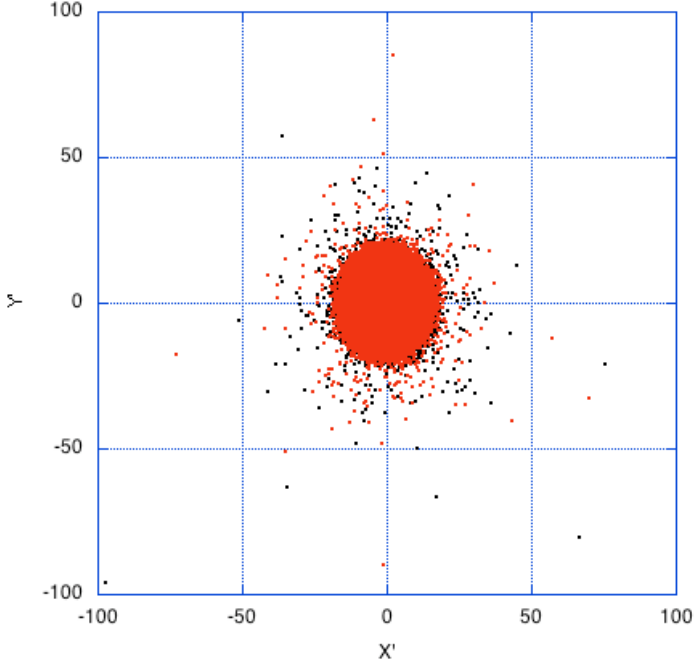

Fig. 6.6-4. RFQ x'-y' output distribution.

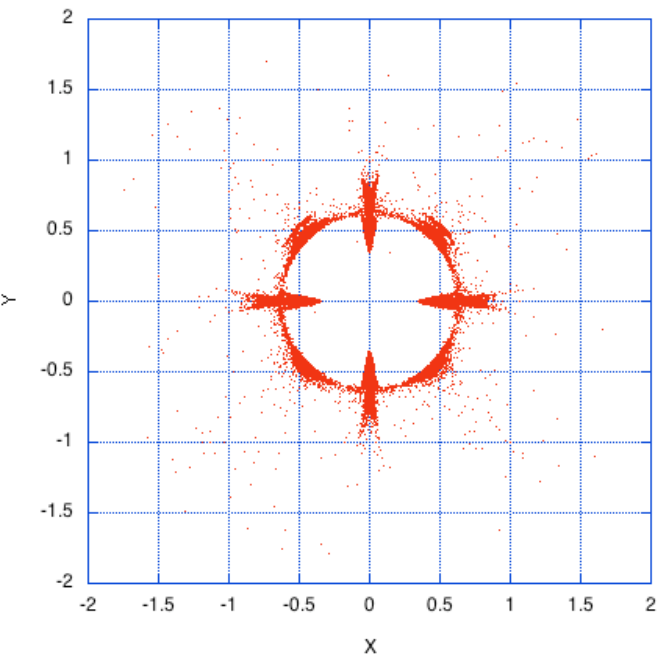

Fig. 6.6-6. $x-y$ distribution of radially lost particles. 


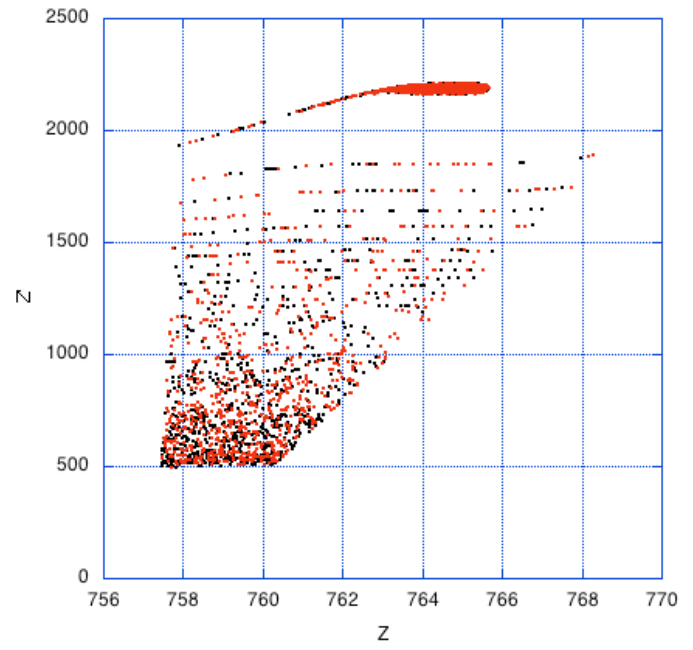

Fig. 6.6-7. RFQ z-z' output distribution.

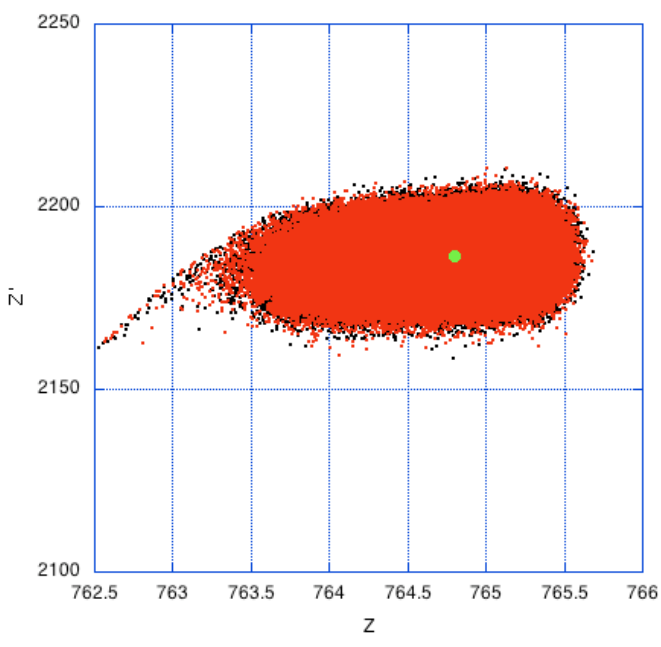

Fig. 6.6-8. RFQ z-z' accelerating bucket output distribution.
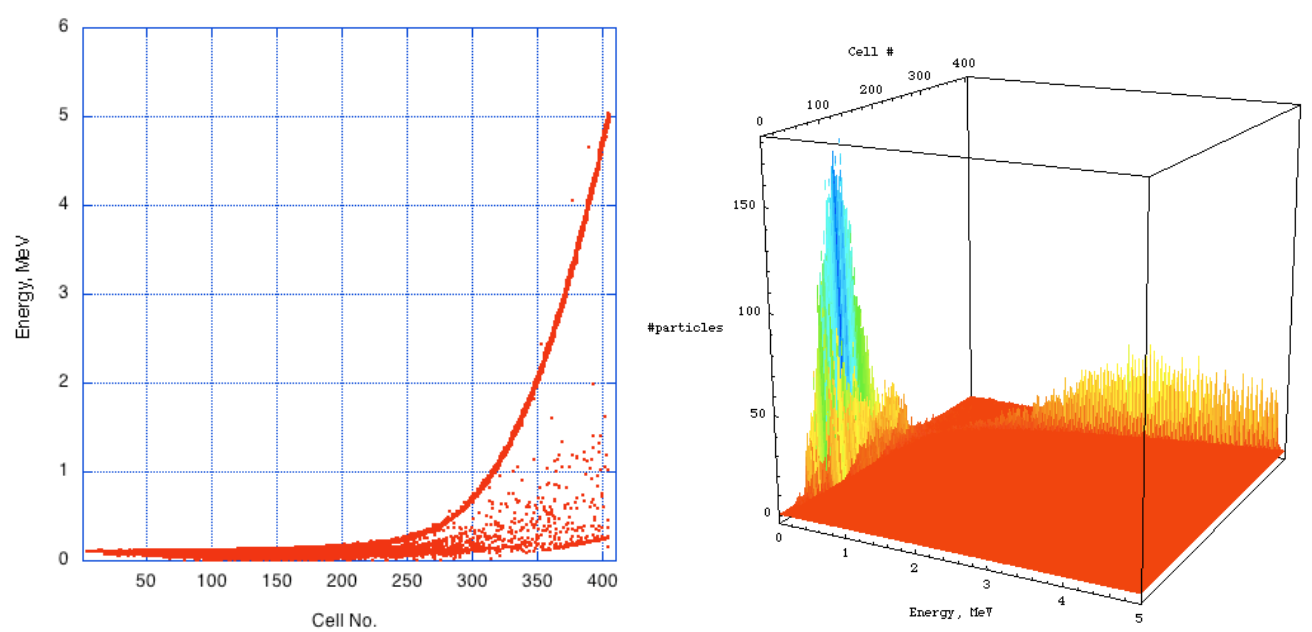

Fig. 6.6-9a,b. RFQ, with ion source input. Particle energy of all losses at each cell. There is a spectrum of lost particle energies at each cell, from $\sim 0.05^{*}$ (synchronous energy) to $\sim$ (synchronous energy). 


\subsection{Beam-Loss Pattern Comparisons}
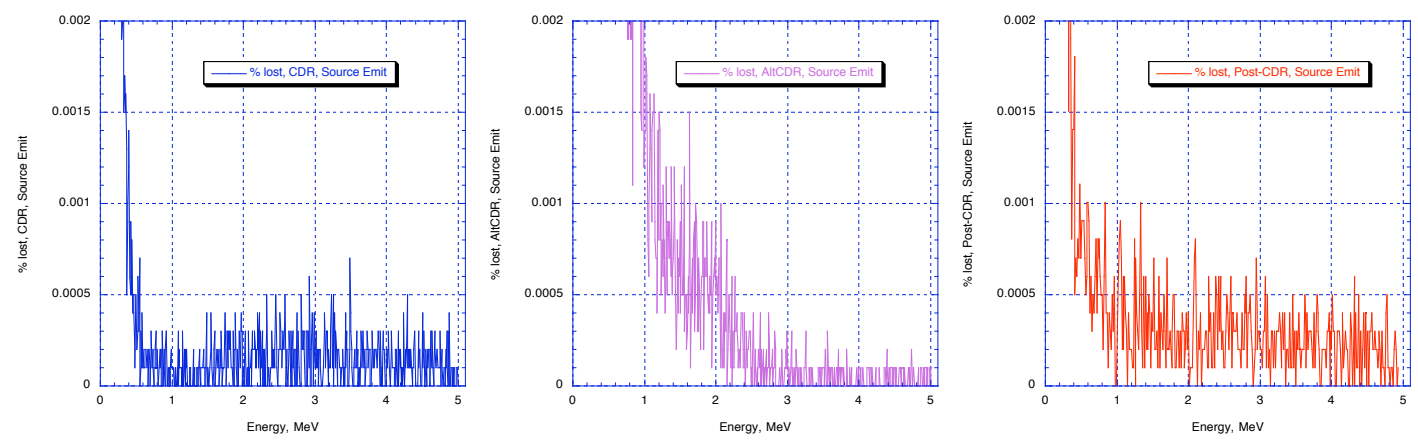

Fig. 6.7-1. Fig. 3.1-3 repeated; beam loss for $\sim 1 \mathrm{M}$ particle ion source emittance distribution, expanded scale.

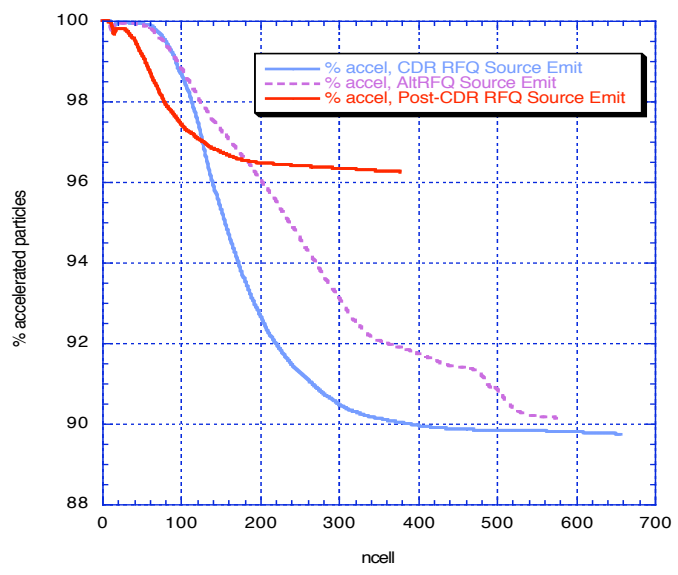

Fig. 6.7-2. \% accelerated particles vs. cell number, Source Emittance initial distribution.

As shown in Section 3.1, at the design conditions, the three RFQs are rather similar; for the ion source emittance distribution of $\sim 1 \mathrm{M}$ particles rms matched to the RFQ input, the percent of particles lost with energy $\geq 1 \mathrm{MeV}$ is:
Post-CDR
$0.081 \%$ loss above $1 \mathrm{MeV}$
CDR
$0.073 \%$ loss above $1 \mathrm{MeV}$
AltCdr
$0.123 \%$ loss above $1 \mathrm{MeV}$

The longer CDR RFQ accumulates about the same amount of losses with energies $>$ $1 \mathrm{MeV}$ as the shorter Post-CDR RFQ. The AltCDR RFQ has larger losses between $1 \mathrm{MeV}$ and $3 \mathrm{MeV}$, and less losses with energies above $3 \mathrm{MeV}$. 


\subsection{Sensitivity Comparisons}

Sensitivities of the percentage of accelerated beam and the percentage of losses above 1 $\mathrm{MeV}$ were explored with $100 \mathrm{~K}$ particle runs for variations of input emittance, input current, and the input emittance alpha and beta parameters. As noted in Section 8, $10 \mathrm{~K}$ particles is not enough for reliable loss-above-1-MeV statistics, but the running time for $100 \mathrm{~K}$ particles is long.

The same vertical scales are used for each group of plots to aid the eye in comparing the RFQs. Red - \% accelerated beam; blue, \% >1MeV losses.

\subsubsection{Variation of Input Emittance and Input Current}
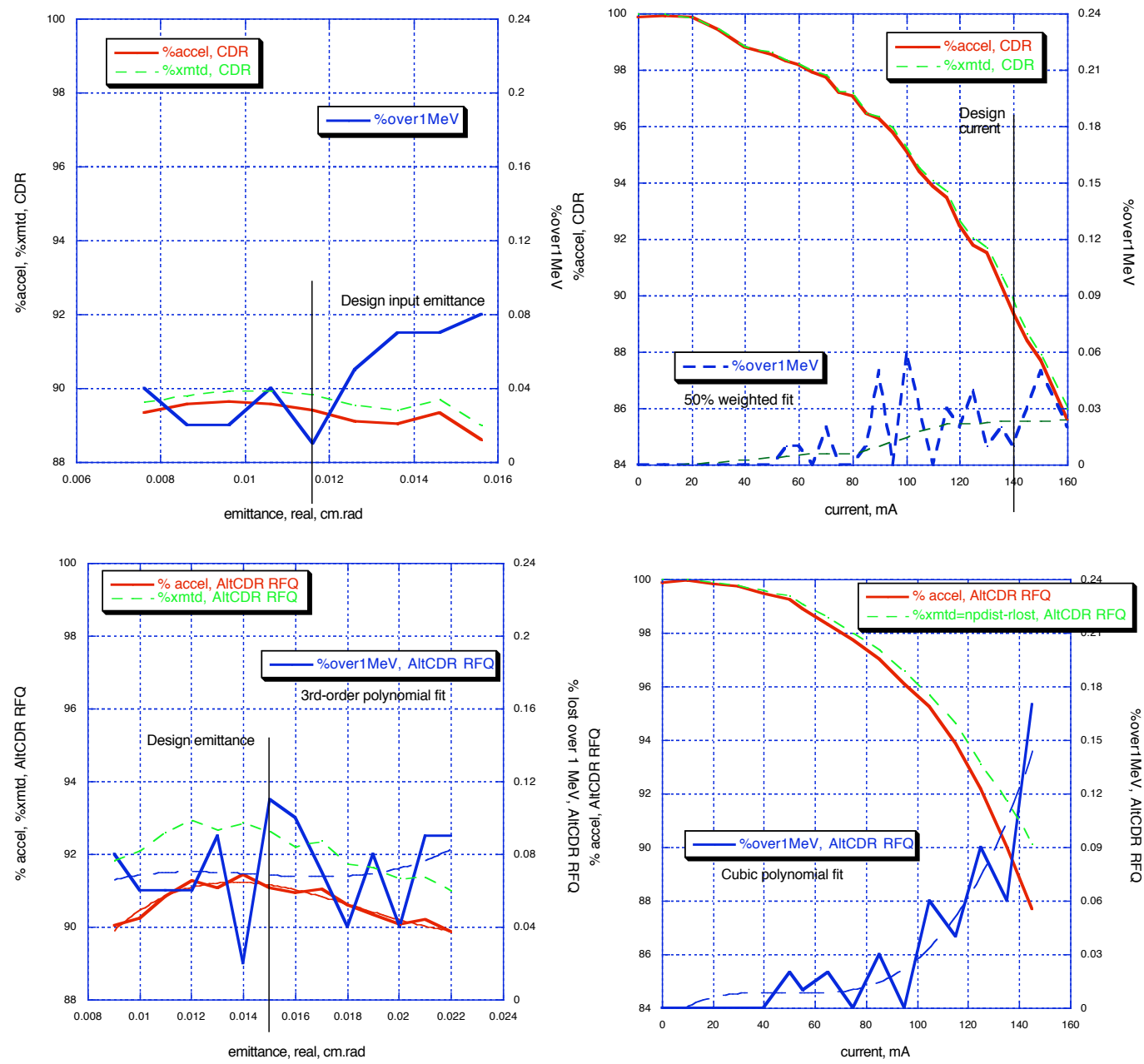


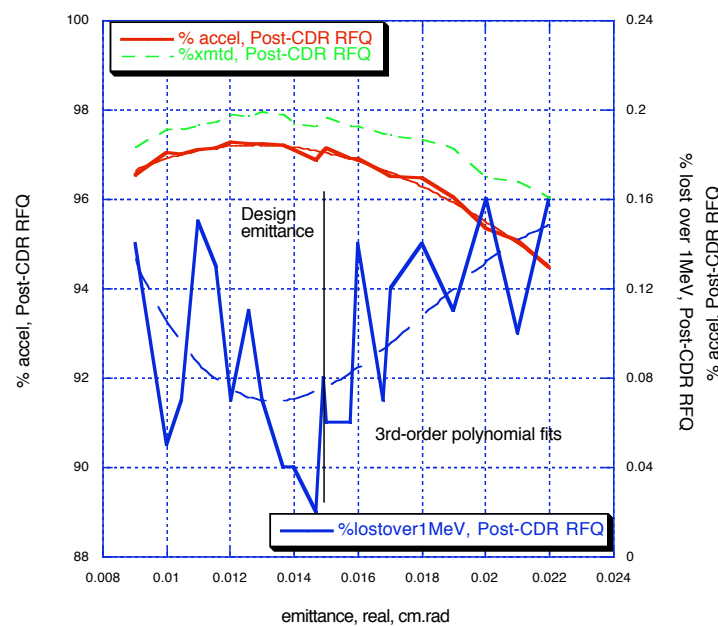

Figs. 6.8-1-a,b,c. Variation with input emittance area.

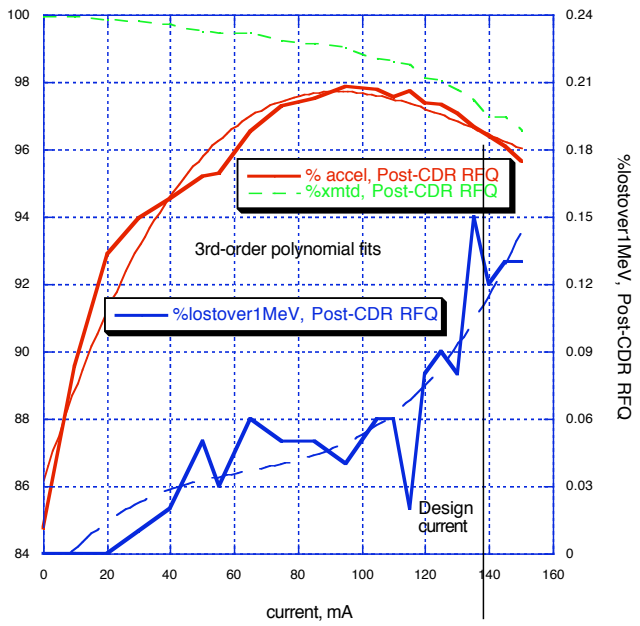

Figs. 6.8-2-a,b,c. Variation with input current.

The $>1 \mathrm{MeV}$-losses of the longer CDR RFQ and especially the AltCDR RFQ are quite insensitive to the input emittance area.

The long CDR RFQ has relatively low $>1 \mathrm{MeV}$-losses sensitivity to the input current. The AltCDR and Post-CDR RFQs are similar to each other.

The Post-CDR RFQ exhibits a decline in the percentage of accelerated beam as the input current is reduced. This is unusual. The behavior is the same for very small input emittance and current - the decline in accelerated beam fraction is a consequence of the quite short shaper, which, however, provided a high \%-accelerated beam and acceptable $>1 \mathrm{MeV}-$ losses at the design current. 


\subsubsection{Variation of Accelerated Beam with Input Emittance $\alpha$ and $\beta$}

These figures use the $100 \mathrm{~K}$ input distribution - see Section 8.2.4.
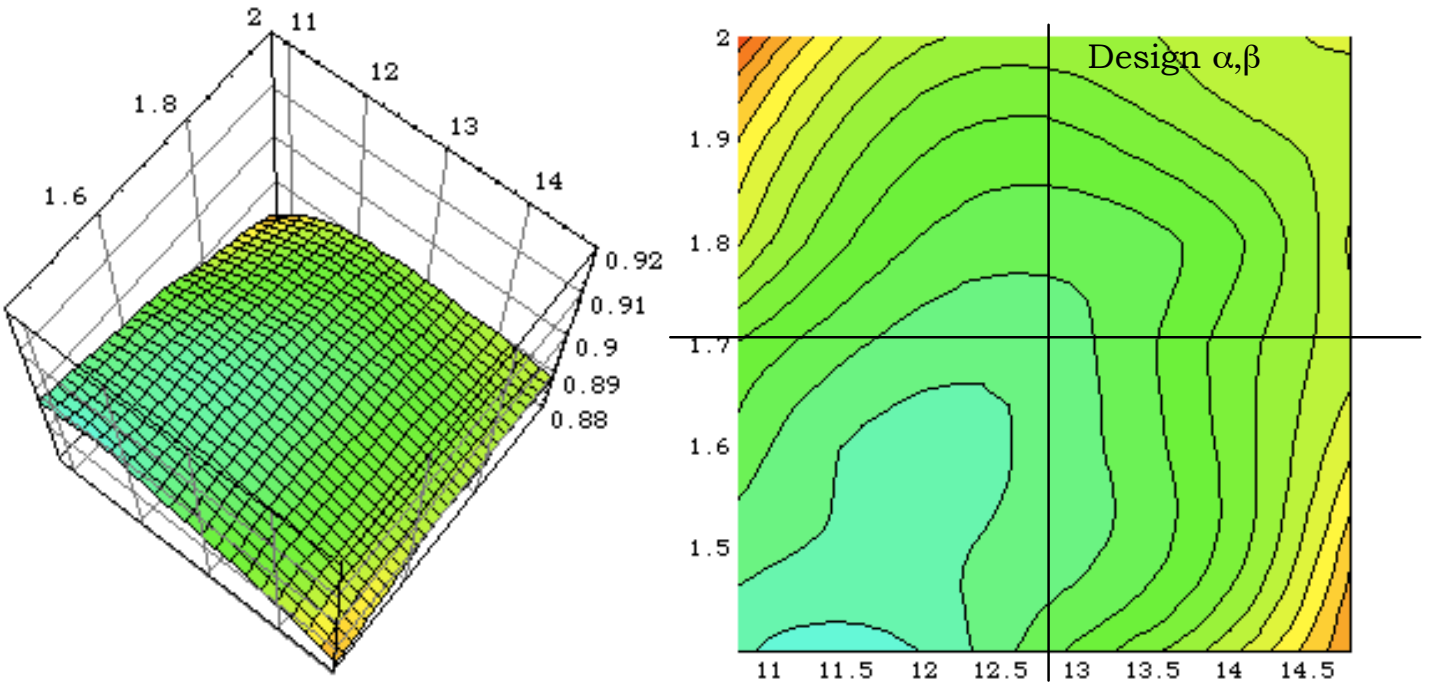

Fig.6.8-3. CDR RFQ - \% Accelerated beam sensitivity to input emittance $\alpha$ and $\beta$. The design $\alpha$ and $\beta$ are as found for the original CDR RFQ evaluated without multipole or image fields. At the design $\alpha=1.7008, \beta=12.7828$, $\{\%$ AccBeam, \%Loss $>1 \mathrm{MeV}\}$ is $\{89.6,0.034\}$. At $\alpha=1.4, \beta=$ 11.5, \{89.9,0.030\}; at $\alpha=1.4, \beta=12.75,\{89.6,0.014\}$; (Fig. 6.8-6).
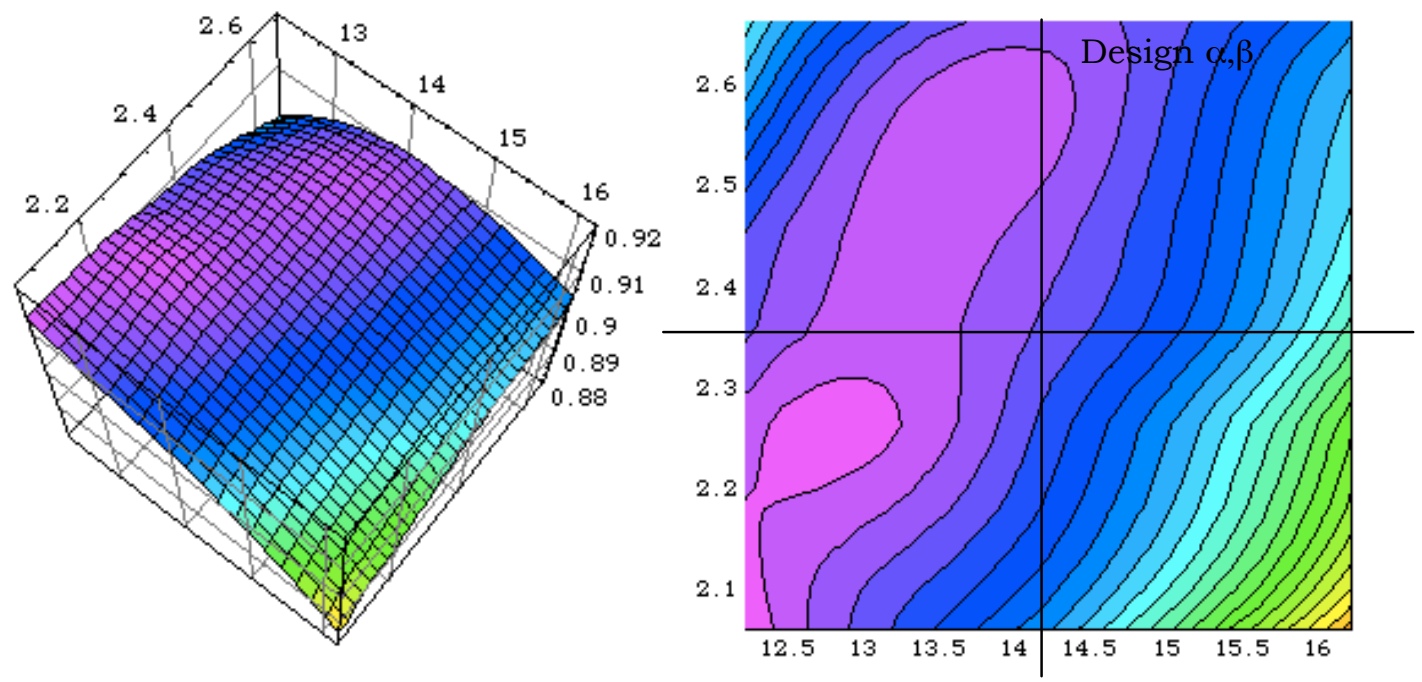

Fig.6.8-4. AltCDR RFQ - Accelerated beam sensitivity to input emittance $\alpha$ and $\beta$. At the design $\alpha=2.362, \beta=14.2$, \{\%AccBeam, \%Loss $>1 \mathrm{MeV}\}$ is $\{90.0,0.066\}$. At $\alpha=2.25, \beta=$ 12.75, $\{91.3,0.080\}$; at $\alpha=2.47, \beta=15$., \{90.7,0.058\}; (Fig. 6.8-7). 

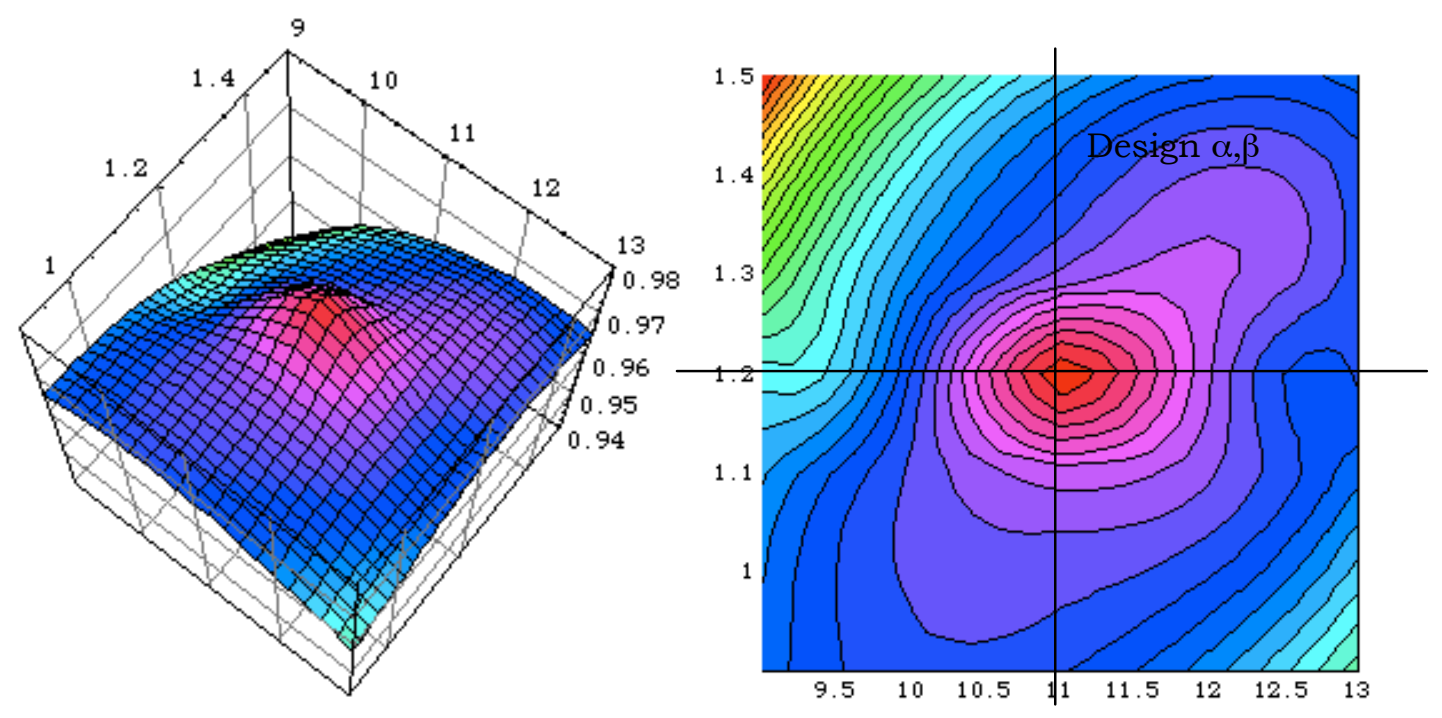

Fig.6.8-5. Post-CDR RFQ - Accelerated beam sensitivity to input emittance $\alpha$ and $\beta$. The design $\alpha=1.2, \beta=11$, as found during the optimization; $\{\%$ AccBeam, \%Loss $>1 \mathrm{MeV}\}$ is $\{98.0,0.101\}$. At $\alpha=1.3, \beta=10.9,\{97.0,0.074\}$; at $\alpha=1.1, \beta=10.1,\{96.9,0.076\}$; Fig. $6.8-8)$.

Variation of $>1 \mathrm{MeV}$ losses with input emittance $\alpha$ and $\beta$ is shown in the next section. It is seen that the matching for maximum accelerated beam percentage is generally not the same as a match for minimum $>1 \mathrm{MeV}$ losses.

The CDR and AltCDR RFQs have similar sensitivity of the accelerated beam percentage to the match. The Post CDR RFQ is more sensitive, as expected from pushing the design toward shorter length. 


\subsubsection{Variation of $>1 \mathrm{MeV}$ Losses with Input Emittance $\alpha$ and $\beta$}
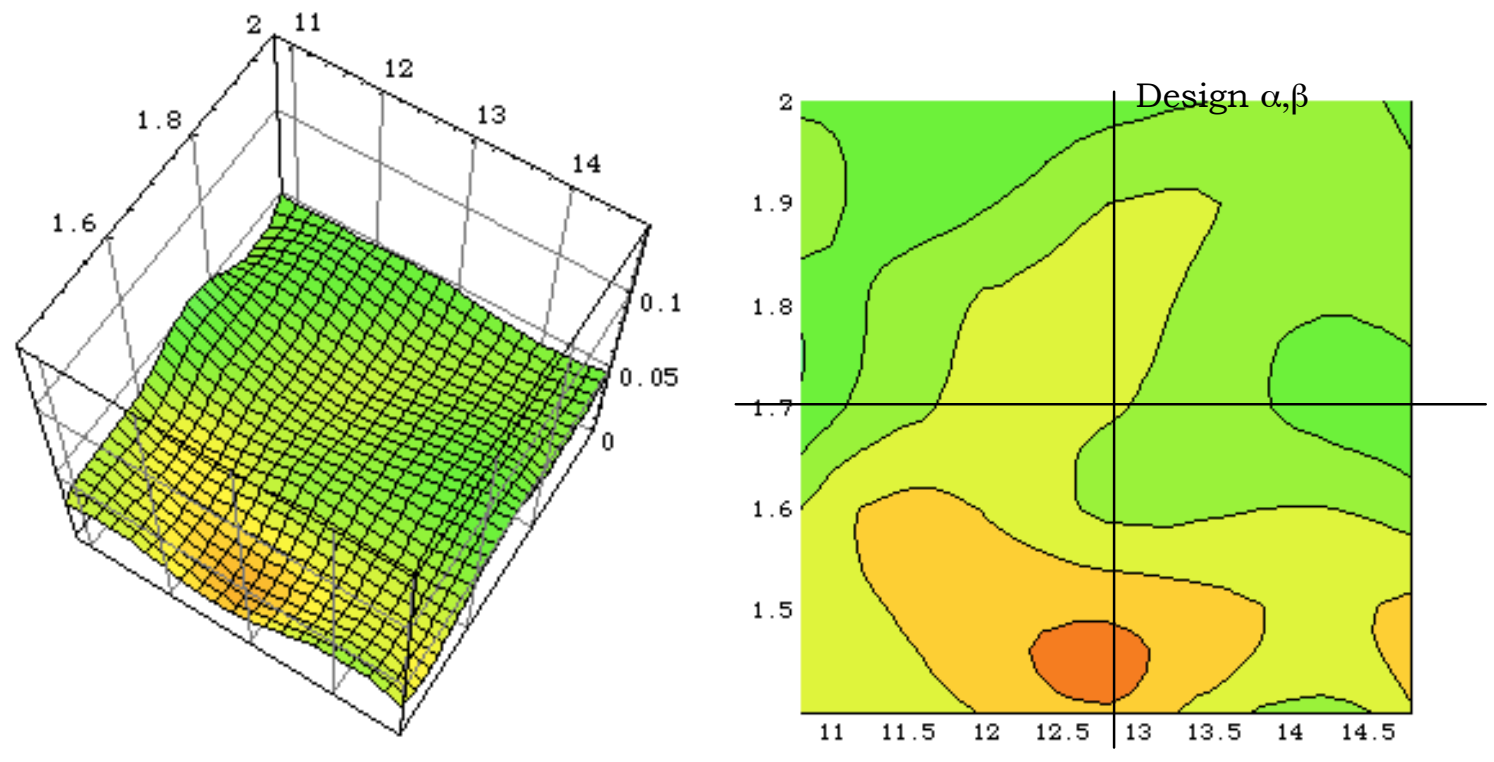

Fig.6.8-6. CDR RFQ - >1MeV-losses sensitivity to input emittance $\alpha$ and $\beta$. At the design $\alpha=1.7008, \beta=12.7828$, $\{\%$ AccBeam, \%Loss $>1 \mathrm{MeV}\}$ is $\{89.6,0.034\}$. At $\alpha=1.4, \mathrm{~b}=11.5$, $\{89.9,0.030\}$; at $\alpha=1.4, \beta=12.75$, \{89.6,0.014\}; (Fig. 6.8-3).
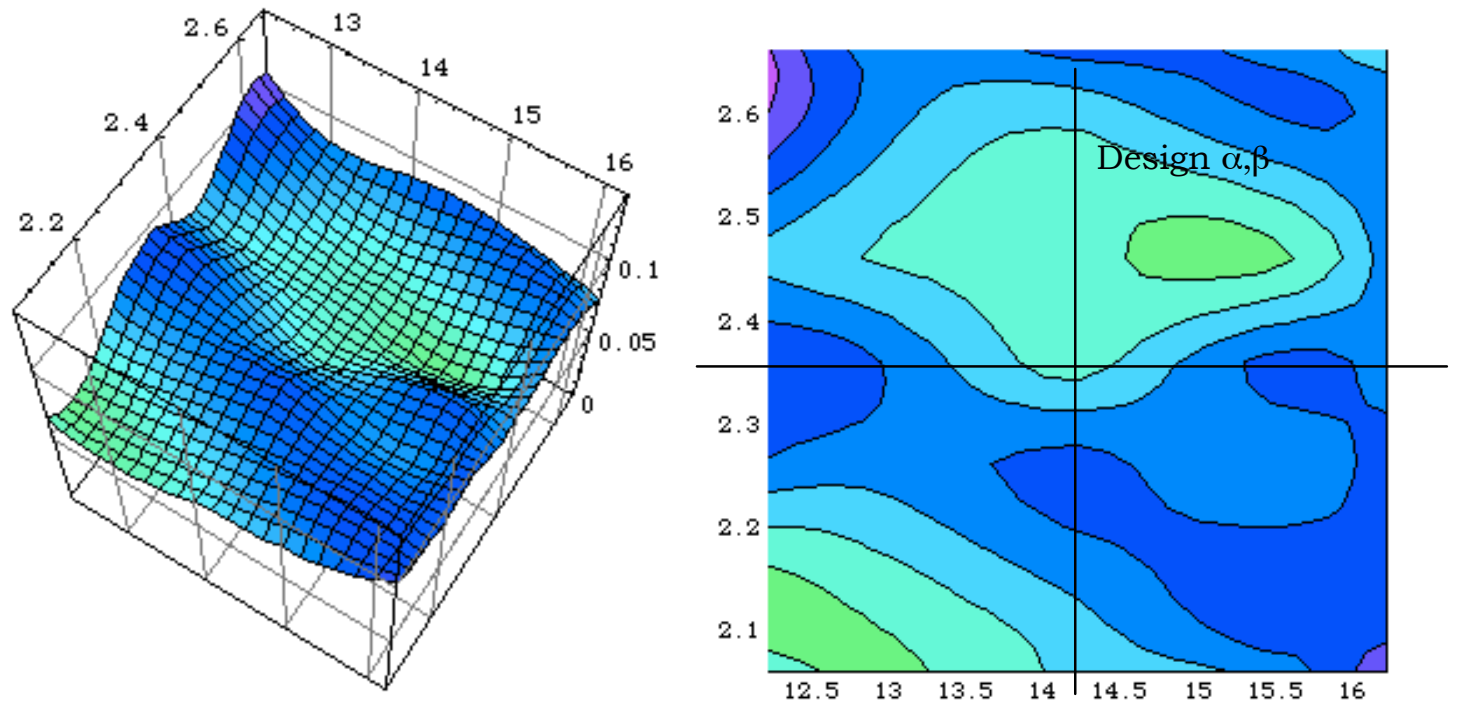

Fig.6.8-7. AltCDR RFQ - >1MeV-losses sensitivity to input emittance $\alpha$ and $\beta$. At the design $\alpha=2.362, \beta=14.2$, $\beta$ AccBeam, \%Loss $>1 \mathrm{MeV}\}$ is $\{90.0,0.066\}$. At $\alpha=2.25, \beta=$ $12.75,\{91.3,0.080\}$; at $\alpha=2.47, \beta=15 .,\{90.7,0.058\}$; (Fig. 6.8-4).

The trend of the $>1 \mathrm{MeV}$ losses to the match is similar for all three RFQs. The exact values are approximate even with $100 \mathrm{~K}$ particles per point - see the discussion on the number of particles simulated in Section 8.2.4 

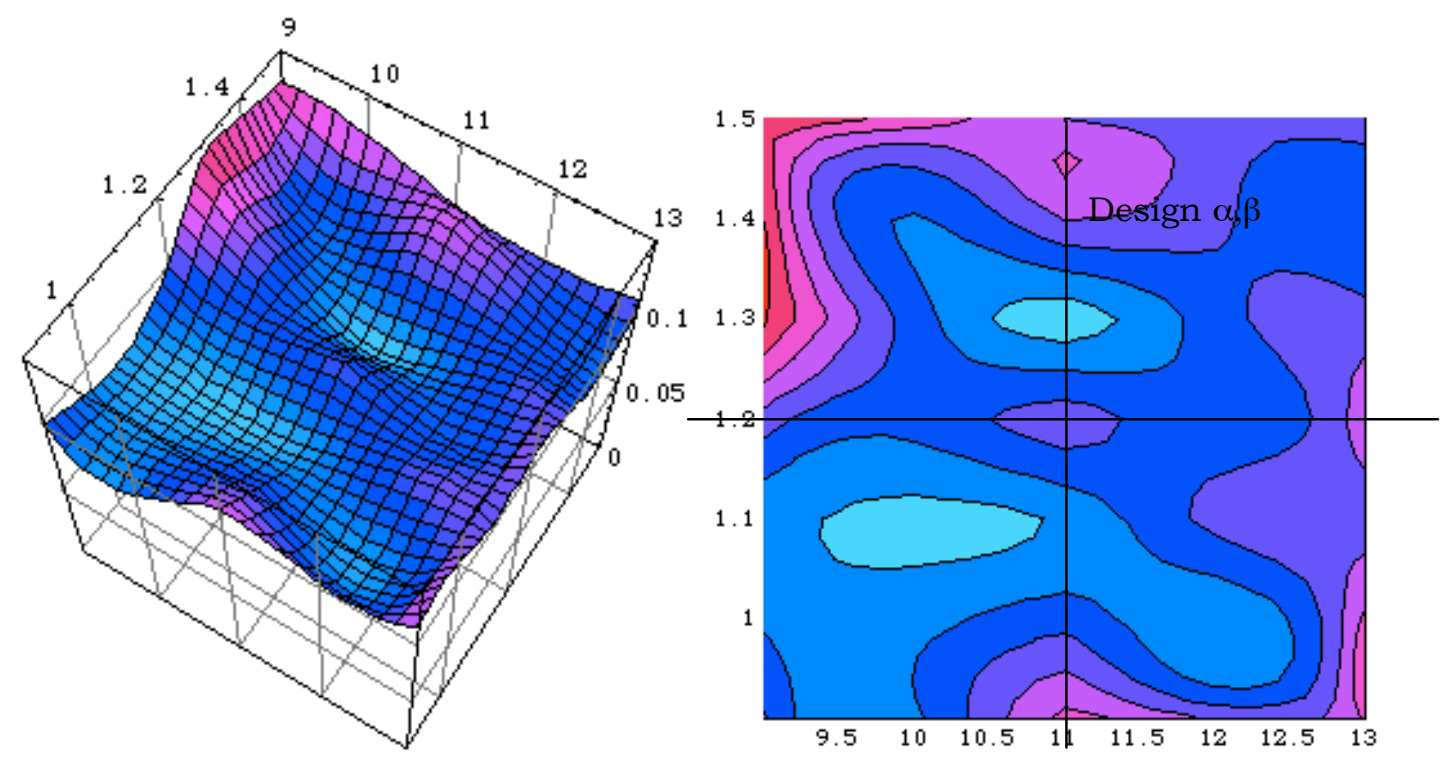

Fig.6.8-8. Post-CDR RFQ - >1MeV-losses sensitivity to input emittance $\alpha$ and $\beta$. At the design $\alpha=1.2, \beta=11$, $\{$ AccBeam, \%Loss $>1 \mathrm{MeV}\}$ is $\{98.0,0.101\}$. At $\alpha=1.3, \beta=10.9$, $\{97.0,0.074\}$; at $\alpha=1.1, \beta=10.1,\{96.9,0.076\} ;$ (Fig. 6.8-5).

\subsection{Comparison Summary}

Table 6.9-1 Summary of design conditions, strategy and optimization (a.-e.) and prioritized results (1.-5.) at the design match

\begin{tabular}{|l|c|c|c|}
\hline & CDR RFQ & AltCDR RFQ & Post-CDR RFQ \\
\hline a. Input Beam Current, mA & 140 & 130 & 130 \\
\hline $\begin{array}{l}\text { b. Input Beam norm rms } \\
\text { emittance, mm.mrad }\end{array}$ & 0.2 & 0.25 & 0.25 \\
\hline c. Input energy, MeV & 0.100 & 0.095 & 0.095 \\
\hline d. Design Strategy & EP & Conventional & EP \\
\hline e. Optimized & $\begin{array}{c}\text { Yes (without } \\
\text { multipoles, } \\
\text { images) }\end{array}$ & No & $\begin{array}{c}\text { Yes (with } \\
\text { multipoles, } \\
\text { images) }\end{array}$ \\
\hline $\begin{array}{l}\text { 1. \% loss > 1 MeV, Ion Source } \\
\text { Input Distribution }\end{array}$ & 0.073 & 0.123 & 0.081 \\
\hline 2. RF Power, MW & $\sim 1.1$ & $\sim 1.0$ & $\sim 1.1$ \\
\hline 3. Peak Field KP Factor & 1.7 & 1.8 & 1.7 \\
\hline 4. Length, m & $\sim 12.3$ & $\sim 12.1$ & $\sim 8$ \\
\hline $\begin{array}{l}\text { 5. \% Accelerated Beam, Ion } \\
\text { Source Input Distribution }\end{array}$ & 89.4 & 89.9 & 95.8 \\
\hline
\end{tabular}

\subsubsection{Design Conditions, Strategy and Optimization}

a. Input Beam Current - The $140 \mathrm{~mA}$ design current for the CDA/CDR RFQ was lowered to $130 \mathrm{~mA}$ for the AltCDR RFQ, and also adopted for the Post=CDR RFQ. This 
reflected confidence in obtaining $125 \mathrm{~mA}$ accelerated current, requiring $~ 96.2 \%$ accelerated beam fraction.

The Post-CDR meets this goal, depending on how much the ion-source/LEBT injected beam resembles the ideal waterbag distribution.

Re-optimization of the CDR RFQ would probably also reach this performance, as might also an optimization of the AltCDR RFQ

b. Input beam normalized rms emittance - The $0.2 \mathrm{~mm}$.mrad CDA/CDR RFQdesign input emittance was raised to 0.25 for the AltCDR RFQ, and also adopted for the PostCDR RFQ. This reflected a revised estimate of the emittance expected from the ionsource/LEBT. The larger value should ease space-charge somewhat.

c. Input beam energy - The $100 \mathrm{keV}$ CDA/CDR RFQ input energy was lowered to 95 $\mathrm{keV}$ for the AltCDR RFQ based on the ECR ion source performance, and also adopted for the Post-CDR RFQ. This raises the space-charge at injection, and in general would result in a shorter RFQ.

Design conditions a.-c. result in the CDR RFQ having the hardest specification. However, they appear not to result in much difference between the three designs.

\section{d. Design strategy -}

CDR RFQ - The CDR RFQ maintains the beam in equilibrium (equipartitioned) from the end of the shaper to the end of the RFQ. There are no interactions with major resonances.

AltCDR RFQ - The conventional design strategy results in a long RFQ and in longitudinal interaction with major resonances.

Post-CDR RFQ - A shorter RFQ that could meet the other specifications with approximately the same rf power requirement as the CDR design was sought. The PostCDR RFQ maintains the beam in equilibrium (equipartitioned) from the end of the shaper to the end of the RFQ. There are no interactions with major resonances.

\section{e. Optimization -}

CDR RFQ - The design was optimized using the 2-term potential description, without multipole and image forces. Introduction of these forces lowered the accelerated beam fraction, and therefore a new design was sought.

The simplest re-optimization would have been to keep the rules which result in $\sim 12 \mathrm{~m}$ length, and only re-optimize the aperture at the end of the shaper, perhaps accompanied by some adjustment of the shaper length and porch. It is known that slight changes in the aperture and modulation to restore the quadrupole and acceleration potential terms to their 2-term value will result in essentially the 2-term performance - this procedure is implemented in PARMTEQM. Probably this re- 
optimization would then result in increased accelerated beam fraction with little change in the other characteristics.

Instead, a new, shorter design was sought, resulting in the Post-CDR design.

AltCDR RFQ - Not optimized. There is little experience with optimization to these or other criteria using the conventional design approach, so it is not clear what could be gained by further work in this direction.

Post-CDR RFQ - The design was optimized using the analytical potential description including multipole and image forces, seeking shorter length with no compromise of the other specifications.

\subsubsection{Prioritized Design Results}
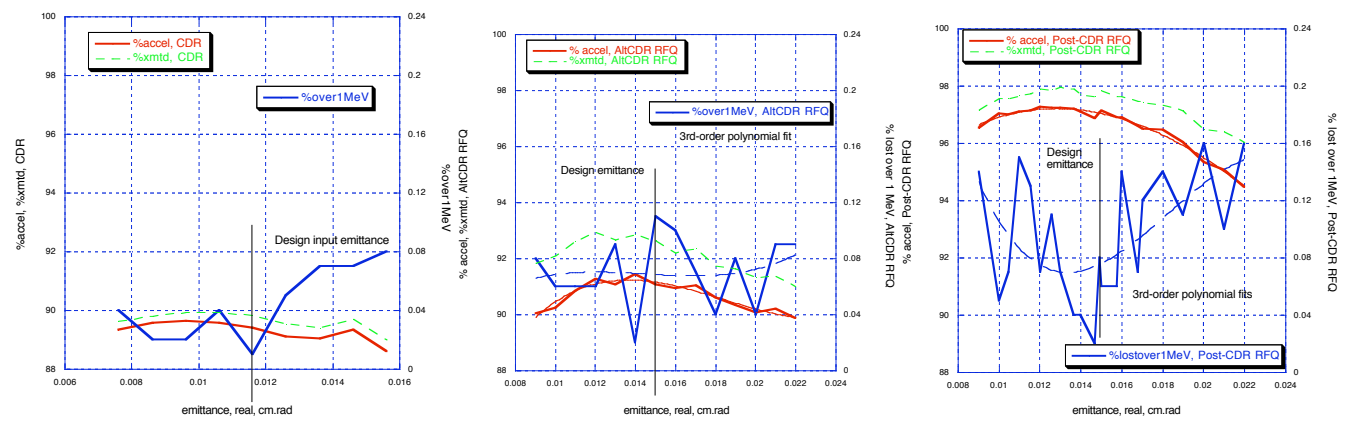

Fig. 6.9-1. Sensitivity of accelerated beam fraction and $\%>1 \mathrm{MeV}-10$ sses to input emittance. Repeat of fig. 6.8-1; left-to-right CDR, AltCDR, Post-CDR.
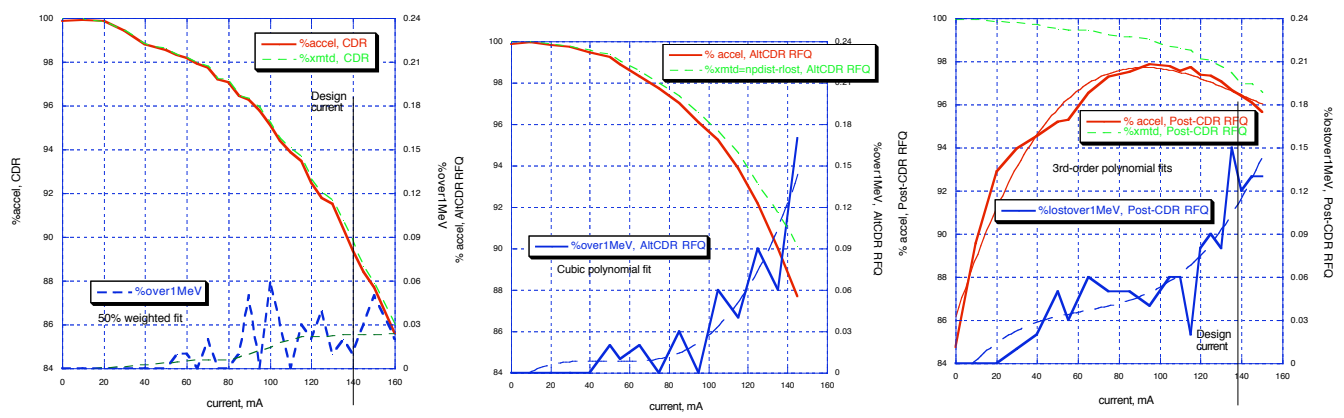

Fig. 6.9-2. Sensitivity of accelerated beam fraction and $\%>1 \mathrm{MeV}-$ losses to input current. Repeat of fig. 6.8-2; left-to-right CDR, AltCDR, Post-CDR. 

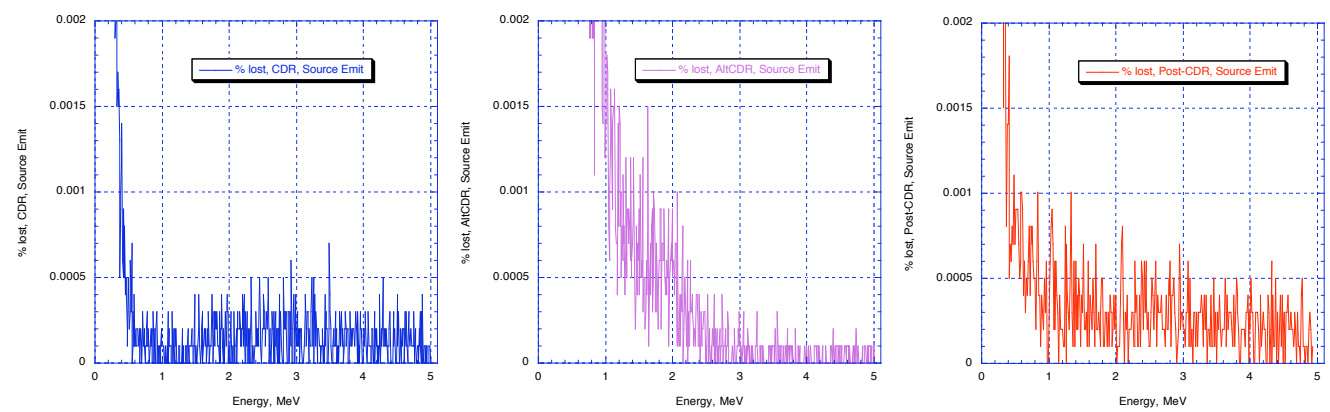

Fig. 6.9-3. Loss patterns for $\sim 1 \mathrm{M}$ particle Source emittance input distribution, repeat of Fig. 3.1-3. It is necessary to use a simulation code with canonical (time) coordinates for accurate loss patterns. Left-to-right CDR, AltCDR, Post-CDR.
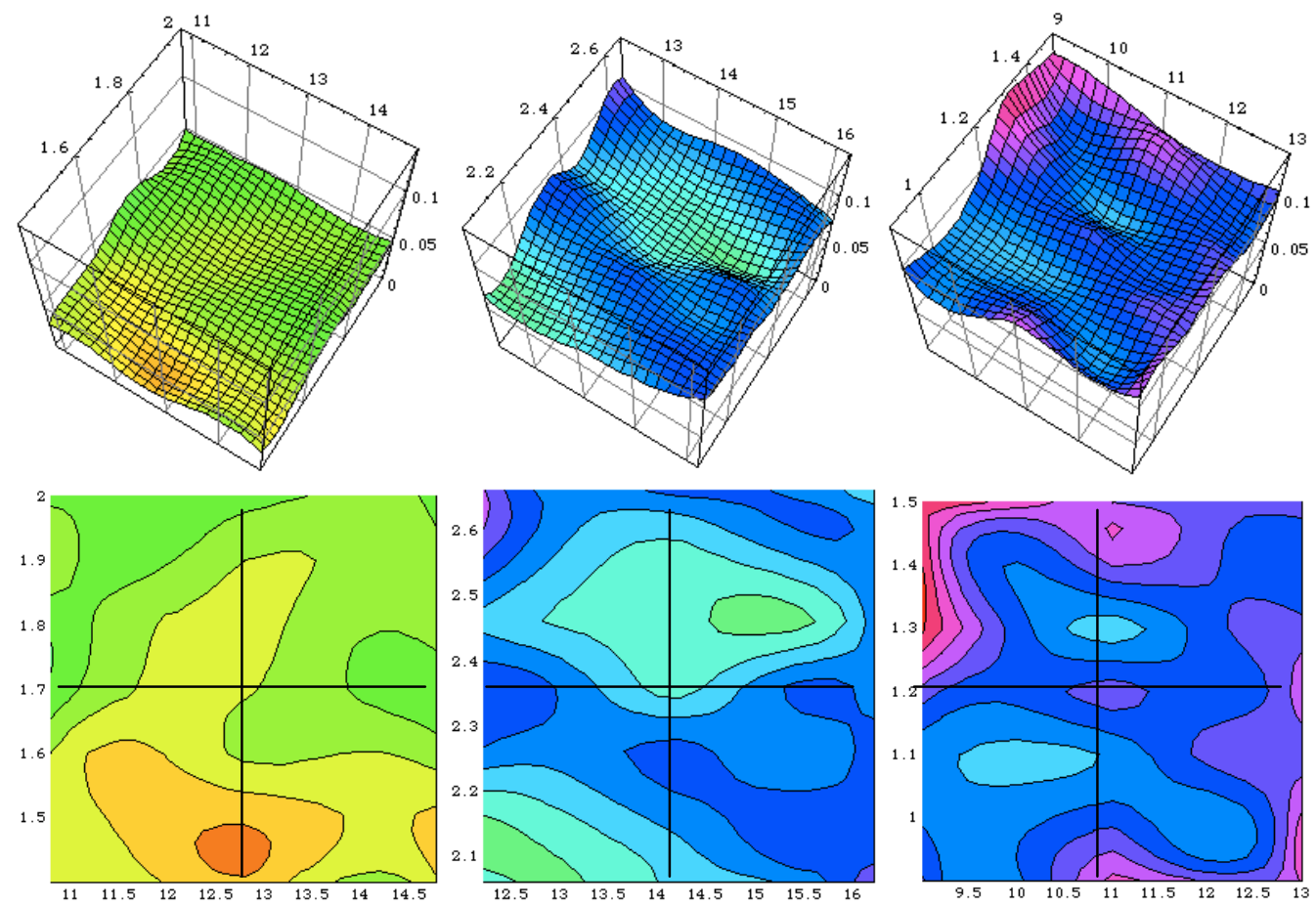

Fig. 6.9-4. \% losses $>1 \mathrm{MeV}$, repeat of figs. 6.8-6,7,8. Left-to-right CDR, AltCDR, Post-CDR. Produced from $100 \mathrm{~K}$ particle simulations.

The long, more slowly varying vane profile and resonance-free dynamics of the CDR RFQ appear to result in low and evenly distributed losses with energies $>1 \mathrm{MeV}$. The sensitivities with respect to input emittance, current and matching are also the smallest of the three RFQs.

The conventional AltCDR RFQ has somewhat higher total losses $>1 \mathrm{MeV}$, concentrated between $1-3 \mathrm{MeV}$ and less from $3-5 \mathrm{MeV}$, compared to the other designs. This may be the result of the aperture choke-point discussed in Section 4.3 and 4.4. The lower 3$5 \mathrm{MeV}$ losses result from the strong transverse focusing in the accelerator section. 
The Post-CDR RFQ design strategy sought shorter length, and uses a varying EP recipe and shorter shaper. The $>1 \mathrm{MeV}$ losses at the design point are similar to the other two designs. The sensitivity is greater that for the CDR RFQ, and similar to the AltCDR RFQ but for different reasons.

\section{RF Power, $M W$}

The rf power of the three design are similar. Note the discussion about the rf power estimate in Section 7.

\section{Peak Field KP Factor}

Contrary to what was thought in the RFQ early days, it is not necessary to use the highest fields feasible without sparking, i.e., the highest KP factor. It is only necessary to provide enough voltage to achieve an optimum performance. It is easier to match the LEBT beam to the RFQ input with weaker initial focusing, so the end-of-shaper (EOS) voltage is a good voltage for the whole shaper. The CDR and AltCDR RFQs rising voltage from the EOS back to the beginning of the RFQ is not necessary, and results in wasted $\mathrm{rf}$ power and harder input matching (as evidenced from the design input ellipse matching parameters). The $1.8 \mathrm{KP}$ factor used in the front part of the AltCDR RFQ also appears unnecessary. An optimization following the lines of the Post-CDR with $1.8 \mathrm{KP}$ factor was pursued - similar beam performance and length were obtained, but considerably more $\mathrm{rf}$ power was required

As noted, further design optimization work involving the Rho/r0 ratio could be fruitful.

\section{Length}

The Post-CDR design has two-thirds the length of the other two, with better percentage of accelerated beam and about the same percent of losses above $1 \mathrm{MeV}$. There would be a saving in the initial construction cost of the RFQ. The length is relatively unimportant in terms of construction and tuning technique.

\section{5. \% Accelerated Beam, Ion Source Input Distribution}

The percentage of accelerated beam is highest for the Post-CDR design, and reoptimization of the CDR approach could probably reach this level. As the ion source should produce at least $140 \mathrm{~mA}$, the percent of accelerated beam is not as important as the percentage of lost beam $>1 \mathrm{MeV}$. The CDR and AltCDR RFQs have similar sensitivity of the accelerated beam percentage to the match. The Post CDR RFQ is more sensitive, as expected from pushing the design toward shorter length. 

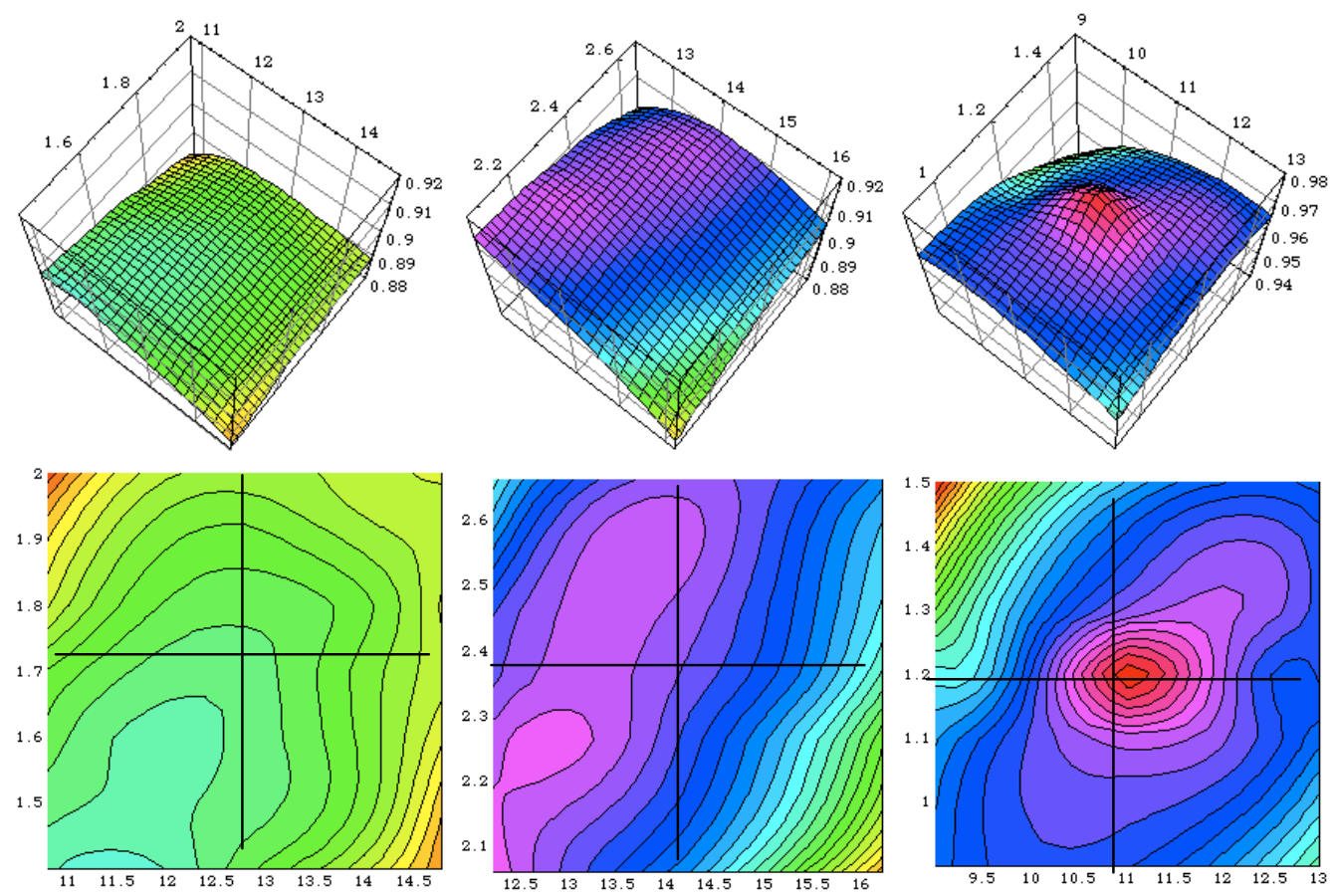

Fig. 6.9-5. Accelerated particles from 100K waterbag input distribution, repeat of Figs. 6.86,7,8. Left-to-right CDR, AltCDR, Post-CDR. (Different scale for PostCDR)

\subsubsection{Overall Summary and Comment}

The Post-CDR design satisfies the beam loss and transmission specifications with shorter length and no sacrifice in rf power. It appears to be somewhat more sensitive to input parameter variations than the CDR design, but this should not be an operational problem in a well-controlled environment. It represents a lower bound on the length while keeping the rf power low.

Overall, the AltRFQ performance is good, but it is long, and more important, the longitudinal dynamics involving interaction with strong resonances is troublesome. The strongly growing longitudinal beam size and emittance do not cause problems in the RFQ, but may in the following $5-40 \mathrm{MeV}$ linac or in the HEBT. They also may be sensitive to other errors such as vane manufacturing or alignment errors, not studied here. As the resonance interaction is generic to the conventional approach, it is not clear that better characteristics could be achieved. It is also not apparent that other advantages would be gained by some approach to optimization. 

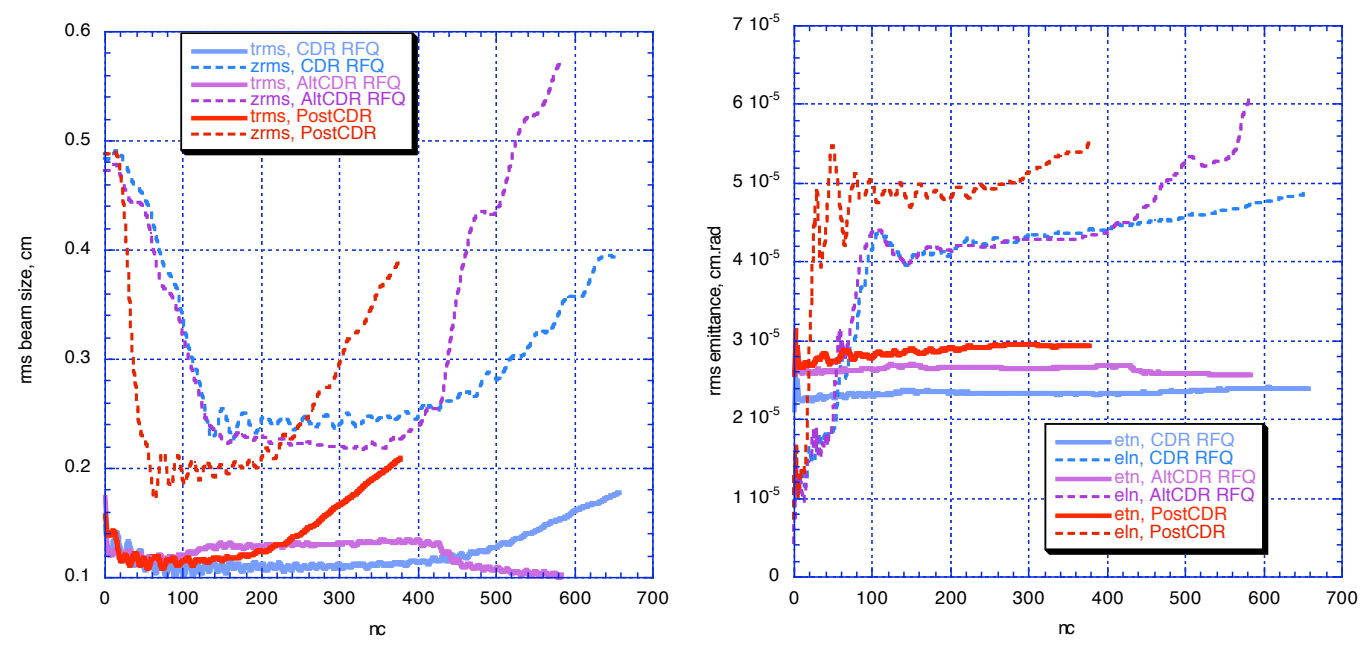

Fig. 6.9.3-1. Repeat of Figs. 6.4-1 and 6.4-2. Rms beam size and emittance comparisons.

The CDR design appears to have lower losses $>1 \mathrm{MeV}$ and less sensitivity; higher transmission can be regained by re-optimization. Using the EOS vane voltage for the whole shaper would result in some saving of $\mathrm{rf}$ power and make input matching even less sensitive.

Suggestions for further work toward a final design:

1. Pursue the LEBT design to conclusion, and obtain a truly representative input beam distribution.

The EP design strategy is intrinsically robust.

2. Re-optimize the CDR RFQ as suggested in Section 6.8. This is a relatively minor job, and would give a bound on the performance of an IFMIF RFQ $~ 12$ meters long. Some saving in $\mathrm{rf}$ power would result.

3. Investigate designs intermediate to the CDR and Post-CDR designs. A somewhat larger increase in aperture might reduce the $>1 \mathrm{MeV}$ loss. The best aperture increase, synchronous phase and EP/emittance-control rules might be other than the simple functions used to date - evidence points toward further investigation of very subtle factors such as adiabaticity. The overall transverse focusing that gives optimum $>1$ $\mathrm{MeV}$ loss and acceleration percentage also has subtleties that require further research, which might result in $\mathrm{rf}$ power savings. Rf power cost saving could easily override length cost saving. Include investigation of the $\mathrm{Rho} / \mathrm{rO}$ factor. 


\section{RFQ Vane Voltage, RF Power}

\subsection{Variable Vane Voltage Profile}

Freedom to vary the vane voltage is a powerful tool for the designer. Modern construction and tuning techniques afford this option. For example, the LEDA RFQ used tapered vane skirts to vary the voltage. The IFMIF CDR and Post-CDR designs were checked by experts who have successfully built and operated RFQs with a varying vane voltage profile [45]. Their view is that the varying voltage profile can be achieved by the vane design and tuning techniques, and that the power loss per unit length, while possibly challenging, can also be cooled satisfactorily [46].

\subsubsection{The Russian IFMIF CDA Preliminary RFQ Proposal}

In this regard and as a conceptually different and interesting design approach, the preliminary proposal sketched by the Russian IFMIF partner in the IFMIF CDA, Section 2.6, is important [47]. The parameter table is shown in Fig. 7.1.1-1:

Table 2.6.8-1. Parameters of the RFQ-SPRFQ design for IFMIF

\begin{tabular}{lcc}
\hline \multicolumn{1}{c}{ Parameters } & RFQ & SPRFQ \\
\hline Frequency, $\mathrm{MHz}$ & 175 & 175 \\
Input energy, MeV & 0.1 & 3.0 \\
Output energy, MeV & 3.0 & 8.0 \\
Intervane voltage, $\mathrm{kV}$ & 87. & 210. \\
Characteristic bore radius, mm & 4.2 & 4.2 \\
Aperture radius, mm & $4.19-3.20$ & 4.0 \\
Length, m & 4.97 & 2.4 \\
Input beam current, mA & 130 & 126.2 \\
Transmission efficiency, \% & 97.8 & 100 \\
Input normalized beam emittance, $\pi \mathrm{mm}$ mrad & 0.6 & 0.9 \\
Output normalized beam emittance, $\pi$ mm mrad & 0.8 & 0.9 \\
Surface field, $\mathrm{E}_{\max }, \mathrm{kVcm}^{-1}$ & 280 & 310 \\
\hline
\end{tabular}

Fig. 7.1.1-1. Parameter table for 0.1-3.0 MeV and 3.0-8.0 MeV RFQs for IFMIF proposed by IHEP.

It is stated that "Capacitance is added in a controlled manner through the SPRFQ, allowing a large increase in the vane voltage, up to factors of 3-5 or more, limited by rf power loss considerations. The technique allows the SPRFQ to be considerably shorter, and total $\mathrm{rf}$ power losses to be less.

The combined parameters, including the length, are similar to those of the Post-CDR RFQ design. The IHEP approach, outlined further in [48], pp. 65-77 and including the two-tank approach, is worthy of further investigation by the IFMIF project unfortunately lack of funding prevented this to date. 


\subsection{RF Copper Power Consumption}

The estimates of the rf power required by the RFQ structure given in Fig. 2.4-9,10 are comparatively correct, but the actual values present some questions, and require further attention by the IFMIF design team. These estimates are based on [49], which is essentially repeated here.

\subsubsection{RF Shunt Impedance Estimate}

\section{RFQUIK Estimate}

The RFQ design code RFQUIK has a formula for shunt impedance:

( RS RFQUIK, $M \Omega \mathrm{m}$ ) =

$1 /\left(\left(\left(1.3 * 10^{\wedge} 12 * 1.25 *(\right.\right.\right.$ ( frequency, $\mathrm{MHz}) * 48 * 10^{\wedge} \quad-$

$12 *((300 /($ freq, $\mathrm{MHz})) /$

( (cell aperture, $\mathrm{m})$ *

$(1+$ modulation $\left.\left.\left.\left.) /(2)))^{\wedge}(1 / 6)\right)^{\wedge}(3 / 2)\right)\right) / 0.6\right)$

where $\mathrm{r} 0 \approx$ aperture $(1+$ modulation $) / 2 ; 0.6$ is a "fudge factor" to account for observed differences between theoretical and measured results.

Fig. 7.2.1-1 shows the (Rs RFQUIK) estimate along the IFMIF CDR RFQ

IFMIF EP KP1.7 RFQ tapeoutstd

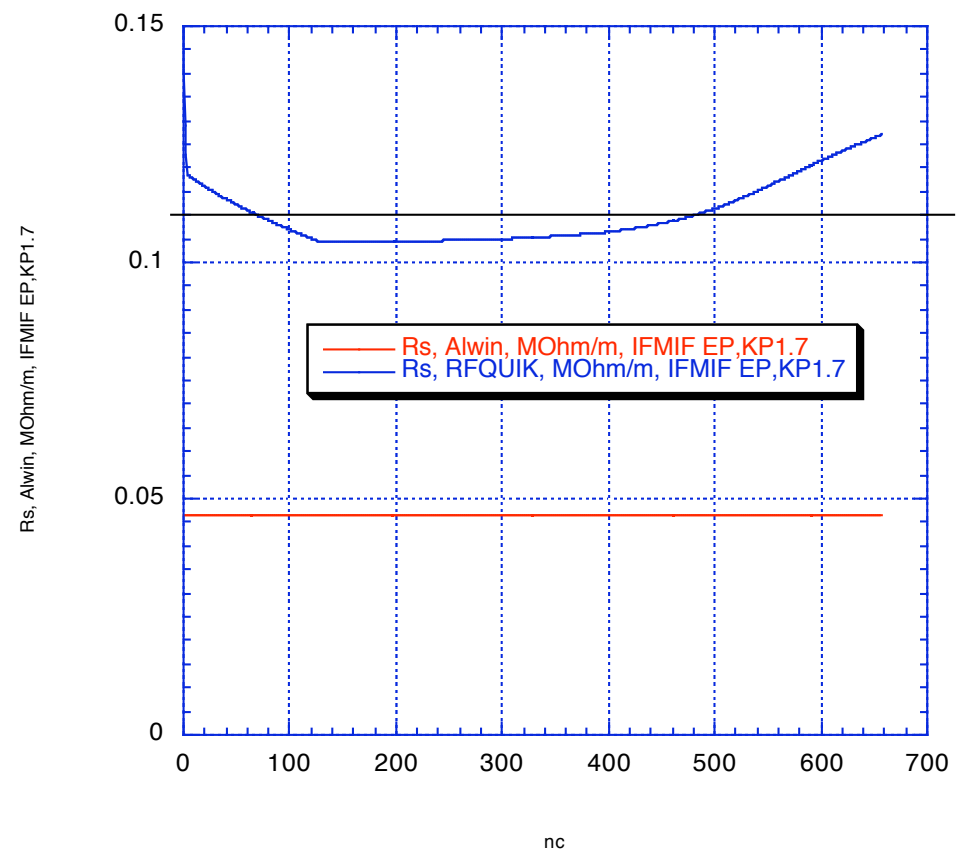

Fig. 7.2.1-1. RFQUIK estimate for rf shunt impedance Rs along the IFMIF CDR RFQ.

$($ CuPwr/cell RFQUIK $)=$

(cell length,m)*(vane voltage, MV)^2/(Rs RFQUIK)

These equations are integrated along the RFQ to get the total copper power estimate: 
Total Copper Power RFQUIK = 1.125 MW

Beam Power

Assume Beam Power $=(4.905 \mathrm{MeV})(.132 \mathrm{~A})(.95$ transmission $)=(4.905)(.125) \approx 620 \mathrm{~kW}$

IAP Estimate

IAP presentation at the EU Monitoring $2 / 2003$ gives total RFQ power $=1.5 \mathrm{MW}$; minus $620 \mathrm{~kW}=880 \mathrm{~kW} R F Q$ copper power.

Saclay Estimate

From Saclay presentations on KEP results,

Main IFMIF RFQ parameters

\begin{tabular}{|c|c|c|c|}
\hline Parameters & Values & Parameters & Values \\
\hline Length & $12.482 \mathrm{~m}$ & Synchronous phase & $-90^{\circ} \rightarrow-40^{\circ}$ \\
\hline Frequency & $175 \mathrm{MHz}$ & Peak field & $1.8 \mathrm{Kp}$ \\
\hline Voltage & $130 \rightarrow 101.2 \mathrm{kV}$ & Copper power & $683.9 \mathrm{~kW}$ \\
\hline Mean aperture $\left(\mathrm{R}_{0}\right)$ & $6.41 \rightarrow 5.16$ & Beam power & $613.1 \mathrm{~kW}$ \\
\hline Modulation $(\mathrm{m})$ & $1 . \rightarrow 1.6$ & Total power & $1297 \mathrm{~kW}$ \\
\hline
\end{tabular}

These IAP and Saclay estimates appear consistent with the RFQUIK estimate without the 0.6 fudge factor.

\section{Experience with Operating RFQs}

Various formulas are used to estimate the rf power requirements for an RFQ design. They tend to be more optimistic, that is to give a higher RFQ shunt impedance, Rs, than the values measured on operating RFQs. This is the case even including the "standard" assumption that measured Q will be 0.6-0.7 that of the SUPERFISH calculated Q. These formulas can be summarized as having the form $\mathrm{Rs}=\sim 10^{\wedge} 5 /$ (frequency, $\mathrm{MHz})^{\wedge}(\sim 1.5)$, but tending to give shunt impedances up to several times higher than $\mathrm{Rs}=10^{\wedge} 5 /(\mathrm{f}, \mathrm{MHz})^{\wedge}(3 / 2)$.

Shunt impedances for operating RFQs have been collected in the literature and from his experience with his own RFQs by Alwin Schempp, and updated by the author from the PAC, EPAC, and LINAC conference proceedings for 2000-2002, as shown in Fig. 6.4.1-2.

The relation $\mathrm{Rs}=10^{\wedge} 5 /(\mathrm{f}, \mathrm{MHz})^{\wedge}(3 / 2)$ is compared to the individual fits for 4-rod type RFQs ( $\left.R s=10^{\wedge} 4.09496 / f^{\wedge} 0.98306\right)$ and 4-vane-type RFQs (Rs=10^4.45071/f^1.241). It appears that 4-rod and 4-vane RFQs should be fitted separately. It is also clear that the experimental values should be used to help estimate the rf power that will be required in a new design.

There is a fairly large spread in the data, probably due to variations in construction technique that affected the achieved $\mathrm{Q}$ value for the structure. For example, the power 
required for the VE BERLIN 4-rod structure is almost twice the amount predicted for by the 4-rod-type fitted formula.

$($ rf power per meter, MW $)=1000(\text { vane voltage, MV })^{\wedge} 2 /(\mathrm{Rs}, \mathrm{k} \Omega \mathrm{m})$

\section{RFQ Shunt Impedance}

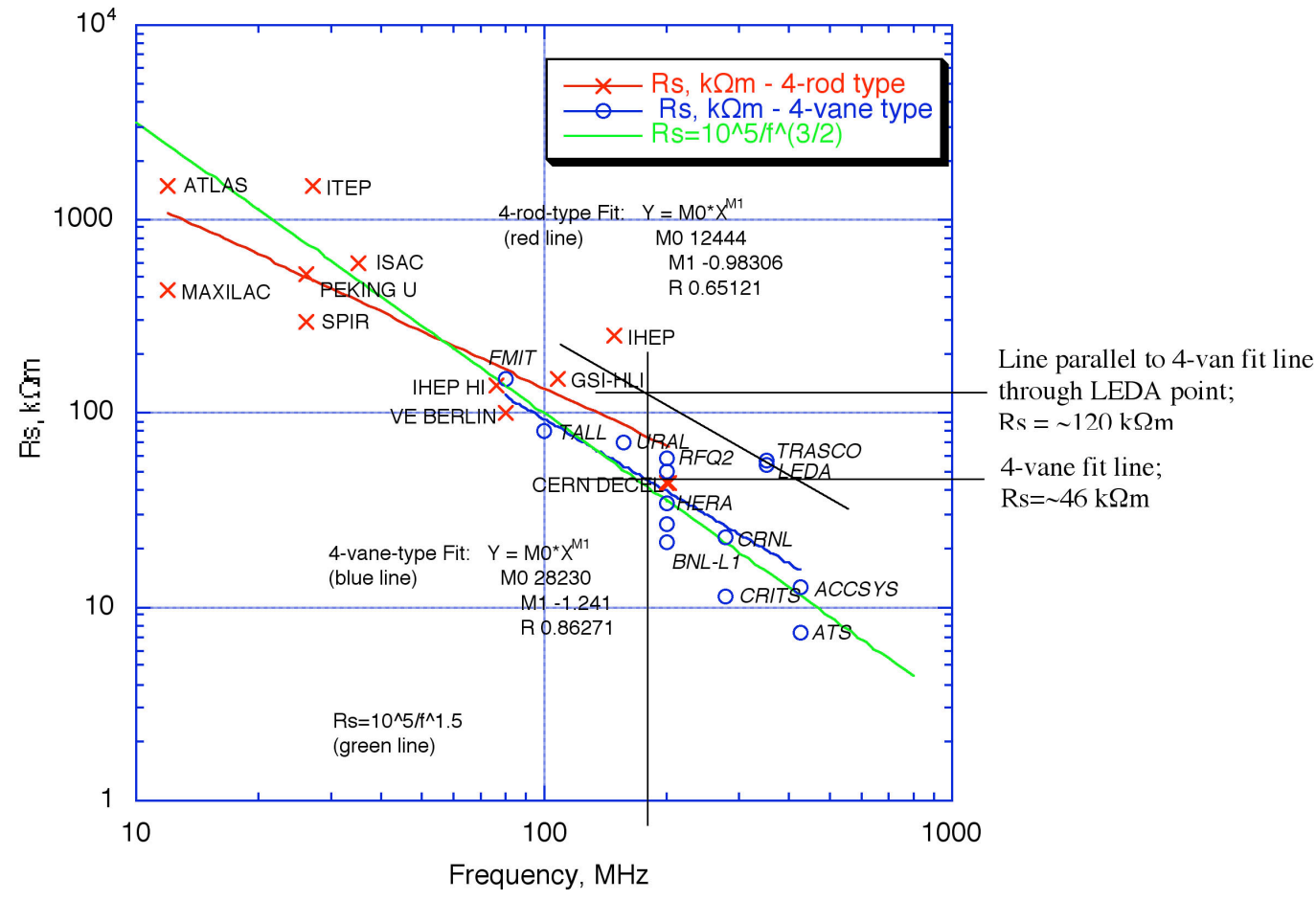

Fig. 7.2.1-2. Rf shunt impedance data from operating RFQs.

At $175 \mathrm{MHZ}$, the 4-vane fit gives Rs $=0.0460462 \mathrm{M} \Omega \mathrm{m}$.

Total Copper Power estimate using 4-vane fit $=2.86 \mathrm{MW}$

The LEDA achieved very good shunt impedance, and represents the RFQ most like IFMIFs. If we have the confidence to extrapolate from the LEDA data point back to 175 $\mathrm{MHz}$ using the slope of the 4-vane fit line, we get $\mathrm{Rs} \approx 0.120 \mathrm{M} \Omega \mathrm{m}$. This is close to the average for the Rs RFQUIK curve in Fig. 6.6.1-1.

Total Copper Power estimate using Rs $=0.120 \mathrm{M} \Omega \mathrm{m}=1.1 \mathrm{MW}$

\section{This is the copper power estimate used in Fig. 2.4-9,10.}

\section{Total Power}

Total $\mathrm{rf}$ power using the above estimates: RFQ copper power ranges from $0.684-2.86$ MW. Plus beam power, ranges from 1.3 to $3.5 \mathrm{MW}$. Assume $5 \%$ for rf transmission losses; range is 1.37 to $3.68 \mathrm{MW}$. Assume that the control margin can be absorbed in operational output ceiling of $680 \mathrm{~kW}$ (as assumed for the DTL) for the diacrode.

The CDR estimate states $3 \mathrm{MW}$. This is probably adequate. 


\section{Definitions, Questions, Comments}

This information was circulated to project participants for comment during preparation of the CDR; no comment was received; funding precluded further pursuit of the question with, for instance, the LEDA group.

The IFMIF Project needs further work on the RFQ power requirement.

\subsection{Vary Rho/rO Ratio?}

For a fixed vane voltage profile, Rho/r0 might be varied for lower peak field, as in the Alternative CDR RFQ. The resulting effect of the different multipole components on the beam would then have to be checked.

Similarly, if a Kilpatrick limit is used to set the vane voltage profile, a built-in Rho/r0 profile could be entered in LINACS and the design optimized on that basis.

There is so much flexibility in the parameter options that satisfactory beam performance might be found in combination with some advantage of a varying Rho/rO profile; the author has not explored this approach. 


\section{Simulation Codes}

Re-emphasizing the statement above: "A major requirement of the beam-based method is that the desired design performance be very closely verified by the detailed beam simulation. This was not lightly achieved, and required extensive development of the design method to include all of the effects to be simulated, and of the simulation code itself."

\subsection{The RFQ Design Code LINACSrfq}

The resulting design code is named "LINACS," of which "LINACSrfq" is a subset. It has also been used to design drift-tube linacs, and coupled-cavity linacs to $\sim 1 \mathrm{GeV}$ for radioactive waste transmutation and other purposes. The underlying space-charge physics section is applicable to any linac, requiring only the appropriate cell-by-cell bookkeeping and external field formulation to be applied. As an example of generality, a 2 -section linac with frequency jump between sections results in a long beam bunch at the entrance to the second section, which must then be shortened in a controlled way so there is similarity with the dc-to-bunched beam requirement in an RFQ.

The background is given in Section 5, Section 5.3 - Beam-Based Design Procedure, and accompanying references. The general procedure is apparent in Section 5.3.1 LINACSrfq Design Interface.

The maximum beam radius is determined from Sqrt[5]*(rms beam radius), assuming a uniform distribution. Using the subroutines of PARMTEQM $[19,20]$, the multipole terms are applied at this maximum radius, and the image-charge terms are applied at this maximum radius times the quadrupole flutter factor.

To achieve the required close correspondence to the simulation code results, it was necessary to include the effect of neighboring bunches in the LINACSrfq design code.

Optimization of the design proceeds as outlined in [7] and Section 5.3.3. Globally, the frequency is chosen, for which the global space-charge rule to limit the tune depressions to $\sim 0.4$ is useful.

To get started, the following default values should produce a reasonable first design, aided significantly by the use of the matched plus equipartitioned strategy:

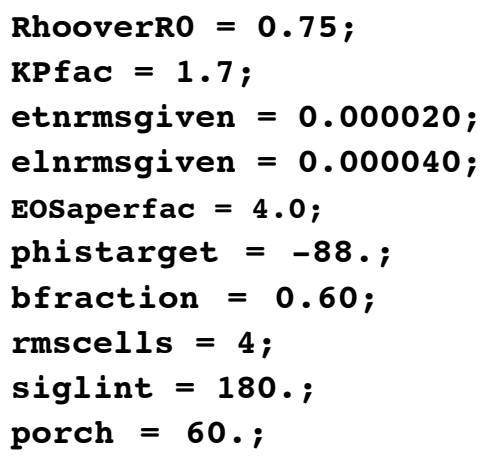




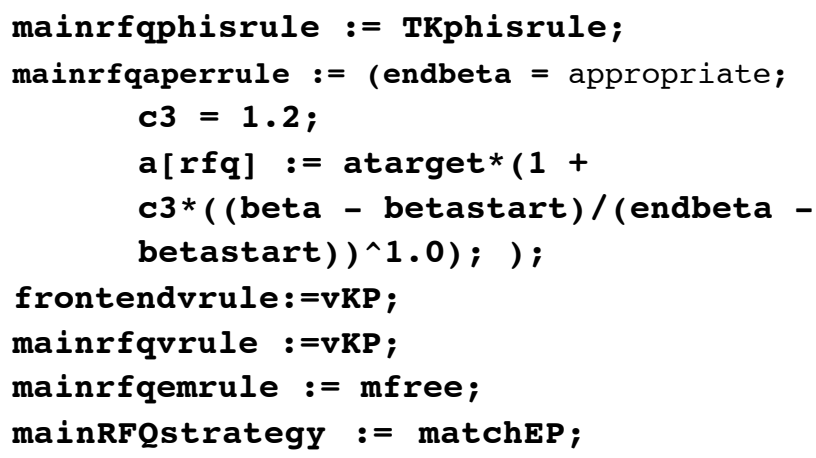

It is recommended that the strategy of bringing the beam to equipartition at the end of the shaper always be followed, as it always produces a reliable result. Many other strategies have been tested, such as the "conventional" one, but EP at EOS, along with the bunch formation strategy employed in the shaper, always gives good performance. The strategy for the main RFQ is then set as desired. The initial design is simulated in pteqHI, and a reasonable input match must be found.

The power and flexibility of Mathematica $\odot$ is very useful as a development platform; eventual reprogramming to obtain faster running speed would be useful for the optimization process.

An executable version is available to the project.

\subsection{The RFQ Simulation Code pteqHI}

\subsubsection{Development of pteqHI}

For the initial development of the RFQ at Los Alamos in 1978-1980, a simulation code named PARMTEQ was written based on the 2-term potential description of the RFQ fields. The RFQ description from the "conventional" design procedure provided a input table of the transverse focusing strength variable called $B$, the synchronous phase, the modulation and the voltage as a function of $z$ along the RFQ. The code was based on assumptions appropriate to the design requirements and computer capabilities of the time. For example, it used the position along the RFQ as the independent variable (resulting in non-canonical phase-space variables) and the paraxial approximation in the beam dynamics computation. The longitudinal phase-space is described by the phase and energy differences of each particle from the synchronous particle. Later, the higher-order field terms in the vane potential needed to describe the effects of the vane shape resulting from actual machining procedures were added, as well as many other features necessary to the final detailed design and manufacture of a practical RFQ, resulting in the widely used code PARMTEQM.

In developing a beam-based design procedure based on the envelope equations (and the equipartitioning equation), it became desirable to achieve a closer agreement between the design predictions from these equations and the simulation results given by PARMTEQM. Simply returning to the 2-term description did not suffice. It was also necessary to return to the more physical choice of time as the independent variable, as space-charge forces must be computed when the beam particles are at the same time. 
In a procedure where position is the independent variable, it is very difficult to accurately transform the particles from position to the same time, where the spacecharge computation is made, and then back again to the position, with the accuracy required to match the design requirement coming from the envelope equations. Also, and even more important for high-intensity machines where avoidance of beam loss is a crucial requirement, the details of beam loss are not handled accurately enough in the bunching and low energy portions of an accelerator (not only an RFQ) when position is used as the independent variable in the simulation code.

Therefore PARMTEQ was changed to a code named "pteqHI," first described in [18], using time as the independent variable for the natural computation of space-charge, eliminating the paraxial and other approximations, extending to enable simulation of multiple ion species and charge states simultaneously, and with many other options. The PARMTEQ $r-z$ mesh PIC method for the space-charge computation and the PARMTEQM analytic method (before the use of field maps) for multipole and image effects are incorporated. In the simple 2-term approximation mode but accurate timebased computation, pteqHI is also designed to be fast and efficient for optimization studies; however the execution time is presently much slower when the multipole and image effects are included. The source code is available.

Of course, all input and output pteqHI data files used for the results in this report are available to the project.

\subsubsection{Use of pteqHI}

Analysis of the cell-by-cell rms properties of the beam inside the RFQ is nonsense if particles are included that are already lost or in the process of being lost within the RFQ. PteqHI requires two full runs for full analysis. Particles lost within the RFQ are identified in the first run and flagged. In the second run, all the particles are simulated exactly as on the first run, but rms analysis is performed only on the particles that will be successfully transmitted to the RFQ output. This method has made it possible to reliably understand the space-charge physics within the RFQ and to develop the design code in exact correspondence.

Many options are available in pteqHI.

The code is structured to handle simultaneous distributions of ions; each distribution carries its own number of particles, input emittance data, charge, mass, current, and input energy.

The input distributions can be generated as usual from the input parameters in a number of analytical forms; typically a transverse waterbag and dc longitudinal for the $\mathrm{RFQ}$, or input particle distribution tables can be read in.

The RFQ cell parameters can be generated using the 2-term potential description or from multipole coefficient tables provided by LINACSrfq. Image-effect coefficient tables can also be provided by LINACSrfq.

The acceptance of the structure from the input (at any cell) to a downstream point can be measured. 
Options are available for the radial and longitudinal loss criteria.

Matrices of runs can be executed in batch mode, varying (for the first distribution listed) the input emittance alpha, beta or emittance, current, injection energy, and the RFQ vane voltage level by a vfac multiplying coefficient; in addition the beam current of two additional distributions can be varied.

Input and output beam transport lines can be added.

Input beam emittance match parameters can be found in a variety of ways.

- PARMTEQM assumes there is a match point just after the radial matching section, and uses an envelope equation method to compute backward to the RFQ input. This method yields an adequate match, but it can be considerably improved. PteqHI can iteratively use the beam itself from the input in to the match point, to minimize the difference between the specified and actual emittance alphas and betas at the match region. Either the same alpha and beta are found for the averaged $\mathrm{x}$ - and $\mathrm{y}$ - behavior, or separate $\mathrm{x}$ - and $\mathrm{y}$ - alphas and betas can be found.

- Other match strategies near the beginning of the RFQ have been tested, for example, minimization of the weighted standard deviation of beam size across a match region.

- The most reliable match is still found by a transmission matrix, found by running a batch matrix of input emittance alphas and betas (contamination by other beams can be included in the background). The transmission as a function of input ellipse parameters is often complicated [29] (Section 6.8), thus this matrix method is the most reliable.

At present, no graphics is done inside the code - in the development environment, the graphics requirement changed daily; it is easy to write output files and do the graphics separately.

Availability of the source code makes it easy to change or add other options.

\subsubsection{Beam Loss Criteria}

Particles in the RFQ are determined radially lost if they hit the vane surface. Some particles also travel between the vane tips out to significant radii. The inaccuracy of the rf fields outside the circle describing the innermost vane tip was discussed above; and assuming other factors (such as the space-charge routine) remain the same, the use of 3-D field maps and a criteria that a lost particle hits the vane surface is best. The accuracy of the 3D maps out to the appropriate particle radii must be established.

PteqHI uses the multipole and image field description of PARMTEQM as described above. A loss radial loss boundary that describes a typical vane-tip contour near the axis is generated using a quadrupolar function, as illustrated in Fig. 8.2-1. 


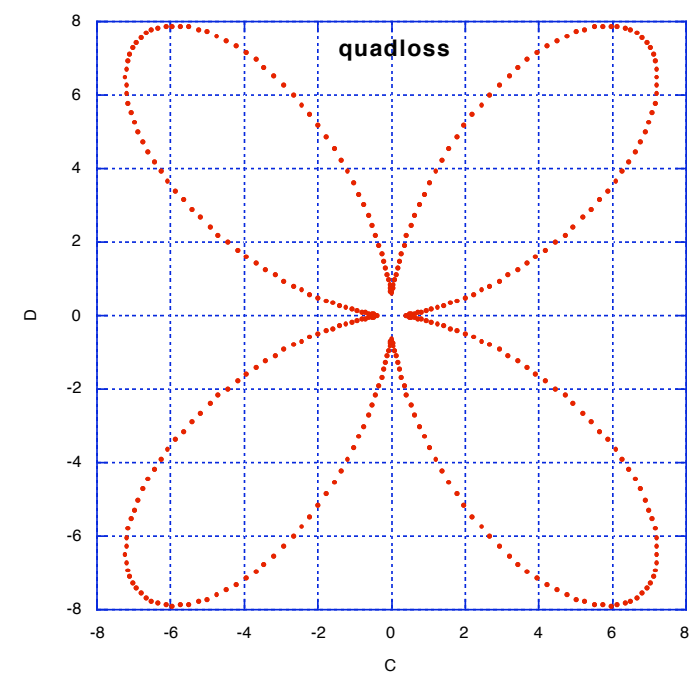

Fig. 8.2-1. Quadrupolar function with $+-10^{\circ}$ vanetip opening angle, computed at each step for $\mathrm{x}$ - and $\mathrm{y}$ - location of actual vane tips.

If a particle strays outward into the inter-vane region, the multipole fields rapidly become inaccurate and particles can receive very large energy kicks. A particle is declared radially lost if it hits the quadrupolar function boundary or a radius equal to 1.5 times the location of the outermost vane tip. The particle energy is inspected, and if it is greater than 1.25 times the synchronous energy, the synchronous energy is assigned as the energy of the lost particle.

A few particles can also receive non-realistically large energy kicks, or even have negative energy, although still within the radially accepted region; if this occurs, the particle is dropped from the simulation and its energy is assigned as the synchronous energy at that point. Later, these particles are also considered as radially lost at that position in the RFQ.

Finally, some particles may come nearly to rest and can cause the code to endlessly loop if not removed. Therefore a lower energy bound of 0.05 times the synchronous energy is set and particles are dropped from the simulation if their energy drops below this bound; their energy remains at the bound, and they are later considered as being lost radially at that position with that energy.

The loss criteria are applied at each time step of the simulation.

\subsubsection{Variation with Number of Particles simulated}

Tables 8.2-1,2,3 indicate the accelerated beam transmission at the output can be found with good accuracy even with $5-10 \mathrm{~K}$ simulated particles. The statistics for the total loss with energies above $1 \mathrm{MeV}$ are not good for less than 100K simulated particles. The computing time for $100 \mathrm{~K}-1 \mathrm{M}$ particles is still large, so such runs would be made only at the final design stage. 
Table 8.2-1. CDR RFQ - Variation of transmission with number of particles simulated.

\begin{tabular}{|l|l|l|l|l|l|}
\hline & $5 \mathrm{~K}$ WB & $10 \mathrm{~K}$ WB & $100 \mathrm{~K}$ WB & $1 \mathrm{M}$ WB & $\sim 1$ M Source Emit \\
\hline$\%$ losses $>1 \mathrm{MeV}$ & 0.0 & 0.01 & 0.034 & -- & 0.073 \\
\hline$\%$ Accelerated & 90.3 & 89.4 & 89.6 & -- & 89.4 \\
\hline
\end{tabular}

Table 8.2-2. AltCDR RFQ - Variation of transmission with number of particles simulated.

\begin{tabular}{|l|l|l|l|l|l|}
\hline & $5 \mathrm{~K}$ WB & $10 \mathrm{~K}$ WB & $100 \mathrm{~K}$ WB & $1 \mathrm{M}$ WB & $\sim 1$ M Source Emit \\
\hline$\%$ losses $>1 \mathrm{MeV}$ & 0.12 & 0.05 & 0.068 & & 0.123 \\
\hline$\%$ Accelerated & 91.2 & 91.3 & 90.9 & & 89.8 \\
\hline
\end{tabular}

Table 8.2-3. PostCDR RFQ - Variation of transmission with number of particles simulated.

\begin{tabular}{|l|l|l|l|l|l|}
\hline & $5 \mathrm{~K}$ WB & $10 \mathrm{~K}$ WB & $100 \mathrm{~K}$ WB & $1 \mathrm{M}$ WB & 1M Source Emit \\
\hline$\%$ losses $>1 \mathrm{MeV}$ & 0.02 & 0.08 & 0.077 & 0.075 & 0.081 \\
\hline$\%$ Accelerated & 97.0 & 97.1 & 97.1 & 97.1 & 95.8 \\
\hline
\end{tabular}

Tables 8.2-1,2,3 Percent of particles accelerated and \% losses with energies above $1 \mathrm{MeV}$, for ideal waterbag input distributions with $5 \mathrm{~K}, 10 \mathrm{~K}, 100 \mathrm{~K}$ and $1 \mathrm{M}$ particles, and for the ion source emittance distribution of $\sim 1 \mathrm{M}$ particles rms matched to the RFQ input.
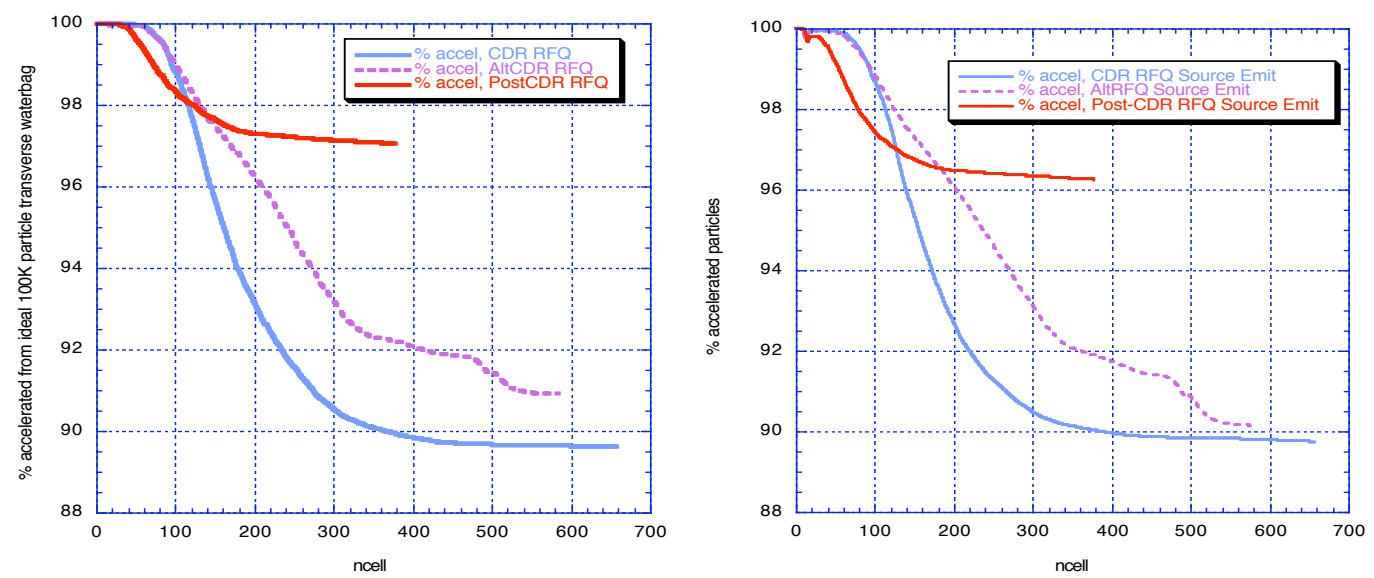

Fig. 8.2.2. Left - \% accelerated particles, $100 \mathrm{~K}$ waterbag initial distribution; Right - \% accelerated particles, Source Emittance initial distribution. 
Fig. 8.2.2 shows the cell-by-cell transmission of the three RFQs for the $100 \mathrm{~K}$ waterbag and Source Emittance distributions.

Figs. 8.2.3-5 show, for the Post-CDR RFQ, the variation in the \% of all lost particles vs. energy and vs. cell number as a function of the number of particles simulated.

Fig. 6.2.6 shows the matching sensitivity results for all three RFQs for $100 \mathrm{~K}$ particles (to the left) and $10 \mathrm{~K}$ particles (to the right). The trends are observable with $10 \mathrm{~K}$ particles; the statistics are only adequate at $100 \mathrm{~K}$ particles, but at present the computer runs take a long time even with $100 \mathrm{~K}$ particles. The figures were generated on a grid of 7 alphas and 5 beta. A Mathematica (C) interpolation polynomial, which leaves the values unchanged at the given grid points, was used to fill in the mesh by a factor of five in each direction.

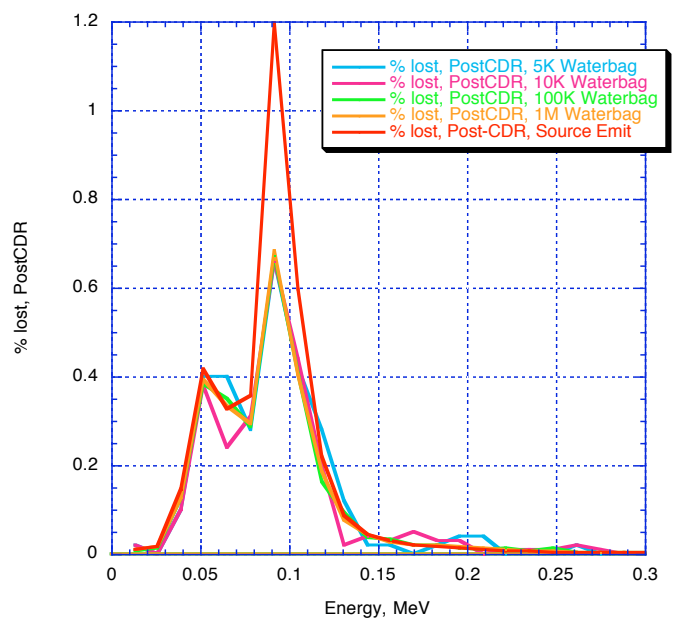

Fig. 8.2-3. Post-CDR RFQ. \% of all lost particles vs. energy where lost, $0.0-0.3$ $\mathrm{MeV}$.
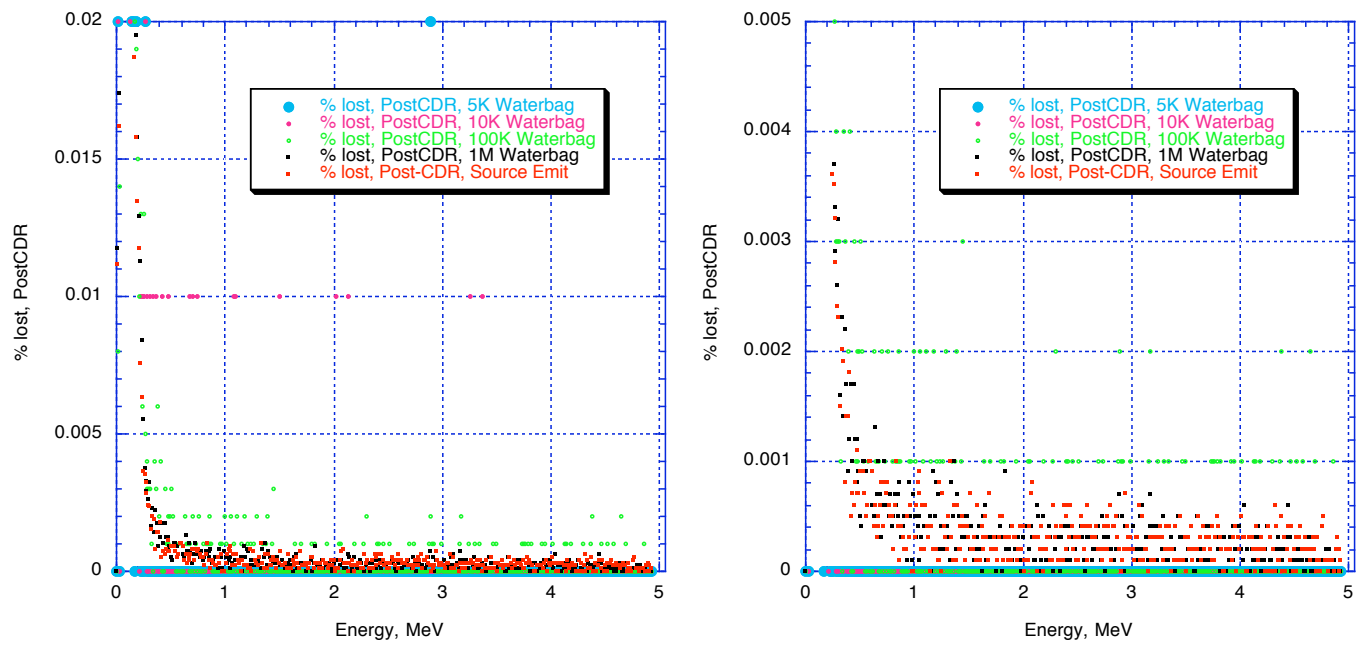
Fig. 8.2-4a,b. Post-CDR RFQ. \% of all lost particles vs. energy where lost, expanded vertical scales. For $5 \mathrm{~K}, 10 \mathrm{~K}, 100 \mathrm{~K}, 1 \mathrm{M}$ particles, loss of 1 particle is $0.02 \%, 0.01 \%$, $0.001 \%, 0.0001 \%$. (400 bins, each $5.05 \mathrm{MeV} / 400=0.012625 \mathrm{MeV}$ ).

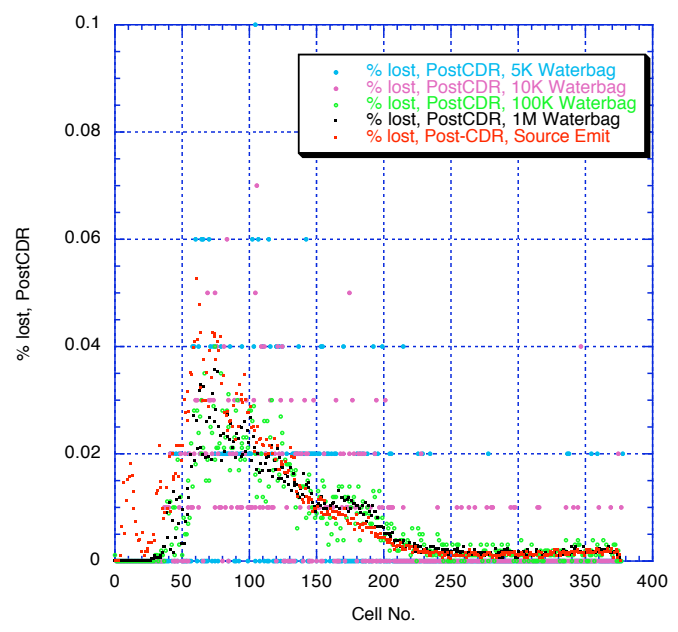

Fig. 8.2-5. Post-CDR RFQ. \% of all lost particles vs. position ( $z$, meters) where lost. CDR.

Fig. 8.2.6. Left with $100 \mathrm{~K}$ particles, right with $10 \mathrm{~K}$ particles.
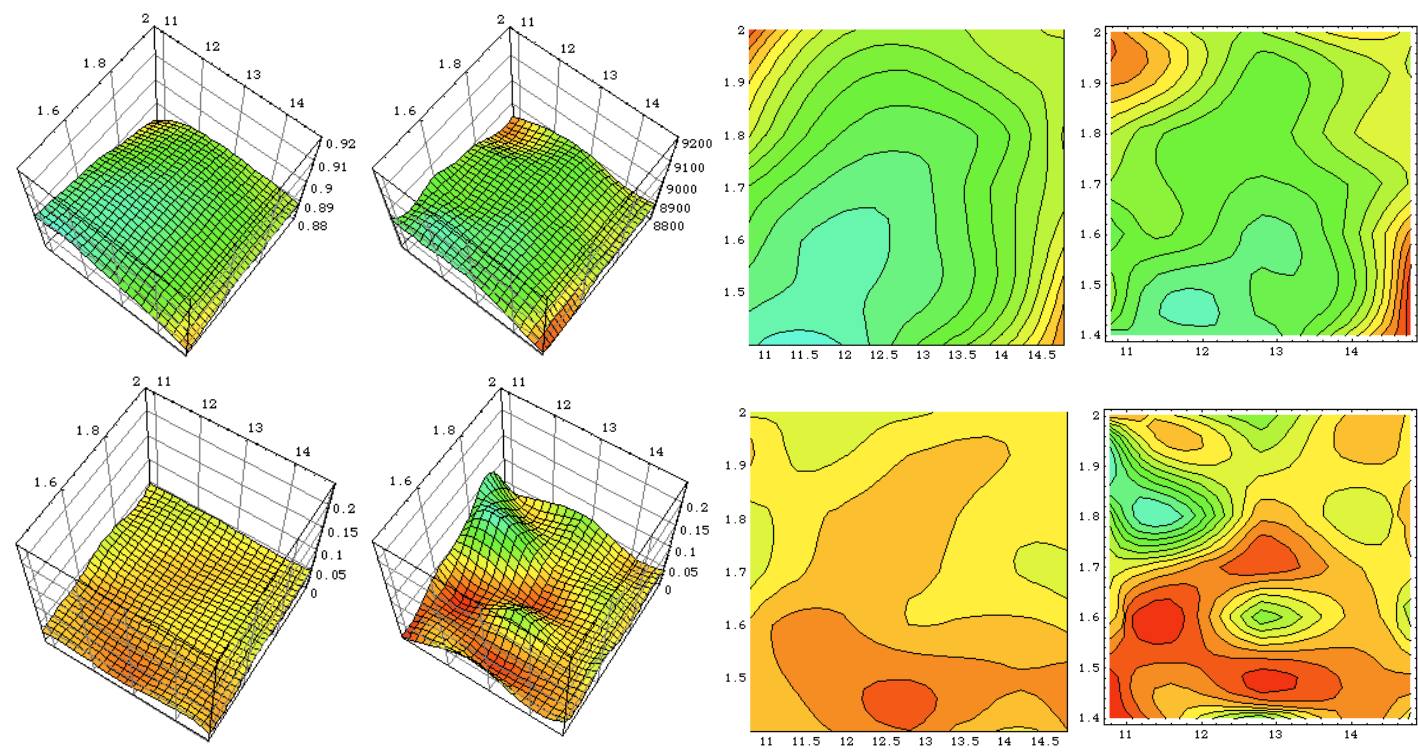


\section{AltCDR}
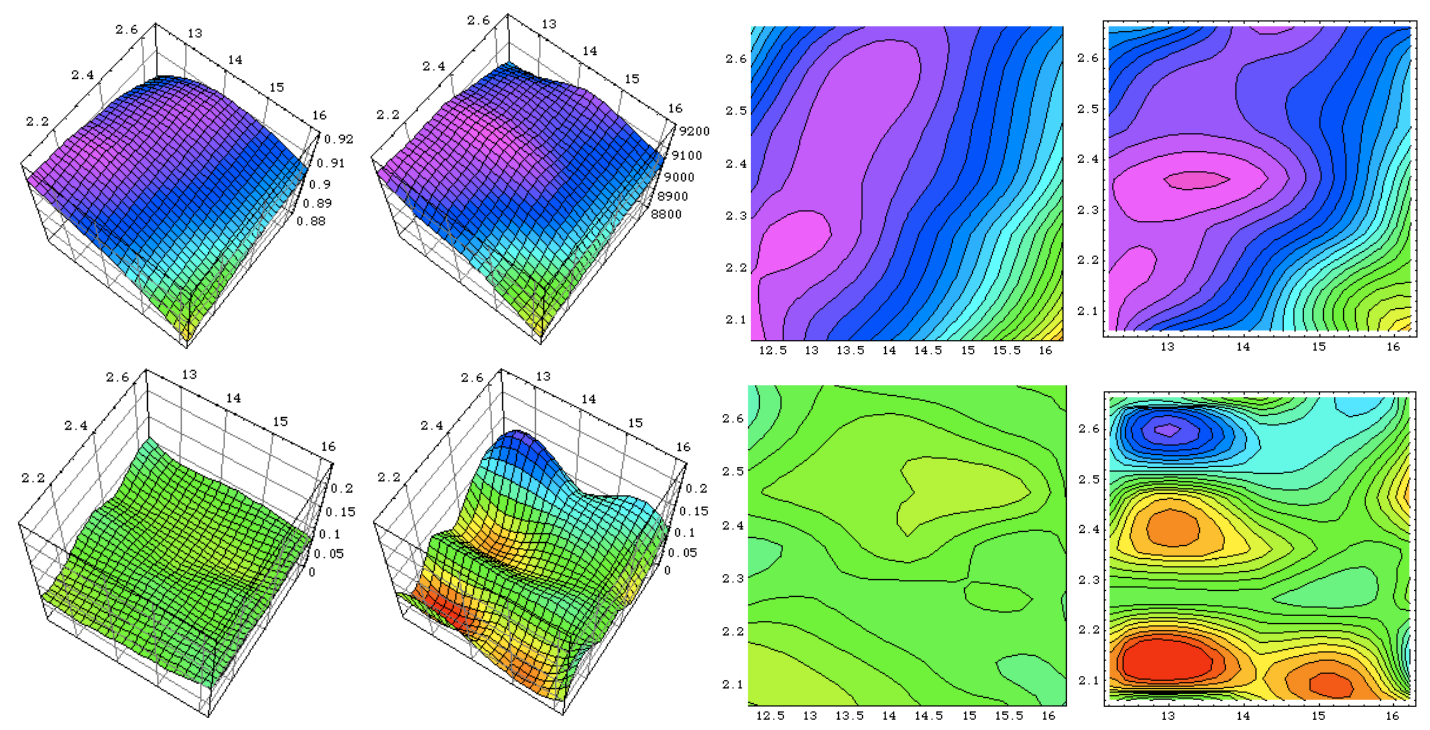

PostCDR
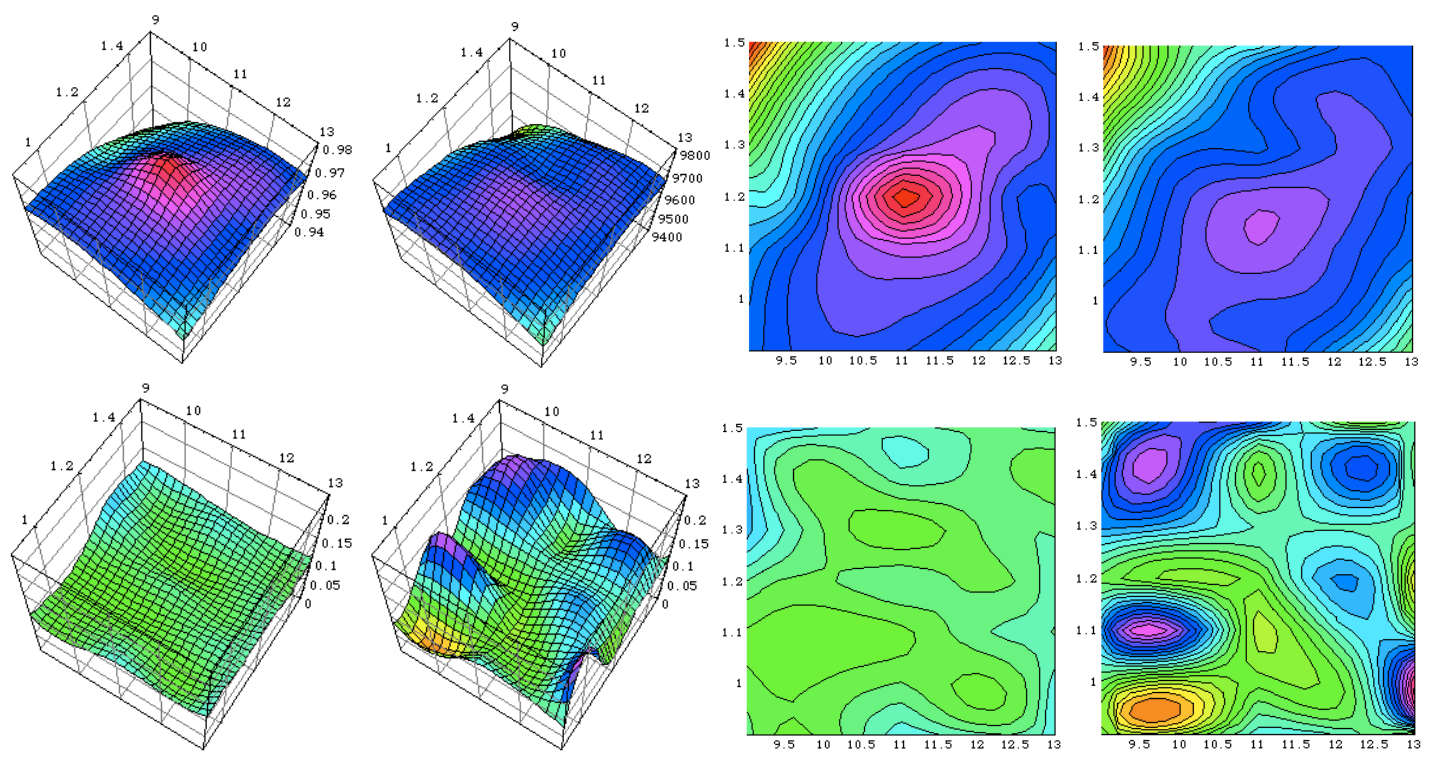


\subsection{Other RFQ Simulation Codes}

Comparison to PARMTEQM (versions in which the paraxial approximation has been removed) show that the overall transmission may be comparable within a few percent, but that the loss patterns (the distribution and energy of loss particles) differ significantly. This difference has also been observed in the LEDA RFQ, the most powerful and longest $\mathrm{cw} R \mathrm{RQ}$ to date [12,21]. It has not been possible to compare the LEDA data with a pteqHI simulation; this would be a valuable exercise.

Other groups also made new codes that grew from PARMTEQM but removed many of the assumptions to achieve better accuracy. These codes used time as the independent variable, removed the paraxial approximation, and replace the vane potential description formulas by full 3-D field maps exactly conforming to the metal shape as it would be manufactured. LIDOS, by the Moscow Radiotechnical Institute, is fully developed and available commercially, including the source code. The TOUTATIS [24] code was derived from PARMTEQM with removal of certain approximations, especially the paraxial, and with 3-D field maps; the reference contains an excellent comparison of the fields produced in the vane-tip region by PARMTEQM and by the TOUTATIS 3-D field map method. The PARMTEQM (pteqHI, etc.) analytically expressed fields are really accurate only in the circle describing the innermost vane-tip, and can have large local errors outside this region. (The latest PARMTEQM version has 3D field maps, but unfortunately still has inaccurate space-charge computation by retaining position as the independent variable.) Beam loss results from the analytical method have been compared to LIDOS [50] and TOUTATIS; the overall transmission results are usually within a few percent; detailed comparison and analysis of the beam loss patterns have not been done. A number of other codes are also being developed independently.

The space-charge simulation method itself (assuming that the dynamics correctly assigns the canonical time coordinates to the particles at the space-charge computation point) is central to many details of the beam-loss pattern. PteqHI, PARMTEQM and TOUTATIS all use the same underlying $r-z$ mesh PIC method, which is fundamentally reliable, but which has many details that affect the space-charge computation. Absolute results are subject to error bars, whose magnitude is extremely difficult to determine because of uncertainties in the physical and computational modeling. Relative comparisons between designs might be expected to be trustworthy; however, there is disconcerting evidence when comparing different codes even on this point, and detailed comparison of source code is needed.

Source code availability is essential for a project to be able to make informed decisions about the final modeling for the project. PteqHI and LIDOS source code are available.

The IFMIF project expended a large fraction of the limited resources available in 2004 trying to use the TOUTATIS code to reproduce published results and then work beyond toward improved RFQ designs. An executable version was available and a single input datafile was furnished from which the results had been obtained. Execution did not reproduce the expected transmission. Questions were posed and help requested; finally a petition was made to the code author, who kindly suggested different setting of various options in the input file. Unfortunately, the results were still always less than 
the referenced result, and the many other questions were not answered. Therefore official use by the Project was discontinued, until the code is more fully developed, and most important - until source code would be made available from which independent judgments can be made.

The exact transmission found by any of these codes should be treated as indicative, as each has various sensitivities in its internal methods. Continued work to compare results between different codes more broadly and deeply than heretofore is highly recommended - this requires comparison at source code level and devising of appropriate tests. It is strongly recommended that the project obtain source code for all important simulation work. 


\section{Ion-Source/LEBT Input Beam Modeling}

A few notes on the ion source/LEBT:

\section{ECR Ion-Source Status}

There was substantial concern during the early years of the IFMIF program about the reliability of an IFMIF ion source. IFMIF provided support for a thorough engineering evaluation of the ECR ion source. Several runs accumulating several 1000 hours of $\mathrm{cw}$ operation at $\sim 100 \mathrm{~mA}$ protons showed that the availability of the ECR source should be high.

The $140 \mathrm{~mA}$ deuteron beam performance within the specified emittance has not been demonstrated, either with deuterons or with $\sim 200 \mathrm{~mA}$ protons to investigate a scaled result. See Section 1.1.1.

List Cont ourPlot [da taheight, PlotRange $\rightarrow\{-0.001,1\}$, Contour $\rightarrow 50$.
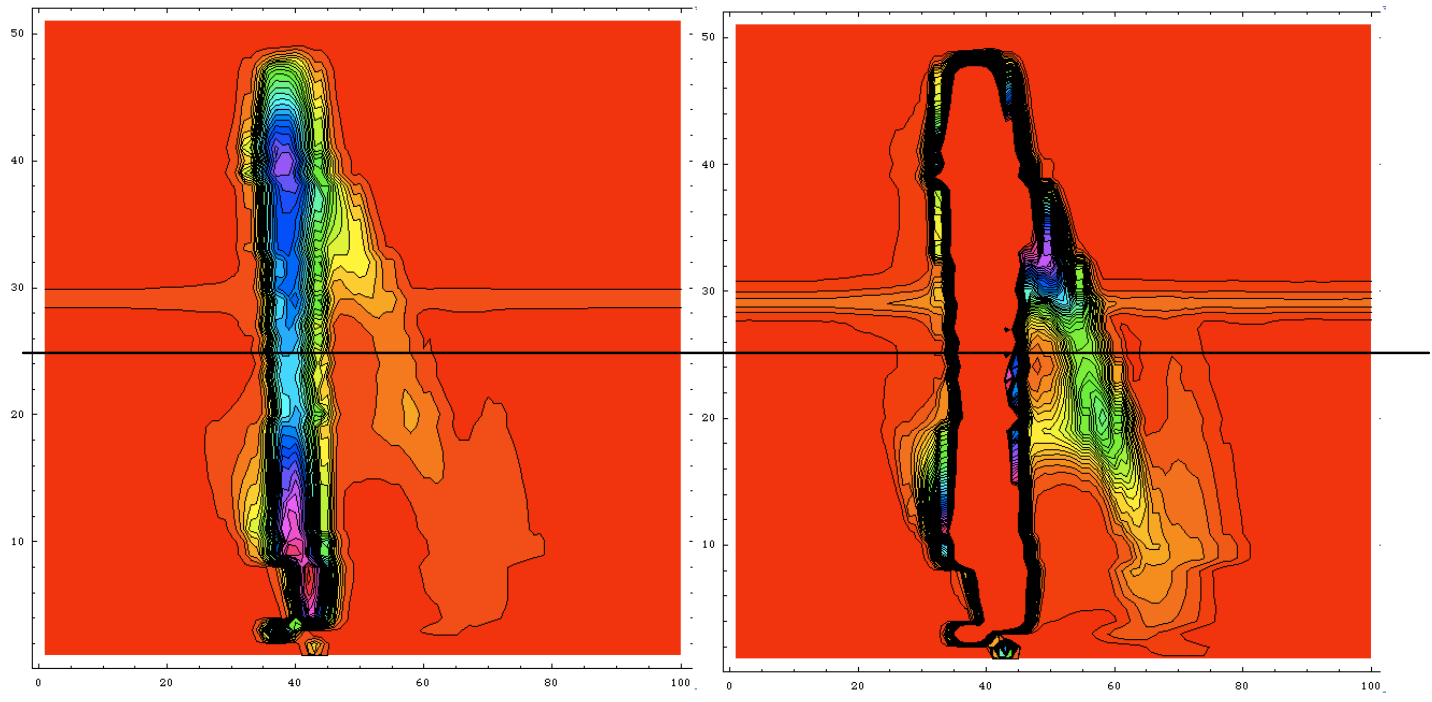

ListPlot [da taheight [[25]].

PlotJoined $\rightarrow$ True, PlotRange $\rightarrow$ Al1]

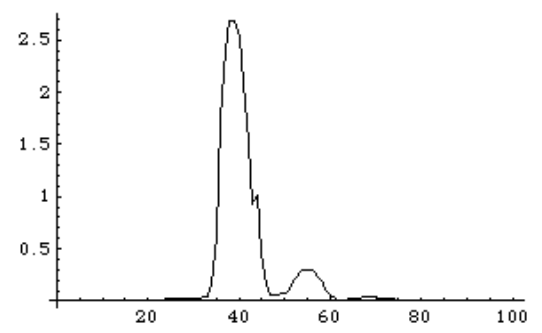

Cross-section at ordinate value $=25$.

Fig. 9.1 Raw Data Characteristics of the ECR Ion-Source Emittance Distribution (see figures, Section 6.6) Right figure has expanded vertical scale.

As noted in Section 1.1.1, this result for $\sim 100 \mathrm{~mA}$ protons is the only emittance data set available to the project. The halo appears to be composed of protons. The data set is 
thresholded by an inaccurate (and optimistic) method. The more accurate method developed by M. Stockli at SNS should be used. For this report, the data set has been numerically transformed to the input $\alpha$ and $\beta$ ellipse matching parameters required by the RFQs. The rms value is also adjusted to equal that of the ideal waterbag distribution.

\section{CDA Section 2.6.2.10.1 Injector beam}

The IGUN output is converted to a particle input appropriate for the TOPKARK code, which is used to model beam dynamics in a two-solenoid, space-charge-compensated LEBT. The beam radius is kept to less than half the 6-cm radius of the $15 \mathrm{~cm}$ long solenoids, which have a peak on-axis magnetic field of $0.55 \mathrm{~T}$. The fringe fields are modeled using an analytical expression that agrees well with measurements of solenoids encased in an iron shield. Roughly $30 \%$ of the halo (3\% of the beam) is scraped inside the solenoids. The background neutral gas in the LEBT is assumed to provide $98 \%$ space charge neutralization, implying an effective current of $3 \mathrm{~mA}$. Fluctuations in the source current of $\pm 1 \%$ on a time scale faster than the neutralization time are assumed to vary the effective current from $1.5 \mathrm{~mA}$ to $4.5 \mathrm{~mA}$, which leads to a time-varying beam mismatch at the RFQ entrance. Although this process is not fully understood and is difficult to model, the effect is approximated by overlapping the final particle distribution from three separate simulations using the minimum, average and maximum effective current. This results in an effective RMS emittance growth of $33 \%$. Aberrations due to nonlinear magnetic fields and space charge forces are small compared to this mismatch effect.

\section{LEDA LEBT}

An electron ring is needed just before the RFQ input, to compensate for the short unneutralized section between the end of the LEBT and the beginning of the RFQ vanes. (LYoung, LINAC’2000, [33])

CDA LEBT - CDA Section 2.6.2.2, Fig. 2.6.2-3

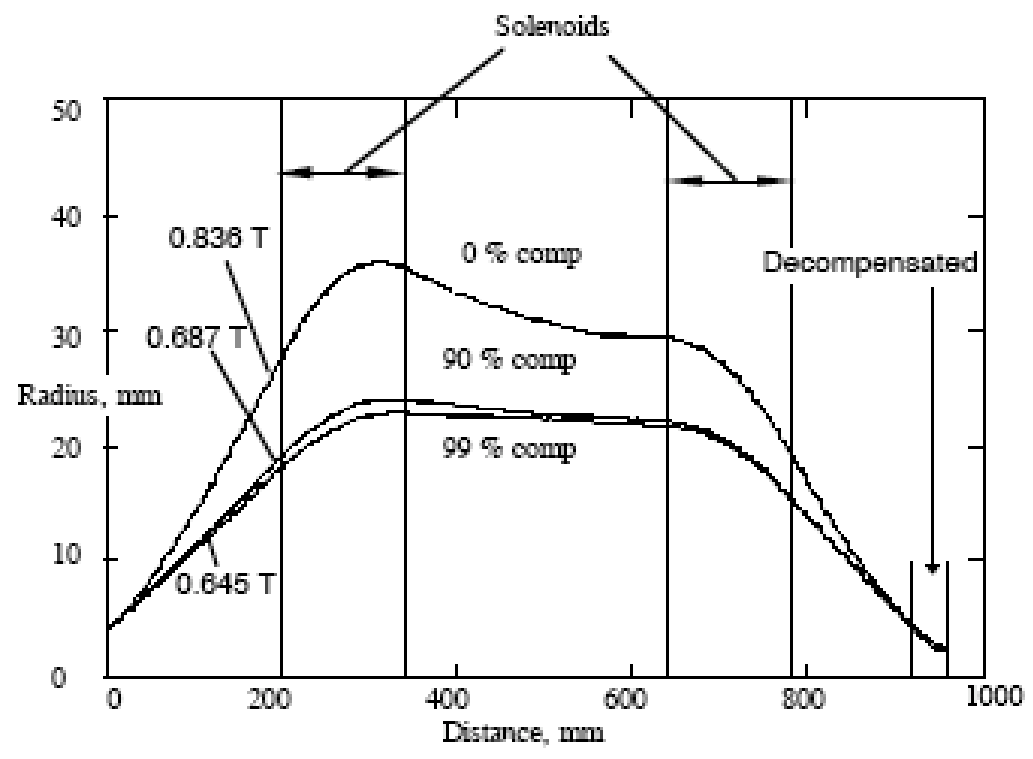

Figure 2.6.2-3. Effect of charge compensation ce bexm radius in a solenoidal LEBT.

Fig. 9.2 LEBT outlined in the CDA 


\section{From an IAP Report.}

Extensive new work has been performed by IAP in 2006 under this same EFDA Work Package.

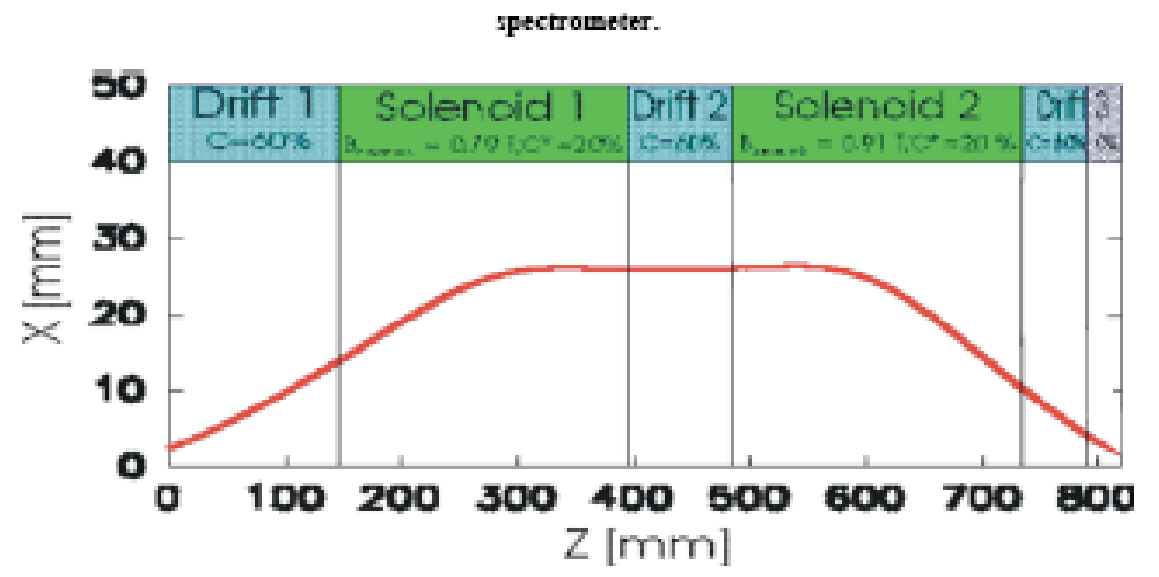

Figure 7: Schematic dranimg of the magnetic LEBT for IFMIIF including beam eavelope for $140 \mathrm{~mA}$.

Fig. 9.3 A representative LEBT outlined by IAP

\section{SILHI LEBT}

/Users/rajameson/Documents/Folders/IFMIF/ ACC \IS:LEBT/IFMIF \ $\mathrm{BLM} / \mathrm{ECR} \backslash \& L E B T / E m M s m t$ SaclECRLEBT . bmp

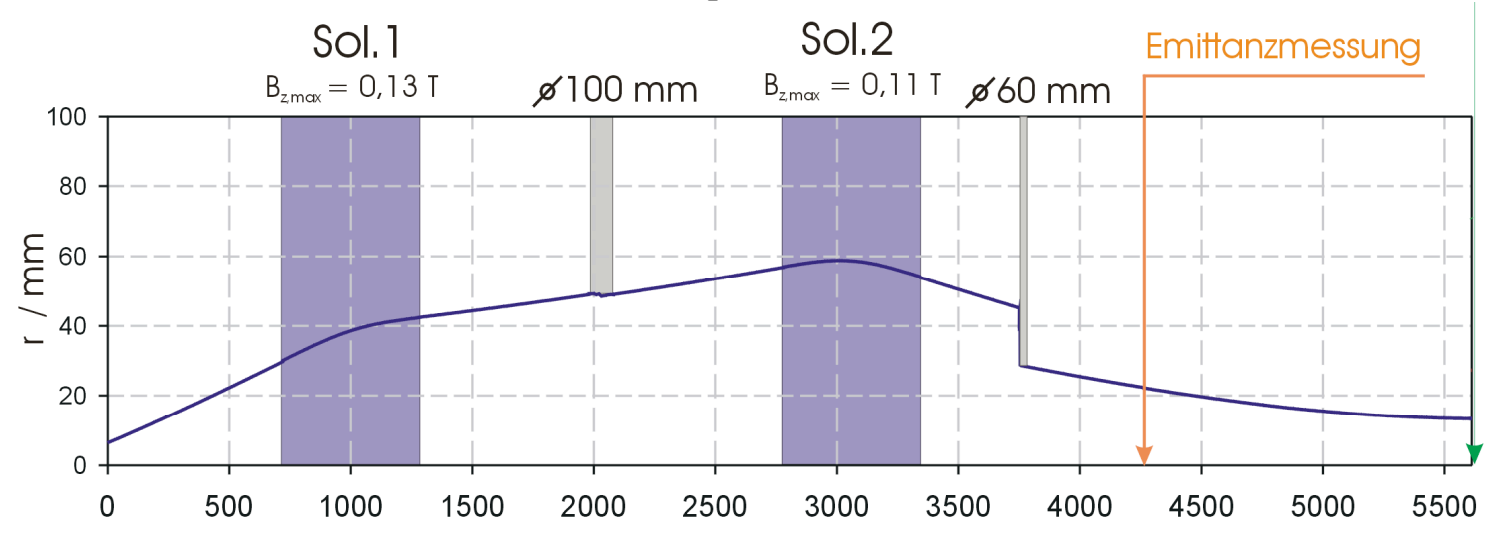

Fig. 9.4 A LEBT considered by Saclay includes a collimator.

A collimator in the LEBT (also used at SNS) may be helpful for scraping halo beam. 


\section{Concluding Remarks}

Summary observations and comments are given in Section 6.9.

The careful reader will have noticed various features, such as some oscillation of the "matched" rms beam profiles. These are the subject of ongoing research, involving basic issues such as adiabaticity, phase-space mixing and so on. There is also subtle evidence concerning the role of adiabaticity in the optimization process. The RFQ remains a very interesting example of general linear accelerator physics.

The simulations reported were performed with a consistent family of codes. Relevant comparison with other codes has not been possible as their source code is not available. Certain differences have been noted but are not consistent over a broad range of designs and parameter range. The exact transmission found by any of these codes should be treated as indicative, as each has various sensitivities in its internal methods. Continued work to compare results between different codes more broadly and deeply than heretofore is highly recommended - this requires comparison at source code level and devising of appropriate tests. It is strongly recommended that the project obtain source code for all important simulation work. 


\section{Acknowledgement}

"Vielen herzlichen Dank" to Professor Dr. H. Klein for his reading of the manuscript and his useful comments. 


\section{References}

1 A.L. Trego et al., "Fusion Materials Irradiation Test Facility - A facility for Fusion Materials Qualification", Nuclear Technology/Fusion 4(2), 695 (1983).

2 K. Noda et al., "Capability of energy selective neutron irradiation test facility (ESNIT) for fusion reactor materials testing and the status of ESNIT program", Journal of Nuclear Materials 191194, 1367 (1992).

3 IFMIF Comprehensive Design Report, IFMIF International Team, An Activity of the International Energy Agency (IEA) Implementing Agreement for a Program of Research and Development on Fusion Materials, January 2004.

4 K. R. Crandall et al., "RF Quadrupole Beam Dynamics Design Studies," Proceedings of the 1979 Linear Accelerator Conference (Brookhaven National Laboratory, Upton, New York, 1980) BNL-51134, p. 205.

5 T. Wangler, "RF Linear Accelerators," Wiley Series in Beams Physics and Accelerators Technology.

6 L. Young, "25 Years of Technical Advances in RFQ Accelerators," MOPPB001, PAC 2003.

7 IFMIF Comprehensive Design Report, IFMIF International Team, An Activity of the International Energy Agency (IEA) Implementing Agreement for a Program of Research and Development on Fusion Materials, January 2004. RFQ design based on R. A. Jameson, "Some Characteristics of IFMIF RFQ KP1.7 Designs," IFMIF Memorandum RAJ-24-May-2000, Revised 6 September 2000. Included as Appendix 1.

8 Email from R. A. Jameson, IFMIF Accelerator Facility Design Team Leader, to Distribution, Subject: "AccFac CDR Draft 3 June 2003," Date: Tuesday, June 3, 2003, 4:01 PM.

9 IFMIF CDA, Section 2.6.2.10, Beam dynamics, beam performance and beam loss simulations; Injector and RFQ modeling by D. Bruhwiler.

10 M. Stockli, file: 'stockli emit meas.pdf,' title: 'Microsoft PowerPoint - stockli_final_for_cd.ppt,' 9/25/2002, 9:20 pm, detailed presentation to the SNS ASAC, September 2002, "SelfConsistent, Unbiased Exclusion Methods of Emittance Analysis," PAC 2004.

11 "IFMIF Accelerator Technical Memorandum 3-10-96: LEBT Material, R. A. Jameson, AOT1:96-078, Los Alamos National Laboratory, 10 March 1996.

12 "Record of discussions about the Accelerator Production of Tritium (APT) Low Energy Demonstration Accelerator (LEDA) Radio Frequency Quadrupole RFQ Discussion at LANL on 1/14/2000 with Lloyd Young and Dave Schneider," IFMIF Memorandum, R. A. Jameson, 8 February 2000.

13 "Coulomb Scattering of Beam Particles on Residual Gas: A Source of the Halo at Low Energy," N. Pichoff, G. Haouat, and P.-Y. Beauvais.

14 KEP Report, 3.1.2 AC11B-EU-IAP, Long run test of ion source.

15 R. A. Jameson et al., "Scaling and Optimization in High-Intensity Linear Accelerators," LACP-91-272, Los Alamos National Laboratory, July 1991 (introduction of LINACS design code).

16 R. A. Jameson, "On Scaling and Optimization of High Intensity, Low-Beam-Loss RF Linacs for Neutron Source Drivers," AIP Conference Proceedings 279 (1992), p. 969.

17 R. A. Jameson, "Beam-Intensity Limitations in Linear Accelerators," (Invited), Proceedings of the 1981 Particle Accelerator Conference, Washington, D.C., March 11-13, 1981, IEEE Trans. Nucl. Sci. 28, p. 2408, June 1981; Los Alamos National Laboratory Report LA-UR-81-765, 9 March 1981. Correction, Jameson, R. A.; IEEE Trans. Nucl. Sci.; 1981; Vol. 28, No. 4, pp. 3665-3665. 
18 R. A. Jameson, "A Discussion of RFQ Linac Simulation," Los Alamos National Laboratory Report LA-CP-97-54, September 1997.

19 K. R. Crandall, "Effects of Vane-Tip Geometry on the Electric Fields in Radio-Frequency Quadrupole Linacs," Los Alamos National Laboratory, LA-9695-MS, April 1983.

20 K. R. Crandall, "PARMTEQ with Image Charges," Los Alamos National Laboratory, AT-1-92213, October 1991.

21 L. Young, "Simulations of the LEDA RFQ 6.7 MeV Accelerator," PAC 1997.

22 B. Bondarev et al., "The Lidos RFQ Designer Development," Proceedings of the 2001 Particle Accelerator Conference, Chicago.

23 R. Duperrier and R. Ferdinand, "TOUTATIS, THE CEA-Saclay RFQ Code," XX International Linac Conference, Monterey, California.

24 R. Duperrier, "Intense Beam dynamics in RFQs," Thesis, U. Paris XI Orsay, Orsay Serial No. 6194, 7 July 2000.

25 V. A. Teplyakov, "The First CW Accelerator in USSR and a Birth of Accelerating Field Focusing," EPAC 2006, Edinburgh, June 2006.

26 R. Ferdinand, "How to Chose the Operating Point," Pac 1995, Vol. 2, p. 1146.

27 "Optimization of RFQ Design," R. Ferdinand et al., EPAC 1998, THP23G.

28 R. A. Jameson, "Self-Consistent Beam Halo Studies and Halo Diagnostic Development in a Continuous Linear Focusing Channel," LA-UR-94-3753, Los Alamos National Laboratory, 9 November 1994. AIP Proceedings of the 1994 Joint US-CERN-Japan International School on Frontiers of Accelerator Technology, Maui, Hawaii, USA, 3-9 November 1994, World Scientific, ISBN 981-02-2537-7, pp. 530-560.

29 "RFQ Optimization Study for ESNIT," H. Takeda and R. A. Jameson, Los Alamos National Laboratory Report LA-CP-93-5, "Deuteron Linac Design Aspects for ESNIT," pp. 4-25, January 1993.

30 A. Schempp, Proceedings of EPAC88, 1989, p 464.

31 A. Schempp, Proceedings of PAC89, IEEE89, CH2669-0, 1989, p. 1093.

32 S. Yamada, "Buncher section optimization of heavy ion RFQ linac," Proceedings of the 1981 Linear Accelerator Conference.

33 L. Young, "High Power Operations of LEDA," LINAC 2000.

34 L. Young, "Operations of the LEDA Resonantly Coupled RFQ," WOAA004, PAC 2001, Snowmass.

35 F. Sacherer, "RMS envelope equations with space charge, CERN Internal Report, SI-DL/7012.

36 R. A. Jameson, 1993 Particle Accelerator Conference, Washington, D.C., 17-20 May 1993, IEEE Conference Proceedings, IEEE Cat. No. 93CH3279-7, 88-647453, ISBN 0-7803-1203-1.

37 R. A. Jameson, "Beam Halo from Collective Core/Single-Particle Interactions, Los Alamos National Laboratory Report LA-UR-93-1209, March 1993.

38 R. A. Jameson, "An Approach to Fundamental Study of Beam Loss Minimization," AIP Conference Proceedings 480, "Space Charge Dominated Beam Physics for Heavy Ion Fusion," Saitama, Japan, December 1998, Y. K. Batygin, Editor.

39 I. Hofmann,, "Emittance Growth of Beams Close to the Space Charge Limit," 1981 PAC, IEEE Trans. Nucl. Sci., Vol. NS-28, No. 3, June 1981, p. 2399, I. Hofmann and I. Bozsik, "Computer Simulation of Longitudinal-Transverse Space Charge Effects in Bunched Beams," 1981 Linac Conference, October 1981, LA-9234-C, Los Alamos National Laboratory, February 1982, p. 116.

40 R. A. Jameson, "Equipartitioning in Linear Accelerators," Proceedings of the 1981 Linear Accelerator Conference, Santa Fe, New Mexico, October 19-23, 1981, Los Alamos National 
Laboratory Report LA-9234-C, p. 125, February 1982; Los Alamos National Laboratory Report LA-UR-81-3073, 19 October 1981.

41 R. A. Jameson, "On Scaling and Optimization of High Intensity, Low-Beam-Loss RF Linacs for Neutron Source Drivers," AIP Conference Proceedings 279, ISBN 1-56396-191-1, DOE Conf-9206193 (1992) pp. 969-998, Proceedings of the Third Workshop on Advanced Accelerator Concepts, 14-20 June 1992, Port Jefferson, Long Island, New York, LA-UR-922474, Los Alamos National Laboratory, 28 July 1992.

42 R. A. Jameson, "An Approach to Fundamental Study of Beam Loss Minimization," AIP Conference Proceedings 480, "Space Charge Dominated Beam Physics for Heavy Ion Fusion," Saitama, Japan, December 1998, Y. K. Batygin, Editor. Workshop on Space Charge Dominated Beam Physics for Heavy Ion , 10-12 December 1998, Institute of Physical and Chemical Research (RIKEN), Wako-shi, Japan. Los Alamos National Laboratory Report LAUR-99-129, 8 January 1999.

43 "Review of Beam Dynamics and Space Charge Resonances in High Intensity Linacs," I. Hofmann et al., EPAC 2002.

44 C. Zhang, Z. Y. Guo, A. Schempp, R. A. Jameson, J. E. Chen, and J. X. Fang, Low-beam-loss design of a compact, high-current deuteron radio frequency quadrupole accelerator, Phys. Rev. Special topics - Accelerators and Beams, Vol. 7, 100101 (2004).

45 L. Young (1/21/2006), A. Schempp (2006), and V. A. Teplyakov (2006); private communications.

46 J. Rathke, private communication (1/24/2006).

47 "IFMIF Conceptual Design Activity, Final Report", IFMIF-CDA Team (ed. by M. Martone), ENEA Frascati Report, RT/ERG/FUS/96/11, December 1996.

48 Accelerator Team Meeting for the IFMIF Conceptual Design Activity, Santa Fe, New Mexico, USA, 11-13 September 1995, Los Alamos National Laboratory Report LA-UR-95-4416.

49 R. A. Jameson, "RF Power Estimate for IFMIF CDR RFQ," IFMIF Memorandum IFMIF-RAJ030603-DRAFT.

50 Report "IFMIF RFQ Investigations," International Science and Technology Center Moscow Radiotechnical Institute, Computer Codes For High Power Linacs, Project \#2135p, Moscow 2003. 


\section{Appendix}

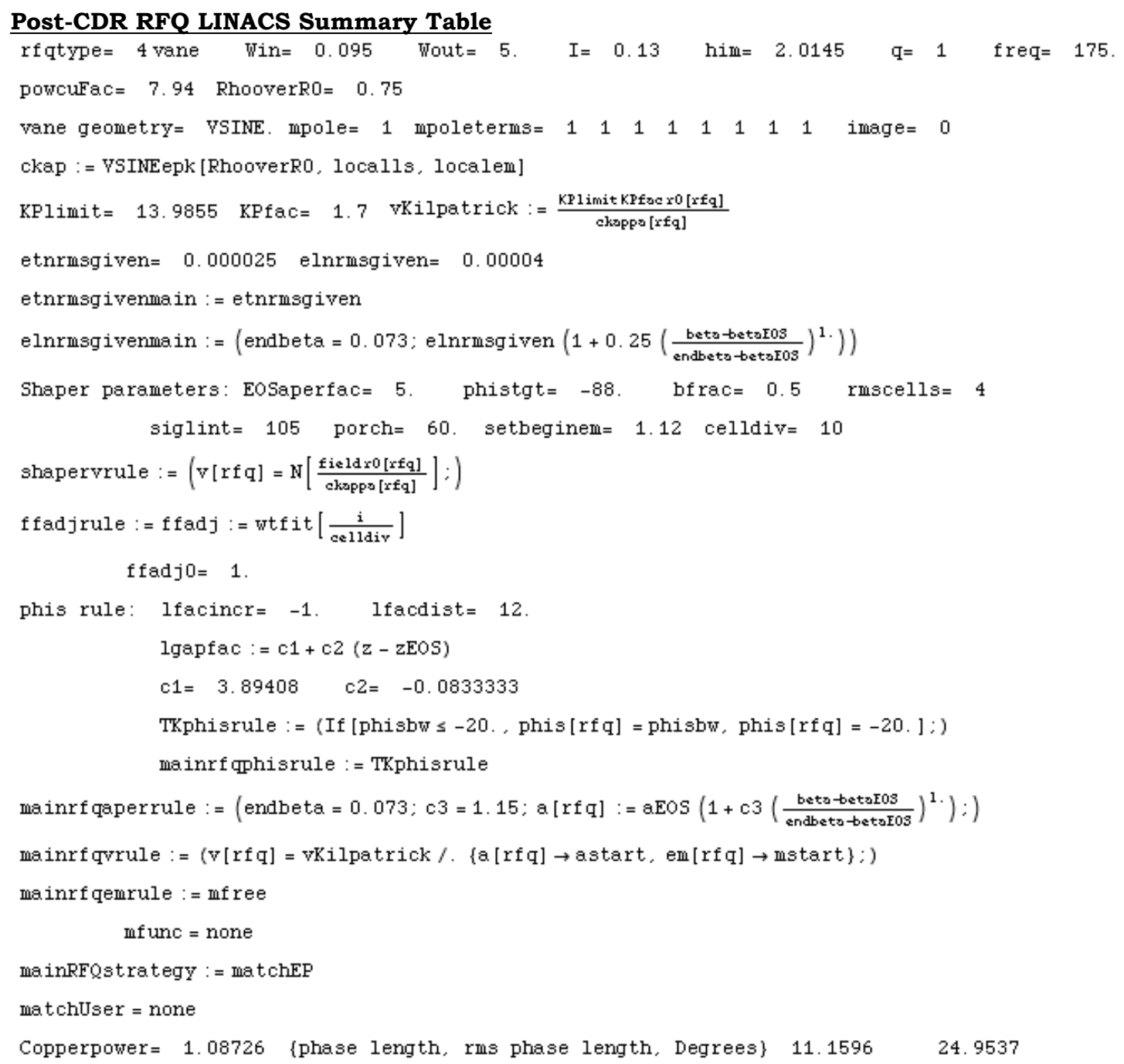

\section{Post-CDR RFQ PteqHI tapeinput}

\begin{tabular}{lllllllllllllllllll}
\hline run 1 & 0 & 0 & 0 & 2 & 0 & 0 & 0 & 0 & 0 & 0 & 0 & 0 & 0 & 0 & 0
\end{tabular}

title

IFMIF C27og 3

linac 10.095175 .2 .01451

tank $15 . \quad \begin{array}{llllllllllllllll}-90 & 0.1 & 0 & 1.0 & 0 & 1.0 & 0 & 0 & 1.0 & 10 & 1 & 36 & 0.0 & 0.0\end{array}$

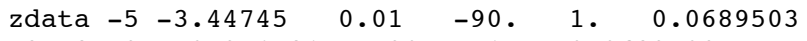

$\begin{array}{llllll}-2.58559 & 0.974581 & -90 & 1 & 1 . & 0.0689503\end{array}$

$\begin{array}{lllll}-1.72372 & 1.93916 & -90 . & 1 . & 0.0689503\end{array}$

$\begin{array}{lllllll}-0.861862 & 2.90374 & -90 & 1 . & 0.0689503 & 4\end{array}$

zdata -5

0 . $\quad 3.86832 \quad-90 . \quad 1.0 .0689503$

$\begin{array}{lllll}8.61862 & 4.62525 & -90 . & 1.00814 & 0.0689503\end{array}$

$\begin{array}{lllll}17.2372 & 5.38986 & -90 . & 1.02966 & 0.0689503\end{array}$

$\begin{array}{lllll}25.8559 & 6.15447 & -90 . & 1.06247 & 0.0689503\end{array}$

$\begin{array}{lllll}27.5796 & 6.3074 & -89.8473 & 1.07032 & 0.0689503\end{array}$

$\begin{array}{lllll}29.3034 & 6.46033 & -89.6496 & 1.07861 & 0.0689503\end{array}$

$\begin{array}{lllll}31.0275 & 6.61328 & -89.4519 & 1.08735 & 0.0689503\end{array}$

$\begin{array}{lllll}32.752 & 6.76627 & -89.2542 & 1.09653 & 0.0689503\end{array}$ 


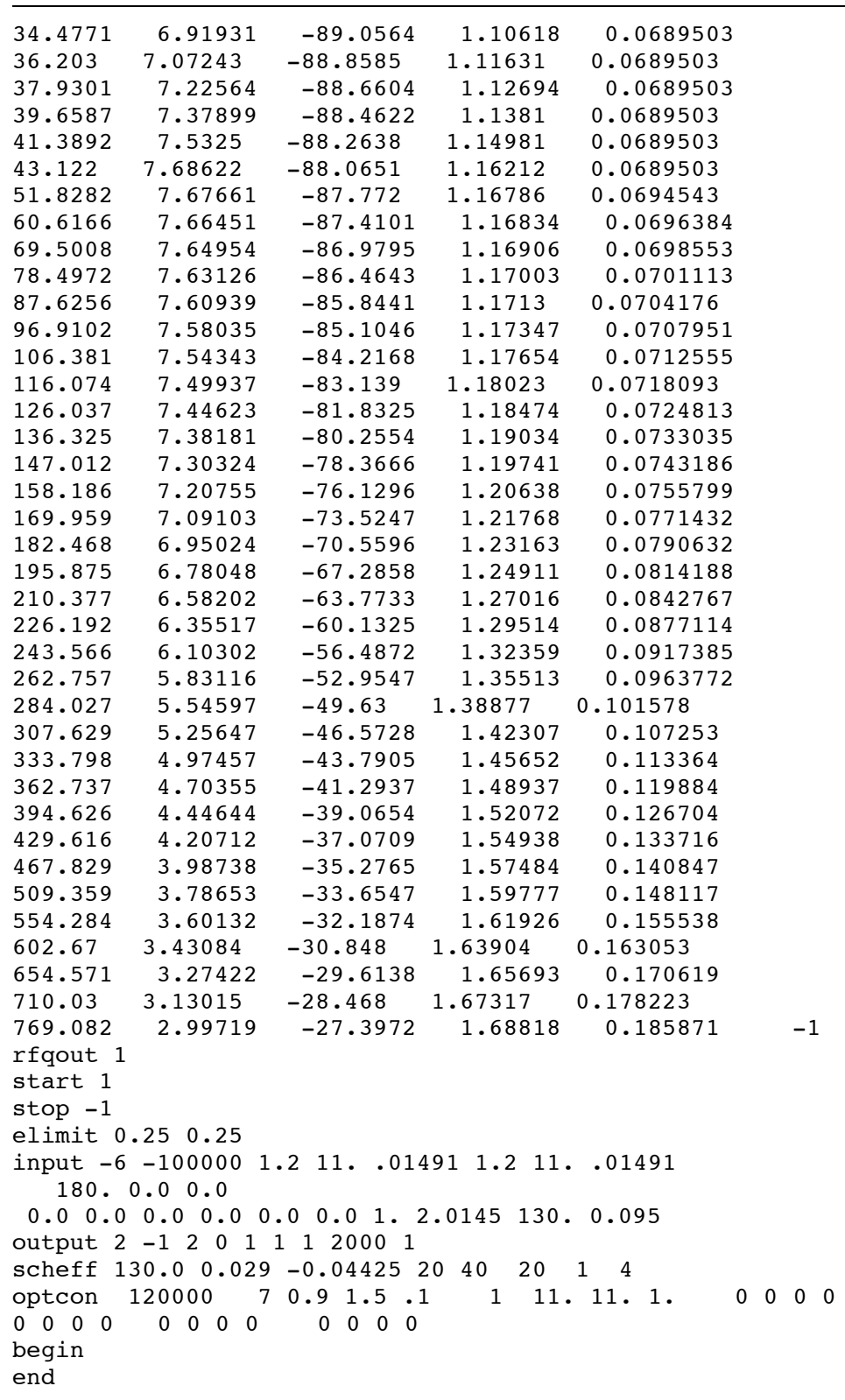

\section{Post-CDR RFQ Cell Table}

$\begin{array}{rlllllllllll}\text { nc } & \text { V } & \text { ws } & \text { beta } & \text { ez } & \text { capa } & \text { phi } & \text { a } & \text { m } & \text { b } & c l & t l \\ 0 & 0.069 & 0.0950 & 0.0101 & 0.000 & 0.00000 & -90.0 & 10.384 & 1.000 & 0.010 & 0.000 & 0.00 \\ 1 & 0.069 & 0.0950 & 0.0101 & 0.000 & 0.00000 & -90.0 & 1.052 & 1.000 & 0.975 & 0.862 & 0.86 \\ 2 & 0.069 & 0.0950 & 0.0101 & 0.000 & 0.00000 & -90.0 & 0.746 & 1.000 & 1.939 & 0.862 & 1.72 \\ 3 & 0.069 & 0.0950 & 0.0101 & 0.000 & 0.00000 & -90.0 & 0.609 & 1.000 & 2.904 & 0.862 & 2.59 \\ 4 & 0.069 & 0.0950 & 0.0101 & 0.000 & 0.00000 & -90.0 & 0.528 & 1.000 & 3.868 & 0.862 & 3.45 \\ 5 & 0.069 & 0.0950 & 0.0101 & 0.000 & 0.00003 & -90.0 & 0.523 & 1.000 & 3.937 & 0.862 & 4.31 \\ 6 & 0.069 & 0.0950 & 0.0101 & 0.001 & 0.00013 & -90.0 & 0.518 & 1.000 & 4.014 & 0.862 & 5.17 \\ 7 & 0.069 & 0.0950 & 0.0101 & 0.002 & 0.00030 & -90.0 & 0.513 & 1.001 & 4.090 & 0.862 & 6.03 \\ 8 & 0.069 & 0.0950 & 0.0101 & 0.004 & 0.00053 & -90.0 & 0.508 & 1.001 & 4.167 & 0.862 & 6.89 \\ 9 & 0.069 & 0.0950 & 0.0101 & 0.007 & 0.00083 & -90.0 & 0.504 & 1.002 & 4.243 & 0.862 & 7.76 \\ 10 & 0.069 & 0.0950 & 0.0101 & 0.010 & 0.00119 & -90.0 & 0.499 & 1.003 & 4.319 & 0.862 & 8.62 \\ 11 & 0.069 & 0.0950 & 0.0101 & 0.013 & 0.00162 & -90.0 & 0.494 & 1.004 & 4.396 & 0.862 & 9.48 \\ 12 & 0.069 & 0.0950 & 0.0101 & 0.017 & 0.00211 & -90.0 & 0.490 & 1.005 & 4.472 & 0.862 & 10.34 \\ 13 & 0.069 & 0.0950 & 0.0101 & 0.021 & 0.00267 & -90.0 & 0.485 & 1.007 & 4.549 & 0.862 & 11.20 \\ 14 & 0.069 & 0.0950 & 0.0101 & 0.026 & 0.00330 & -90.0 & 0.481 & 1.008 & 4.625 & 0.862 & 12.07\end{array}$




\begin{tabular}{|c|c|c|c|c|c|c|c|c|c|c|c|}
\hline 15 & 59 & 950 & 01 & 032 & 00399 & -90.0 & 0.477 & 1.010 & .702 & 0.862 & .9 \\
\hline 16 & .069 & 0950 & 0101 & 0.038 & 0.00475 & -90.0 & 472 & 1 & 778 & & \\
\hline 17 & .069 & 50 & 101 & 0.045 & 57 & -90.0 & & & & & \\
\hline 18 & 0.069 & & & & 6 & $-9 c$ & & & & & \\
\hline 19 & 069 & 0950 & 0101 & 0.059 & 0.00742 & -90 & 460 & 7 & 08 & & 8 \\
\hline 20 & .069 & 950 & 0.0101 & 0.067 & 0.00844 & -90.0 & 456 & 1.020 & & & \\
\hline 21 & .069 & 50 & 0.0101 & 0.076 & 0.00952 & -90.0 & 452 & 1.022 & & & 10 \\
\hline 22 & .069 & 50 & 0.0101 & 0.085 & 0.01068 & -90.0 & 448 & 1.024 & .237 & & .96 \\
\hline 23 & 0.069 & 0950 & 0.0101 & 0.095 & 90 & -90.0 & & 1.027 & 13 & & 9.82 \\
\hline 24 & & & & & & & & & & & \\
\hline 25 & .069 & & 1 & & & -90.0 & & 1.032 & & & \\
\hline 26 & .069 & 0. & 0.0101 & 0.128 & 0.0 & -90.0 & & & & & 2.41 \\
\hline 27 & .069 & & & & & & & & & & \\
\hline 28 & & & & & & & & & & & \\
\hline 29 & & & & & & & & & & & .99 \\
\hline 30 & .069 & 0.0950 & 0.0101 & 0.178 & 0.0 & & & & & & 25.86 \\
\hline 31 & .0 & & & & & & & & & & \\
\hline 32 & & & & & & & & & & & \\
\hline 33 & & & & & & & & & & & \\
\hline 34 & .069 & 50 & 0.01 & 0.237 & 0.0 & -9 & & & & & .30 \\
\hline 35 & .069 & & & & & -89.9 & & & & & \\
\hline 36 & & & & & & & & & & & \\
\hline 37 & 9 & & & & & & & & & & 89 \\
\hline 38 & 069 & & & & & & & & & & \\
\hline 39 & .0 & & & & & & & & & & \\
\hline 40 & 060 & & & 0.342 & & -89.5 & & & & & \\
\hline 41 & 0.069 & 51 & 1 & 0.361 & & -89.4 & & 1. & & & .34 \\
\hline 42 & .069 & & & & & & & & & & \\
\hline 43 & & & & & & & & & & & \\
\hline 44 & & & & & & & & & & & \\
\hline 45 & 0069 & & 0 & 0.444 & & -89.0 & 72 & & & & .79 \\
\hline 46 & & & & & & & & & & & \\
\hline 47 & & & & & & & & & & & \\
\hline 48 & & & & & & & & & & & \\
\hline 49 & 0 & & & & & & & & & & 24 \\
\hline 50 & .0 & & & & & & & & & & \\
\hline 51 & & & & & & & & & & & \\
\hline 52 & 0 & & & 0 & & -88.3 & & & & & 84 \\
\hline 53 & 0 & & & & & & & & & & \\
\hline 54 & & & & & & & & & & & \\
\hline 55 & 0 & & & & & -8 & & & & & 44 \\
\hline 56 & 0 & 4 & 0.0 & 0.687 & & -88.0 & & 1. & & & .31 \\
\hline 57 & & & & & & & & & & & \\
\hline 58 & & & & & & & & & & & \\
\hline 59 & & & & & & -87 & & & & & .92 \\
\hline 60 & 0.069 & & 0.01 & 0.687 & & -87.9 & & 1.1 & & & .79 \\
\hline 61 & & & & & & & & & & & \\
\hline 62 & & & & & & & & & & & \\
\hline 63 & & & & & & -8 & & & & & 41 \\
\hline 64 & & & & & & & & & & & \\
\hline 65 & & & & & & & & & & & \\
\hline 66 & & & & & & -8 & & & & & 3 \\
\hline 67 & 0.0 & & & 0.6 & & -87.7 & & 1. & & & 7.91 \\
\hline 68 & & & & & & & & & & & \\
\hline 69 & & & & & & & & & & & \\
\hline 70 & & & & & & -8 & & & & & 55 \\
\hline 71 & 0.070 & & 0.0 & 0.689 & & -87.5 & & 1. & & & 1.43 \\
\hline 72 & ( & & & & & & & & & & .31 \\
\hline 73 & & & & & & & & & & & \\
\hline 74 & & & & & & & & & & & \\
\hline 75 & 0.070 & 0 & 0.0 & 0.690 & 0.0 & -87.4 & & 1.1 & & & 4.96 \\
\hline 76 & & & & & & & & & & & .85 \\
\hline 77 & & & & & & & & & & & \\
\hline 78 & & & & & & & & & & & \\
\hline 79 & .0 & & & 0.6 & & -87 & & & & & .51 \\
\hline 80 & 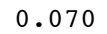 & & & & & & & & & & \\
\hline 81 & & & & & & & & & & & \\
\hline 82 & .0 & & & 0 . & & & & & & & .18 \\
\hline 83 & 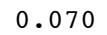 & & & & & & & & & & 72.0 \\
\hline 84 & & & & & & & & & & & \\
\hline 85 & .070 & & & 0.693 & & -86.9 & 0.349 & 1 & 7. & $0 . \varepsilon$ & 73.86 \\
\hline
\end{tabular}




\begin{tabular}{|c|c|c|c|c|c|c|c|c|c|c|c|}
\hline 86 & & 27 & 05 & 93 & 89 & -86.9 & 349 & 9 & 646 & 6 & 4.7 \\
\hline 87 & & & & & & & & & & & \\
\hline 88 & .070 & 32 & & & & -86.8 & & & & & \\
\hline 89 & & 1035 & & 0.695 & & -86.7 & & & & & \\
\hline 90 & & & & & & & & & & & \\
\hline 91 & .070 & & & & & & & & & & \\
\hline 92 & .070 & 44 & & & 971 & & & & & & \\
\hline 93 & & & & & & & & & & & 81.06 \\
\hline 94 & & & & & & & & & & & \\
\hline 95 & & & & & & & & & & & \\
\hline 96 & & & & & & & & & & & \\
\hline 97 & & & & & & & & & & & \\
\hline 98 & & & & & & & & & & & \\
\hline 99 & & & & & & & & & & & \\
\hline 100 & 0 & & & & & & & & & & \\
\hline 101 & & & & & & & & & & & \\
\hline & & & & & & & & & & & \\
\hline 103 & & & & & & & & & & & \\
\hline 104 & 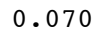 & & & & & & & & & & \\
\hline 105 & & & & & & & & & & & \\
\hline 5 & & & & & & & & & & & \\
\hline 107 & & & & & & & & & & & \\
\hline 108 & & & & & & & & & & & \\
\hline 109 & & & & & & & & & & & \\
\hline & & & & & & & & & & & \\
\hline 111 & & & & & & & & & & & \\
\hline 112 & & & & & & & & & & & \\
\hline 11 & & & & & & & & & & & \\
\hline 11 & & & & & & & & & & & \\
\hline 115 & 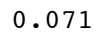 & & & & & & & & & & \\
\hline 116 & & & & & & & & & & & \\
\hline & & & & & & & & & & & \\
\hline & & & & & & & & & & & \\
\hline & & & & & & & & & & & \\
\hline & & & & & & & & & & & \\
\hline & & & & & & & & & & & \\
\hline 122 & & & & & & & & & & & \\
\hline & & & & & & & & & & & \\
\hline & & & & & & & & & & & \\
\hline & & & & & & & & & & & \\
\hline 126 & & & & & & & & & & & \\
\hline & & & & & & & & & & & \\
\hline 1 & & & & & & & & & & & \\
\hline 1 & & & & & & & & & & & \\
\hline 130 & & & & & & & & & & & \\
\hline & & & & & & & & & & & \\
\hline & & & & & & & & & & & \\
\hline & & & & & & & & & & & \\
\hline & & & & & & & & & & & \\
\hline & & & & & & & & & & & \\
\hline & & & & & & & & & & & \\
\hline 137 & & & & & & & & & & & .55 \\
\hline 1 & & & & & & & & & & & \\
\hline & & & & & & & & & & & \\
\hline & & & & & & & & & & & \\
\hline 141 & 0 & & & & & & & & & & \\
\hline & & & & & & & & & & & \\
\hline & & & & & & & & & & & \\
\hline 14 & & & & & & & & & & & \\
\hline 145 & & & & & & & & & & & 58 \\
\hline 14 & & & & & & & & & & & \\
\hline & & & & & & & & & & & \\
\hline 14 & & & & & & & & & & & \\
\hline 149 & & & & & & & & & & & \\
\hline & & & & & & & & & & & \\
\hline & & & & & & & & & & & \\
\hline 152 & & & & & & & & & & & \\
\hline 153 & 0 & & & 0 . & & & & & & & .83 \\
\hline & & & & & & & & & & & \\
\hline & & & & & & & & & & & \\
\hline 156 & 0.073 & 0.1426 & 0.0123 & 0.769 & 0.11043 & -79.9 & 0.360 & 1.192 & 7.367 & 1.056 & 141 \\
\hline
\end{tabular}




\begin{tabular}{|c|c|c|c|c|c|c|c|c|c|c|c|}
\hline & & & 124 & 771 & 102 & 9.7 & 361 & 1.192 & 360 & 1.060 & 143.04 \\
\hline 158 & .074 & & & 73 & & -79.5 & & & & & \\
\hline 159 & .074 & 50 & 0125 & 0.775 & 1223 & -79.4 & 361 & 94 & 344 & .069 & 17 \\
\hline 160 & & & 125 & 777 & 285 & & & & & & \\
\hline 61 & & & & & & & & & & & \\
\hline 162 & .074 & & & 82 & & -78.8 & & & & & \\
\hline 163 & .074 & & .0127 & 0.784 & & -78.6 & 0.363 & & & .087 & \\
\hline 164 & 017 & & & & & & & & & & \\
\hline 165 & & & & & & & & & & & \\
\hline 166 & & & & & & -7 & & & & & \\
\hline 167 & 075 & & & & & & & & & & \\
\hline 168 & & & & & & & & & & & \\
\hline 169 & & & & & & & & & & & \\
\hline 170 & & & & & & -77.1 & & & & & \\
\hline 171 & & & & & & & & & & & \\
\hline 172 & & & & & & & & & & & \\
\hline 173 & & & & & & & & & & & \\
\hline 174 & & & & & & & & & & & \\
\hline 175 & & & & & & & & & & & \\
\hline 17 & & & & & & & & & & & \\
\hline 177 & & & & & & & & & & & \\
\hline 178 & 6 & & & & & & & & & & \\
\hline 179 & & & & & & & & & & & \\
\hline 18 & & & & & & & & & & & \\
\hline & & & & & & & & & & & \\
\hline 182 & & & & & & & & & & & \\
\hline 183 & & & & & & & & & & & \\
\hline 18 & & & & & & & & & & & \\
\hline 18 & & & & & & & & & & & \\
\hline 186 & & & & & & & & & & & \\
\hline 187 & & & & & & & & & & & \\
\hline 18 & & & & & & & & & & & \\
\hline 189 & & & & & & & & 1 & & & \\
\hline 10 & & & & & & & & & & & \\
\hline 19 & & & & & & & & & & & \\
\hline 19 & & & & & & & & & & & \\
\hline 193 & & & & 0.876 & & -70.9 & & & & & \\
\hline 194 & & & & & & & & & & & \\
\hline 19 & & & & & & & & & & & \\
\hline 19 & & & & & & & & & & & \\
\hline 197 & & & & & & & & & & & \\
\hline 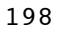 & & & & & & & & & & & \\
\hline 19 & & & & & & & & & & & \\
\hline 200 & & & & & & & & & & & \\
\hline & & & & & & & & & & & \\
\hline 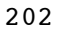 & & & & & & & & & & & \\
\hline 20 & & & & & & & & & & & \\
\hline 204 & & & & & & -6 & & 1 & & & \\
\hline & & & & & & & & & & & \\
\hline & & & & & & & & & & & \\
\hline 20 & & & & & & & & & & & \\
\hline 208 & & & & & & & & & & & \\
\hline 209 & & & & & & & & & & & \\
\hline & & & & & & & & & & & \\
\hline & & & & & & & & & & & \\
\hline 212 & & & & & & & & & & & \\
\hline & & & & & & & & & & & \\
\hline 21 & & & & & & & & & & & \\
\hline 215 & & & & & & & & & & & \\
\hline 216 & & & & & & & & & & & \\
\hline ? & & & & & & & & & & & \\
\hline 21 & & & & & & & & & & & \\
\hline 21 & & & & & & & & & & & \\
\hline 22 & & & & & & & & & & & \\
\hline & & & & & & & & & & & \\
\hline 22 & & & & & & & & & & & \\
\hline 223 & $0.0 \varepsilon$ & & & 1. & 32 & -60 & & 1. & & & .42 \\
\hline 224 & & & & 1 . & & & & & & & \\
\hline 2 & & & & & & & & & & & \\
\hline & & & & & & & & & & & \\
\hline 227 & .089 & .3714 & 0.0199 & 1.037 & 0.19885 & 0 & 0.408 & 1.303 & 6.282 & 1.704 & 2 \\
\hline
\end{tabular}




\begin{tabular}{|c|c|c|c|c|c|c|c|c|c|c|c|}
\hline & & & 201 & 42 & 091 & 8.7 & 10 & 6 & & 720 & \\
\hline 29 & 90 & 360 & 203 & 048 & 98 & -58.3 & & 309 & & 737 & \\
\hline 30 & .090 & 3936 & .0205 & 1.053 & 0.20508 & -57.9 & 0.412 & 1.312 & .207 & .754 & 40.35 \\
\hline 31 & & & & & & & & & & & \\
\hline 32 & 91 & & 209 & & & & & & & & \\
\hline 233 & .091 & .76 & 211 & 69 & 46 & -56.8 & & 21 & & 07 & \\
\hline 234 & .092 & .4260 & .0213 & 1.074 & 0.21362 & -56.5 & & 1.324 & & 825 & \\
\hline 235 & & & & & & & & & & & \\
\hline 36 & & & & & & & & & & & \\
\hline 237 & & & & & & -55.4 & & & & & \\
\hline 238 & 090 & & & & & -55 & & & & & \\
\hline 239 & & & & & & & & & & & \\
\hline 240 & 4 & & & & & -54.3 & & & & & \\
\hline 241 & .095 & & & & & & & & & & \\
\hline 242 & & & & & & & & & & & \\
\hline & & & & & & & & & & & \\
\hline 244 & & & & & & -53.0 & & & & & \\
\hline 245 & 007 & & & & & & & & & & \\
\hline 246 & & & & & & & & & & & \\
\hline & & & & & & & & & & & \\
\hline 248 & & & & & & -51.6 & & & & & \\
\hline 249 & & & & & & & & & & & \\
\hline 250 & & & & & & & & & & & \\
\hline 25 & & & & & & & & & & & \\
\hline 252 & & & & & & -50.3 & & & & & \\
\hline 253 & & & & & & & & & & & \\
\hline 254 & & & & & & -4 & & & & & \\
\hline 25 & & & & & & & & & & & \\
\hline 256 & & & & & & & & & & & \\
\hline 257 & & & & & & & & & & & \\
\hline $25 \xi$ & & & & & & & & & & & \\
\hline 25 & & & & & & & & & & & \\
\hline 260 & & & & & & & & & & & \\
\hline 261 & & & & & & & & & & & \\
\hline 262 & & & & & & & & & & & \\
\hline 263 & & & & & & & & & & & \\
\hline 264 & & & & & & & & & & & \\
\hline 265 & & & & & & & & & & & \\
\hline 266 & & & & & & & & & & & \\
\hline 267 & & & & & & & & & & & \\
\hline 268 & & & & & & & & & & & \\
\hline 26 & & & & & & & & & & & \\
\hline 27 & & & & & & & & & & & \\
\hline 271 & & & & & & & & & & & \\
\hline 21 & & & & & & & & & & & \\
\hline 27 & & & & & & & & & & & \\
\hline 27 & & & & & & & & & & & \\
\hline 275 & & & & & & & & & & & \\
\hline & & & & & & & & & & & \\
\hline 21 & & & & & & & & & & & \\
\hline 278 & & & & & & & & & & & \\
\hline 279 & & & & & & & & & & & \\
\hline 280 & & & & & & & & & & & \\
\hline 28 & & & & & & & & & & & \\
\hline 28 & & & & & & -41 & & & & & \\
\hline 28 & & & & & & & & & & & \\
\hline & & & & & & & & & & & \\
\hline 28 & & & & & & & & & & & \\
\hline 286 & 0 & & & & & 8 & & & & & \\
\hline 28 & & & & & & & & & & & \\
\hline ? & & & & & & & & & & & \\
\hline 28 & & & & & & & & & & & \\
\hline 290 & 0 & & & & & & & & & & \\
\hline 29 & & & & & & & & & & & \\
\hline & & & & & & & & & & & \\
\hline 29 & & & & & & & & & & & \\
\hline 294 & 0 & & & 6 & & & & & & & \\
\hline 29 & & & & & & & & & & & \\
\hline 2 & & & & & & & & & & & \\
\hline 29 & & & & & & & & & & & \\
\hline 98 & .129 & .5403 & 0.0405 & 1.313 & 60 & -3 & 39 & 1. & 4 . & 68 & 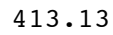 \\
\hline
\end{tabular}




\begin{tabular}{|c|c|c|c|c|c|c|c|c|c|c|c|}
\hline & & 686 & 409 & 314 & 331 & 38.0 & 541 & כנכיו & 24 & 500 & . \\
\hline 300 & 31 & 72 & & & & -37.8 & & & & & \\
\hline 301 & 132 & .6262 & 0416 & 1.317 & 0.35666 & -37.6 & 0.546 & 1.541 & .277 & .564 & 23.72 \\
\hline 02 & & 556 & & & 830 & & & & & & \\
\hline 03 & & & & & & & & & & & \\
\hline 304 & & & & & & -37.1 & & & & & \\
\hline 305 & 134 & .7458 & & 1.322 & & -36.9 & & & & .692 & \\
\hline 306 & & & & & & & & & & & \\
\hline 307 & & & & & & & & & & & \\
\hline 308 & & & & & & & & & & & \\
\hline 309 & 137 & & & & & & & & & & \\
\hline 310 & & & & & & & & & & & \\
\hline 311 & & & & & & & & & & & \\
\hline 312 & & & & & 0. & & & & & & \\
\hline 313 & & & & & & & & & & & \\
\hline 314 & & & & & & & & & & & \\
\hline 315 & & & & & & & & & & & \\
\hline 316 & & & & & & & & & & & \\
\hline 317 & & & & & & & & & & & \\
\hline & & & & & & & & & & & \\
\hline 319 & & & & & & -34.4 & & & & & \\
\hline 320 & .145 & & & & & & & & & & \\
\hline 321 & & & & & & & & & & & \\
\hline 32 & & & & & & & & & & & \\
\hline 323 & & & & & & & & & & & \\
\hline 324 & & & & & & & & & & & \\
\hline 325 & & & & & & & & & & & \\
\hline 32 & & & & & & & & & & & \\
\hline 327 & & & & & & & & & & & \\
\hline 328 & & & & & & & & & & & \\
\hline 32 & & & & & & & & & & & \\
\hline 33 & & & & & & & & & & & \\
\hline 331 & & & & & & & & & & & \\
\hline & & & & & & & & & & & \\
\hline 33 & & & & & & & & & & & \\
\hline 334 & & & & & & & & & & & \\
\hline 335 & & & & & & & & & & & \\
\hline 36 & & & & & & & & & & & \\
\hline 33 & & & & & & & & & & & \\
\hline 338 & & & & & & & & & & & \\
\hline 3 & & & & & & & & & & & \\
\hline 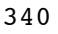 & & & & & & & & & & & \\
\hline 34 & & & & & & & & & & & \\
\hline 342 & & & & & & & & & & & \\
\hline & & & & & & & & & & & \\
\hline \pm & & & & & & & & & & & \\
\hline 34 & & & & & & & & & & & \\
\hline 346 & & & & & & & & & & & \\
\hline & & & & & & & & & & & \\
\hline & & & & & & & & & & & \\
\hline 349 & & & & & & & & & & & \\
\hline 350 & 1 & & & 1.342 & & & & & & & \\
\hline 35 & & & & & & & & & & & \\
\hline 35 & & & & & & & & & & & \\
\hline 35 & & & & & & & & & & & \\
\hline 354 & & & & & & & & & & & \\
\hline & & & & & & & & & & & \\
\hline 35 & & & & & & & & & & & \\
\hline 357 & & & & & & & & & & & \\
\hline 35 & & & & & & & & & & & \\
\hline & & & & & & & & & & & \\
\hline 36 & & & & & & & & & & & \\
\hline 361 & & & & & & & & & & & \\
\hline 36 & & & & & & & & & & & \\
\hline 3 & & & & & & & & & & & \\
\hline 36 & & & & & & & & & & & \\
\hline 365 & 0.1 & & & 1.341 & 0 . & -28.4 & & & & & 21.92 \\
\hline 366 & & & & & & & & & & & \\
\hline ? & & & & & & & & & & & \\
\hline & & & & & & & & & & & \\
\hline 69 & .182 & 781 & .0690 & 1.340 & 0.43482 & 9 & 0.719 & 1.681 & 3.062 & 5. & 71 \\
\hline
\end{tabular}




\begin{tabular}{lllllllllllll}
\hline 370 & 0.183 & 4.5332 & 0.0694 & 1.340 & 0.43560 & -27.8 & 0.722 & 1.682 & 3.049 & 5.943 & 751.27 \\
371 & 0.184 & 4.5887 & 0.0698 & 1.340 & 0.43638 & -27.7 & 0.725 & 1.684 & 3.036 & 5.979 & 757.25 \\
372 & 0.184 & 4.6445 & 0.0702 & 1.340 & 0.43714 & -27.6 & 0.727 & 1.685 & 3.023 & 6.015 & 763.27 \\
373 & 0.185 & 4.7008 & 0.0706 & 1.339 & 0.43790 & -27.5 & 0.730 & 1.687 & 3.010 & 6.052 & 769.32 \\
374 & 0.186 & 4.7574 & 0.0711 & 1.339 & 0.43865 & -27.4 & 0.733 & 1.688 & 2.997 & 6.088 & 775.41 \\
375 & 0.187 & 4.8145 & 0.0715 & 1.339 & 0.43939 & -27.3 & 0.735 & 1.690 & 2.984 & 6.124 & 781.53 \\
376 & 0.187 & 4.8719 & 0.0719 & 1.339 & 0.44013 & -27.2 & 0.738 & 1.691 & 2.972 & 6.160 & 787.69 \\
377 & 0.188 & 4.9297 & 0.0723 & 1.339 & 0.44086 & -27.1 & 0.741 & 1.692 & 2.959 & 6.197 & 793.89 \\
378 & 0.189 & 4.9878 & 0.0728 & 1.339 & 0.44158 & -27.0 & 0.743 & 1.694 & 2.947 & 6.233 & 800.12
\end{tabular}

\section{CDR RFQ PteqHI tapeinput}

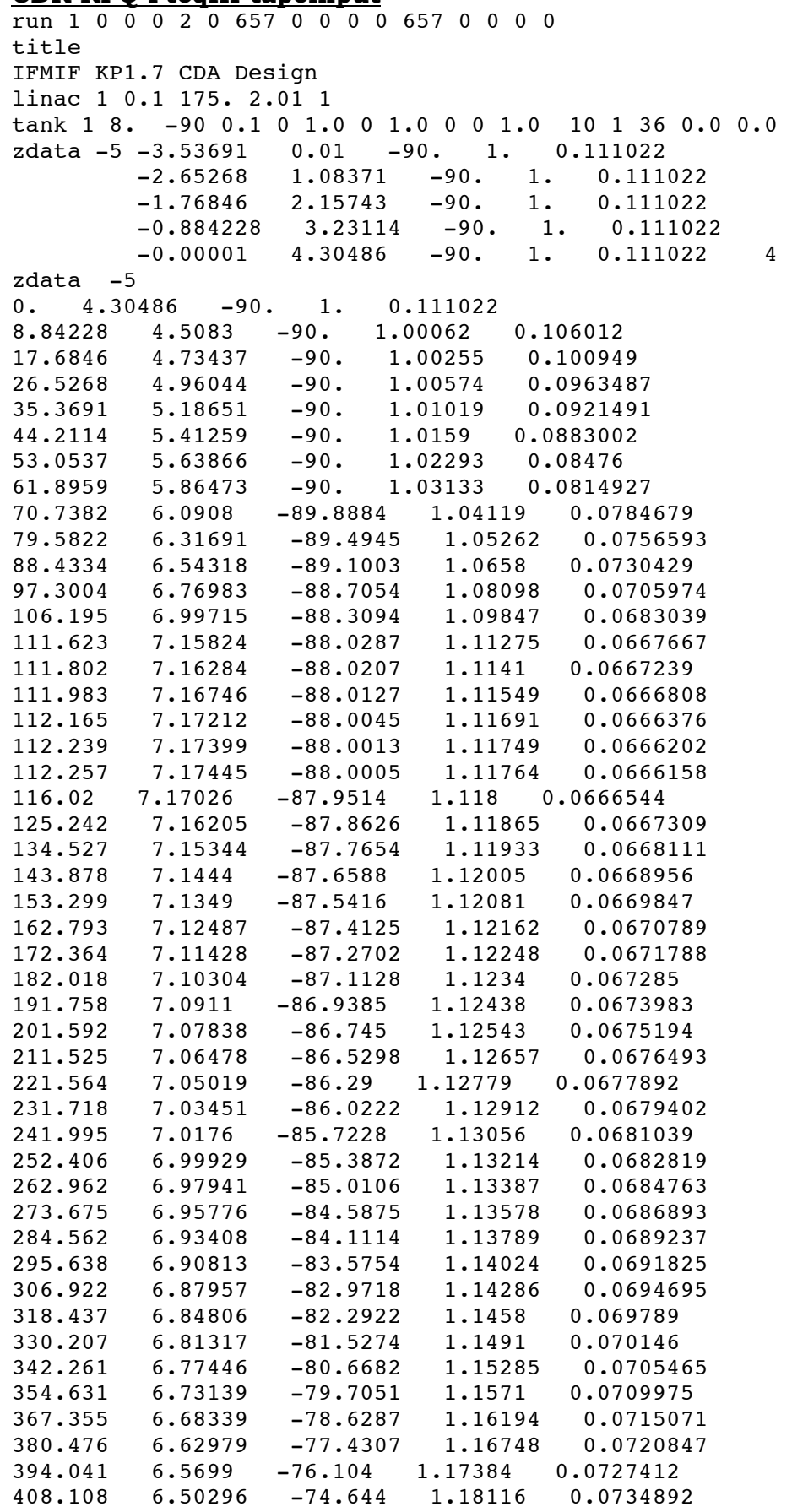




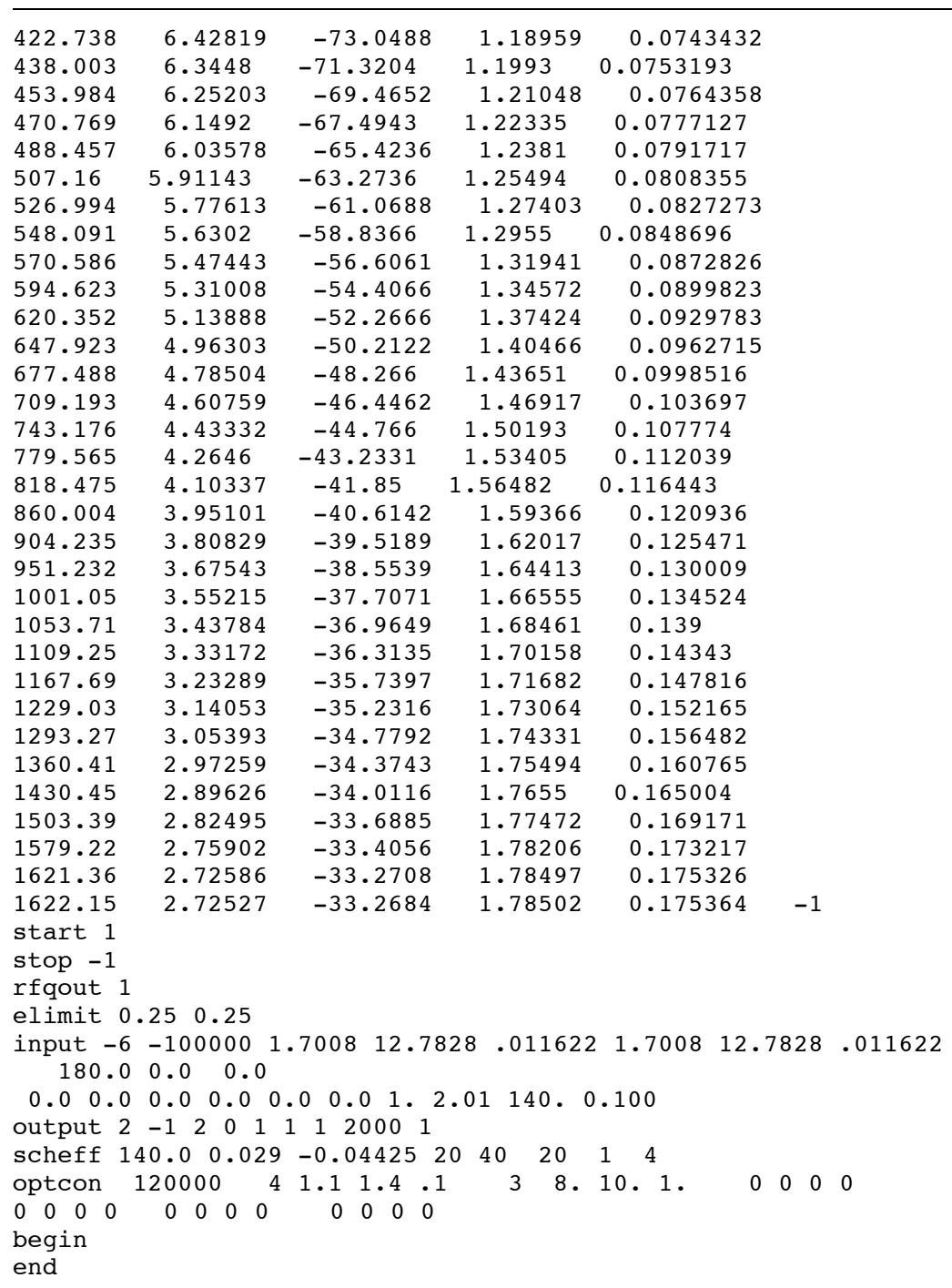

\section{CDR RFQ Cell Table}

$\begin{array}{rlllllllllll}\text { nc } & \mathrm{V} & \text { ws } & \text { beta } & \text { ez } & \text { Capa } & \text { phi } & \text { a } & \text { m } & \text { b } & c l & t l \\ 0 & 0.111 & 0.1000 & 0.0103 & 0.000 & 0.00000 & -90.0 & 13.192 & 1.000 & 0.010 & 0.000 & 0.00 \\ 1 & 0.111 & 0.1000 & 0.0103 & 0.000 & 0.00000 & -90.0 & 1.266 & 1.000 & 1.085 & 0.885 & 0.89 \\ 2 & 0.111 & 0.1000 & 0.0103 & 0.000 & 0.00000 & -90.0 & 0.898 & 1.000 & 2.160 & 0.885 & 1.77 \\ 3 & 0.111 & 0.1000 & 0.0103 & 0.000 & 0.00000 & -90.0 & 0.733 & 1.000 & 3.235 & 0.885 & 2.66 \\ 4 & 0.111 & 0.1000 & 0.0103 & 0.000 & 0.00000 & -90.0 & 0.635 & 1.000 & 4.310 & 0.885 & 3.54 \\ 5 & 0.111 & 0.1000 & 0.0103 & 0.000 & 0.00000 & -90.0 & 0.633 & 1.000 & 4.325 & 0.885 & 4.43 \\ 6 & 0.110 & 0.1000 & 0.0103 & 0.000 & 0.00000 & -90.0 & 0.630 & 1.000 & 4.346 & 0.885 & 5.31 \\ 7 & 0.110 & 0.1000 & 0.0103 & 0.000 & 0.00000 & -90.0 & 0.627 & 1.000 & 4.366 & 0.885 & 6.20 \\ 8 & 0.109 & 0.1000 & 0.0103 & 0.000 & 0.00000 & -90.0 & 0.624 & 1.000 & 4.386 & 0.885 & 7.08 \\ 9 & 0.109 & 0.1000 & 0.0103 & 0.000 & 0.00000 & -90.0 & 0.621 & 1.000 & 4.407 & 0.885 & 7.97 \\ 10 & 0.108 & 0.1000 & 0.0103 & 0.000 & 0.00000 & -90.0 & 0.618 & 1.000 & 4.427 & 0.885 & 8.85 \\ 11 & 0.108 & 0.1000 & 0.0103 & 0.000 & 0.00000 & -90.0 & 0.615 & 1.000 & 4.448 & 0.885 & 9.74 \\ 12 & 0.107 & 0.1000 & 0.0103 & 0.000 & 0.00000 & -90.0 & 0.613 & 1.000 & 4.468 & 0.885 & 10.62 \\ 13 & 0.107 & 0.1000 & 0.0103 & 0.003 & 0.00029 & -90.0 & 0.610 & 1.001 & 4.488 & 0.885 & 11.51 \\ 14 & 0.106 & 0.1000 & 0.0103 & 0.003 & 0.00029 & -90.0 & 0.607 & 1.001 & 4.509 & 0.885 & 12.39 \\ 15 & 0.105 & 0.1000 & 0.0103 & 0.003 & 0.00029 & -90.0 & 0.604 & 1.001 & 4.531 & 0.885 & 13.28 \\ 16 & 0.105 & 0.1000 & 0.0103 & 0.004 & 0.00030 & -90.0 & 0.601 & 1.001 & 4.554 & 0.885 & 14.16 \\ 17 & 0.104 & 0.1000 & 0.0103 & 0.004 & 0.00030 & -90.0 & 0.598 & 1.001 & 4.577 & 0.885 & 15.05 \\ 18 & 0.104 & 0.1000 & 0.0103 & 0.004 & 0.00030 & -90.0 & 0.595 & 1.001 & 4.599 & 0.885 & 15.93 \\ 19 & 0.103 & 0.1000 & 0.0103 & 0.007 & 0.00061 & -90.0 & 0.592 & 1.002 & 4.622 & 0.885 & 16.82 \\ 20 & 0.103 & 0.1000 & 0.0103 & 0.007 & 0.00062 & -90.0 & 0.589 & 1.002 & 4.644 & 0.885 & 17.70\end{array}$




\begin{tabular}{|c|c|c|c|c|c|c|c|c|c|c|c|}
\hline 21 & 02 & 000 & 103 & 007 & 0062 & -90.0 & 586 & 1.002 & 4.667 & 885 & 18.59 \\
\hline 22 & 02 & 000 & 0103 & 007 & 0.00063 & 90.0 & 583 & 1.002 & & & \\
\hline 23 & 101 & 00 & 0103 & 0.007 & 0.00063 & -90.0 & 80 & 1.002 & & & \\
\hline 24 & 101 & .1000 & 0.0103 & 0.011 & 0.00096 & -90.0 & 0.577 & 1.003 & 4.735 & & 1.25 \\
\hline 25 & & 000 & 103 & & 0096 & & & & & & \\
\hline 26 & 100 & & 0103 & & & -90 & & 1.003 & & & \\
\hline 27 & .100 & 00 & 0103 & & 31 & -90.0 & & 1.004 & & & \\
\hline 28 & 099 & .1000 & 0.0103 & 0.015 & 0.00132 & -90.0 & & 1.004 & & & \\
\hline 29 & 099 & 0 & & & & & & & & & \\
\hline 30 & & & & & & & & & & & \\
\hline 31 & .098 & & 0103 & & & -90.0 & & 05 & & & \\
\hline 32 & 097 & & & & & & & & & & \\
\hline 33 & 7 & & & & & & & & & & \\
\hline 34 & & & & & & & & & & & \\
\hline 35 & 6 & & 3 & & & -90.0 & & & & & \\
\hline 36 & 095 & & & & & & & & & & \\
\hline 37 & 0 & & & & & & & & & & \\
\hline 38 & & & & & & -90.0 & & & & & \\
\hline 39 & 0 & & & & & & & & & & \\
\hline 40 & 0 & & & & & & & & & & \\
\hline 41 & 0 & & & & & & & & & & \\
\hline 42 & & & & & & & & & & & \\
\hline 43 & 0 & & & & & & & & & & \\
\hline 44 & 0 & & & & & & & & & & \\
\hline 45 & 0 & & & & & & & & & & \\
\hline 46 & & & & & & & & & & & \\
\hline 47 & .09 & & & & & & & & & & \\
\hline 48 & & & & & & & & & & & \\
\hline 49 & & & & & & & & & & & \\
\hline 50 & $.0 s$ & & & & & -9 & 1 & & & & \\
\hline 51 & 08 & & & & & & & & & & \\
\hline 52 & 0 & & & & & & & & & & \\
\hline 53 & .0 & & & & & & & & & & \\
\hline 54 & 0 & & 0.0 & & & -90 & & 1. & & & \\
\hline 55 & 0 & & & & & & & & & & \\
\hline 56 & 0 & & & & & -9 & & & & & \\
\hline 57 & & & & & & & & & & & \\
\hline 58 & .087 & & & & & -90.0 & & 1 . & & & \\
\hline 59 & & & & & & & & & & & \\
\hline 60 & 0 & & & & & -9 & & & & & \\
\hline 61 & & & & & & & & & & & \\
\hline 62 & & & & & & & & & & & \\
\hline 63 & & & & & & & & & & & \\
\hline 64 & 0 & & & & & -9 & & & & & \\
\hline 65 & . & & 3 & & & -90.0 & 78 & 1. & & & 54 \\
\hline 66 & & & & & & & & & & & \\
\hline 67 & & & & & & & & & & & \\
\hline 68 & & & & & & -9 & & & & & \\
\hline 69 & 0.0 & 2 & 3 & & & $-9 c$ & & 1. & & & 08 \\
\hline 70 & & & & & & & & & & & \\
\hline 71 & & & & & & & & & & & \\
\hline 72 & $.0 \xi \quad-2$ & & & & & & & & & & \\
\hline 73 & 0.08 & 2 & & & & -90.0 & & & & & 62 \\
\hline 74 & & & & & & & & & & & \\
\hline 75 & & & & & & & & & & & \\
\hline 76 & 0 & & & & & & & & & & \\
\hline 77 & & & & & & & & & & & \\
\hline 78 & & & & & & & & & & & \\
\hline 79 & & & & & & & & & & & \\
\hline 80 & & & 3 & & 0.0 & -89.9 & & & & & 0.82 \\
\hline 81 & 0 & & & & & & & & & & 70 \\
\hline 82 & & & & & & & & & & & \\
\hline 83 & & & & & & & & & & & \\
\hline 84 & 0.0 & & 3 & & & -89 & & & & & 36 \\
\hline 85 & 0 & & & & & & & & & & \\
\hline 86 & & & & & & & & & & & \\
\hline 87 & & & & & & & & & & & \\
\hline 88 & 0.077 & 0.1003 & 0.0103 & 0.186 & 0.02127 & -89.7 & 0.433 & 1.046 & .184 & 0.8 & 90 \\
\hline 89 & (2) & & 4 & & 4 & & & & & & \\
\hline 30 & 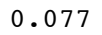 & & & & & & & & & & \\
\hline 91 & .076 & 03 & 0.0104 & 0.196 & 297 & -89.6 & 27 & 1.049 & 6.251 & 0.887 & 80.56 \\
\hline
\end{tabular}




\begin{tabular}{|c|c|c|c|c|c|c|c|c|c|c|c|}
\hline 92 & 76 & 04 & 104 & 00 & 2348 & 6 & 426 & 050 & 274 & 887 & \\
\hline 93 & 076 & & 0104 & 209 & 51 & & & .052 & & & \\
\hline 94 & .076 & & & & & & & 53 & & & \\
\hline 95 & 075 & & 0104 & 0.216 & & -89.5 & & 1.054 & & & \\
\hline 96 & & & & & & & & & & & \\
\hline 97 & .075 & & & & & & & & & & \\
\hline 98 & .075 & & & & & -89.3 & & & & & \\
\hline 99 & .074 & & & & & & & & & & \\
\hline 100 & . & & & & & & & & & & \\
\hline 101 & & & & & & & & & & & \\
\hline 102 & .074 & & & & & - & & & & & \\
\hline 103 & & & & & & & & & & & \\
\hline 104 & & & & & & & & & & & \\
\hline 105 & & & & & & & & & & & \\
\hline 106 & 3 & & & & & & & 69 & & & \\
\hline 107 & & & & & & & & & & & \\
\hline 108 & & & & & & & & & & & \\
\hline 109 & & & & & & & & & & & \\
\hline 110 & .0 & & & & & & & & & & \\
\hline 111 & 0 & & & & & & & & & & \\
\hline 112 & 0 & & & & & & & & & & \\
\hline & & & & & & & & & & & \\
\hline 114 & .0 & & & & & & & & & & \\
\hline 115 & & & & & & & & & & & \\
\hline 116 & & & & & & & & & & & \\
\hline & & & & & & & & & & & \\
\hline 118 & & & & & & & & & & & \\
\hline 119 & & & & & & & & & & & \\
\hline & & & & & & & & & & & \\
\hline 121 & & & & & & & & & & & \\
\hline 122 & & & & & & & & & & & \\
\hline 123 & & & & & & & & & & & \\
\hline 12 & & & & & & & & & & & \\
\hline 125 & & & & & & & & & & & \\
\hline 126 & & & & & & & & & & & \\
\hline 127 & & & & & & & & & & & \\
\hline 128 & & & & & & & & & & & \\
\hline 129 & & & & & & & & & & & \\
\hline & & & & & & & & & & & \\
\hline & & & & & & & & & & & \\
\hline & & & & & & & & & & & \\
\hline & & & & & & & & & & & \\
\hline & & & & & & & & & & & \\
\hline & & & & & & & & & & & \\
\hline 136 & & & & & & -87 & 51 & & & & \\
\hline & & & & & & & & & & & \\
\hline & & & & & & & & & & & \\
\hline & & & & & & & & & & & \\
\hline 140 & & & & & & -8 & & & & & \\
\hline & & & & & & & & & & & \\
\hline & & & & & & & & & & & \\
\hline & & & & & & & & & & & \\
\hline 144 & .06 & & & & & -8 & & & & & \\
\hline & & & & & & & & & & & \\
\hline & & & & & & & & & & & \\
\hline & & & & & & & & & & & \\
\hline 148 & & & & & & & & & & & \\
\hline & & & & & & & & & & & \\
\hline & & & & & & & & & & & \\
\hline & & & & & & -8 & & & & & \\
\hline 152 & 0 & & & & & & & & & & \\
\hline & & & & & & & & & & & \\
\hline & & & & & & & & & & & \\
\hline 155 & & & & & & & & & & & \\
\hline 1 & & & & & & & & & & & \\
\hline & & & & & & & & & & & \\
\hline & & & & & & & & & & & \\
\hline 159 & .06 & 3 & 0.01 & & 0 . & -87 & 0.361 & 1. & & & .47 \\
\hline 1 & 0 & & & & & & & & & & \\
\hline & & & & & & & & & & & \\
\hline 1 & .067 & & & & & -877 & 52 & 1 & & 9 & \\
\hline
\end{tabular}




\begin{tabular}{|c|c|c|c|c|c|c|c|c|c|c|c|}
\hline & 67 & 79 & 107 & 75 & 496 & 7 & 362 & 20 & 7 & 0 & \\
\hline 164 & .067 & & 07 & & 96 & -87.7 & & 120 & & & \\
\hline 165 & .067 & 082 & 0.0108 & 0.471 & 0.06503 & -87.7 & .362 & 1.120 & & .921 & \\
\hline 166 & 067 & 084 & 108 & & 509 & & & 120 & & & \\
\hline 67 & & & & & & & & & & & \\
\hline 168 & .067 & & & & & -87.6 & & 120 & & & \\
\hline 169 & .067 & & .0108 & 0.472 & 0.06522 & -87.6 & & 1.120 & & & \\
\hline 170 & 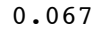 & & & & & & & & & & \\
\hline 171 & & & & & & & & & & & \\
\hline 172 & & & & & & & & & & & \\
\hline 173 & 067 & & & & & & & & & & \\
\hline 174 & & & & & & & & & & & \\
\hline 175 & & & & & & & & & & & \\
\hline 176 & .067 & & & & & & & & & & \\
\hline 177 & & & & & & & & & & & \\
\hline 178 & & & & & & & & & & & \\
\hline 179 & & & & & & & & & & & \\
\hline 180 & & & & & & & & & & & \\
\hline 181 & & & & & & & & & & & \\
\hline 18 & & & & & & & & & & & \\
\hline 18 & & & & & & & & & & & \\
\hline 184 & .067 & & & & & & & & & & \\
\hline 185 & & & & & & & & & & & \\
\hline 186 & & & & & & & & & & & \\
\hline & & & & & & & & & & & \\
\hline 188 & .067 & & & & & & & & & & \\
\hline 189 & & & & & & & & & & & \\
\hline 19 & 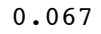 & & & & & & & & & & \\
\hline 19 & & & & & & & & & & & \\
\hline 192 & & & & & & & & & & & \\
\hline 193 & & & & & & & & & & & \\
\hline 19 & & & & & & & & & & & \\
\hline 19 & & & & & & & & & & & \\
\hline 19 & & & & & & & & & & & \\
\hline 19 & & & & & & & & & & & \\
\hline 19 & & & & & & & & & & & \\
\hline 199 & 0 & & & & & -8 & & 1.123 & & & \\
\hline م0 & & & & & & & & & & & \\
\hline 2 & & & & & & & & & & & \\
\hline 20 & & & & & & & & & & & \\
\hline 203 & & & & & & & & & & & \\
\hline & & & & & & & & & & & \\
\hline 2 & & & & & & & & & & & \\
\hline 20 & & & & & & & & & & & \\
\hline & & & & & & & & & & & \\
\hline 208 & & & & & & & & & & & \\
\hline & & & & & & & & & & & \\
\hline 210 & & & & & & & & & & & \\
\hline & & & & & & & & & & & \\
\hline & & & & & & & & & & & \\
\hline 21 & & & & & & & & & & & \\
\hline 214 & .06 & & & & & -8 & & & & & \\
\hline 21 & & & & & & & & & & & \\
\hline & & & & & & & & & & & \\
\hline & & & & & & & & & & & \\
\hline 218 & & & & & & & & & & & \\
\hline & & & & & & & & & & & \\
\hline & & & & & & & & & & & \\
\hline 2 & & & & & & & & & & & \\
\hline 22 & & & & & & & & & & & \\
\hline & & & & & & & & & & & \\
\hline & & & & & & & & & & & \\
\hline 22 & & & & & & & & & & & \\
\hline 22 & & & & & & & & & & & \\
\hline & & & & & & & & & & & \\
\hline 22 & & & & & & & & & & & \\
\hline 229 & 06 & & 4 & & & -8 & 364 & 1 & & & \\
\hline 230 & 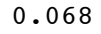 & & & & & & & & & & \\
\hline 2 & & & & & & & & & & & \\
\hline & & & & & & & & & & & \\
\hline 233 & .068 & 0.1225 & .0114 & 0.497 & 7137 & -8 & 0.364 & 26 & 7. & 0.980 & 211 \\
\hline
\end{tabular}




\begin{tabular}{|c|c|c|c|c|c|c|c|c|c|c|c|}
\hline & 100 & 227 & & & 07144 & -86.6 & 364 & 1.126 & 069 & 981 & 2 \\
\hline 35 & & 230 & & 494 & & -86.6 & & & & & \\
\hline 236 & .068 & 233 & 0.0115 & 0.493 & 0.07146 & -86.5 & 365 & 126 & .066 & .983 & 0 \\
\hline 37 & & & & & & & & & & & \\
\hline 38 & & & & & & & & & & & \\
\hline 239 & & & & 198 & & -86.5 & & & & & \\
\hline 240 & .068 & .1244 & & & & & & 1.127 & & .987 & \\
\hline 241 & & & & & & & & & & & \\
\hline 42 & & & & & & & & & & & \\
\hline 43 & & & & & & & & & & & \\
\hline 244 & .068 & & & & & & & & & & \\
\hline 245 & & & & & & & & & & & \\
\hline 246 & & & & & & & & & & & \\
\hline 247 & & & & & & & & & & & \\
\hline 248 & & & & & & & & & & & \\
\hline 249 & & & & & & & & & & & \\
\hline 250 & & & & & & & & & & & \\
\hline 251 & & & & & & & & & & & \\
\hline 252 & & & & & & & & & & & \\
\hline & & & & & & & & & & & \\
\hline 254 & & & & & & & & & & & \\
\hline 255 & .06 & & & & & & & & & & \\
\hline 256 & & & & & & & & & & & \\
\hline 25 & & & & & & & & & & & \\
\hline 258 & & & & & & & & & & & \\
\hline 259 & & & & & & & & & & & \\
\hline 260 & & & & & & & & & & & \\
\hline 20 & & & & & & & & & & & \\
\hline 262 & & & & & & & & & & & \\
\hline 263 & & & & & & & & & & & \\
\hline 264 & & & & & & & & & & & \\
\hline 26 & & & & & & & & & & & \\
\hline 26 & & & & & & & & & & & \\
\hline 26 & & & & & & & & & & & \\
\hline 26 & & & & & & & & & & & \\
\hline 269 & & & & & & & & & & & \\
\hline 270 & & & & & & & & & & & \\
\hline 77 & & & & & & & & & & & \\
\hline 27 & & & & & & & & & & & \\
\hline 21 & & & & & & & & & & & \\
\hline 274 & & & & & & & & & & & \\
\hline 275 & & & & & & & & & & & \\
\hline 21 & & & & & & & & & & & \\
\hline 277 & & & & & & & & & & & \\
\hline & & & & & & & & & & & \\
\hline 27 & & & & & & & & & & & \\
\hline 20 & & & & & & & & & & & \\
\hline 281 & & & & & & 3 & & & & & \\
\hline & & & & & & & & & & & \\
\hline & & & & & & & & & & & \\
\hline 284 & & & & & & -85 & & & & & 65 \\
\hline 285 & & & & & & & & & & & \\
\hline 286 & & & & & & & & & & & \\
\hline 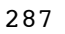 & & & & & & & & & & & \\
\hline & & & & & & & & & & & \\
\hline 289 & & & & & & & & & & & \\
\hline 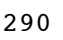 & & & & & & & & & & & \\
\hline 29 & & & & & & & & & & & \\
\hline 29 & & & & & & & & & & & \\
\hline 29 & & & & & & & & & & & \\
\hline 29 & & & & & & & & & & & \\
\hline 29 & & & & & & & & & & & \\
\hline 296 & & & & & & & & & & & \\
\hline 29 & & & & & & & & & & & \\
\hline 29 & & & & & & & & & & & \\
\hline 29 & & & & & & & & & & & \\
\hline 300 & 0.0 & & & 0.524 & & -84.5 & & 1. & & & 79.53 \\
\hline 301 & & & & & & & & & & & \\
\hline ? & & & & & & & & & & & \\
\hline 2 & & & & & & & & & & & \\
\hline 04 & .069 & 86 & 126 & .526 & .08222 & 3 & 0.369 & 37 & 6.9 & 1.079 & 83. \\
\hline
\end{tabular}




\begin{tabular}{|c|c|c|c|c|c|c|c|c|c|c|c|}
\hline & & & & 26 & 28 & 4.3 & 69 & 7 & 1 & 081 & \\
\hline 06 & & & & 527 & & -84.2 & & & & 83 & \\
\hline 307 & .069 & 502 & .0127 & 0.527 & 0.08304 & -84.2 & 0.369 & 1.138 & .936 & .085 & \\
\hline 08 & & & & & 316 & & & & & & \\
\hline 09 & & & & & & & & & & & \\
\hline 10 & 069 & & 27 & 28 & 322 & -84.0 & & & & 91 & \\
\hline 311 & .069 & 523 & .0128 & 0.528 & 0.08387 & -84.0 & 370 & 1.139 & & .092 & \\
\hline 12 & & & & & & & & & & & \\
\hline 13 & & & & & & & & & & & \\
\hline 14 & & & & & & & & & & & \\
\hline 315 & 069 & & & & & & & & & & \\
\hline & & & & & & & & & & & \\
\hline 317 & & & & & & & & & & & \\
\hline 318 & .069 & & & 0.532 & & & & & & & \\
\hline 319 & & & & & & & & & & & \\
\hline 320 & & & & & & & & & & & \\
\hline 321 & & & & & & & & & & & \\
\hline 322 & 060 & & & & & & & & & & \\
\hline 323 & & & & & & & & & & & \\
\hline & & & & & & & & & & & \\
\hline $0 x$ & & & & & & & & & & & \\
\hline 326 & & & & & & & & & & & \\
\hline 327 & & & & & & & & & & & \\
\hline 32 & & & & & & & & & & & \\
\hline 329 & & & & & & & & & & & \\
\hline 330 & & & & & & & & & & & \\
\hline 33 & & & & & & & & & & & \\
\hline 33 & & & & & & & & & & & \\
\hline 333 & & & & & & & & & & & \\
\hline 334 & & & & & & & & & & & \\
\hline 33 & & & & & & & & & & & \\
\hline 336 & & & & & & & & & & & \\
\hline 337 & & & & & & & & & & & \\
\hline 38 & & & & & & & & & & & \\
\hline 33 & & & & & & & & & & & \\
\hline 340 & & & & & & & & & & & \\
\hline & & & & & & & & & & & \\
\hline 342 & & & & & & & & & & & \\
\hline 34 & & & & & & & & & & & \\
\hline 34 & & & & & & & & & & & \\
\hline & & & & & & & & & & & \\
\hline 4 & & & & & & & & & & & \\
\hline 347 & & & & & & & & & & & \\
\hline 348 & & & & & & & & & & & \\
\hline & & & & & & & & & & & \\
\hline 35 & & & & & & & & & & & \\
\hline 35 & & & & & & & & & & & \\
\hline 352 & & & & & & & & & & & \\
\hline & & & & & & & & & & & \\
\hline & & & & & & & & & & & \\
\hline 35 & & & & & & & & & & & \\
\hline 356 & & & & & & & & & & & \\
\hline & & & & & & & & & & & \\
\hline & & & & & & & & & & & \\
\hline 359 & & & & & & & & & & & \\
\hline 36 & & & & & & & & & & & \\
\hline & & & & & & & & & & & \\
\hline 36 & & & & & & & & & & & \\
\hline 363 & .0 & & & & & & & & & & \\
\hline 0 & & & & & & & & & & & \\
\hline & & & & & & & & & & & \\
\hline 36 & & & & & & & & & & & \\
\hline 367 & .0 & & & & & & & & & & \\
\hline & & & & & & & & & & & \\
\hline & & & & & & & & & & & \\
\hline 37 & & & & & & & & & & & \\
\hline 371 & $v$ & & & & & & & & & & 21 \\
\hline 37 & & & & & & & & & & & \\
\hline 3 & & & & & & & & & & & \\
\hline & & & & & & & & & & & \\
\hline 13 & .071 & .2063 & 0.0148 & 0.580 & 0.10353 & & 0.378 & 1.160 & 6.701 & 1.271 & 366.26 \\
\hline
\end{tabular}




\begin{tabular}{|c|c|c|c|c|c|c|c|c|c|c|c|}
\hline 76 & 71 & 75 & 49 & 80 & 29 & 78.9 & 78 & 61 & 696 & 75 & כת \\
\hline 77 & 0.071 & .2088 & 0.0149 & 0.581 & 10448 & 78.8 & 0.378 & 1.161 & 6.691 & 1.279 & 68.80 \\
\hline 8 & 071 & 101 & 150 & 81 & 10508 & 18 & 379 & & 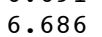 & & \\
\hline 79 & 072 & 14 & 150 & 990 & 28 & & & & & & \\
\hline 380 & 0.072 & 28 & 151 & 0.590 & & -78.5 & 0.379 & & & & \\
\hline 31 & 0.072 & .2141 & 0151 & 0.591 & 10623 & -78 & 0.379 & 1.163 & & 295 & $3 / 3$. \\
\hline 32 & & & & & & & 379 & & & & \\
\hline 33 & .072 & 69 & 152 & 0.592 & & -78.1 & 380 & & & & \\
\hline 84 & 0.072 & 83 & 153 & 0.592 & 0778 & -78.0 & 380 & 65 & 55 & & \\
\hline 35 & & 98 & & 993 & & -77 & 380 & & & & \\
\hline 36 & & & & & & & 380 & & & & \\
\hline 37 & & 27 & & & & -77.6 & 380 & & & & \\
\hline 88 & 0.0 & 42 & 55 & & 62 & -77.5 & .381 & & & & \\
\hline 89 & & & & 99 & & & 381 & & & & \\
\hline 0 & & & & & & & & & & & \\
\hline 91 & 2 & 89 & & & & -77.1 & 381 & & & & \\
\hline 92 & 0.072 & 05 & 57 & & & -77.0 & .382 & & & & \\
\hline 93 & & & & & & & 382 & & & & \\
\hline 4 & & & & & & & & & & & \\
\hline 95 & 0.0 & 54 & & 0.599 & & -76.6 & 382 & & & & \\
\hline 396 & 0070 & & & & & -76.5 & 0.382 & & & & \\
\hline 97 & & & & & & & 83 & & & & \\
\hline 98 & & & & 12 & & .2 & 383 & & & & \\
\hline 99 & & & & & & -76.1 & 383 & & & & \\
\hline 0 & 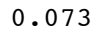 & & & & & & 383 & & & & \\
\hline 01 & & & & & & & 84 & & & & \\
\hline 02 & & & & & & & & & & & \\
\hline 03 & 0073 & & & & & -75.5 & 0.384 & & & & \\
\hline 04 & & & & & & & & & & & \\
\hline 5 & 0.0 & & & & & & 85 & & & & \\
\hline 06 & & & & & & & 85 & & & & \\
\hline 407 & 0 & & & & & -74.9 & 385 & & & & \\
\hline 08 & & & & & & & & & & & \\
\hline 9 & 0.0 & & & & & & 86 & & & & \\
\hline 10 & & & & & & -74.5 & 386 & & & & \\
\hline 411 & & & & & & & & & & & \\
\hline 12 & & & & & & & & & & & \\
\hline 13 & 0 & & & & & -74.0 & 387 & & & & \\
\hline 14 & & & & & & & 387 & & & & \\
\hline 15 & & & & & & & & & & & \\
\hline 16 & & & & & & & & & & & \\
\hline 17 & & & & & & & 388 & & & & \\
\hline 418 & 0.074 & & & & & -7 & 388 & & & & \\
\hline 19 & & & & & & & & & & & \\
\hline 20 & 0 & & & & & -7 & 89 & & & & \\
\hline 21 & 0 & & & & & -72.7 & 89 & & & & \\
\hline 422 & & & & & & 5 & 90 & & & & \\
\hline 23 & & & & & & & & & & & \\
\hline 24 & & & & & & -7 & 90 & & & & \\
\hline 25 & .0 & & & & & -72.0 & 91 & & & & \\
\hline 426 & & & & & & & & & & & \\
\hline 27 & & & & & & & & & & & \\
\hline 28 & & & & & & -7 & 91 & & & & \\
\hline 29 & 0 & & & & & 3 & 92 & & & & \\
\hline 30 & & & & & & & & & & & \\
\hline 1 & & & & & & & & & & & \\
\hline 32 & & & & & & & 93 & & & & \\
\hline 433 & 0.076 & 1 & 0.0 & 0.671 & 66 & -70.6 & 393 & 1. & & & .10 \\
\hline 34 & & & & & & & & & & & \\
\hline 35 & & & & & & & & & & & \\
\hline 36 & & & & & & -70 & 94 & & & & \\
\hline 437 & & & & 0 . & & -69 . & 95 & & & & 52 \\
\hline 38 & & & & & & & & & & & \\
\hline 439 & & & & & & & & & & & \\
\hline 440 & & & & & & 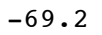 & 96 & & & & \\
\hline 441 & 0 & 5 & 3 & & 2 & -6 & 96 & 1 & & & .06 \\
\hline 42 & 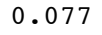 & & & & & & & & & & \\
\hline 443 & & & & & & & & & & & \\
\hline 4 & & & & & & & 98 & & & & \\
\hline 445 & u & 1 & 7 & & 0 & & 98 & 1 & & & 401 \\
\hline 46 & .077 & .3679 & 0.0198 & 0.697 & 0.15351 & -68.1 & 0.398 & 1.220 & 6.179 & 1.698 & 469.4 \\
\hline
\end{tabular}




\begin{tabular}{|c|c|c|c|c|c|c|c|c|c|c|c|}
\hline & & 718 & 199 & 697 & 429 & -67.9 & 399 & 221 & 169 & 707 & 471.1 \\
\hline 448 & 078 & 57 & & 707 & & -67.7 & & 222 & & & \\
\hline 449 & .078 & 797 & 0201 & 0.709 & 5659 & -67.5 & & 1.224 & & .725 & 6 \\
\hline 450 & & 338 & 202 & & & & & & & & \\
\hline 451 & & & & & & & & & & & \\
\hline 452 & .078 & & & 710 & & -66.9 & & & & & \\
\hline 453 & .078 & .3964 & .0206 & 0.711 & 0.16079 & & & 1.229 & & .762 & \\
\hline 454 & .018 & & & & & & & & & & \\
\hline 455 & & & & & & & & & & & \\
\hline 456 & & & & & & & & & & & \\
\hline 457 & .079 & & & & & & & & & & \\
\hline 458 & & & & & & & & & & & \\
\hline 459 & & & & & & & & & & & \\
\hline 460 & .0 & & & 0.729 & & & & & & & \\
\hline 461 & & & & & & & & & & & \\
\hline 462 & & & & & & & & & & & \\
\hline 463 & & & & & & & & & & & \\
\hline 464 & & & & & & & & & & & \\
\hline 465 & & & & & & & & & & & \\
\hline 46 & & & & & & & & & & & \\
\hline 46 & & & & & & & & & & & \\
\hline 468 & 1 & & & & & & & & & & \\
\hline 469 & & & & & & & & & & & \\
\hline 47 & & & & & & & & & & & \\
\hline & & & & & & & & & & & \\
\hline 472 & & & & & & & & & & & \\
\hline 473 & & & & & & & & & & & \\
\hline 47 & & & & & & & & & & & \\
\hline 47 & & & & & & & & & & & \\
\hline 476 & & & & & & & & & & & \\
\hline 47 & & & & & & & & & & & \\
\hline 47 & & & & & & & & & & & \\
\hline 479 & & & & & & & & 1 & & & \\
\hline 48 & & & & & & & & & & & \\
\hline 48 & & & & & & & & & & & \\
\hline 482 & & & & & & & & & & & \\
\hline 483 & & & & & & & & & & & \\
\hline 484 & & & & & & & & & & & \\
\hline 48 & & & & & & & & & & & \\
\hline 48 & & & & & & & & & & & \\
\hline 487 & & & & & & & & & & & \\
\hline 488 & & & & & & & & & & & \\
\hline 489 & & & & & & & & & & & \\
\hline 490 & & & & & & & & & & & \\
\hline & & & & & & & & & & & \\
\hline 49 & & & & & & & & & & & \\
\hline 49 & & & & & & & & & & & \\
\hline 494 & & & & & & -5 & & 1 & & & \\
\hline & & & & & & & & & & & \\
\hline 49 & & & & & & & & & & & \\
\hline 497 & & & & & & -5 & & & & & \\
\hline 498 & & & & 0.849 & & -56.8 & & & & & 30 \\
\hline 49 & & & & & & & & & & & \\
\hline 50 & & & & & & & & & & & \\
\hline 50 & & & & & & -5 & & & & & \\
\hline 50 & & & & & & & & & & & 69 \\
\hline & & & & & & & & & & & \\
\hline 50 & & & & & & & & & & & \\
\hline 50 & & & & & & & & & & & \\
\hline 50 & & & & & & & & & & & \\
\hline-0 & & & & & & & & & & & \\
\hline 50 & & & & & & & & & & & \\
\hline 50 & & & & & & & & & & & \\
\hline & & & & & & & & & & & \\
\hline 51 & & & & & & & & & & & \\
\hline 51 & & & & & & & & & & & \\
\hline 513 & 0.0 & & 0.0 & 0.9 & & & & 1. & & & 8.88 \\
\hline 51 & & & & & & & & & & & \\
\hline 51 & & & & & & & & & & & \\
\hline 51 & & & & & & & & & & & \\
\hline 17 & .092 & 8887 & .0308 & 0.913 & .26184 & .6 & 44 & 1.369 & 5.170 & 2.638 & 610 \\
\hline
\end{tabular}




\begin{tabular}{|c|c|c|c|c|c|c|c|c|c|c|c|}
\hline & & 12 & 310 & & 6396 & -52.4 & 444 & 1.372 & & 657 & \\
\hline 19 & 93 & & & 925 & 91 & 52.2 & & 375 & & & \\
\hline 20 & .093 & .9266 & .0314 & 0.926 & 0.26785 & -52.0 & 0.446 & 1.378 & & .694 & 27.28 \\
\hline 21 & & & & & & & & & & & \\
\hline 22 & 4 & & & & & & & & & & \\
\hline 23 & .094 & 61 & 21 & 36 & 7371 & -51.4 & & .387 & & & \\
\hline 524 & 095 & .9797 & .0323 & 0.947 & 0.27565 & -51.2 & & 1.390 & & & 38.20 \\
\hline 25 & & & & & & & & & & & \\
\hline 26 & & & & & & -50.8 & & & & & \\
\hline 527 & 6 & & & & & -50.6 & & 399 & & & \\
\hline 528 & & & & & & -50 & & & & & \\
\hline & & & & & & & & & & & \\
\hline 530 & & & & & & -50.0 & & & & & \\
\hline 531 & 7 & .0807 & & & & & & & & & \\
\hline 532 & & & & & & & & & & & \\
\hline & & & & & & & & & & & \\
\hline 534 & & & & & & -49.2 & & & & & \\
\hline 535 & 00 & & & & & & & & & & \\
\hline 536 & & & & & & & & & & & \\
\hline J & & & & & & & & & & & \\
\hline & & & & & & -48.4 & & & & & \\
\hline 539 & & & & & & & & & & & \\
\hline 540 & & & & & & & & & & & \\
\hline 54 & & & & & & & & & & & \\
\hline & & & & & & -4 & & & & & \\
\hline 543 & & & & & & & & & & & \\
\hline 44 & & & & & & & & & & & \\
\hline 54 & & & & & & & & & & & \\
\hline 546 & & & & & & & & & & & \\
\hline$n^{-1}$ & & & & & & & & & & & \\
\hline $54 \xi$ & & & & & & & & & & & \\
\hline 54 & & & & & & & & & & & \\
\hline 0 & & & & & & & & & & & \\
\hline 55 & & & & & & & & & & & \\
\hline 55 & & & & & & & & & & & \\
\hline 55 & & & & & & & & & & & \\
\hline 5 & & & & & & & & & & & \\
\hline 5 & & & & & & & & & & & \\
\hline 56 & & & & & & & & & & & \\
\hline 557 & & & & & & & & & & & \\
\hline o & & & & & & & & & & & \\
\hline 5 & & & & & & & & & & & \\
\hline 56 & & & & & & & & & & & \\
\hline 561 & & & & & & & & & & & \\
\hline & & & & & & & & & & & \\
\hline 6 & & & & & & & & & & & \\
\hline 56 & & & & & & & & & & & \\
\hline 565 & & & & & & & & & & & \\
\hline & & & & & & & & & & & \\
\hline 5 & & & & & & & & & & & \\
\hline 56 & & & & & & & & & & & \\
\hline 56 & & & & & & & & & & & \\
\hline & & & & & & & & & & & \\
\hline 57 & & & & & & & & & & & \\
\hline 57 & & & & & & -42 & & & & & \\
\hline 57 & & & & & & & & & & & \\
\hline & & & & & & & & & & & \\
\hline 57 & & & & & & & & & & & \\
\hline 576 & .1 & & & & & & & & & & \\
\hline 57 & & & & & & & & & & & \\
\hline & & & & & & & & & & & \\
\hline 57 & & & & & & & & & & & \\
\hline 580 & 1 & & & & & & & & & & 75 \\
\hline 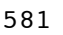 & & & & & & & & & & & \\
\hline & & & & & & & & & & & \\
\hline 58 & & & & & & & & & & & \\
\hline 584 & & & & & & & & & & & \\
\hline 58 & & & & & & & & & & & \\
\hline & & & & & & & & & & & \\
\hline & & & & & & & & & & & \\
\hline 88 & 121 & .3440 & 0.0500 & 1.117 & 32 & $-4 c$ & 0.523 & 1.592 & 3.960 & 4.282 & 861.14 \\
\hline
\end{tabular}




\begin{tabular}{|c|c|c|c|c|c|c|c|c|c|c|c|}
\hline & & & & & & & & & & & \\
\hline ח & & & & & & & & & & & \\
\hline 91 & 122 & 4343 & 0509 & 118 & 39964 & 40 & 527 & 1.600 & & & \\
\hline & & & & & & & & & & & \\
\hline 59 & & & & & & & & & & & \\
\hline 94 & & .5269 & & & 92 & -40.0 & & & & & 7.31 \\
\hline 95 & & & & & & & & & & & \\
\hline & & & & & & & & & & & \\
\hline 97 & & & & & & & & & & & \\
\hline 98 & & & & & & & & & & & \\
\hline 99 & & & & & & & & & & & \\
\hline & & & & & & & & & & & \\
\hline L & & & & & & & & & & & \\
\hline 02 & & & & & & & & & & & \\
\hline 03 & & & & & & & & & & & \\
\hline & & & & & & & & & & & \\
\hline & & & & & & & & & & & \\
\hline 0 & & & & & & & & & & & \\
\hline 607 & & & & & & & & & & & \\
\hline & & & & & & & & & & & \\
\hline 0 & & & & & & & & & & & \\
\hline 610 & & & & & & & & & & & \\
\hline 611 & & & & & & & & & & & \\
\hline & & & & & & & & & & & \\
\hline 61 & & & & & & & & & & & \\
\hline 614 & & & & & & & & & & & \\
\hline 1 & & & & & & & & & & & \\
\hline & & & & & & & & & & & \\
\hline 617 & & & & & & & & & & & \\
\hline 1 & & & & & & & & & & & \\
\hline 61 & & & & & & & & & & & \\
\hline & & & & & & & & & & & \\
\hline & & & & & & & & & & & \\
\hline & & & & & & & & & & & \\
\hline & & & & & & & & & & & \\
\hline 62 & & & & & & & & & & & \\
\hline & & & & & & & & & & & \\
\hline 6 & & & & & & & & & & & \\
\hline & & & & & & & & & & & \\
\hline 62 & & & & & & & & & & & \\
\hline & & & & & & & & & & & \\
\hline & & & & & & & & & & & \\
\hline & & & & & & & & & & & \\
\hline 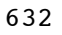 & & & & & & & & & & & \\
\hline & & & & & & & & & & & \\
\hline & & & & & & & & & & & \\
\hline 63 & & & & & & & & & & & \\
\hline & & & & & & & & & & & \\
\hline & & & & & & & & & & & \\
\hline & & & & & & & & & & & \\
\hline 639 & & & & & & & & & & & 86 \\
\hline & & & & & & & & & & & \\
\hline & & & & & & & & & & & \\
\hline 64 & & & & & & & & & & & \\
\hline 64 & & & & & & & & & & & .98 \\
\hline & & & & & & & & & & & \\
\hline & & & & & & & & & & & \\
\hline 04 & & & & & & & & & & & \\
\hline 64 & & & & & & & & & & & \\
\hline & & & & & & & & & & & \\
\hline & & & & & & & & & & & \\
\hline 03 & & & & & & & & & & & \\
\hline 65 & & & & & & & & & & & \\
\hline & & & & & & & & & & & \\
\hline & & & & & & & & & & & \\
\hline & & & & & & & & & & & \\
\hline 65 & & & & & & & & & & & .16 \\
\hline & & & & & & & & & & & \\
\hline & & & & & & & & & & & \\
\hline
\end{tabular}




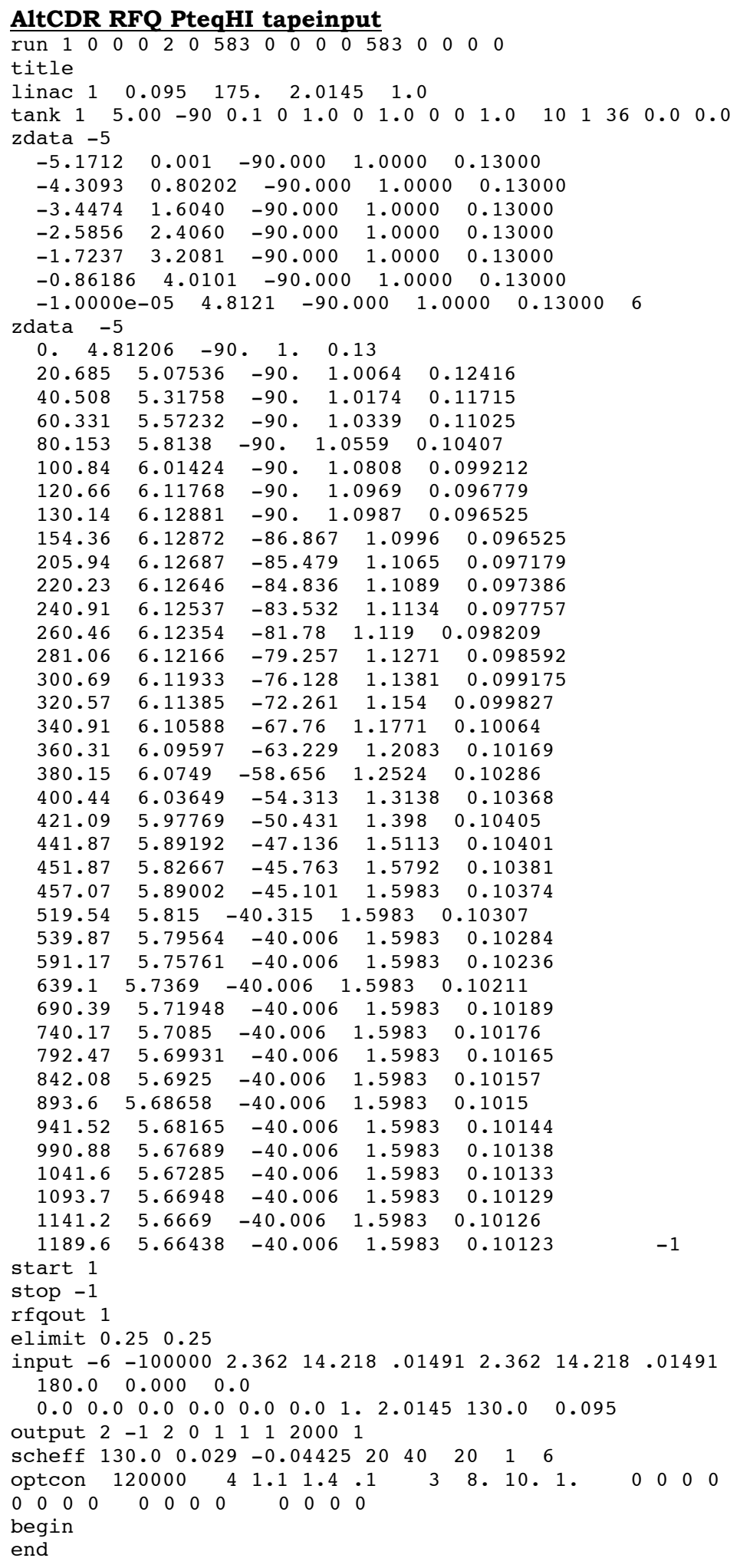

AltCDR RFQ Cell Table 


\begin{tabular}{|c|c|c|c|c|c|c|c|c|c|c|c|}
\hline nc & $V$ & & La & e & capa & pni & d & $\mathrm{m}$ & $b$ & & tl \\
\hline 0 & 0.130 & 0950 & 0.0101 & 0.000 & 0.00000 & -90.0 & 45.090 & 1.000 & 0.001 & 0.000 & 0.0 \\
\hline 1 & 0.130 & 950 & 0.0101 & 0.000 & 0000 & -90.0 & 1.592 & 1.000 & .802 & & 列 \\
\hline 2 & 0.130 & .0950 & .0101 & 0.000 & 0.00000 & -90.0 & 1.126 & 1.000 & 1.604 & 0.862 & 1.72 \\
\hline 3 & & & & 0.000 & 000 & & & & & & \\
\hline 4 & .130 & 50 & 0.0101 & 0.000 & 0.00000 & -90.0 & & & & & .45 \\
\hline 5 & .130 & 50 & 01 & 0.000 & 000 & -90.0 & & 1.000 & 10 & & .31 \\
\hline 6 & 0.130 & 0.0950 & 0.0101 & 0.000 & 0.00000 & -90.0 & 0.650 & 1.000 & 4.812 & 0.862 & 5.17 \\
\hline 7 & 130 & 50 & & 000 & 000 & & & & & & \\
\hline 8 & .130 & & & & & & & & & & .89 \\
\hline 9 & .129 & 50 & 0.0101 & 0.004 & 024 & -90.0 & & 1.0 & & & 7.76 \\
\hline 10 & .129 & & & & & & & & & & \\
\hline 11 & .129 & & & & & & & & & & \\
\hline 12 & 0.129 & & & & & -90.0 & & & & & \\
\hline 13 & 0.1 & 50 & & 0.0 & & -90.0 & & & & & 1.20 \\
\hline 14 & 0120 & & & & & & & & & & \\
\hline 15 & 120 & & & & & & & & & & \\
\hline 16 & 0.128 & & & & & -90.0 & & & & & \\
\hline 17 & 0.127 & & & & & & & & & & 4.65 \\
\hline 18 & .127 & & & & & & & & & & \\
\hline 19 & . & & & & & & & & & & \\
\hline 20 & 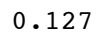 & & & & & & & & & & \\
\hline 21 & 0.126 & 0 . & & 0 & & & & & & & \\
\hline 22 & .1 & & & & & & & & & & \\
\hline 23 & & & & & & & & & & & \\
\hline 24 & & & & & & & & & & & \\
\hline 25 & $0_{1}$ & & & & & & & & & & \\
\hline 26 & & & & & & & & & & & \\
\hline 27 & & & & & & & & & & & \\
\hline 28 & 0 & & & & & -9 & & 1. & & & 13 \\
\hline 29 & 12 & & & & & & & & & & \\
\hline 30 & 0 & & & & & & & & & & \\
\hline 31 & 0.1 & & & & & & & & & & \\
\hline 32 & 0.124 & & & 0.0 & & -9 & 13 & 1.0 & & & 7.58 \\
\hline 33 & 0 & & & & & & & & & & \\
\hline 34 & .1 & & & & & & & & & & \\
\hline 35 & & & & & & & & & & & \\
\hline 36 & 122 & & & 0.0 & & -9 & & 1. & & & 03 \\
\hline 37 & & & & & & & & & & & \\
\hline 38 & .1 & & & & & & & & & & \\
\hline 39 & .1 & & & & & & & & & & \\
\hline 40 & & & & & & & & & & & \\
\hline 41 & & & & & & & & & & & \\
\hline 42 & 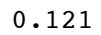 & & & & & & & & & & \\
\hline 43 & 0.1 & & 1 & & 55 & -90 & & 1.0 & & & 37.06 \\
\hline 44 & & & & & & & & & & & \\
\hline 45 & & & & & & & & & & & \\
\hline 46 & & & & & & & & & & & 65 \\
\hline 47 & 0.1 & & & & & -9 & & 1 & & & .51 \\
\hline 18 & & & & & & & & & & & \\
\hline 49 & & & & & & & & & & & \\
\hline 50 & 0 & & & & & & & & & & 09 \\
\hline 51 & 0.1 & 51 & 1 & 0 & & -90.0 & & 1. & & & .95 \\
\hline 52 & & & & & & & & & & & \\
\hline 53 & & & & & & & & & & & \\
\hline 54 & & & & & & & & & & & \\
\hline 55 & & & & & & & & & & & \\
\hline 56 & & & & & & & & & & & \\
\hline 57 & & & & & & & & & & & \\
\hline 58 & 0.1 & & 0.0 & & & -90.0 & & & & & .99 \\
\hline 59 & .1 & & & & & & & & & & .85 \\
\hline 60 & & & & & & & & & & & \\
\hline 61 & & & & & & & & & & & \\
\hline 62 & 0.1 & & & & & -90 & & & & & 3.44 \\
\hline 63 & & & & & & & & & & & \\
\hline 64 & & & & & & & & & & & \\
\hline 65 & & & & & & & & & & & \\
\hline 66 & 0.1 & 0.0952 & 0.0101 & 0.109 & 0.00 & -90 & 0.562 & 1.027 & 5.462 & 0 . & 56.88 \\
\hline 67 & -2 & & & & 0. & & & & & & \\
\hline & & & & & & & & & & & \\
\hline 69 & .112 & 52 & 1 & 0.117 & 0.00905 & -90.0 & 0.558 & 1.029 & 5.495 & 0.863 & 59.47 \\
\hline
\end{tabular}




\begin{tabular}{|c|c|c|c|c|c|c|c|c|c|c|c|}
\hline 70 & & 952 & 01 & 22 & 0941 & 0.0 & 556 & 030 & 506 & 863 & . \\
\hline 71 & & 52 & 0101 & & 0.00944 & 0.0 & & .030 & & & \\
\hline 72 & .111 & 52 & 0101 & 126 & & -90.0 & & & & & \\
\hline 73 & .111 & 52 & & & & -90.0 & & 1.032 & .539 & & 2.92 \\
\hline 74 & & & & & & & & & & & \\
\hline 75 & & & & & & & & & & & \\
\hline 76 & 0 & & & & & .0 & & & & & 0 \\
\hline 77 & 110 & .0952 & & & & & & & & & \\
\hline 78 & & & & & & & & & & & \\
\hline 79 & & & & & & & & & & & \\
\hline 80 & 9 & & & & & -9 & & & & & \\
\hline 81 & & & & & & & & & & & \\
\hline 82 & & & & & & & & & & & \\
\hline 83 & & & & & & & & & & & \\
\hline 84 & & & & & & & & & & & \\
\hline 85 & & & & & & & & & & & \\
\hline 86 & & & & & & & & & & & \\
\hline 87 & & & & & & & & & & & \\
\hline 88 & 1 & & & & & & & & & & \\
\hline 89 & & & & & & & & & & & \\
\hline 90 & & & & & & & & & & & \\
\hline 91 & & & & & & & & & & & \\
\hline 92 & .106 & & & & & & & & & & \\
\hline 93 & & & & & & & & & & & \\
\hline 94 & & & & & & & & & & & \\
\hline 95 & & & & & & & & & & & \\
\hline 96 & & & & & & & & & & & \\
\hline 97 & & & & & & & & & & & \\
\hline 98 & & & & & & & & & & & \\
\hline 99 & & & & & & & & & & & \\
\hline 100 & & & & & & & & & & & \\
\hline 01 & & & & & & & & & & & \\
\hline 02 & & & & & & & & & & & \\
\hline 103 & & & & & & & & & & & 77 \\
\hline 104 & & & & & & & & & & & \\
\hline 05 & & & & & & & & & & & \\
\hline 106 & & & & & & & & & & & 36 \\
\hline 107 & & & & & & & & & & & 2.22 \\
\hline 108 & & & & & & & & & & & \\
\hline 09 & & & & & & & & & & & \\
\hline 10 & & & & & & & & & & & \\
\hline & & & & & & & & & & & \\
\hline & & & & & & & & & & & \\
\hline & & & & & & & & & & & \\
\hline 114 & 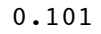 & & & & & & & & & & 8.25 \\
\hline 115 & & & & & & & & & & & \\
\hline & & & & & & & & & & & \\
\hline & & & & & & & & & & & \\
\hline 118 & 0 & & & & & & & & & & 70 \\
\hline & & & & & & & & & & & \\
\hline 1 & & & & & & & & & & & \\
\hline 121 & & & & & & & & & & & \\
\hline 122 & 0 & & & & & & & & & & 15 \\
\hline & & & & & & & & & & & \\
\hline & & & & & & & & & & & \\
\hline 125 & & & & & & & & & & & \\
\hline 126 & & & & & & & & & & & \\
\hline & & & & & & & & & & & \\
\hline 12 & & & & & & & & & & & \\
\hline 129 & 0.0 & & & 0.3 & & -9 & & & & & .18 \\
\hline 130 & 0 & & & & & & & & & & .04 \\
\hline & & & & & & & & & & & \\
\hline 13 & & & & & & & & & & & \\
\hline 133 & 0.0 & & & & & & & & & & \\
\hline 13 & & & & & & & & & & & \\
\hline 1 & & & & & & & & & & & \\
\hline & & & & & & & & & & & \\
\hline 137 & $.0 s$ & & 0 & 0.3 & 0. & & & 1.0 & & & .08 \\
\hline 138 & .0 & & & 0 . & & & & & & & \\
\hline & & & & & & & & & & & \\
\hline 140 & .097 & 55 & & 0.386 & & & 79 & 1 & 91 & 0. & 120 \\
\hline
\end{tabular}




\begin{tabular}{|c|c|c|c|c|c|c|c|c|c|c|c|}
\hline & 1 & 55 & 101 & 87 & 54 & 0.0 & 78 & 3 & 095 & 864 & 21.5 \\
\hline 142 & & & & 92 & & 0 & & & & & \\
\hline 143 & & & 101 & & & .0 & & & & & \\
\hline 144 & .097 & & & 0.401 & & -90.0 & & & & & 24.11 \\
\hline 145 & & & & & & & & & & & \\
\hline 146 & & & & & & & & & & & \\
\hline 147 & & & & & & -90.0 & & & & & \\
\hline 148 & .097 & & & & & & & & & & 7.56 \\
\hline 149 & $0^{0}$ & & & & & & & & & & \\
\hline 150 & & & & & & & & & & & \\
\hline 151 & & & & & & .0 & & & & & \\
\hline 152 & & & & & & & & & & & \\
\hline 153 & & & & & & & & & & & \\
\hline 154 & & & & & & & & & & & \\
\hline 155 & & & & & & & & & & & \\
\hline 156 & & & & & & & & & & & \\
\hline 157 & & & & & & & & & & & \\
\hline 158 & & & & & & & & & & & \\
\hline 159 & & & & & & & & & & & \\
\hline 160 & & & & & & & & & & & \\
\hline 161 & & & & & & & & & & & \\
\hline 162 & & & & & & & & & & & \\
\hline 163 & .09 & & & & & & & & & & \\
\hline 164 & & & & & & & & & & & \\
\hline 165 & & & & & & & & & & & \\
\hline 16 & & & & & & & & & & & \\
\hline 167 & & & & & & & & & & & \\
\hline 168 & & & & & & & & & & & \\
\hline 16 & & & & & & & & & & & \\
\hline 170 & & & & & & & & & & & \\
\hline 171 & & & & & & & & & & & \\
\hline 172 & & & & & & & & & & & \\
\hline 17 & & & & & & & & & & & \\
\hline 174 & & & & & & & & & & & \\
\hline 1 & & & & & & & & & & & \\
\hline 17 & & & & & & & & & & & \\
\hline 17 & & & & & & & & & & & \\
\hline 178 & & & & & & & & & & & \\
\hline 1 & & & & & & & & & & & \\
\hline 1 & & & & & & & & & & & \\
\hline 18 & & & & & & & & & & & \\
\hline 182 & & & & & & & & & & & \\
\hline 1 & & & & & & & & & & & \\
\hline & & & & & & & & & & & \\
\hline 185 & & & & & & -8 & & & & & \\
\hline 186 & & & & & & & & & & & \\
\hline & & & & & & & & & & & \\
\hline & & & & & & & & & & & \\
\hline 189 & & & & & & 8 & & & & & .08 \\
\hline & & & & & & & & & & & \\
\hline & & & & & & & & & & & \\
\hline 192 & & & & & & & & & & & 72 \\
\hline 193 & & & & & & 7 & & & & & 60 \\
\hline & & & & & & & & & & & \\
\hline & & & & & & & & & & & \\
\hline 19 & & & & & & & & & & & \\
\hline 197 & & & & & & & & & & & \\
\hline & & & & & & & & & & & \\
\hline 19 & & & & & & & & & & & \\
\hline 200 & & & & & & -86.5 & & & & & .80 \\
\hline 201 & & & & & & & & & & & \\
\hline 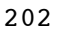 & & & & & & & & & & & \\
\hline 20 & & & & & & & & & & & \\
\hline 204 & • & & & & & & & & & & .3 \\
\hline 20 & & & & & & & & & & & \\
\hline & & & & & & & & & & & \\
\hline 20 & & & & & & & & & & & \\
\hline 208 & .0 & & 0 & & & -8 & & 1. & & & .95 \\
\hline . & 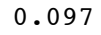 & & & & & & & & & & \\
\hline & & & & & & & & & & & \\
\hline 211 & .097 & & & & & .2 & & & 28 & 3 & \\
\hline
\end{tabular}




\begin{tabular}{|c|c|c|c|c|c|c|c|c|c|c|c|}
\hline 212 & & & 06 & 34 & 64 & -86.2 & 74 & 03 & 128 & 4 & 55 \\
\hline 3 & 097 & .1049 & 0.0106 & 0.436 & 0.04077 & -86.2 & 0.474 & 1.103 & 6.128 & 0.906 & $184 \triangle 15$ \\
\hline 4 & & 052 & 106 & & 04083 & 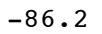 & 474 & & & & \\
\hline 15 & 097 & 54 & 106 & 0.437 & 89 & & & & & & \\
\hline 16 & 0.097 & & 6 & 138 & 5 & -86.1 & 0.474 & 03 & & & $=0$ \\
\hline 17 & 097 & .1060 & .0106 & 438 & 0.04101 & 86.1 & 0.474 & 1.103 & 128 & 0 & 000 \\
\hline 8 & & 063 & 106 & & & & 474 & & & & \\
\hline 19 & 097 & 065 & 0107 & 0.439 & 04149 & 86.1 & 474 & & 128 & & \\
\hline 20 & 0.097 & 68 & 107 & 0.440 & 62 & -86.0 & 0.474 & 1.104 & 128 & & \\
\hline 21 & & 071 & & & & 6 & 474 & & & & \\
\hline 2 & & & & & & 36.0 & 474 & & & & \\
\hline 23 & & 77 & & & 30 & -86.0 & 474 & & & & \\
\hline 24 & 0.0 & 80 & 0. & & 93 & -85.9 & .474 & & 6.127 & & \\
\hline 25 & & 3 & & & & -85.9 & 474 & & & & \\
\hline 6 & & & & & & & & & & & \\
\hline 27 & 7 & & 08 & 0.445 & 47 & -85.9 & 0.474 & & 27 & & 0 \\
\hline 228 & 0.0 & 92 & .08 & & & -85.8 & 0.474 & & & & \\
\hline 229 & & & & & & & & & & & \\
\hline 0 & & & & & & & & & & & \\
\hline 231 & 0 & 01 & 08 & & 8 & -85.8 & 0.474 & & & & \\
\hline 232 & 0 & & & & & $-85,7$ & & & & & \\
\hline 233 & & & & & & & & & & & \\
\hline 34 & & & & & & & & & & & \\
\hline 35 & & & & & & & 474 & & & & \\
\hline 36 & & & & & & & & & & & \\
\hline 37 & & & & & & & & & & & \\
\hline 38 & & & & & & & & & & & \\
\hline 239 & 0.097 & & & & & -85.6 & 0.474 & & 27 & & \\
\hline 40 & & & & & & & & & & & \\
\hline 41 & 0.0 & & & & & -63 & 74 & & & & \\
\hline 42 & & & & & & 85.5 & 74 & & & & \\
\hline 243 & 0 & & & & & -85.4 & 474 & & 27 & & \\
\hline 44 & & & & & & & & & & & \\
\hline 45 & 0.0 & & & & & -0. & & & & & \\
\hline 246 & & & & & 31 & 85.3 & 474 & 1. & & & \\
\hline 247 & & & & & & & & & & & \\
\hline 48 & & & & & & & & & & & \\
\hline 49 & .0 & & & & & -85.2 & 74 & 1 & 27 & & \\
\hline 50 & & & & & & 1 & & & & & \\
\hline 251 & & & & & & & & & & & \\
\hline 52 & & & & & & & & & & & \\
\hline 53 & & & & & & 85.0 & & & & & \\
\hline 254 & 0 & & & & & -85 & 74 & & 27 & & 54 \\
\hline 55 & & & & & & & & & & & \\
\hline 56 & & & & & & & & & & & \\
\hline 57 & 0 & & & & & -84.8 & 74 & & & & \\
\hline 258 & & & & & & & & & & & 38 \\
\hline 59 & & & & & & & & & & & \\
\hline 50 & & & & & & & & & & & \\
\hline 61 & 0.0 & & 14 & & & -84.6 & 73 & 1 & & & \\
\hline 262 & & & & & & & & & & & \\
\hline 53 & & & & & & & & & & & \\
\hline 64 & & & & & & -8 & 73 & & 6 & & 20 \\
\hline 65 & 0 & & & & & -84.3 & & & & & 18 \\
\hline 66 & & & & & & & & & & & 10 \\
\hline 7 & & & & & & & & & & & \\
\hline 98 & & & & & & -84.2 & & & & & \\
\hline 69 & 0.098 & 5 & 0.0 & 0.485 & 9 & -84.1 & 0.473 & 1.1 & 26 & & .10 \\
\hline 70 & & & & & & & & & & & \\
\hline 271 & & & & & & & & & & & \\
\hline 72 & & & & & & -8 & & & & & \\
\hline 273 & 0.0 & 5 & 16 & 0.490 & 7 & -83.8 & 73 & 1. & & & .05 \\
\hline 74 & & & & & & & & & & & \\
\hline $7 x$ & & & & & & & & & & & \\
\hline 76 & & & & & & -83 & & & & & \\
\hline 277 & 0.0 & 0.1287 & 0117 & 4 & 5 & -83.6 & 0.473 & 3 & 25 & & 5.03 \\
\hline 78 & . & & & & & & & & & & \\
\hline 279 & & & & & & & & & & & \\
\hline 80 & & & & & & & & & & & \\
\hline 281 & u & 9 & 8 & & 0.05 & & 3 & 10 & 5 & & 49.05 \\
\hline 82 & 0.098 & .1315 & 0.0118 & 0.504 & .05201 & -83.2 & 0.473 & 1. & 6.125 & 1.014 & -0 \\
\hline
\end{tabular}




\begin{tabular}{|c|c|c|c|c|c|c|c|c|c|c|c|}
\hline J & 8 & 321 & 119 & 02 & 20 & .1 & 73 & 5 & 125 & .016 &. \\
\hline 84 & & 327 & & 503 & 232 & 0 & & & & & \\
\hline 85 & 8 & 33 & & 504 & & 9 & & & & & \\
\hline 286 & .098 & & & 0.510 & 0.05303 & -82.8 & 473 & & & 023 & \\
\hline 87 & & & & & & & & & & & \\
\hline 88 & & & & & & & & & & & \\
\hline 289 & 8 & & & 13 & & -82.5 & & & & & \\
\hline 290 & .098 & 366 & & 0.510 & & -82.4 & 0.473 & & & & \\
\hline 91 & & & & & & & & & & & \\
\hline 92 & & & & & & & & & & & \\
\hline 293 & 8 & & & & & -8 & & & & & \\
\hline 294 & & & & & & & & & & & \\
\hline 295 & & & & & & & & & & & \\
\hline 96 & & & & & & & & & & & \\
\hline 297 & & & & & & & & & & & \\
\hline 298 & & & & & & & & & & & \\
\hline 299 & & & & & & & & & & & \\
\hline 300 & & & & & & & & & & & \\
\hline 301 & & & & & & & & & & & \\
\hline 302 & & & & & & & & & & & \\
\hline 303 & & & & & & & & & & & \\
\hline 304 & & & & & & & & & & & \\
\hline 305 & .09 & & & & & & & & & & \\
\hline 306 & & & & & & & & & & & \\
\hline 30 & & & & & & & & & & & \\
\hline 30 & & & & & & & & & & & \\
\hline 309 & & & & & & & & & & & \\
\hline 310 & & & & & & & & & & & \\
\hline & & & & & & & & & & & \\
\hline 312 & & & & & & & & & & & \\
\hline 313 & & & & & & & & & & & \\
\hline 314 & & & & & & & & & & & \\
\hline 31 & & & & & & & & & & & \\
\hline 316 & & & & & & & & & & & \\
\hline ? & & & & & & & & & & & \\
\hline 31 & & & & & & & & & & & \\
\hline 31 & & & & & & & & & & & \\
\hline 320 & & & & & & & & & & & \\
\hline & & & & & & & & & & & \\
\hline 32 & & & & & & & & & & & \\
\hline 32 & & & & & & & & & & & \\
\hline 324 & & & & & & & & & & & \\
\hline 3 & & & & & & & & & & & \\
\hline 32 & & & & & & & & & & & \\
\hline 327 & & & & & & -7 & & & & & .53 \\
\hline 328 & & & & & & & & & & & \\
\hline & & & & & & & & & & & \\
\hline & & & & & & & & & & & \\
\hline 331 & & & & & & & & & & & \\
\hline & & & & & & & & & & & \\
\hline 3 & & & & & & & & & & & \\
\hline 334 & & & & & & & & & & & \\
\hline 335 & .0 & & & & & & & & & & \\
\hline & & & & & & & & & & & \\
\hline & & & & & & & & & & & \\
\hline 33 & & & & & & & & & & & \\
\hline 339 & & & & & & & & & & & \\
\hline 34 & & & & & & & & & & & \\
\hline 34 & & & & & & & & & & & \\
\hline 342 & & & & & & & & & & & \\
\hline 343 & & & & & & & & & & & \\
\hline ? & & & & & & & & & & & \\
\hline 34 & & & & & & & & & & & \\
\hline 346 & 0 & & & & & & & & & & .59 \\
\hline 7 & & & & & & & & & & & \\
\hline ? & & & & & & & & & & & \\
\hline 34 & & & & & & & & & & & \\
\hline 350 & .1 & & 0 & 0 . & 0. & -72.0 & & & & & 26.73 \\
\hline 35 & & & & 0.6 & & & & & & & \\
\hline & & & & & & & & & & & \\
\hline 353 & & & & & & & 59 & & 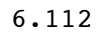 & 1. & 30 \\
\hline
\end{tabular}




\begin{tabular}{|c|c|c|c|c|c|c|c|c|c|c|c|}
\hline & & 277 & & 690 & 09219 & 0. & & & & & 332.00 \\
\hline 355 & & 305 & & 698 & & -70.6 & & & & & \\
\hline 356 & 100 & .2334 & 0158 & 0.700 & 0.09477 & -70.3 & 468 & 1.164 & & .351 & \\
\hline 57 & & & & 707 & & & & & & & \\
\hline 58 & & & & & & & & & & & \\
\hline 359 & 0 & & 161 & & 59 & -69.4 & & 169 & & & \\
\hline 360 & .100 & .2459 & .0162 & 0.720 & 0.09984 & -69.1 & & & & .387 & \\
\hline 361 & & & & 6 & & & & & & & \\
\hline 62 & & & & & & & & & & & \\
\hline 363 & & & & & & & & & & & \\
\hline 364 & & .2598 & & & & -67.8 & & & & & \\
\hline 365 & & & & & & & & & & & \\
\hline 366 & & & & & & & & & & & \\
\hline 367 & & & & & & & & & & & \\
\hline 368 & & & & & & & & & & & \\
\hline 369 & & & & & & 6 & & & & & \\
\hline 370 & & & & & & & & & & & \\
\hline 371 & 1 & & & & & & & & & & \\
\hline 372 & & & & & & & & & & & \\
\hline & & & & & & & & & & & \\
\hline 374 & & & & & & -64.4 & & & & & \\
\hline 375 & .102 & & & & & & & & & & \\
\hline 376 & & & & & & & & & & & \\
\hline 37 & & & & & & & & & & & \\
\hline & & & & & & & & & & & \\
\hline 379 & & & & & & & & & & & \\
\hline 380 & & & & & & & & & & & \\
\hline 38 & & & & & & & & & & & \\
\hline 382 & & & & & & & & & & & \\
\hline 38 & & & & & & & & & & & \\
\hline 38 & & & & & & & & & & & \\
\hline 38 & & & & & & & & & & & \\
\hline 386 & & & & & & & & & & & \\
\hline & & & & & & & & & & & \\
\hline 38 & & & & & & & & & & & \\
\hline 389 & & & & & & & & & & & \\
\hline 390 & & & & & & -58.3 & & & & & \\
\hline 20 & & & & & & & & & & & \\
\hline 39 & & & & & & & & & & & \\
\hline 39 & & & & & & & & & & & \\
\hline A & & & & & & & & & & & \\
\hline 39 & & & & & & & & & & & \\
\hline 396 & & & & & & & & & & & \\
\hline 397 & & & & & & & & & & & \\
\hline & & & & & & & & & & & \\
\hline 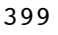 & & & & & & & & & & & \\
\hline 40 & & & & & & & & & & & \\
\hline 401 & 0 & & & & & & & 1 & & & \\
\hline & & & & & & & & & & & \\
\hline 40 & & & & & & & & & & & \\
\hline 404 & & & & & & -52 & & & & & \\
\hline 405 & 1 & & & 1.170 & & -52.4 & & & & & 5.75 \\
\hline 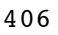 & & & & & & & & & & & \\
\hline 40 & & & & & & & & & & & \\
\hline 408 & & & & & & & & & & & \\
\hline 409 & & & & & & & & & & & \\
\hline & & & & & & & & & & & \\
\hline 41 & & & & & & & & & & & \\
\hline 41 & & & & & & & & & & & \\
\hline 41 & & & & & & & & & & & \\
\hline 41 & & & & & & & & & & & \\
\hline 41 & & & & & & & & & & & \\
\hline 41 & & & & & & & & & & & \\
\hline 41 & & & & & & & & & & & \\
\hline 41 & & & & & & & & & & & \\
\hline 41 & & & & & & & & & & & \\
\hline 420 & 0.1 & & 0.0 & 1.425 & & -46.7 & & & & & 50.05 \\
\hline 42 & & & & & & & & & & & \\
\hline 42 & & & & & & & & & & & \\
\hline 42 & & & & & & & & & & & \\
\hline 24 & .104 & 3932 & .0308 & 1.486 & .37699 & 3 & 0.399 & 1.591 & 5.867 & 2. & $6 r$ \\
\hline
\end{tabular}




\begin{tabular}{|c|c|c|c|c|c|c|c|c|c|c|c|}
\hline & & 164 & 312 & & 8147 & -45.0 & 397 & 1.598 & 889 & 676 & 463.00 \\
\hline 426 & & 99 & & 69 & & -44 & & & & & \\
\hline 427 & .104 & 535 & 0320 & 454 & 0.38333 & -44.6 & 397 & 1.598 & 83 & 744 & 2 \\
\hline 428 & & 372 & 324 & & & & 397 & & & & \\
\hline 429 & & & & & & & & & & & \\
\hline 430 & 4 & & & & & -44.0 & & & & & \\
\hline 431 & & .0591 & .0336 & & 0.38689 & & & & & .877 & 79.65 \\
\hline 432 & & & & & & & & & & & \\
\hline 433 & & & & & & & & & & & \\
\hline 434 & & & & & & & & & & & \\
\hline 435 & & & & & & & & & & & \\
\hline 436 & & & & & & & & & & & \\
\hline 437 & & & & & & & & & & & \\
\hline 438 & & & & & & & & & & & \\
\hline 439 & & & & & & & & & & & \\
\hline 440 & & & & & & & & & & & \\
\hline 441 & & & & & & & & & & & \\
\hline 442 & & & & & & & & & & & \\
\hline 443 & & & & & & & & & & & \\
\hline 444 & & & & & & & & & & & \\
\hline 44 & & & & & & & & & & & \\
\hline 446 & .103 & & & & & & & & & & \\
\hline 447 & & & & & & & & & & & \\
\hline 448 & & & & & & & & & & & \\
\hline & & & & & & & & & & & \\
\hline 450 & & & & & & & & & & & \\
\hline 451 & & & & & & & & & & & \\
\hline 45 & & & & & & & & & & & \\
\hline 453 & & & & & & & & & & & \\
\hline 454 & & & & & & & & & & & \\
\hline 455 & & & & & & & & & & & \\
\hline 456 & & & & & & & & & & & \\
\hline 457 & & & & & & & & & & & \\
\hline 45 & & & & & & & & & & & \\
\hline 45 & & & & & & & & & & & \\
\hline 460 & & & & & & & & & & & \\
\hline 461 & & & & & & & & & & & \\
\hline 462 & & & & & & & & & & & \\
\hline 46 & & & & & & & & & & & \\
\hline 46 & & & & & & & & & & & \\
\hline 465 & & & & & & & & & & & \\
\hline 46 & & & & & & & & & & & \\
\hline 46 & & & & & & & & & & & \\
\hline 468 & & & & & & & & & & & \\
\hline 469 & & & & & & & & & & & \\
\hline 470 & & & & & & & & & & & \\
\hline 47 & & & & & & & & & & & \\
\hline 472 & & & & & & -4 & & & & & 81 \\
\hline 47 & & & & & & & & & & & \\
\hline 47 & & & & & & & & & & & \\
\hline 47 & & & & & & & & & & & \\
\hline 476 & & & & & & & & & & & 23 \\
\hline 477 & & & & & & & & & & & \\
\hline 47 & & & & & & & & & & & \\
\hline 47 & & & & & & & & & & & \\
\hline 480 & & & & & & & & & & & \\
\hline 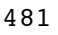 & & & & & & & & & & & \\
\hline 48 & & & & & & & & & & & \\
\hline 48 & & & & & & & & & & & \\
\hline 48 & & & & & & & & & & & \\
\hline 48 & & & & & & & & & & & \\
\hline 48 & & & & & & & & & & & \\
\hline 487 & & & & & & & & & & & \\
\hline 48 & & & & & & & & & & & \\
\hline & & & & & & & & & & & \\
\hline 49 & & & & & & & & & & & \\
\hline 491 & 0. & 2 & & & & & & & & & .20 \\
\hline 49 & & & & & & & & & & & \\
\hline A & & & & & & & & & & & \\
\hline & & & & & & & & & & & \\
\hline 95 & .102 & .7094 & .0537 & 0.908 & 0.40938 & & 0.397 & 1.598 & 5.714 & 4.598 & 722 \\
\hline
\end{tabular}




\begin{tabular}{|c|c|c|c|c|c|c|c|c|c|c|c|}
\hline & & & 539 & & 40953 & 0.0 & 397 & 598 & 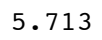 & 4.620 & \\
\hline 497 & & & 542 & & 967 & -40.0 & & & & & \\
\hline 498 & 102 & .7878 & 0544 & 0.897 & 0.40981 & -40.0 & 0.397 & 1.598 & .710 & 4.664 & 28 \\
\hline 499 & & & & 392 & 994 & & 397 & & & & \\
\hline 00 & & & & & & & & & & & \\
\hline 501 & & & & & 021 & -40.0 & & & & & \\
\hline 502 & 102 & .8924 & .0555 & 0.880 & 0.41034 & -40.0 & 0.397 & & 5.707 & .750 & \\
\hline 503 & & & & & & & & & & & \\
\hline 504 & & & & & & & & & & & \\
\hline 505 & & & & & & & & & & & \\
\hline 506 & & .9970 & & & & & & & & & \\
\hline 507 & & & & & & & & & & & \\
\hline 508 & & & & & & & & & & & \\
\hline 509 & & & & & & & & & & & \\
\hline 510 & $10 ?$ & & & & & & & & & & \\
\hline 511 & & & & & & & & & & & \\
\hline 512 & & & & & & & & & & & \\
\hline 513 & 2 & & & & & & & & & & \\
\hline 514 & & & & & & & & & & & \\
\hline & & & & & & & & & & & \\
\hline 516 & & & & & & & & & & & \\
\hline 517 & 1 & & & & & & & & & & \\
\hline 518 & & & & & & & & & & & \\
\hline 51 & & & & & & & & & & & \\
\hline & & & & & & & & & & & \\
\hline 521 & & & & & & & & & & & \\
\hline 522 & & & & & & & & & & & \\
\hline 52 & & & & & & & & & & & \\
\hline 524 & & & & & & & & & & & \\
\hline 52 & & & & & & & & & & & \\
\hline 52 & & & & & & & & & & & \\
\hline 52 & & & & & & & & & & & \\
\hline 528 & & & & & & & & & & & \\
\hline-2 & & & & & & & & & & & \\
\hline 53 & & & & & & & & & & & \\
\hline 531 & & & & & & & & & & & \\
\hline 532 & & & & & & & & & & & \\
\hline 533 & & & & & & & & & & & \\
\hline 53 & & & & & & & & & & & \\
\hline 53 & & & & & & & & & & & \\
\hline 53 & & & & & & & & & & & \\
\hline 3 & & & & & & & & & & & \\
\hline 53 & & & & & & & & & & & \\
\hline 539 & & & & & & & & & & & \\
\hline & & & & & & & & & & & \\
\hline 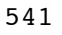 & & & & & & & & & & & \\
\hline 542 & & & & & & & & & & & \\
\hline 543 & 0 & & & & & & & & & & \\
\hline & & & & & & & & & & & \\
\hline 5 & & & & & & & & & & & \\
\hline 546 & & & & & & & & & & & \\
\hline 547 & & & & & & -4 & & & & & 17 \\
\hline 548 & & & & & & & & & & & \\
\hline- & & & & & & & & & & & \\
\hline 55 & & & & & & & & & & & \\
\hline 55 & & & & & & & & & & & \\
\hline & & & & & & & & & & & \\
\hline 55 & & & & & & & & & & & \\
\hline 55 & & & & & & & & & & & \\
\hline & & & & & & & & & & & \\
\hline & & & & & & & & & & & \\
\hline 55 & & & & & & & & & & & \\
\hline 55 & & & & & & & & & & & \\
\hline 55 & & & & & & & & & & & \\
\hline & & & & & & & & & & & \\
\hline 56 & & & & & & & & & & & \\
\hline 562 & 0.1 & & 0.0 & & & & & & & & 1075.64 \\
\hline 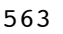 & & & & & & & & & & & \\
\hline 5 & & & & & & & & & & & \\
\hline & & & & & & & & & & & \\
\hline 66 & $10]$ & 704 & .0697 & .702 & 22 & & 0.397 & 1. & 5.669 & 67 & 1099.37 \\
\hline
\end{tabular}




$\begin{array}{lllllllllllll}567 & 0.101 & 4.5967 & 0.0699 & 0.701 & 0.41527 & -40.0 & 0.397 & 1.598 & 5.669 & 5.984 & 1105.34 \\ 568 & 0.101 & 4.6230 & 0.0701 & 0.699 & 0.41531 & -40.0 & 0.397 & 1.598 & 5.669 & 6.001 & 1111.33 \\ 569 & 0.101 & 4.6492 & 0.0703 & 0.697 & 0.41536 & -40.0 & 0.397 & 1.598 & 5.668 & 6.018 & 1117.34 \\ 570 & 0.101 & 4.6755 & 0.0705 & 0.695 & 0.41540 & -40.0 & 0.397 & 1.598 & 5.668 & 6.035 & 1123.36 \\ 571 & 0.101 & 4.7018 & 0.0707 & 0.693 & 0.41545 & -40.0 & 0.397 & 1.598 & 5.668 & 6.052 & 1129.40 \\ 572 & 0.101 & 4.7280 & 0.0709 & 0.691 & 0.41549 & -40.0 & 0.397 & 1.598 & 5.667 & 6.069 & 1135.46 \\ 573 & 0.101 & 4.7543 & 0.0710 & 0.690 & 0.41554 & -40.0 & 0.397 & 1.598 & 5.667 & 6.086 & 1141.54 \\ 574 & 0.101 & 4.7806 & 0.0712 & 0.688 & 0.41558 & -40.0 & 0.397 & 1.598 & 5.667 & 6.102 & 1147.63 \\ 575 & 0.101 & 4.8068 & 0.0714 & 0.686 & 0.41563 & -40.0 & 0.397 & 1.598 & 5.667 & 6.119 & 1153.74 \\ 576 & 0.101 & 4.8331 & 0.0716 & 0.685 & 0.41567 & -40.0 & 0.397 & 1.598 & 5.666 & 6.136 & 1159.86 \\ 577 & 0.101 & 4.8594 & 0.0718 & 0.683 & 0.41571 & -40.0 & 0.397 & 1.598 & 5.666 & 6.152 & 1166.00 \\ 578 & 0.101 & 4.8857 & 0.0720 & 0.681 & 0.41575 & -40.0 & 0.397 & 1.598 & 5.666 & 6.169 & 1172.16 \\ 579 & 0.101 & 4.9119 & 0.0722 & 0.679 & 0.41579 & -40.0 & 0.397 & 1.598 & 5.665 & 6.185 & 1178.34 \\ 580 & 0.101 & 4.9382 & 0.0724 & 0.677 & 0.41583 & -40.0 & 0.397 & 1.598 & 5.665 & 6.202 & 1184.53 \\ 581 & 0.101 & 4.9645 & 0.0726 & 0.675 & 0.41587 & -40.0 & 0.397 & 1.598 & 5.665 & 6.218 & 1190.74 \\ 582 & 0.101 & 4.9908 & 0.0728 & 0.674 & 0.41591 & -40.0 & 0.397 & 1.598 & 5.664 & 6.235 & 1196.96 \\ 583 & 0.101 & 5.0170 & 0.0730 & 0.672 & 0.41595 & -40.0 & 0.397 & 1.598 & 5.664 & 6.251 & 1203.20\end{array}$

